\title{
A SYSTEMATIC PROCEDURE FOR RESERVOIR CHARACTERIZATION
}

Annual Report for the Period

October 1, 1985-September 30, 1986

By

Larry w. Lake Gary A. Kocurek

Mark A. Miller
$\mathrm{DOE} / \mathrm{BC} / 10849--5$

DE8 8001206

December 1987

Prepared for

U.S. Department of Energy

Assistant Secretary for Fossil Energy

James W. Chism, Project Manager

Bartlesville Project Office

P.O. Box 1398

Bartlesville, OK $\mathbf{7 4 0 0 5}$

Prepared by

The University of Texas at Austin

The Department of Petroleum Engineering and

The Department of Geological Sciences Austin, Texas 78712 


\section{DISCLAIMER}

This report was prepared as an account of work sponsored by an agency of the United States Government. Neither the United States Government nor any agency Thereof, nor any of their employees, makes any warranty, express or implied, or assumes any legal liability or responsibility for the accuracy, completeness, or usefulness of any information, apparatus, product, or process disclosed, or represents that its use would not infringe privately owned rights. Reference herein to any specific commercial product, process, or service by trade name, trademark, manufacturer, or otherwise does not necessarily constitute or imply its endorsement, recommendation, or favoring by the United States Government or any agency thereof. The views and opinions of authors expressed herein do not necessarily state or reflect those of the United States Government or any agency thereof. 


\section{DISCLAIMER}

Portions of this document may be illegible in electronic image products. Images are produced from the best available original document. 


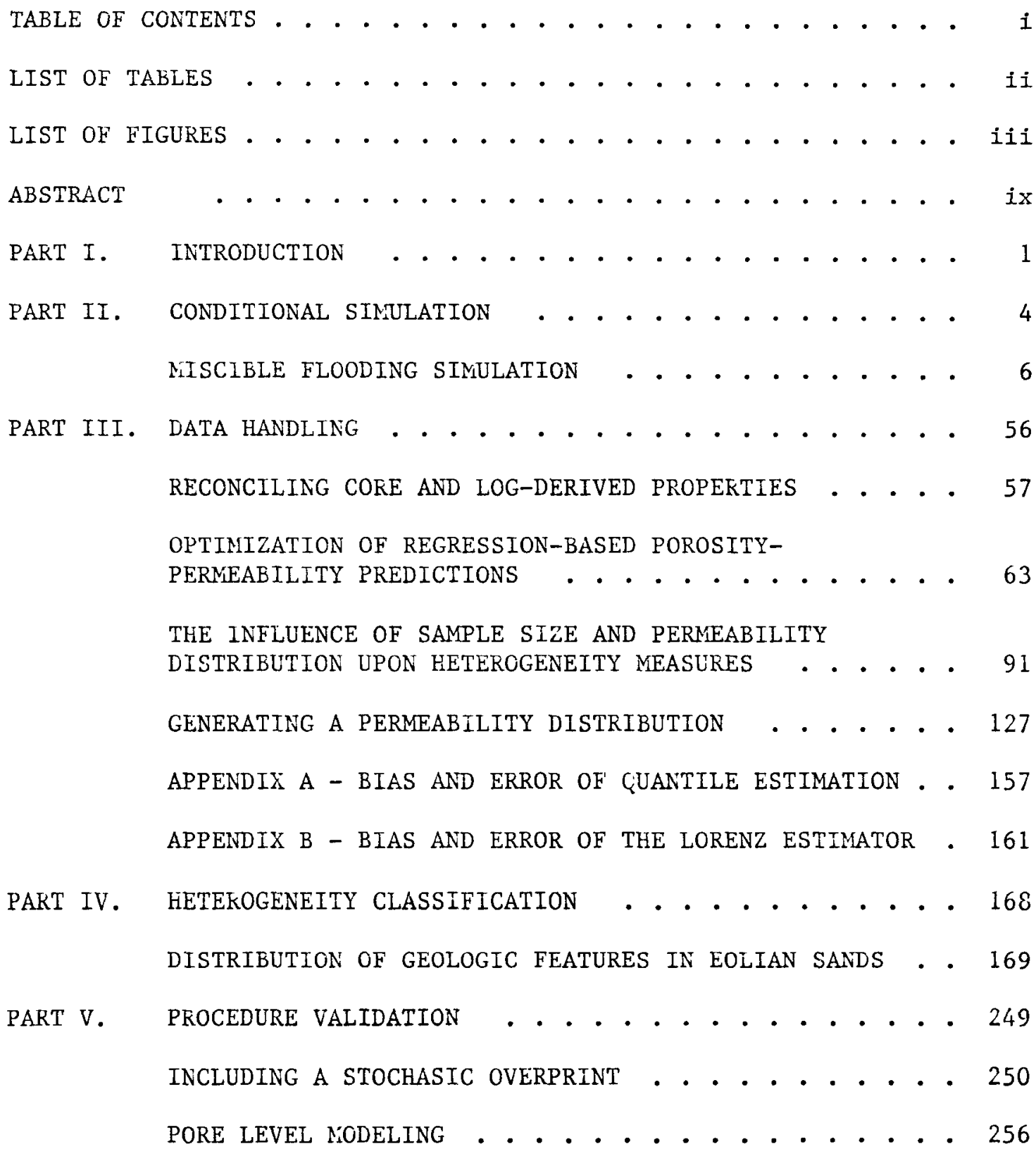


MARTE TIT-1. Nonte Car'o simulatior results for ?05 core plup "aluea. ................... s

TAFT.E III-2. Lionte rarlo simulation resultr for core hith $\geqq 28.85 . \ldots \ldots \ldots \ldots \ldots \ldots \ldots \ldots \ldots \ldots$

TARLE III-3. Nente Carlo simulation results for 295 core plug values. ............... 85

TART,F TII-L. Properties of data sets for reservoir simulations. ......................... $1 n^{n}$

Table IV-1. Rnunding surface and stratification lata for units of the A-complex. ............... 0 .

Table IY-2. Bourding surface ard stratification data for units of the R-comblex. ............... 210

Tále Tr-3. Nourding surfoce and stratification data for units of the C-complex. .............. ?11

Table rv-4. Rourding surface ard stratification data for units of the $\mathrm{E}$-compler. $\ldots \ldots \ldots \ldots \ldots \ldots \ldots \ldots .212$

Table IV-5. Rourdirg surface and stratification data for units of the E-complex. .............. 213

Table Ir-6. Dimensions of anme of the bedforms represented

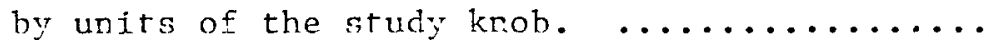




\begin{tabular}{|c|c|c|c|}
\hline igure & $I I-1$. & 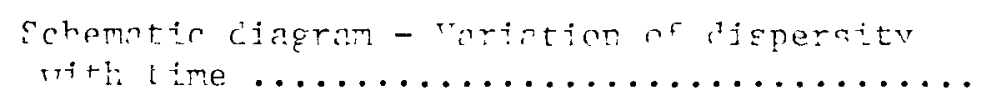 & - \\
\hline Fjgurs & $T^{++}=$ & 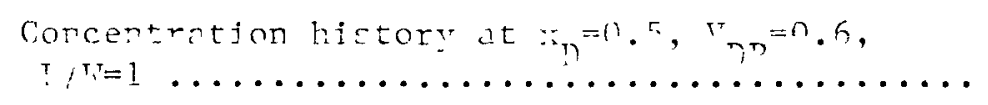 & 10 \\
\hline Firque & \pm-3 & 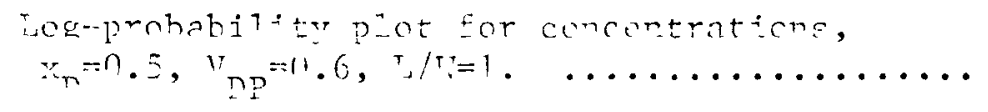 & 13 \\
\hline Eigure & $\therefore I-4$. & $\begin{array}{l}\text { Schematic travel patho of nartic }{ }^{\top} \text { es at a } \\
\text { cross-section. } \quad \ldots \ldots \ldots \ldots \ldots \ldots \ldots \ldots \ldots \ldots\end{array}$ & 14 \\
\hline Figure & $T T-5$ & $\begin{array}{l}\text { Lcg-probabslity plot corrected tom arrival } \\
\text { times, } \mathrm{x}_{\mathrm{D}}=0.5, \mathrm{~V}_{\mathrm{Dp}}=0.6, \mathrm{~T} / \mathrm{T}=1 . \quad \ldots \ldots \ldots \ldots\end{array}$ & 17 \\
\hline Tigure & I I - 6 & $\begin{array}{l}\text { Frrors in calculatipg macroscopic } \\
\text { dispersivities from } s ? \text { (re. } \quad \ldots \ldots \ldots \ldots \ldots \ldots\end{array}$ & 18 \\
\hline Figrure & II-? & 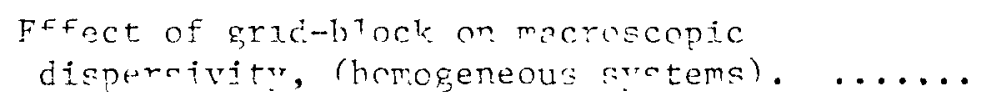 & 21 \\
\hline Figure & TI-B. & EEfect cf Dykstra-Parsons coefticient on $a_{M A} \ldots$ & 23 \\
\hline Figure & II-O. & $\begin{array}{l}\text { Nacroscopic dispersivities across the cross- } \\
\text { sections, } V_{n P}=0.6 \ldots \ldots \ldots \ldots \ldots \ldots \ldots \ldots \ldots \ldots \ldots\end{array}$ & 25 \\
\hline Figrure & IT -10 & 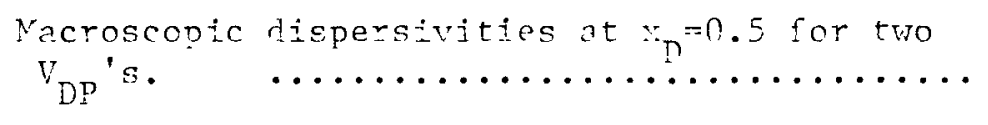 & 26 \\
\hline Tifure & $\underline{T}^{\top}-11$ & $\begin{array}{l}\text { Comparisor between mesascopic anc racroscopic } \\
\text { diapersinities } \quad \ldots \ldots \ldots \ldots \ldots \ldots \ldots \ldots \ldots \ldots \ldots\end{array}$ & 28 \\
\hline Figure & $\mathrm{IT}-12$ & $\begin{array}{l}\text { Fffect of correlation ength on macro/mega- } \\
\text { scopic dispersivities, }{ }_{\mathrm{SP}}=0.6, \mathrm{I} / \mathrm{T}=5 . \quad \ldots \ldots\end{array}$ & 30 \\
\hline Figure & II-13. & $\begin{array}{l}\text { Frfect of aspect ratio n macroscopic } \\
\text { dispersivities, }{ }_{\mathrm{DP}}=0.6 . \ldots \ldots \ldots \ldots \ldots \ldots\end{array}$ & 33 \\
\hline Figure & IJ -14 & $\begin{array}{l}\text { Macroscopic dispersivities at three cross- } \\
\text { sections, } V_{D P}=0.6, \mathrm{~L} / \mathrm{V}=5 . \quad \ldots \ldots \ldots \ldots \ldots \ldots\end{array}$ & 35 \\
\hline Figure & II-15. & $\begin{array}{l}\text { Macroscopic dispersivities at three cross- } \\
\text { sections, } V_{n \underline{p}}=0.6, \mathrm{~L} / \mathrm{H}=10 . \ldots \ldots \ldots \ldots \ldots \ldots\end{array}$ & 36 \\
\hline Figure & II-16. & $\begin{array}{l}\text { Stardard error in average macroccopic values, } \\
V_{\Gamma P}=0.6 . \cdots \cdots \cdots \cdots \cdots \cdots \cdots \cdots \cdots \cdots \cdots \cdots\end{array}$ & 38 \\
\hline Figure & $T^{-}-17$ & 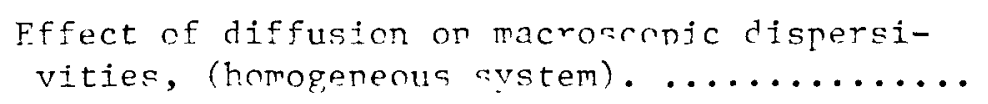 & $4 n$ \\
\hline
\end{tabular}


Tigure TJ-18. Thact or diffucion on racroscopir dionorsi-

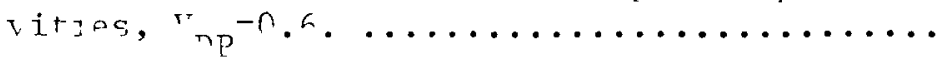

Tigure TT-1n. Ffect of diffucion nn macrescopic uianergi--

itiec at - croramsecticr, ${ }^{-}=0 .{ }^{5} . \ldots \ldots \ldots$

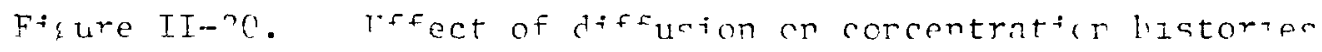

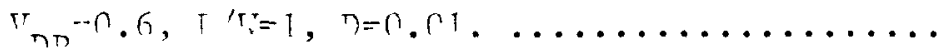

Digure Tr-'1.

Tract of diffucion on cementration hirtories,

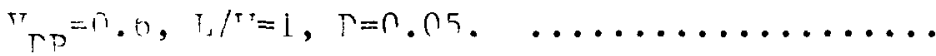

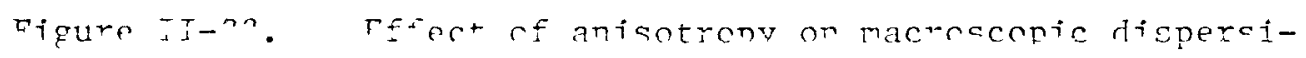

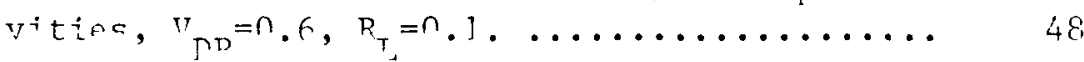

Figure TT-33. Iacroscopic diopersivitues at a croca-aection,

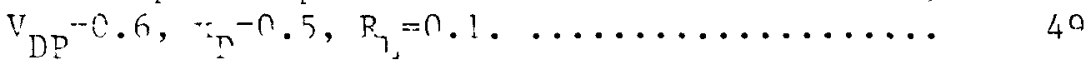

Figure TT-nt. Fifert of ditiotrong on macrosenpic dispergiritiea, $V_{\Gamma F}=0 . r, P_{T}=5.0 . \ldots \ldots \ldots \ldots \ldots \ldots \ldots$

Fioure IT-5. "acrosennic dicnereivities it a cronc-eretion,

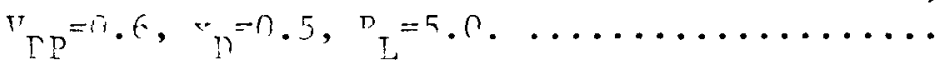

Figure ${ }^{T+} I_{-1}$. Decreared variance nbserver with increased ccato-rveraging measuremprt technique. ..... 50

Firurea ?'I-1a. Fypeted corre'ations ac $a$ functiol of acajeareraging czzp (any givon heterogrenejty).... 5n

Figure $\mathrm{I}^{\mathrm{T}} \mathrm{-n}^{-}$. Famili of curves depictjig corrotation for varicuc rcale-averaging techpiques for any

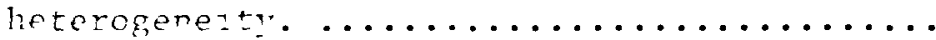

Figure TIIna. Trpectod correlaricra as a furction of heterogeneity (constant rcale-averagirg technique).

Figure TTI-3. Hamilr of curves depicting corrolation for various core sample densitjes as a function

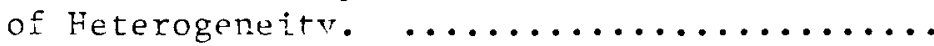

Figure IIT3a. Fxpected correlation as a function of sample densitr for any specific heterogeneity and specific scale-averaging technique.

Figure TTT-4. Hpothetical porosity-permeability plot. ....

Figure III-5. Porosity and permeability data for Fontainehleau sandstone (after. Tacquir, 1964). .....

Figure III-6. Fontaireblenu sandatone porosity hirtogram. .. 


\begin{tabular}{|c|c|c|c|}
\hline$\because \because$ mes & $T^{T}=-7$ & 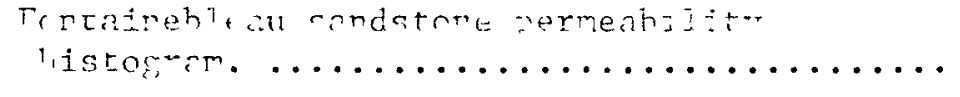 & 77 \\
\hline Ficure & ITT- & 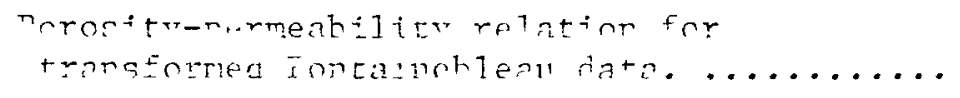 & 79 \\
\hline$=i_{g} u^{r} t$ & $3^{+\infty}-0$ & Portinebleau ceta and pred"ctive curves. . & $7 ?$ \\
\hline$m-s c r=$ & $T+T-1 n$ & 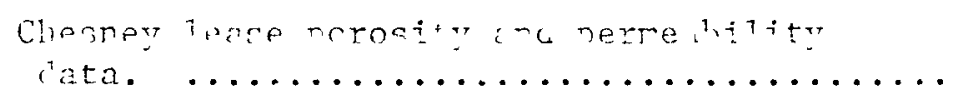 & $n$ \\
\hline$r$ ture & 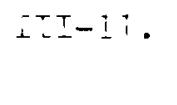 & 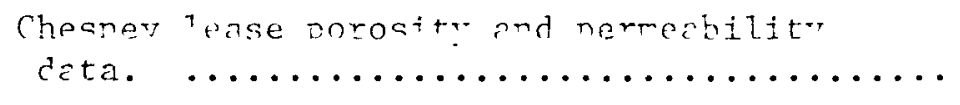 & 20 \\
\hline Figure & $I^{T^{\top}-}-i^{\prime}$ & 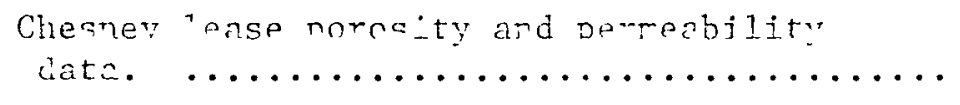 & 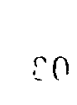 \\
\hline Figure & $\cdots-12$ & Checrey data and rrodictime curres. ......... & 83 \\
\hline Pigure & $I I I-{ }^{-4}$ & 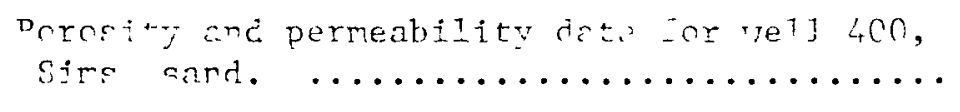 & $\{8$ \\
\hline Fistira & $-\underline{T}-15$ & Sima sanc data nd predictive curvece $\ldots$. & 3,8 \\
\hline Figure & ${ }^{\top \top} \mathrm{T}-16$ & $\begin{array}{l}\text { Sample size hehavior for the Dykstra-params } \\
\text { estimator. } \quad \cdots \cdots \cdots \cdots \cdots \cdots \cdots \cdots \cdots \cdots\end{array}$ & 96 \\
\hline Yigure & $=I^{-}-17$ & $\begin{array}{l}\text { Dias performance sor the roren: } \\
\text { estimator. } \quad \ldots \ldots \ldots \ldots \ldots \ldots \ldots \ldots \ldots \ldots \ldots \ldots\end{array}$ & 58 \\
\hline Figure & $T^{T} \pm-18$ & $\begin{aligned} \text { Sample size belarior for the torenz } \\
\text { estimator. } \\
\quad \ldots \ldots \ldots \ldots \ldots \ldots \ldots \ldots \ldots \ldots\end{aligned}$ & 100 \\
\hline Tigure & $I^{\top}-1 S$. & $\begin{array}{l}\text { Jorenz coefficients for populations having } \\
\text { 1, ccn-md medior permeability. } \quad \ldots \ldots \ldots \ldots \ldots\end{array}$ & 105 \\
\hline Figure & TTI-20. & Hopothetical Wretra-Parsons plot for $p>n \ldots$ & $10^{7}$ \\
\hline Figure & III-? & $\begin{array}{l}\text { Log-normal probability plot for data } \\
\text { Set No. } 5 . \ldots \ldots \ldots \ldots \ldots \ldots \ldots \ldots \ldots \ldots \ldots\end{array}$ & 111 \\
\hline Fisure & III-2?. & 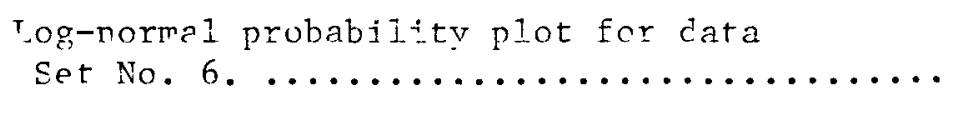 & 112 \\
\hline Iigure & III-23. & $\begin{array}{l}\text { Log-normal probability plot for data } \\
\text { Set No. } 6 . \cdots \ldots \ldots \ldots \ldots \ldots \ldots \ldots \ldots \ldots \ldots \ldots \ldots \ldots\end{array}$ & 113 \\
\hline Figure & III-?4. & $\begin{array}{l}\text { Log-normal probability plot for data } \\
\text { Set No. } 9 . \ldots \ldots \ldots \ldots \ldots \ldots \ldots \ldots \ldots \ldots \ldots \ldots \ldots \ldots\end{array}$ & 114 \\
\hline Ficlire & III-25. & 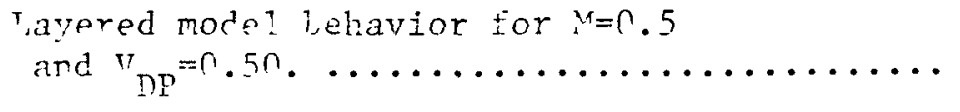 & 116 \\
\hline
\end{tabular}


Figure III-26. Luyered model behavior for $M=2.0$

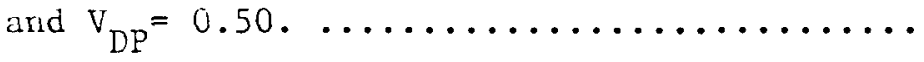

Figure III-27. Layere 1 model behavior for $Y^{\prime}=0.5$

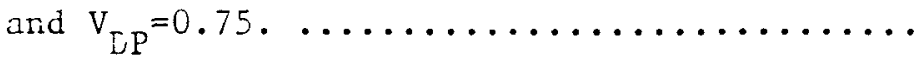

118

Figure III-28. Layered model behavior for $M=2.0$

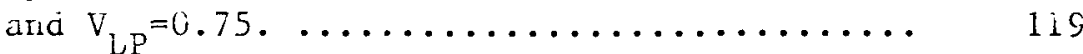

Higure III-29. Felative performance of heterogeneity

measures for a iayered rode1........... 125

Figure 1 II-30. Standard deviation of the mean. ......... 133

Figure IIF-31. Constructed autocorrelation for sample

span/correlation length $=5$. .............

Figure III-32. Constructed autocorrelation for sample

spari/correlation length $=1 . \ldots \ldots \ldots \ldots$

Figure III-33. The variability of iine processes. ........ 139

Figure III-34. The variability of the covariogram. ....... 140

Figure III-35. Uncertainty of the variogran. .......... 145

Figure III-36. A comparison between the correlogram and

the variogram. $\ldots \ldots \ldots \ldots \ldots \ldots \ldots \ldots \ldots$

Figure III-37. Effect of correlarion on the cumulative

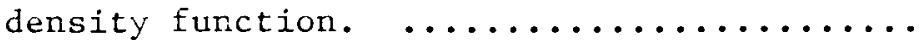

Figure III-38. Effects of p-normal transformation on

variogram. $\ldots \ldots \ldots \ldots \ldots \ldots \ldots \ldots \ldots \ldots \ldots \ldots \ldots \ldots \ldots$

Figure III-39. Effects of transform of probability

density function. ................ 155

Figure IV-1. Subhorizonatal, first-order bounding surfaces separating cross-bedded units of the

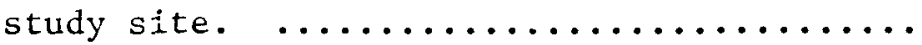

Figure IV-2. Grailiflow and wird-ripple deposits on a small (approx. 6 II) slipface, Algodones dune field, Caiifornia.

Figure IV-3. Grainflow stratification in the Page sandstone at the study site. $\ldots \ldots \ldots \ldots \ldots \ldots \ldots \ldots$.

Figure iV-4. Wind-ripple stratification in the Page sandstore at the study site. ............... 


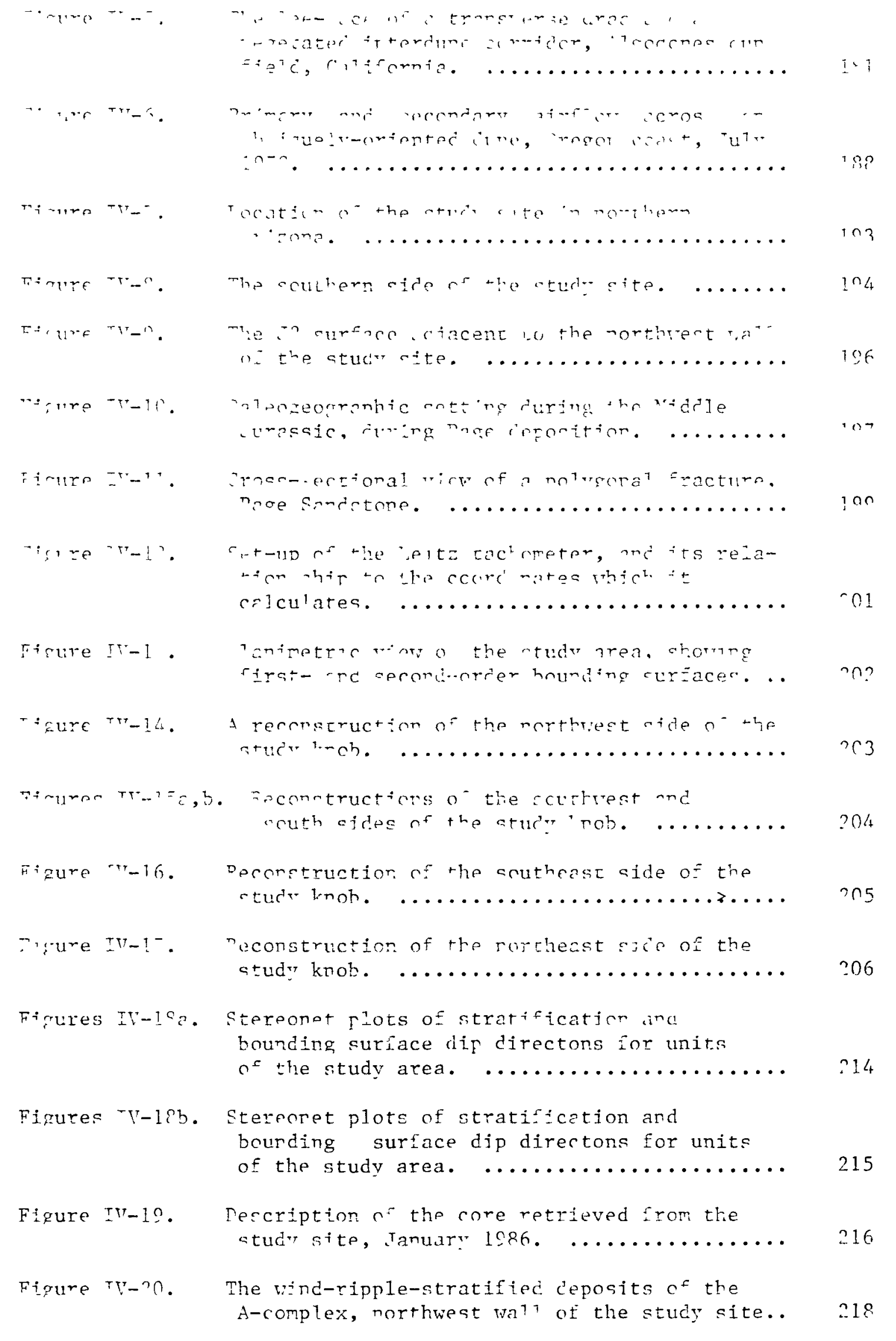




\begin{tabular}{|c|c|c|}
\hline Eioure & $=1-21$ & 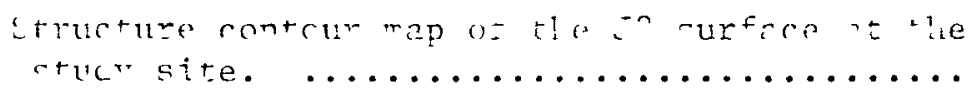 \\
\hline$\overline{4}$ irure & rYT... 2 . & 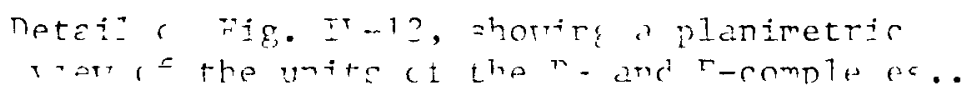 \\
\hline Ti zure & TY- & 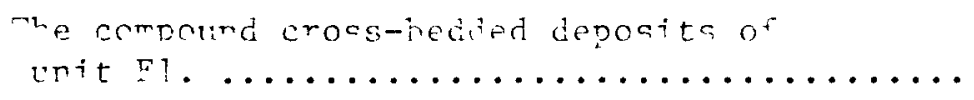 \\
\hline Figure & Ir...?'t. & $\begin{array}{l}\text { chematic cross-section and interpretation } \\
\text { cf the crosabecced secunnce of the nage } \\
\text { sancrtore at the study cite. } \ldots \ldots \ldots \ldots \ldots \ldots\end{array}$ \\
\hline Eiguro & $\mathrm{T}-25$ & 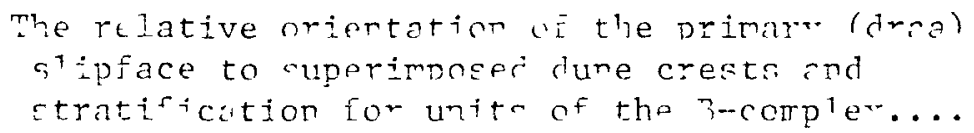 \\
\hline Figure & II-7h. & 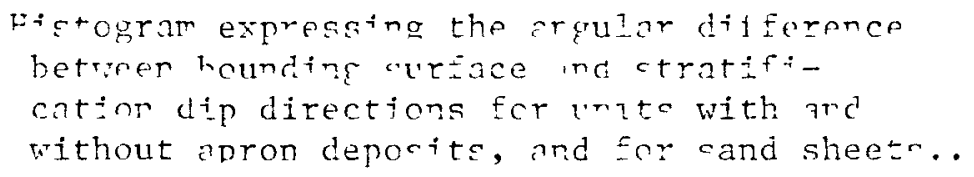 \\
\hline rigure & $r-1$. & remparicor a' input ctic comoratec rariograms.. \\
\hline Figure & $\mathrm{V}-2$ & $\begin{array}{l}\text { Comparison of input and zererated } r \text { pectral } \\
\text { Density Furctions. }\end{array}$ \\
\hline Figure & 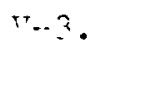 & $\begin{array}{l}\text { Ceneratoc and input cumulatire probability } \\
\text { dercity Eunctior } . \ldots \ldots \ldots\end{array}$ \\
\hline Figure & $r-4$. & 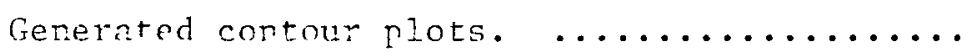 \\
\hline
\end{tabular}


This report, a jear's progress on "is Systemtatic Proceuture for Fitservoir Characterication", deals with a variety or topics ali centered around the main goal of naking numerlcal reservoir simulation results conform nore closely with geologic descriptions.

The first part of the report discusies results on conditional simulations of miscible displacements in randomly heterogeneous permeable media. The focus here is on local or macroscopic dispersich, the dispersion experienced at a fixed point in the medium. Macroscopic dispersivity has many of the same dependencies on reservoir properties as does megascopic dispersivity, but it seems to be less time dependent and is always smalier. We have iot discovered a mathematical model to describe its behavicr.

A najor fortion oj the report deais with statistica descriptions. We investigate the bias and precision of standard measures of heterogeneity, the Lorenz ard Dykstra-Pursons coefficient. After this, the work explores the benefits of using a distribution type characterization parameter in exploring heterogeneity. We find that distribution type is as sensitive as mobility ratio $i_{i}$ determinins sweep efficiency. Other statistical topics dealt with include addressing the sampling issue in correlating log-cierived and core permeatilities and in generating a two-dimensional stochastic field that mimics an actual eolian reservoir.

The final major portion of the report describes our mapping efforts on the Page sandstone outcrop in northern Arizona. The mapping is to be used in generating both deterwinistic descriptions and in calibrating the stochastic descriptions discussed above. 
A SYSTEMATIC PROCEDURE FOR RESERVOIR CHARACTERIZATION

Annual Report October 1, 1985 - September 30, 1986

PART I. INTRODUCTION

We define reservoir characterization as the process of assigning spatially varying input to a numerical simulator. This input should reflect the geologic classjications existing at the point to be estimated and contain the correct deterministic trends if such exist. The other components will be captured from statistical descriptions (most likely values, variations, and spatial correlations) for each geologically distinct unit. Finally, the input should be adjusted for the scale of the minimum granularity (grid size) of the simulation.

A possible procedure for reservoir characterization is:

1. Identify and map geologic units present in the reservoir. Unit boundaries may be observed from well-to-well correlations, assigned from a geologic model, or taken as stochastic variables themselves.

2. For each geologic unit gather and process data to isolate trends and to determine the statistical descriptions. Hydrologically homogeneous units may then be identified. These may or may not coincide with the geologic units. Clearly, this step will place considerable reliance on outcrop and wellbore (core/log) characterizations.

3. Cover the region of interest in the reservoir with an appropriate grid. Each grid falling within a given hydrologic unit is assigned properties based on the deterministic trends and statistics. Grids falling on top of unit boundaries require special attention, 
inasmuch as the nature of the boundary itself (for example, shale or erosional surface) will determine parameter assignments.

4. Values assigred in item 3 must be adjusted for the size of the grid blocks, since blocks are invariably much larger than the scale of variabilty. For deterministic trends, this involves assigning "pseudo" properties; for the other variations we will adjust dispersivities, capillary pressures, and/or fingering factors.

5. Each ensemble of parameters must be conditioned to agree with aggregate measures, such as well tests, or with actual measurement if a grid block happens to contain a well.

The work discussed here can not fully develop this entire plan in detail. A thorough methodology requires a degree of validation (comparison between actual and predicted results) which is beyond the scope of the original work (Lake et al., 1985). In the following report we propose several steps which are intended to investigate the validity of the proposed procedure and to expose the strengths and weaknesses.

This work falls into four parts. Under the heading of "Conditional Simulations" we discuss results of simulations applied to stochastic fields. In the area of "Improved Data Handling" we give results of reconciling core and log data using non-normal transformations for improved regression, bias of heterogeneity measures, and methods to generate stochastic fields. Under "Heterogeneity Classification" we discuss the distribution of eolian geologic features and relate these to statistical measures. "Procedure Validation" concludes by giving results of generating 
two-dimensional stochastic fields and the results of pore level modeling.

PART I. REFERENCES

Lake, L. W., M. A. Miller, and G. A. Kocurek, Department of Energy Proposal, 1985. 
PART II. CONDITT ONAL SIMULATINN

Conditional simulation is a stochastic approach to an indeterminate problem. Oil reservoirs are invariably heterogeneous. Normally the only information available is the measurements at certain points through the wells. As to the properties between these points, assuming the measurements at these points are reliable, the best we can do is to interpolate using statistical information: spatial correlation and variation according to the geological environment. Both topics are covered in later parts of this report.

When a field is to be simulated, at best the following information is available:

1. Deterministic properties at certain given points,

2. Spatial distribution or correlation of the properties.

A conventional simulation can use only the deterministic part of the information; therefore, there is a lot of uncertainty in the scaling and averaging of permeability values. In a conditional simulation a stochastic field is generated using the given correlation function, which may be obtained from geological characterization, and then "conditioned" to be consistent with the deterministic information. A simulation run using this conditioned stochastic field generates a possible outcome. A large number of such simulation runs with independent stochastic fields will then give a statistical view of the problem.

This approach not only gives a more realistic result by including heterogeneity with both deterministic properties, physics and 
indeterministic information, but it also can give an estimate of the possible error due to the uncertainty of the given information.

Another application of the method is to solve the problem of scaling. Because of the limit on the number of grid blocks in a computer simulation, details inside the blocks will be averaged out. Conditional simulations run at different scales can give an effective scheme for averaging the properties. 
MISCIBLE FLOODING SIMULATION

The conditional simulation discussed in this section consists of first-cortact miscibie, equal viscosity, equal density displacements in randomly heterogeneous permeable media. The media are random in permeability which are distributed according to Heller's method with preconditioned amount of variability (as measured through a correlation leigth). See Part III for theoretical treatments of both statistical measures of variability and correlation. See also Arya (1986) for details on the Helier procedure and its properties.

Earlier work (Arya et a1., 1986) has established the behavior of cross- sectionally averaged (megascopic) dispersivities in randomly heterogeneous media. To summarize briefly, $\alpha_{\mathrm{ME}}$ generally varies with dimensionless time as shown in Fig. II-1. For smali $t_{D}$, $\alpha_{\mathrm{ME}}$ grows lineariy with $t_{D}$ indicating channelling; for large $t_{D}, \alpha_{M E}$ is constant indicating pureiy dispersive behavior. The transition between channelling and dispersive behavior is smooth and occurs over a finite time interval. During the channeling mixing zones grow in proportion to $t_{D}$; during dispersive mixing the zones grow with the square root of ${ }^{t}$ but they do not lose the features of the initial channeling behavior.

Based on the seminal work of Taylor (Arya, 1986) the megascopic dispersivity varies according to

$$
\frac{\alpha_{M E}}{L}=\frac{C_{v}^{2}}{t_{D}}\left[\lambda_{D} t_{D}-\lambda_{D}^{2}\left(1-e^{-t_{D} / \lambda_{D}}\right)\right]
$$

for randomly heterogeneous media with exponential correlation. $\mathrm{C}_{\mathrm{v}}$ is the coefficient of variation and $\lambda_{D}$ is the dimensionless correlation 


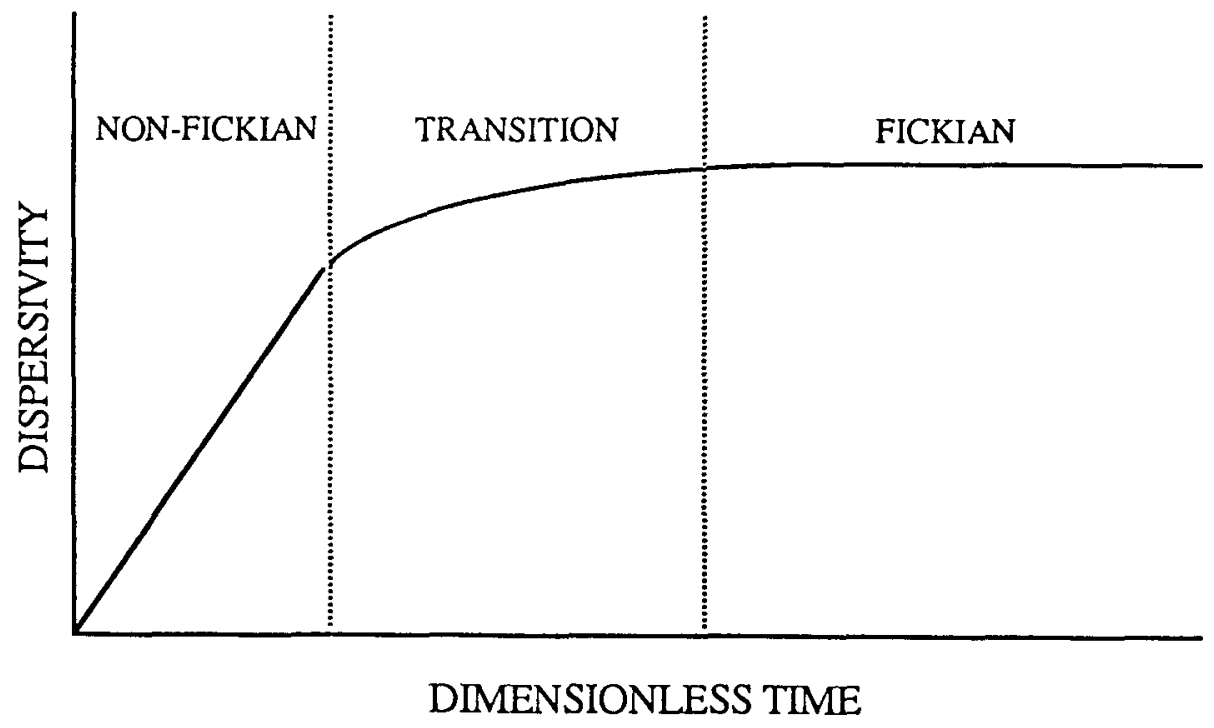

Figure II-1. Schematic diagram - variation of dispersity with time. 
length of the permeability field. The behavior of the above equation reproduces that in Fig. II-1. Two special cases are of interest. If the correlation length is much smaller than the medium length $\lambda_{D} \ll$ 1) $\alpha_{\mathrm{ME}}$ becomes

$$
\frac{\alpha}{\mathrm{ME}}=\mathrm{C}_{\mathrm{v}}^{2} \lambda_{\mathrm{D}}
$$

where the mixing is dispersive. If the correlation length is large we we have

$$
\frac{\alpha_{M E}}{L}=\frac{C_{v}^{2}}{2} t_{D}
$$

where the mixing is through channeling and the medium is effectively layered. If $\lambda_{D}$ is infinite, the medium is strictly layered. Both cases pertain during certain types of permeable media flow.

In the current section we continue this work through simulations from which we will derive the macroscopic dispersivity $\alpha_{\mathrm{MA}}$.

Macroscopic Dispersion in Permeable Medium - Macroscopic dispersivity is the point value of dispersivity obtained at any $(x, y)$ location in the permeable medium. The macroscopic dispersivity, $\alpha_{M A}$ controls the rate of mixing and thus the formation of a miscible front in solvent flooding. The variables which affect megascopic dispersivity, such as the magnitude of heterogeneity (Dykstra-Parsons coefficient ${ }_{D P}$ ), the spatial correlation for permeability), the aspect ratio of the two-dimensional model, and the magnitude of molecular diffusion also influence the macroscopic dispersivity though by a different magnitude and also in some cases in a different manner than for $\alpha_{M E}$. 
Procedure for Calculating Macroscopic Dispersivity - In this section the method used to calculate macroscopic dispersivity at any point in the two-dimensional, finite-difference computer simulations is outlined. Macroscopic dispersivity is a measure of the local mixing in the permeable medium. It can be measured at any point in the two-dimensional domain. In a numerical mode1 the dispersivity measured at a grid-block is considered to be macroscopic. Macroscopic dispersivity $\alpha_{M A}$ is primarily a function of the local heterogeneity but is influenced by the variations in transport properties of the entire system under consideration.

In order to determine the macroscopic dispersivity at any location in the numerical model, the concentration history at that location is required. By "concentration history" we mean the variation In concentration at any location as a function of dimensionless time $\left(t_{D}\right)$ or injected pore volumes. The arrival of a specific concentration contour at any location depends on a variety of factors such as the degree of heterogeneity and the distribution of permeabilities. Thus, in a heterogeneous permeable medium, at any fixed $x$-location, the fluid arrives at different times across the cross-section. This is because of variations in permeabilities being transformed into variations in fluxes, and therefore, the fluid travels at different velocities in different regions. This variation in travel velocities is seen in Fig. II-2, which shows concentration histories at $x_{D}=0.5$ for a simulation case. In this case, the $V_{D P}$ is 0.6 and the aspect ratio of $(\mathrm{L} / \mathrm{W})$ the system is one. There are 40 grid blocks in the $y$ direction and therefore we have forty different concentration histories. When the permeable medium is homogeneous, the velocity 


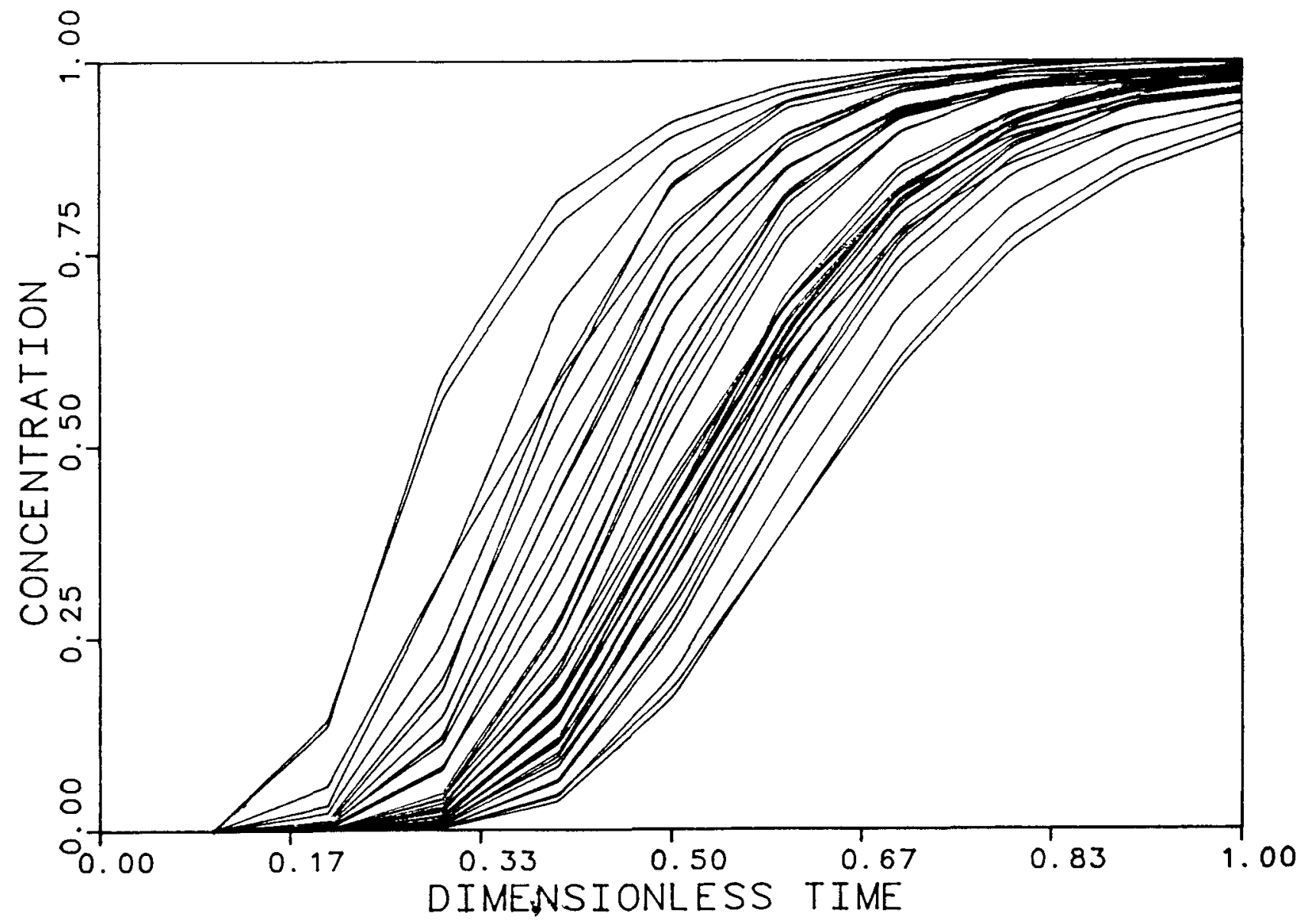

Figure II-2. Concentration history at $\mathrm{x}_{\mathrm{D}}=0.5, \mathrm{~V}_{\mathrm{DP}}=0.6, \mathrm{~L} / \mathrm{W}=1$ 
gradients are also uniform. In such a case, the fluid travels uniformly across every cross-section and at any fixed $x$-location (i.e., location in the flow direction), the concentration is the same across the cross-section. Therefore, all history curves are identical. Thus, the width of the band over which the concentration histories are distributed gives some idea about the magnitude of heterogeneity. A large distribution of concentrations at any cross-section also means a displacement with channeling. Large transverse mixing will result in a narrow band of concentration histories.

This concentration history at any grid block can be matched with an analytical solution of the one-dimensional convective-diffusion (C-D) equation to determine the macroscopic dispersivity in that grid block. An example of such an analytical solution would be the solution for the infinite boundary conditions which can be rewritten as

$$
C_{D}=\frac{1}{2}\left(1-\operatorname{erf}\left(\frac{x_{D}-t_{D}}{2 \sqrt{t_{D} / N_{P e}}}\right)\right)
$$

Rearranging this equation,

$$
\frac{x_{D}-t_{D}}{2 \sqrt{t_{D} / N_{P e}}}=\operatorname{erf}^{-1}\left(1-2 C_{D}\right)
$$

The above expression can be written in the form of the equation of a straight line

$$
\frac{x_{D}-t_{D}}{\sqrt{t_{D}}}=2 \sqrt{N_{P e}} \operatorname{erf}^{-1}\left(1-2 C_{D}\right)
$$

A plot with erf ${ }^{-1}\left(1-2 C_{D}\right)$ on the x-axis and $\left(x_{D}-t_{D}\right) / \sqrt{t_{d}}$ on the y-axis is a straight line for a fixed value of $x_{D}$. This plot is also known as the log-probability plot because plotting concentration (instead of 
the inverse error function) on a log-probability paper gives the same figure. The macroscopic dispersivity can be calculated from the slope which is equal to $2 \sqrt{\mathrm{N}} \mathrm{Pe}$. This procedure can be repeated for every point in the two-dimensional flow domain. The macroscopic dispersivity calculated using the above procedure is only an approximate value since the solution of the $C-D$ equation with boundaries at infinite distance is used. There is ar additional approximation because a 1-D solution is used in a 2-D field.

Figure II-3 shows such a log-probability plot at a fixed $x$ location (at $\mathrm{x}_{\mathrm{D}}=0.5$ ) for every point in the cross section. Each point has a unique value of slope which depends on the concentration history at that point. In this specific case, $V_{D P}$ is 0.6 and Fig. II-3 is derived from Fig. II-2 which shows the corresponding concentration histories. This matching procedure is different from the one used to determine megascopic dispersivities. In the case of megascopic dispersivities concentration profiles $\left(C_{D}\right.$ vs. $\left.x_{D}\right)$ averaged across a cross-section are used to determine dispersivities. But to calculate macroscopic dispersivities concentration histories $\left(C_{D}\right.$ vs. $\left.t_{D}\right)$ at every grid-block are used. Thus, the megascopic dispersivity $\alpha_{\mathrm{ME}}$ is determined as a function of time and the macroscopic dispersivity $\alpha_{M A}$ is determined as a function of spatial location $\left(x_{D}, y_{D}\right)$.

In a heterogeneous system, the arrival time for concentration at each block will depend on the path taken by the particle to traverse from the inlet to its present location. The shortest path is a straight line, but due to the tortuous nature of the permeable medium, the path taken by each particle is different. Figure II-4 shows the 


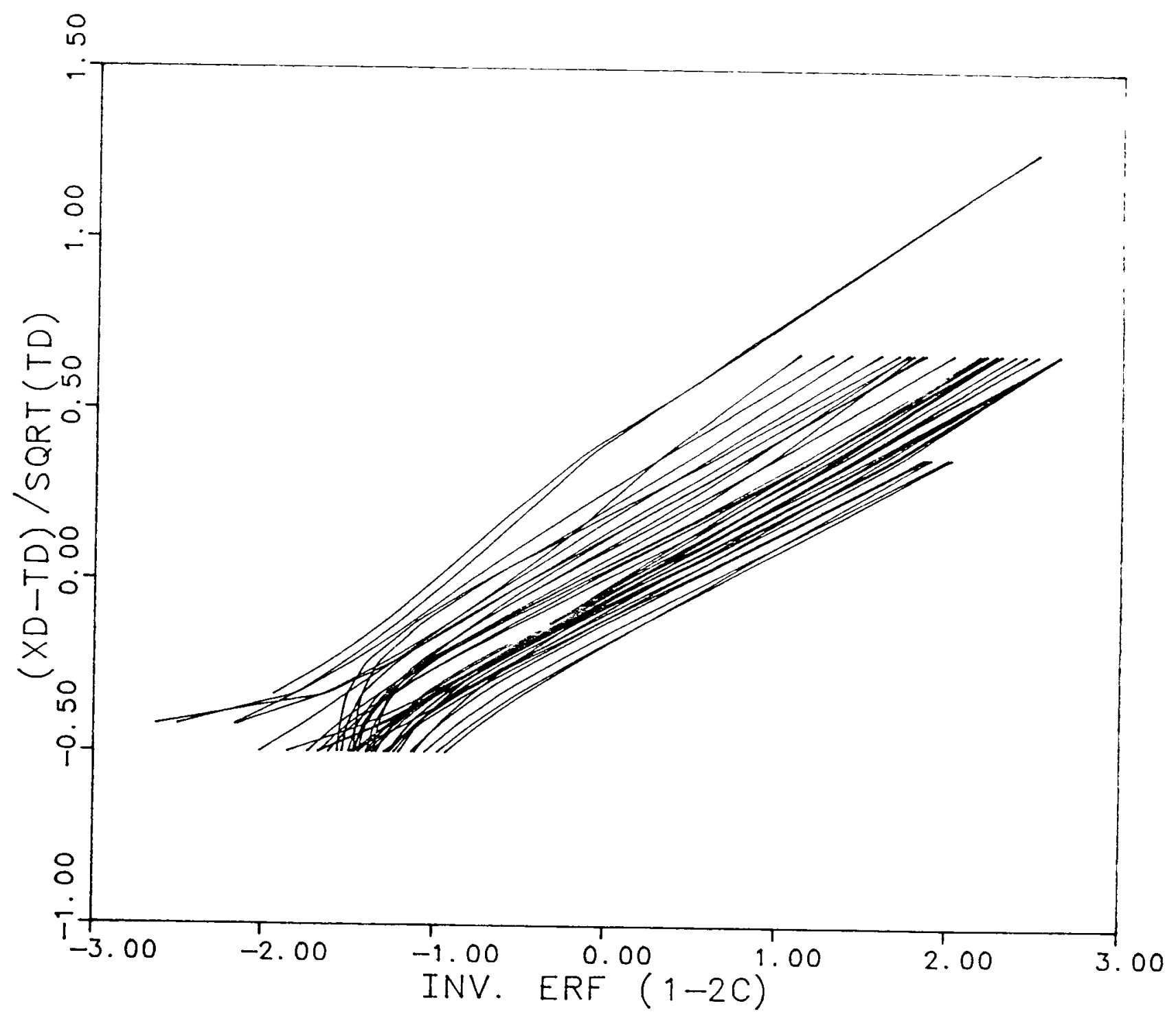

Figure II-3. Log-probability plot for concentrations, $x_{D}=0.5, v_{D P}=0.6, L / \mathrm{w}=1$. 

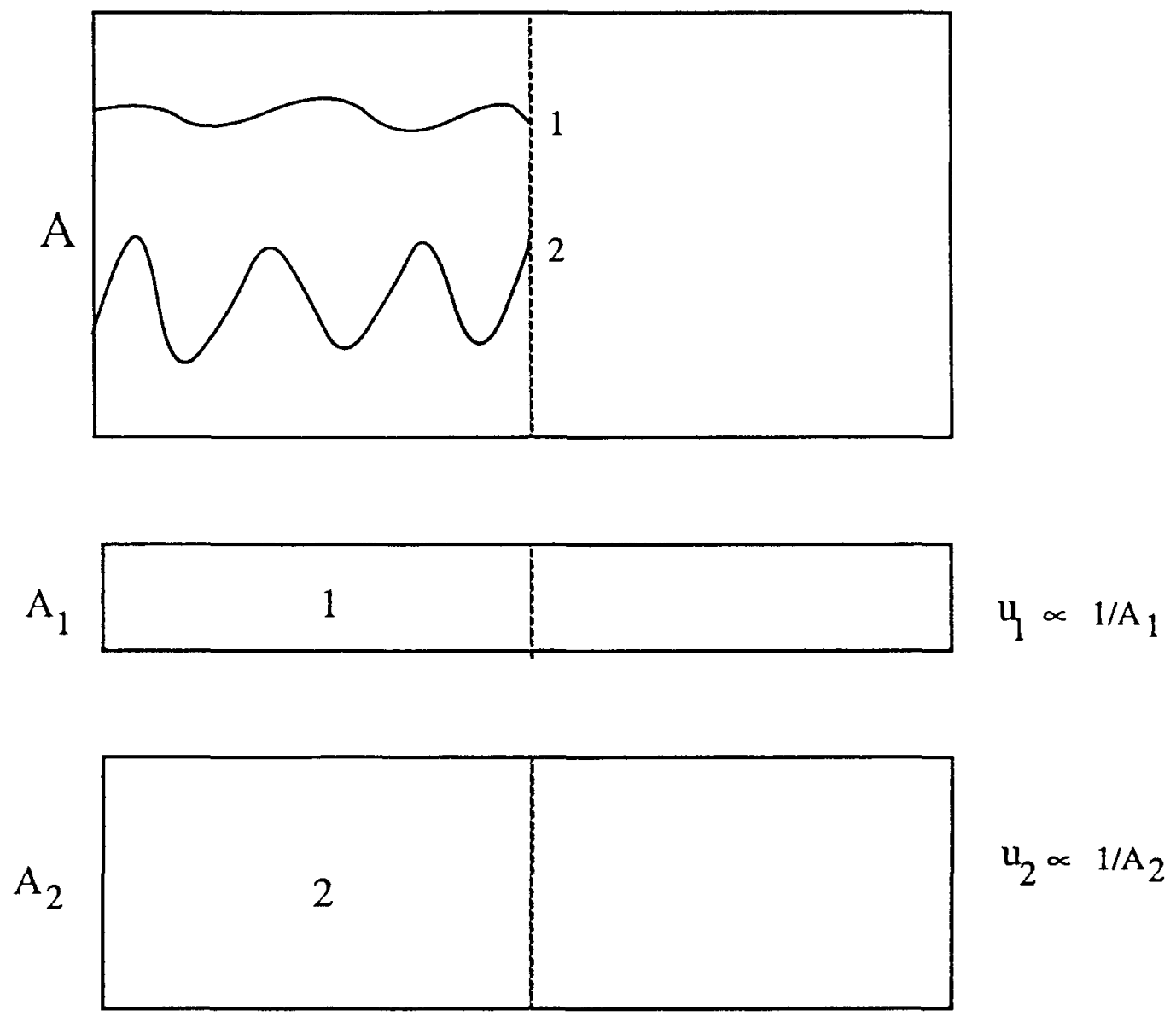

Figure II-4. Schematic travel paths of particles at a cross-section. 
schematic diagram for a hypothetical situation, when two paricles take completely different paths to arrive at the fixed $x_{D}$ location. The equivalent area travelled by each particle depends on its path and the velocity of each is inversely proportional to the area covered. The breakthrough time for any particle is given as

$$
t^{B T}=x / u
$$

And that for farticle " $1 "$ "

$$
t_{1}^{\mathrm{ET}}=\mathrm{x} / \mathrm{u}_{1}
$$

From the above equations, $u_{1}$ can be expressed as

$$
u_{1}=\frac{t^{B T}}{t_{1}^{B T}}
$$

Therefore from Eq. (II-2),

$$
u_{1} \frac{t}{L}=u \frac{t^{B T}}{t_{1}^{B T}} \frac{t}{L} \frac{t_{D}^{B T}}{t_{D_{1}}^{B T}} t_{D}=\text { where } t_{D}=\frac{u t}{L}
$$

However, in an ideal displacement, $t_{D}^{B T}=x_{D}$, and therefore

$$
u_{1} \frac{t}{L}=x_{D} \frac{t_{D}}{\tau_{D_{1}}^{B T}}
$$

Or in general,

$$
t_{D_{i}}=u_{i} \frac{t}{L}=x_{D} \frac{t_{D}}{t_{D_{i}}^{B T}}
$$


The above definition for $t_{D i}$ can be used in place of $t_{D}$ in Eq. (II-1) (or Eq. II-2) to correct for different arrival times for concentrations at each point across the cross-section at a fixed $x$ location. This correction results in the arrival of $C_{D}=0.5$ for all points across the cross-section at any location $\mathrm{x}_{\mathrm{D}}$ such that $t_{\mathrm{D}}=\mathrm{x}_{\mathrm{D}}$. Figure II-5 shows such a corrected plot for the results in Fig. II-3. The slope of each corrected line is then determined and the macroscopic dispersivity is calculated using Eq. (II-1). All the 1ines in Fig. II-5 are not perfectly straight because of the error associated with the analytical solution which is not exact (Brigham, 1974). The slope of each curve should be taken in the portion which is nearly straight and has the best fit (i.e., the highest linear regression coefficient). It was observed that taking the slope at $(0,0)$ (where $x_{D}=t_{D}$ and $C_{D}=0.5$ ), was a very good approximation in most of the cases. However, if the point of observation is located in a very high permeability block, the concentration may reach the maximum very rapidly and in such a case, the slope of the straight line at $(0,0)$ is very high. Figure II-6 shows such a situation. In this case, the true macroscopic dispersivity is given by the straight line portion of the curve and not by slope at $(0,0)$. Thus, care must be exercised to account for these variations when macroscopic dispersivities are calculated at each point. The dispersivities calculated at any fixed $x_{D}$ location (there are $N_{y}$ values) are then averaged to determine the mean macroscopic dispersivity at that $x_{D}$ location.

It may not be possible to determine dispersivities for every point in the cross-section due to one of several reasons. It is 


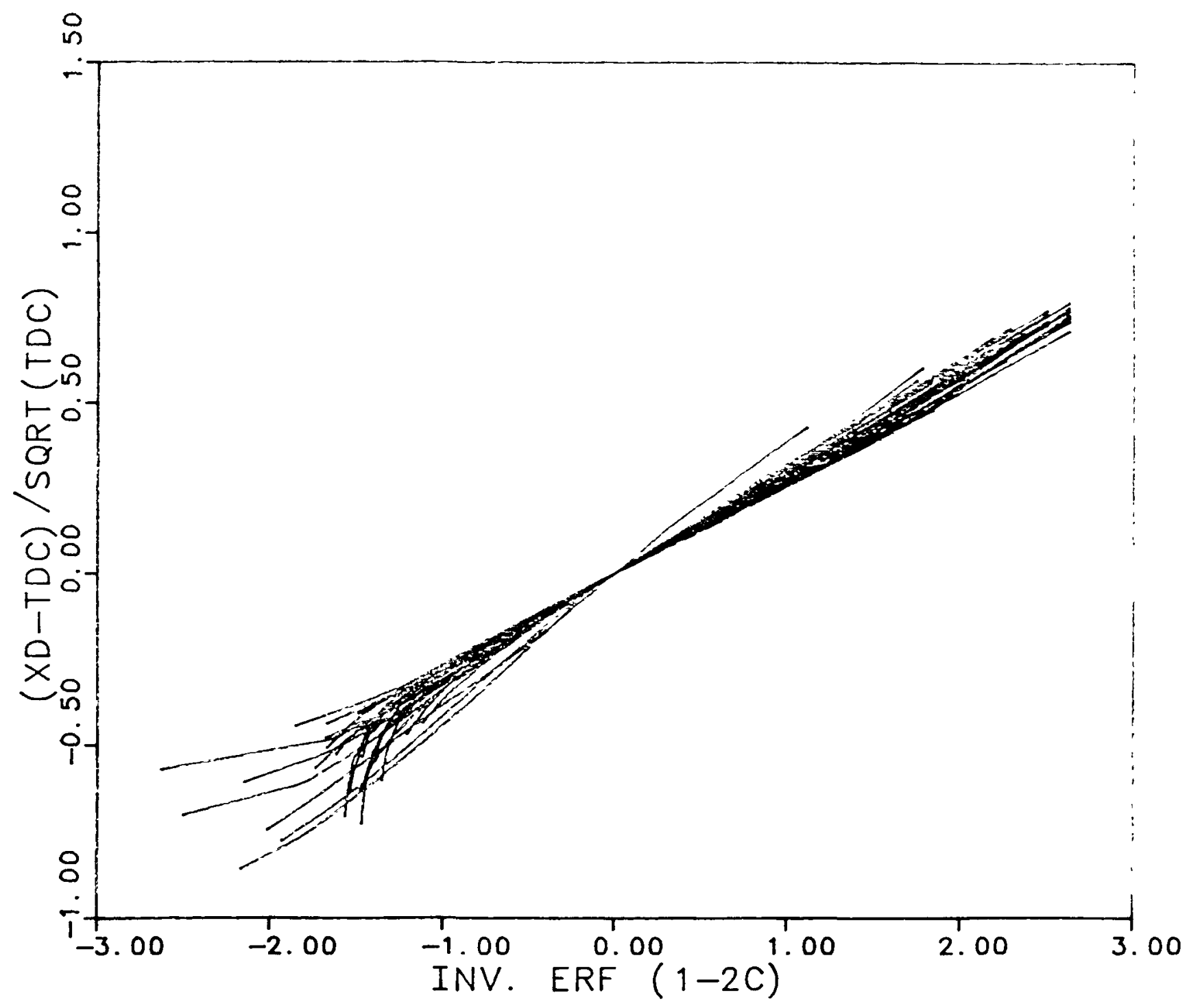

Figure II-5. Log-probability plot corrected for arrival times, $\mathrm{x}_{\mathrm{D}}=0.5, \mathrm{~V}_{\mathrm{DP}}=0.6, \mathrm{~L} / \mathrm{W}=1$ 


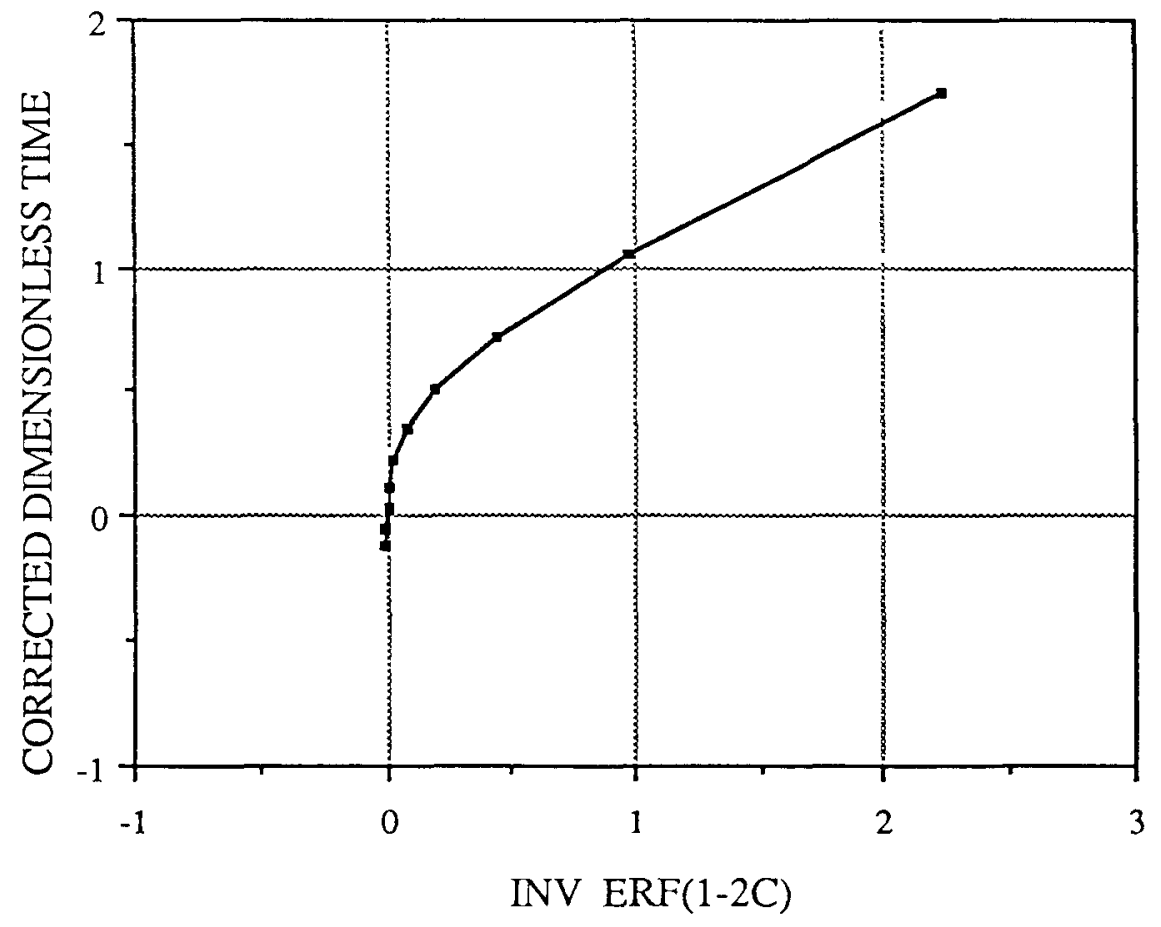

Figure II-6. Errors in calculating macroscopic dispersivities from slope. 
possible that all the concentrations at a grid-block are either below or above the $(0,0)$ point in the log-probability plot for concentration. In such a case that point cannot be used for measuring dispersivity because the $(0,0)$ point is needed to correct for the different arrival times. This situation typically arises at points very near or very far from the injection face where concentration changes occur either very rapidly or very slowly. It can also happen in regions with very high or very low permeabilities. This results in a different number of total points (across a cross-section) along the $\mathrm{x}$ locations where macroscopic dispersivities are averaged. The average $a_{M A}$ values are influenced by the total number of macroscopic dispersivities used to determine the average. This effect is corrected by calculating the standard error for the mean $\alpha_{M A}$ at each location. This standard error calculation takes into account the number of points used to determine the average $\alpha_{M A}$. In addition to the average $\alpha_{\text {MA }}$ values, the curves indicating the high (averagetstandard error) and low (average-standard error) values for dispersivities are also shown. The high and low values indicate the degree of variation in $\alpha_{M A}$ 's at that location. The standard error is calculated at the 95 percent confidence leve1 and is $\alpha_{M A} \pm 1.96 \sigma / \sqrt{n}$ where $\sigma$ is the standard deviation of $\alpha_{M A}$ and $n$ is the number of points in the set.

Macroscopic Dispersivity in a Homogeneous Medium - There are two reasons for calculating macroscopic dispersivities in a homogeneous system. First, to determine the accuracy of the procedure described in the previous section for determining $\alpha_{M A}$. Second, to determine if the correction procedure used for correcting megascopic dispersivities for 
numerical dispersion is also applicable for macroscopic dispersivities.

In a homogeneous medium there is no physical dispersion and the displacement is stable. The concentration front moves uniformly with the same velocity at every point across a cross-section and the macroscopic dispersivity is the same at every point across any cross-section. This dispersion is solely due to the numerical truncation error. The magnitude of this dispersion depends on the size of the grid block and that of the time-step used in the numerical model. In a homogeneous medium, we find that the average macroscopic dispersivity changes little with distance. This observation can be seen in Fig. II-7, which shows variation in $\alpha_{\mathrm{MA}}$ with distance for two homogeneous cases. The only difference between the two cases is that one has 40 blocks in each direction and the other has 20 blocks in each direction. The magnitude of this dispersion is approximately $\Delta x / 2$, which is also the value expected from the theoretical results (Lantz, 1971). In Fig. II-7 dispersivities near the entrance are higher than the average in the homogeneous system. This behavior is due to boundary effects. In these analytical calculations to determine

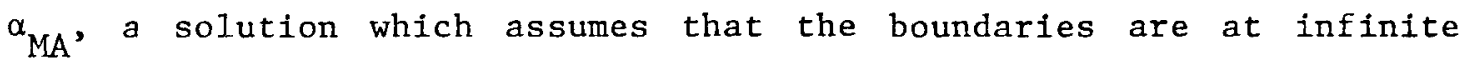
distance is used. However, the numerical simulator models a system with a finite length. Therefore, the dispersivities calculated near the entrance show this abnormal variation.

These results for a homogeneous medium show that by making proper correction for numerical dispersion, the physical macroscopic dispersion present in the permeable medium can be determined. In all 


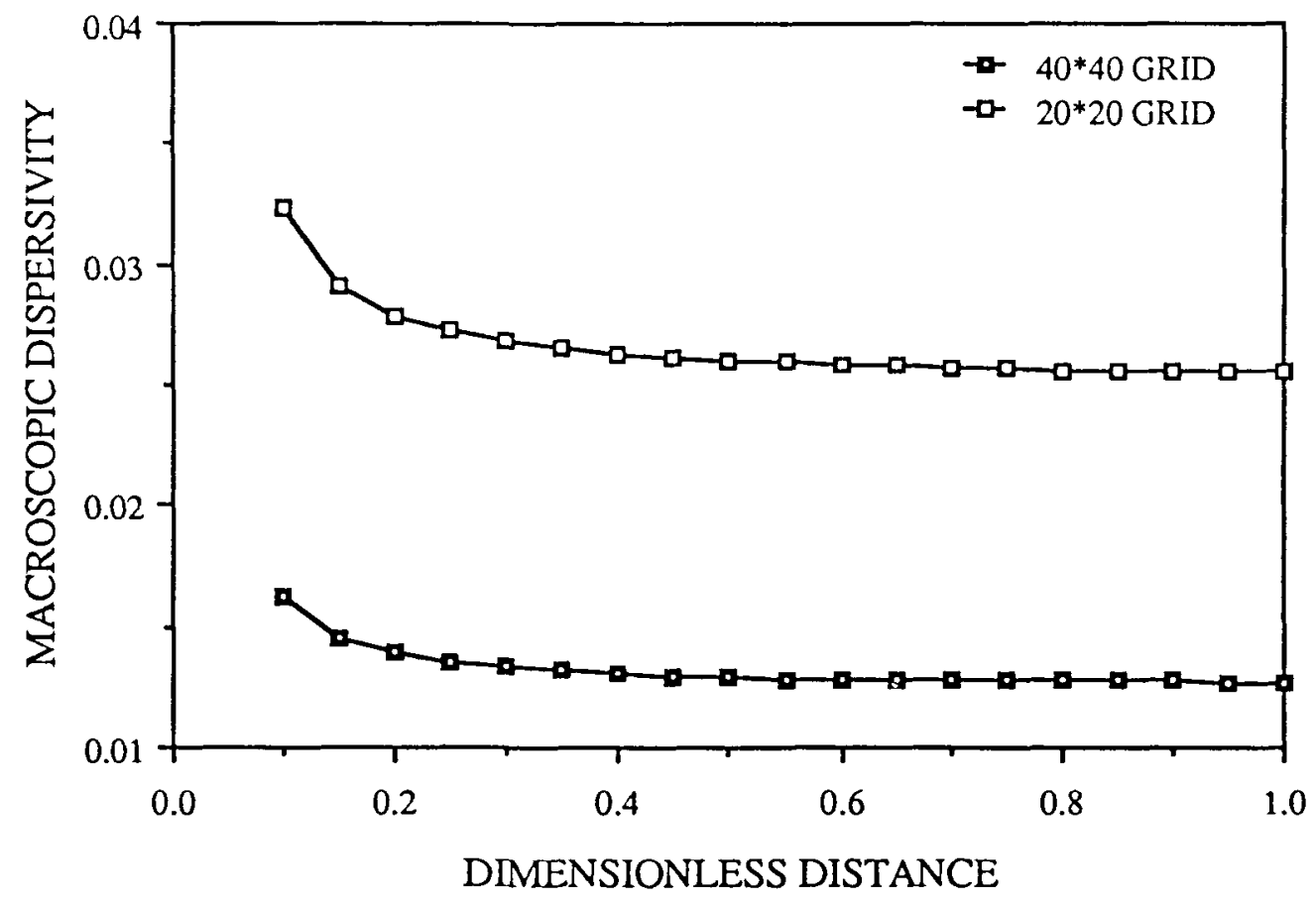

Figure II-7. Effect of grid-biock on macroscopic dispersivity, (homogeneous systems). 
subsequent results, this numerical dispersion components is subtracted from the total macroscopic dispersion to get the true value of $\alpha_{\mathrm{MA}}$.

Effect of Dykstra-Parsons Coefficient - In Fig. II-8, the average $\alpha_{M A}$ vs. distance for four values of Dykstra-Parsons coefficient is plotted. This figure is analogous to Fig. II-1 which shows variation of $\alpha_{\mathrm{ME}}$ with dimensionless time. In all the cases here the correlation length $\lambda_{D}$ is 0.025 . These runs have the same distribution of Heller points, but have different degrees of heterogeneity (which is obtained by changing the Heller's parameter $\alpha_{H}$ ). In Fig. II-8, the average $\alpha_{M A}$ values near the entrance are not shown because these averages are taken over a very few points (sometimes 3 to 5), and these results have very little meaning. From Fig. II-8, we can conclude that the macroscopic dispersivity is a function of $x_{D}$, specially for cases with high $V_{D P}$ values. In addition, the magnitude of the macroscopic dispersivity increases with $\mathrm{V}_{\mathrm{DP}}$, 1.e., a more heterogeneous medium has more local dispersion. The rate of variation of $\alpha_{M A}$ is also higher for cases with higher heterogeneity. This variation in macroscopic dispersivity with distance is due to the random heterogeneous nature of the permeable medium. The fluid travels faster through the more permeable portions of the reservoir. The net result of this behavior is the development of channels which start initially due to the local variation in velocity and are enhanced into instabilities at a larger scale. Some of this variation may also be due to the statistical error associated with calculating $\alpha_{M A}$ as described above.

In order to understand the nature of macroscopic dispersion, a 


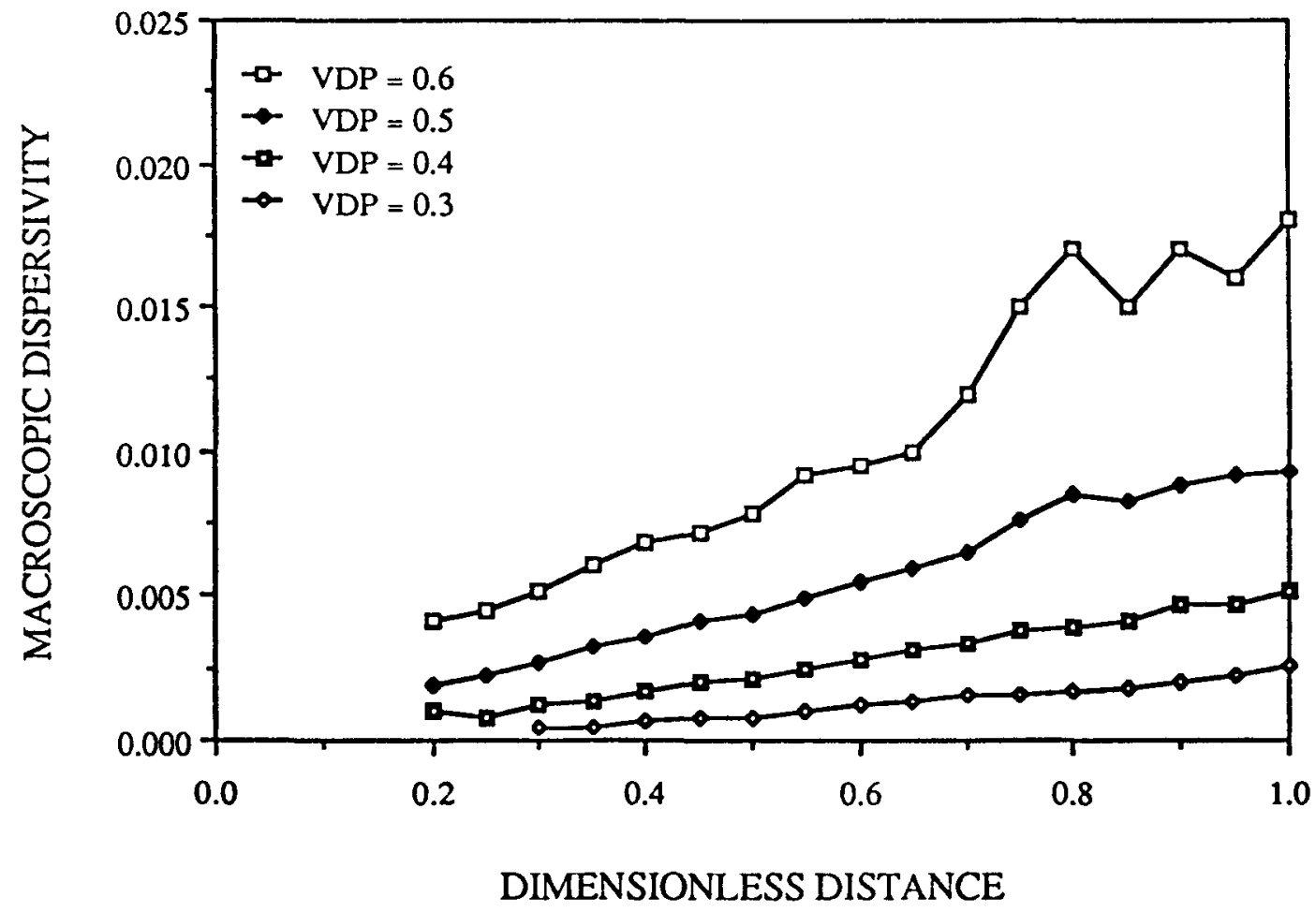

Figure II-8. Effect of Dykstra-Parsons coefficient on $\alpha_{M A}$. 
deeper look is taken into the displacement mechanism at the local scale. In Fig. II-9, three displacement profiles at different locations in the system are shown. These profiles represent the locally calculated macroscopic dispersivities across the cross-section. In this case the Dykstra-Parsons coefficient is 0.6 , the aspect ratio is one, and the correlation length is 0.025 . There is no molecular diffusion. If the permeable medium were homogeneous, this profile would be a straight line. However, because of the large degree of heterogeneity, there is a considerable amount of variation in $\alpha_{M A}$ values. In each case, the average value is represented by the solid vertical line. The average value increases as the fluid travels further into the medium. This increase in $\alpha_{M A}$ with distance is similar to the increase in $\alpha_{M E}$ with time. As discussed above, increasing dispersivities indicate a non-Fickian displacement. Another important feature of these $\alpha_{M A}$ profiles is that they give some idea about the location of high permeability regions where channeling initially develops. In these regions, the local (macroscopic) dispersivities are high and the fluid travels very quickly through them. The degree of variation in $\alpha_{M A}$ at any $x_{D}$ location also increases with distance. In addition the number of $\alpha_{\mathrm{MA}}$ values with large deviations from the mean also increase. As the total number of fluctuations as well as the magnitude of these fluctuations increases, the instabilities in the permeable medium also increase.

A comparison of local displacement characteristics between two heterogeneous permeable systems can also be made by studying local dispersivities. The macroscopc dispersivities $\alpha_{M A}$ 's for two $V_{D P}$ values of 0.8 and 0.4 at $x_{D}=0.5$ are shown in Fig. II-10. In both the 

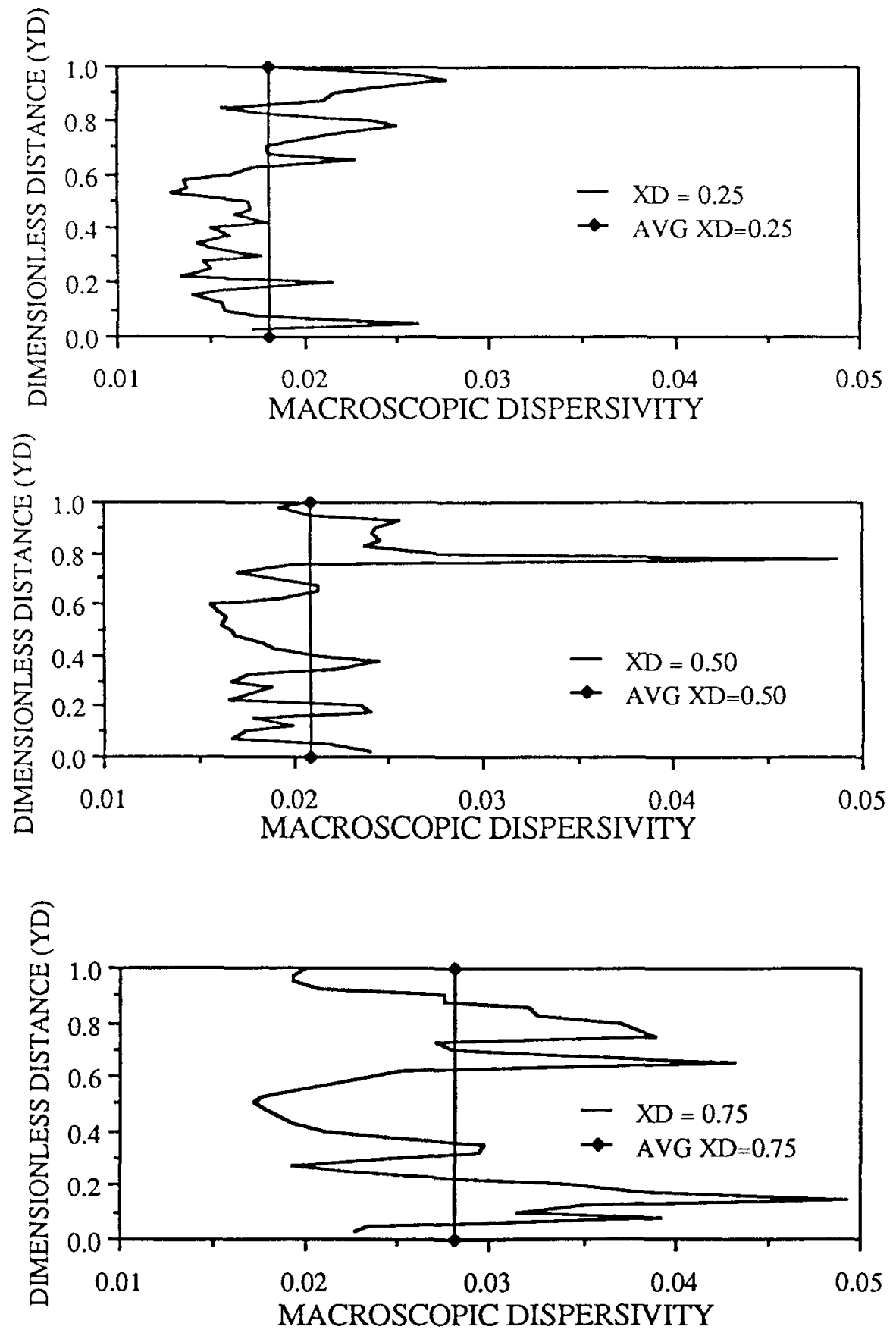

Figure II-9. Macroscopic dispersivities across the crosssections, $\mathrm{V}_{\mathrm{DP}}=0.6$. 


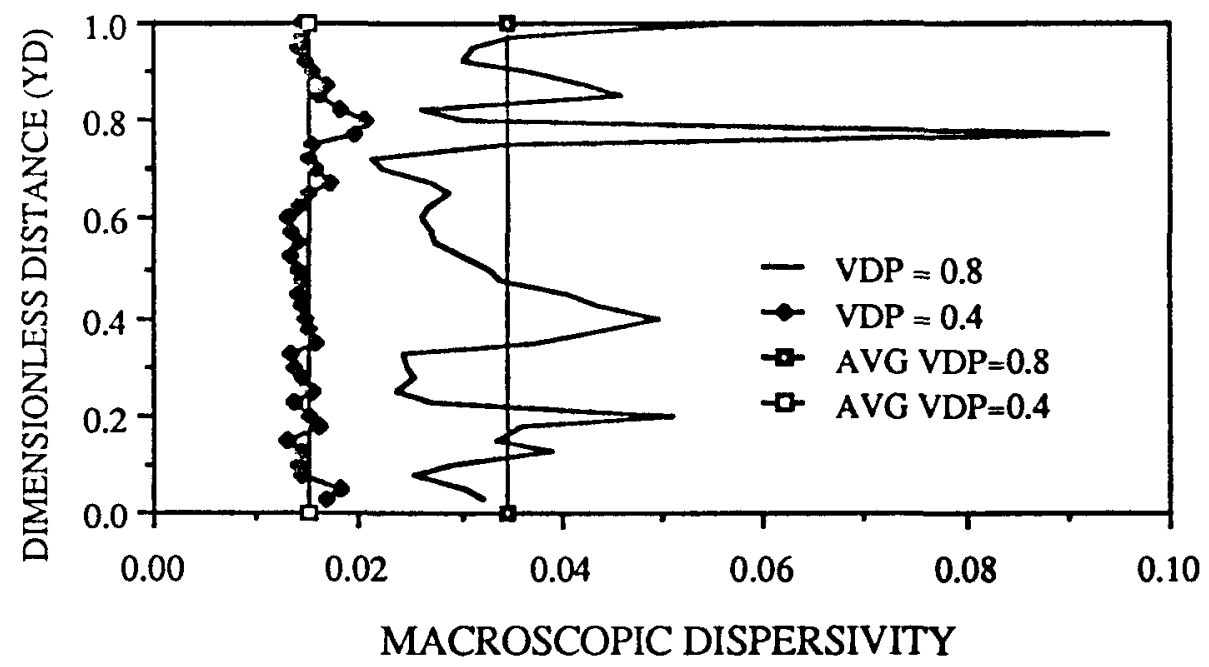

Figure II-10. Macroscopic dispersivities at $x_{D}=0.5$ for two $\mathrm{V}_{\mathrm{DP}}{ }^{\prime} \mathbf{s}$ 
cases, other variables such as aspect ratio and the correlation length are the same. The local $\alpha_{\mathrm{MA}}$ 's are nearly the same when $\mathrm{V}_{\mathrm{DP}}=0.4$, but at the higher $V_{D P}$ value of 0.8 there is a lot of variation in $\alpha_{M A}$ values. One local value for $\alpha_{\mathrm{MA}}$ is much higher than the others, indicating much larger local mixing than the average. This figure explains why displacement is uniform (Fickian) at low $\mathrm{V}_{\mathrm{DP}}$ values and channeling develops at high $V_{D P}$ values (as local instabilities grow to become megascopic instabilities). The average $\alpha_{\mathrm{MA}}$ is also higher for the case with the larger $V_{D P}$.

The megascopic and macroscopic dispersivities for these cases can also be compared. In Fig. II-11, $\alpha_{M A}$ and $\alpha_{M E}$ for two cases with $V_{D P}=0.6$ and $V_{D P}=0.4$ are shown. At any $V_{D P}$ the local (macroscopic) dispersivities are smaller than the corresponding megascopic values. However, when the degree of heterogeneity $\left(\mathrm{V}_{\mathrm{DP}}\right)$ decreases, the difference between the two dispersivities $\left(\alpha_{M A}\right.$ and $\left.\alpha_{M E}\right)$ also decreases. At high magnitudes of heterogeneity, there is a large difference between the megascopic and macroscopic dispersivities. This is a consequence of the scale effect. In a random permeable medium with heterogeneities approaching those in actual reservoirs ( $\mathrm{V}_{\mathrm{DP}} \geqq$ 0.6), the megascopic and macroscopic dispersivities would be significantly different. Thus, these results show that in general the dispersivities determined from displacement experiments on cores cannot be used to approximate field-scale $\alpha_{\mathrm{ME}}$ values. The core samples used in laboratory experiments are fairly homogeneous $\left(V_{D P} \cong 0.4\right)$ and have small correlation lengths. The typical oil reservoirs are fairly heterogeneous $\left(\mathrm{V}_{\mathrm{DP}} \geqq 0.6\right)$ and the correlation lengths can vary over a wide range. This observation has a direct consequence on miscible 


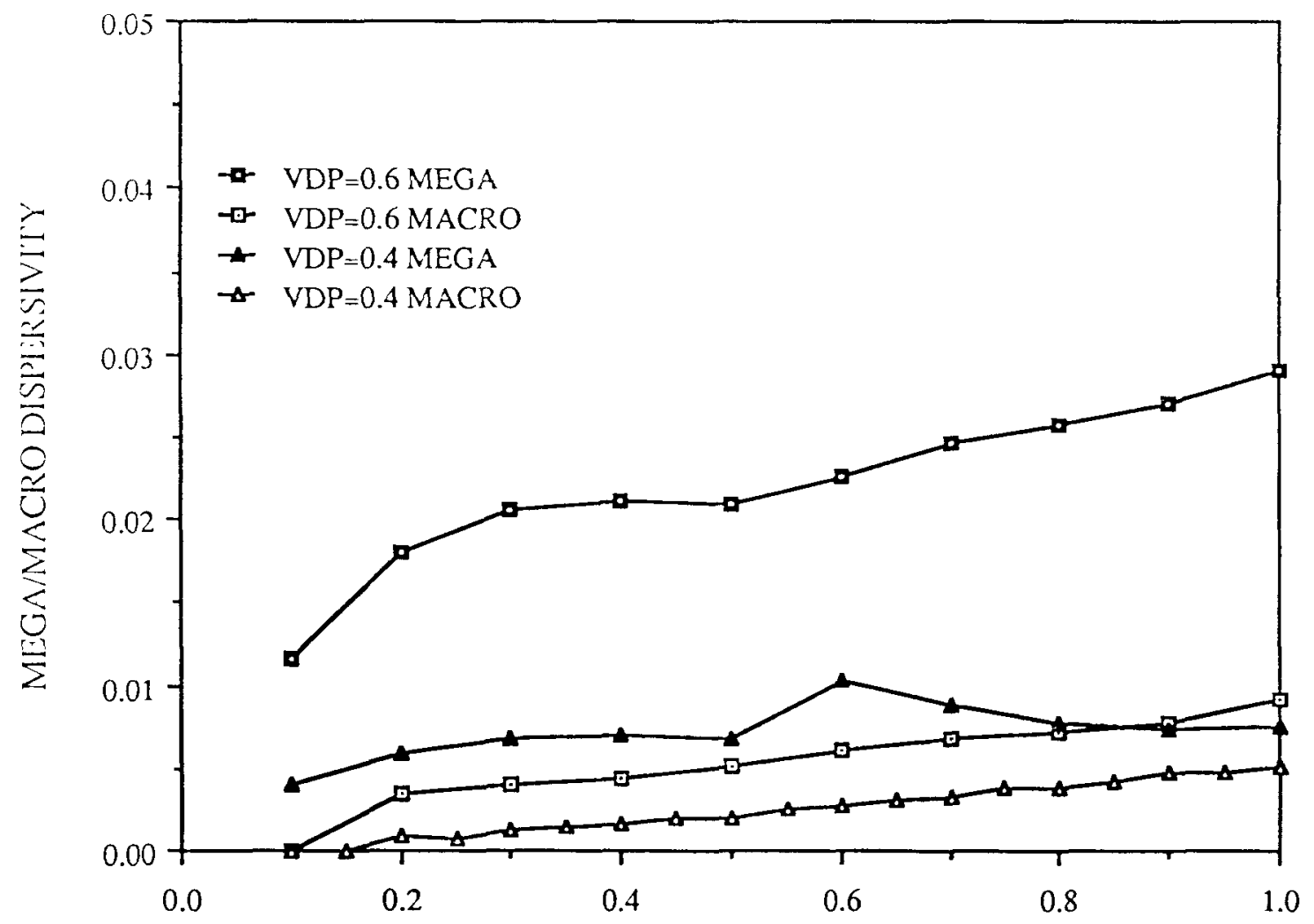

DIMENSIONLESS TIME/DISTANCE

Figure II-11. Comparison between megascopic and macroscopic dispersivities. 
displacements in oil fields where field type displacements (like pilot floods) are designed on the basis of displacement studies in cores.

Effect of Correlation Iength - Autocorrelation, a spatial property, is measured for a set of data distributed in the flow domain. On the contrary, macroscopic dispersivity is a local property measured at a point. Therefore, no specific relationship may exist between spatial correlation for flux/permeability and the macroscopic dispersivity. As described in the previous chapter, the megascopic dispersivity (which is measured at the same scale as correlation) follows specific trends depending on the degree of correlation in the spatial data. Somewhat different trends are observed in the macroscopic dispersion data. Figure II-12 displays both megascopic and macroscopic dispersivities for two sets of data with integral scales of 0.05 and 0.10 . Both sets have a $V_{D P}$ value of 0.6 , an aspect ratio of one and zero molecular diffusion. When the correlation length is long $\left(\lambda_{D}=0.10\right)$ megascopic dispersivity increases with time. The macroscopic dispersivity for this case with $\lambda_{D}=0.10$ also increases but the rate of increase is considerably smaller. In addition, the difference between $\alpha_{M A}$ and $\alpha_{M E}$ is large. In the second case, where $\lambda_{D}=0.05$, both $\alpha_{M A}$ and $\alpha_{M E}$ again increase but very slowly, and are nearly constant at late times/distances. Here, the difference between the two values ( $\alpha_{\text {MA }}$ and $\left.\alpha_{\mathrm{ME}}\right)$ is also smaller. This is due to the smaller correlation length and the near stable nature of the displacement.

These results again demonstrate the importance of the scale effect. In permeability distributions with large $V_{D P}(\geqq 0.6)$, there is not only a significant difference between megascopic and macroscopic 


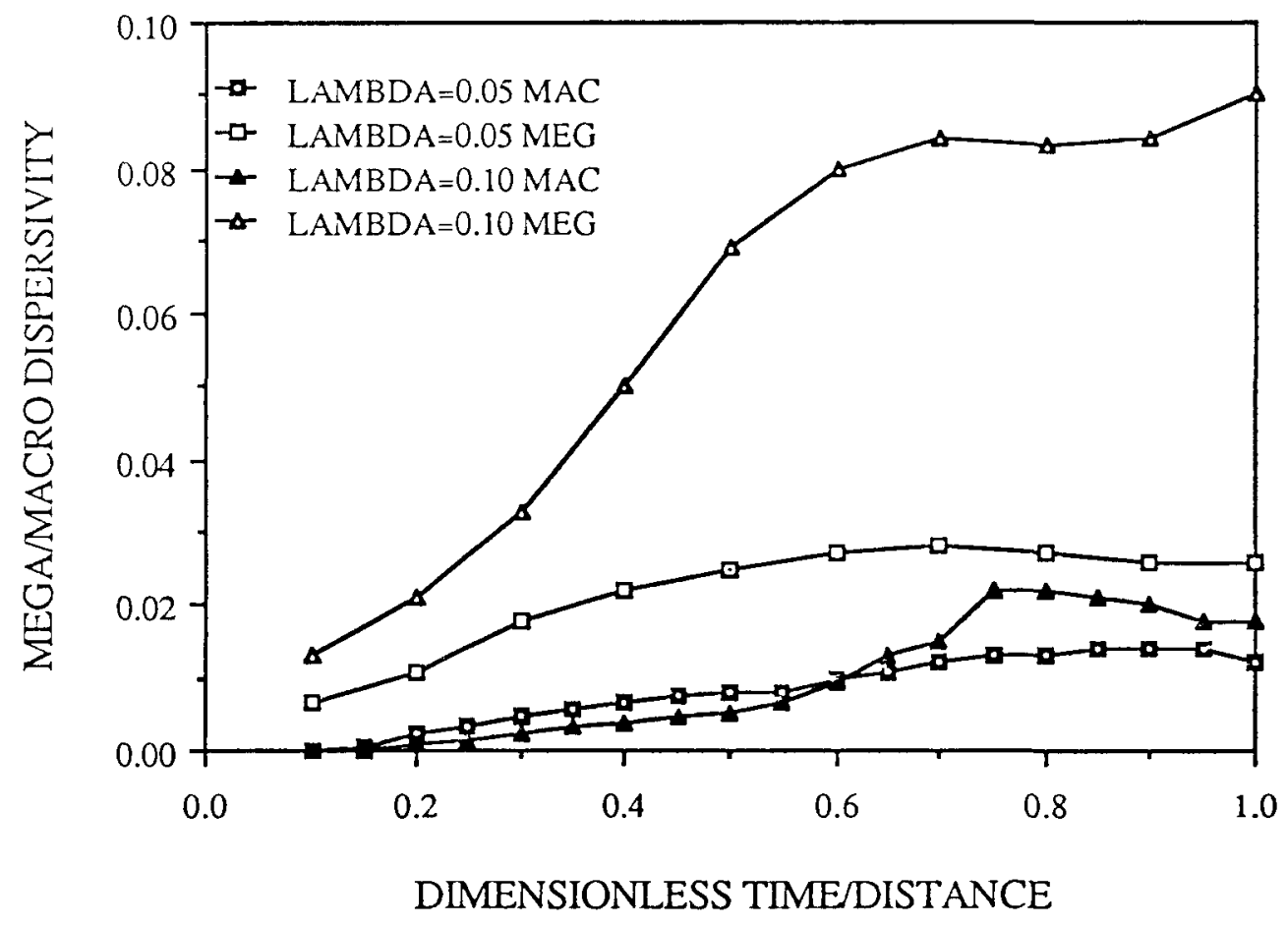

Figure II-12. Effect of correlation length on macro/megascopic dispersivities, $\mathrm{V}_{\mathrm{DP}}=0.6, \mathrm{~L} / \mathrm{W}=1$. 
dispersivities, but, in addition, the dispersivities measured at a local scale cannot completely account for regional (or spatial) phenomena such as correlation. The net result of large correlation in permeability (or flux) is time-dependent dispersivities. These time-dependent megascopic dispersivities are due to channeling of fluid through the more permeable regions of the reservoir. Thus, the locally measured macroscopic dispersivities cannot be used to model channeling since it is assumed that all mixing at the local scale is dispersive mixing. This observation also implies that macroscopic dispersivities would be independent of time and spatial position for purely dispersive flow.

The results for these cases with different Dykstra-Parsons coefficients and different correlation lengths give some insight into miscible displacement phenomena at macroscopic and megascopic scales. It can be concluded that:

1. In general the megascopic dispersivity is larger than macroscopic dispersivity. The difference between the two increases when $V_{D P}$ and $\lambda_{D}$ increase (Figs. II-11 and II-12) and vanishes when $\mathrm{V}_{\mathrm{DP}}$ is small.

2. Megascopic dispersivity $\alpha_{M E}$ is a strong function of correlation length but macroscopic dispersivity ${ }^{\alpha}{ }_{\mathrm{MA}}$ is a weak function, i.e., the rate of variation of dispersivity with $\lambda_{D}$ is much larger at the megascopic scale than at the macroscopic scale.

3. The instabilities at the macroscopic scale grow with the distance travelled (or dimensionless time) in a manner similar to the growth of instabilities at the megascopic scale. 
Effect of Aspect Ratio - In the previous work (Arya, 1986), it was shown that the aspect ratio is related to the spatial correlation, and the correlation changes depending on the manner in which the aspect ratio is changed. Specifically, we concluded that the rate of change of $\alpha_{Y A}$ with distance increases as the spatial correlation increases. This observation is also true here, because the aspect ratio of the system influences dispersivities only indirectly (through the spatial correlation).

Macroscopic dispersivities for four cases with aspect ratios of 1, 4, 5, and 10 are presented here. The same cases were analyzed in the previous chapter. In Fig. II-13, we observe that the variation of $\alpha_{\text {MA }}$ becomes very chaotic (random) at high aspect ratios. In all these cases $\mathrm{V}_{\mathrm{DP}}=0.6$ and no molecular diffusion is added. The spatial correlation of transport properties for non-unit aspect ratio cases depends on the arrangement of grid blocks in the simulation, as described in the previous chapter. When the aspect ratio is 5 and 10 , there are large spatial correlations because the arrangement of blocks in these two cases is the same as in the unit aspect ratio case. However, when the aspect ratio is 4 , the correlation length is the same as for $\mathrm{L} / \mathrm{W}=1$ because this case has proportionately more blocks (80 vs. 20) in the direction with the larger dimension. For aspect ratios of 5 and 10, the variations in $\alpha_{M A}$ are very large and do not follow any pattern. In addition, as in the previous section, the magnitude of average $\alpha_{\mathrm{MA}}$ is higher when the correlation length is large. One reason for these variations is the unequal number of points used to determine the average at every $x_{D}$ location. In addition, displacement in these two cases is very unstable and the injected 


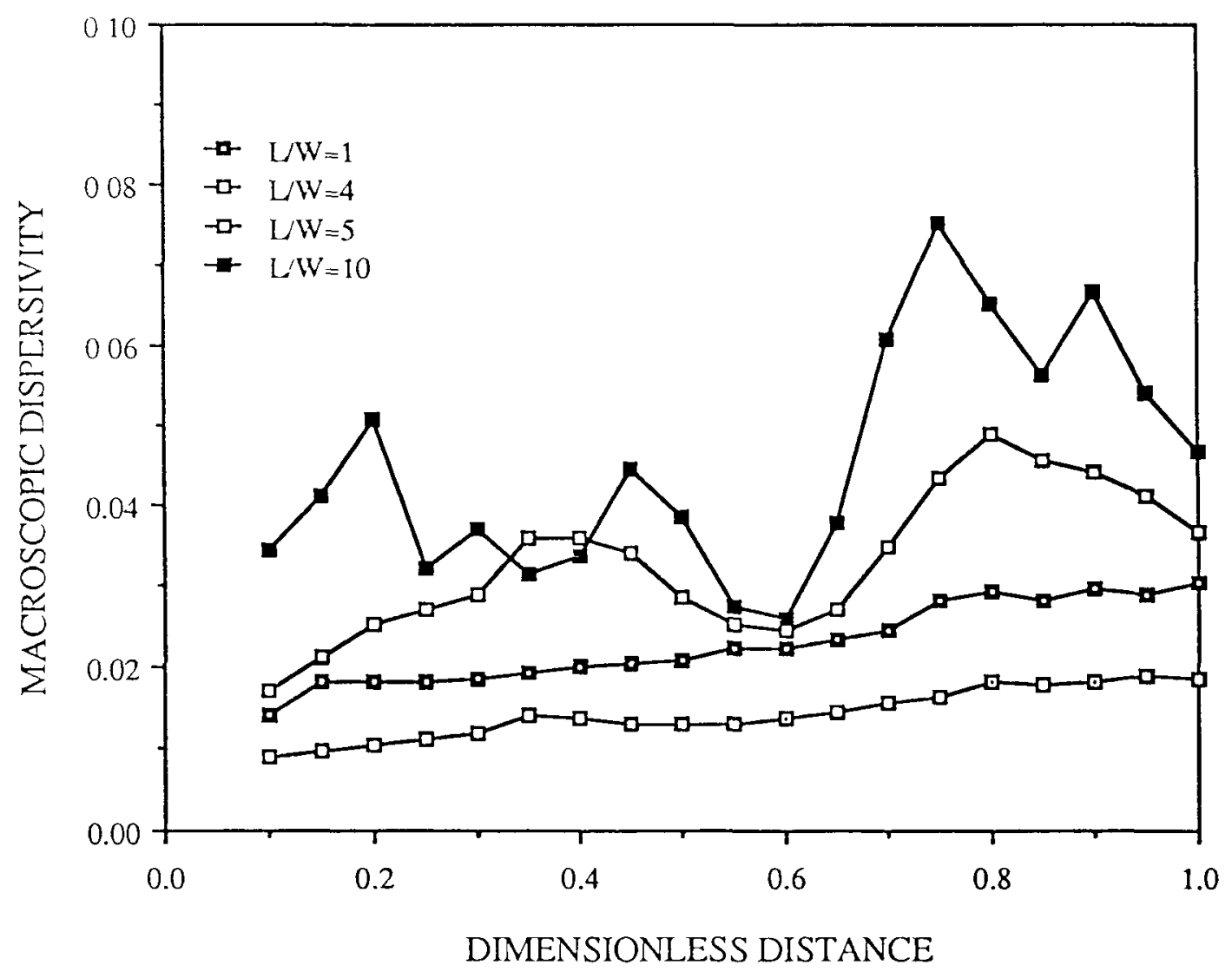

Figure II-13. Effect of aspect ratio on macroscopic dispersivities, $\mathrm{V}_{\mathrm{DP}}=0.6$. 
fluid channels very rapidly through the most permeable regions of the medium. This phenomenon has a significant influence on the macroscopic dispersivity and results in extreme (very Inw or high) macroscopic dispersivities. This local effect is not observed in the calculation of megascopic dispersivity because those values are obtained by averaging the concentrations across each cross-section and the extreme concentrations are averaged out.

The channeling and unstable nature of the displacements in two cases with large aspect ratios (5 and 10) can be determined by analyzing the behavior of local $\omega_{\text {NA }}$ ralues at different cross-sections. In Fig. II-14, three $\alpha_{\text {:A }}$ profiles for the aspect ratio of 5 are shown. These megascopic dispersivity profiles are at $x_{D}$ values of $0.25,0.50$ and 0.75 . The random nature of $\alpha_{M A}$ is evident; even the average values at each $x_{D}$ do not follow any pattern. From the previous work we recall that permeable media with large aspect ratios behave like layered systems. This behavior controls the macroscopic dispersivities significantly. Each location at a cross-section behaves like a non-communicating layer and the $\alpha_{M A}$ values fluctuate by large amounts. The same behavior is observed in Fig. II-15, which shows local dispersivities for the aspect ratio of 10 . In this case, at $\mathrm{x}_{\mathrm{D}}=0.75 \alpha_{M A}$ values at only some locations of the cross-section are shown. The macroscopic dispersivities at the remaining locations cannot be determined due to the reasons discussed earlier. In both these cases $V_{D P}=0.6$. These profiles confirm the observation that a randomly heterogeneous medium behaves like a layered medium with large aspect ratio. These pseudo layers do not communicate and the displacement is controlled by large convective fluxes in the primary 

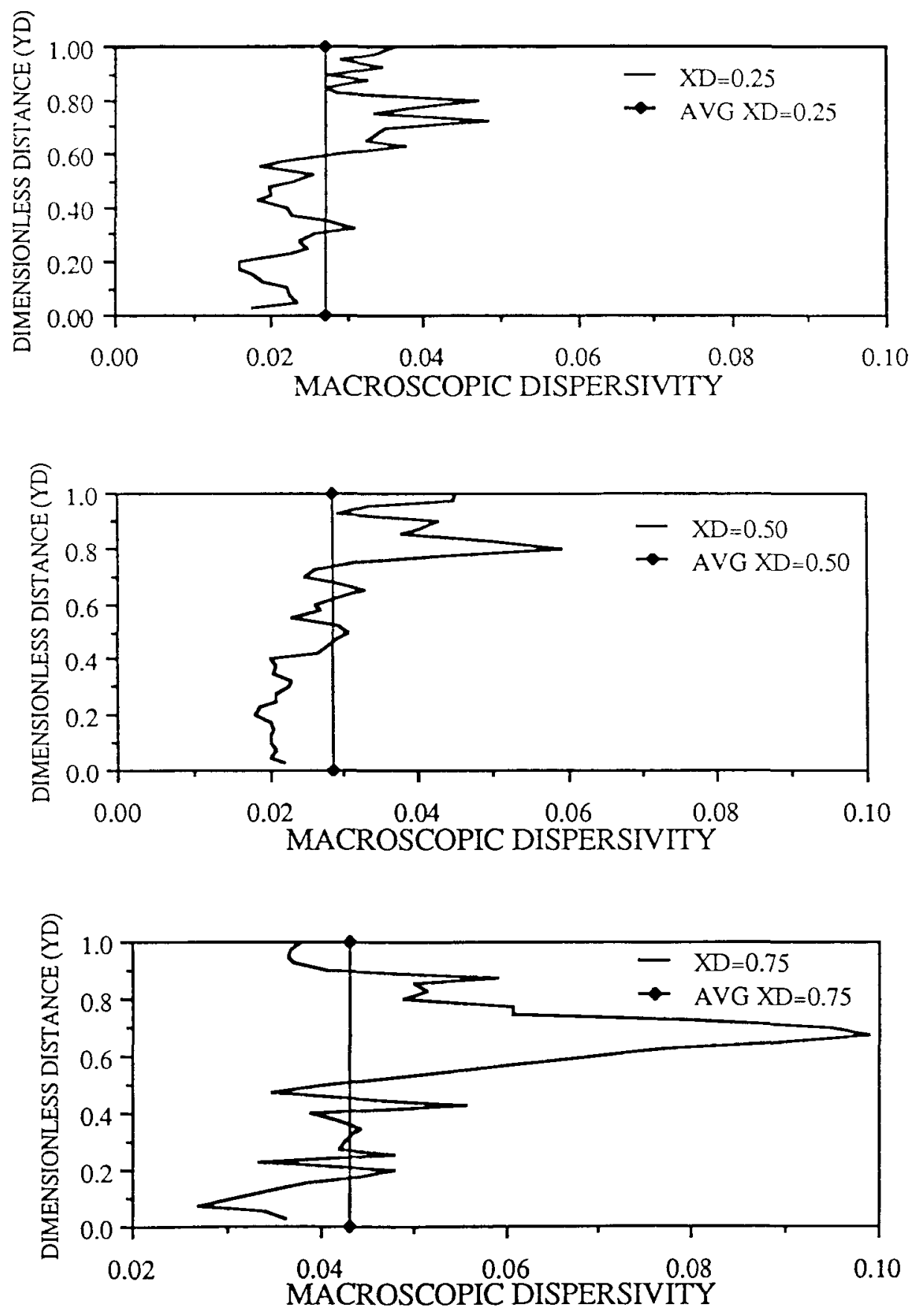

Figure II-14. Macroscopic dispersivities at three crosssections, $V_{D P}=0.6, L / W=5$. 

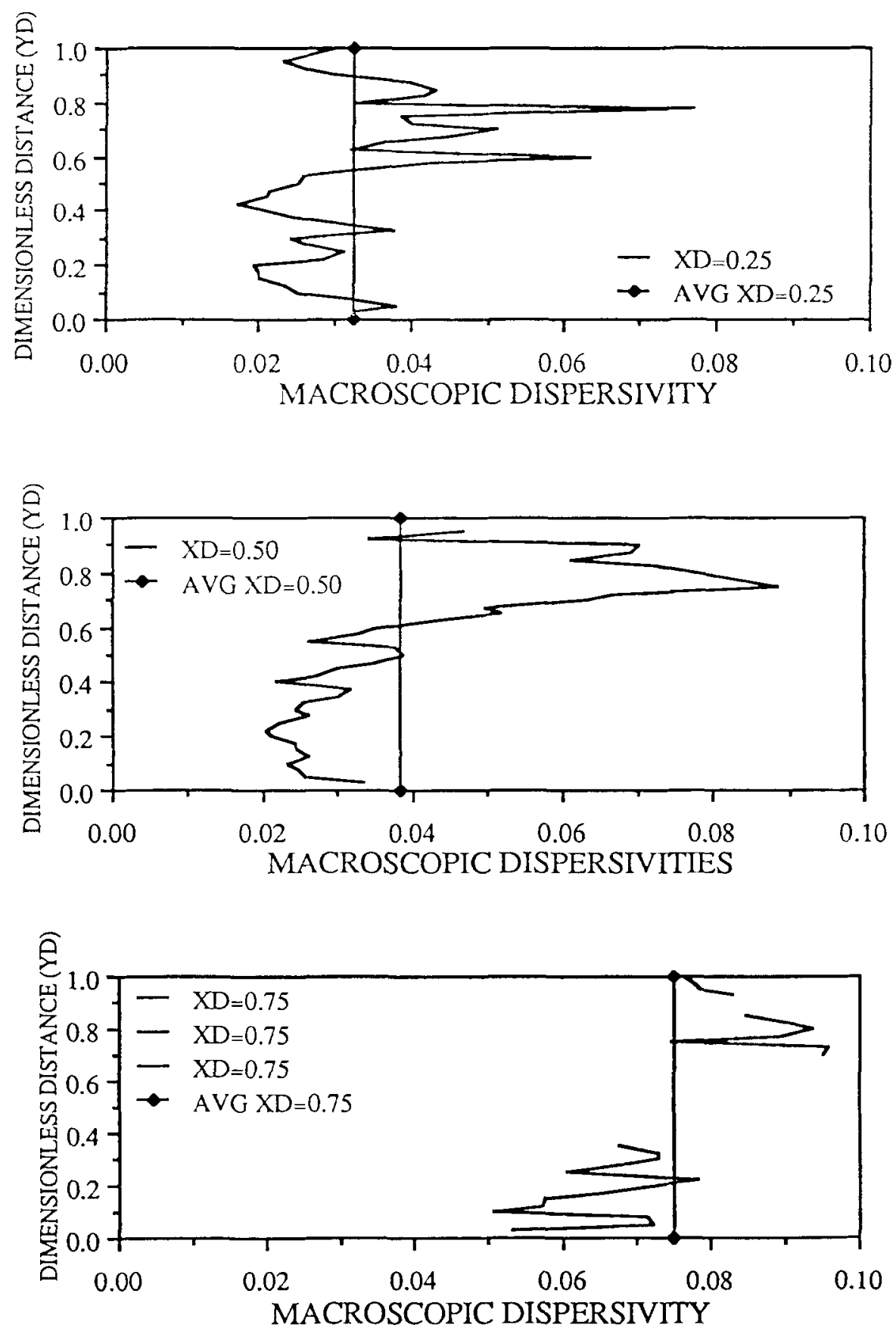

Figure II-15. Macroscopic dispersivities at three crosssections, $\mathrm{V}_{\mathrm{DP}}=0.6, \mathrm{~L} / \mathrm{W}=10$. 
direction of flow (parallel to the $\mathrm{x}$-axis).

One possible way to evaluate these variations in local $\alpha_{M A}$ values is by calculating the standard error for the $\alpha_{M A}$ values at each location. The standard error at 95 percent confidence leve 1 is defined as $\alpha \pm 1.96 \sigma / \sqrt{n}$ where $\sigma$ is the standard deviation for that set and $n$ is the number of points in the set. Thus, the standard error takes into account the nonuniform number of points for calculating averages. Figure II-16 shows that the standard error increases with the aspect ratio. This figure shows the average $\alpha_{M A}$ and the $\alpha_{M A} \pm$ standard error values for aspect ratios of 1 and 10 . The standard errors for $L / W=10$ are much larger indicating the large variations in the local average values of $\alpha_{\mathrm{MA}}$ 's.

From these results we conclude that if $\alpha_{M A}$ is very high and if there are large variations in the cross-sectionally averaged values, there is a good possibility that the displacement is unstable with little or no vertical communication. In these cases, it is a good idea to examine the local distribution of $\alpha_{M A}$ 's. The variation in $\alpha_{M A}$ at any fixed cross-section can help in locating unstable displacements through high permeability regions.

Effect of Diffusion - The effect of adding molecular diffusion to macroscopic dispersion is similar to that for megascopic dispersivity. With the addition of a diffusion component the total flux increases by the same amount in both the directions. However, the relative magnitudes of the convective and dispersive fluxes change and the ratios of the $x$ and $y$ flux depend on the amount of diffusion added to the system. One consequence of this additional component is that there 


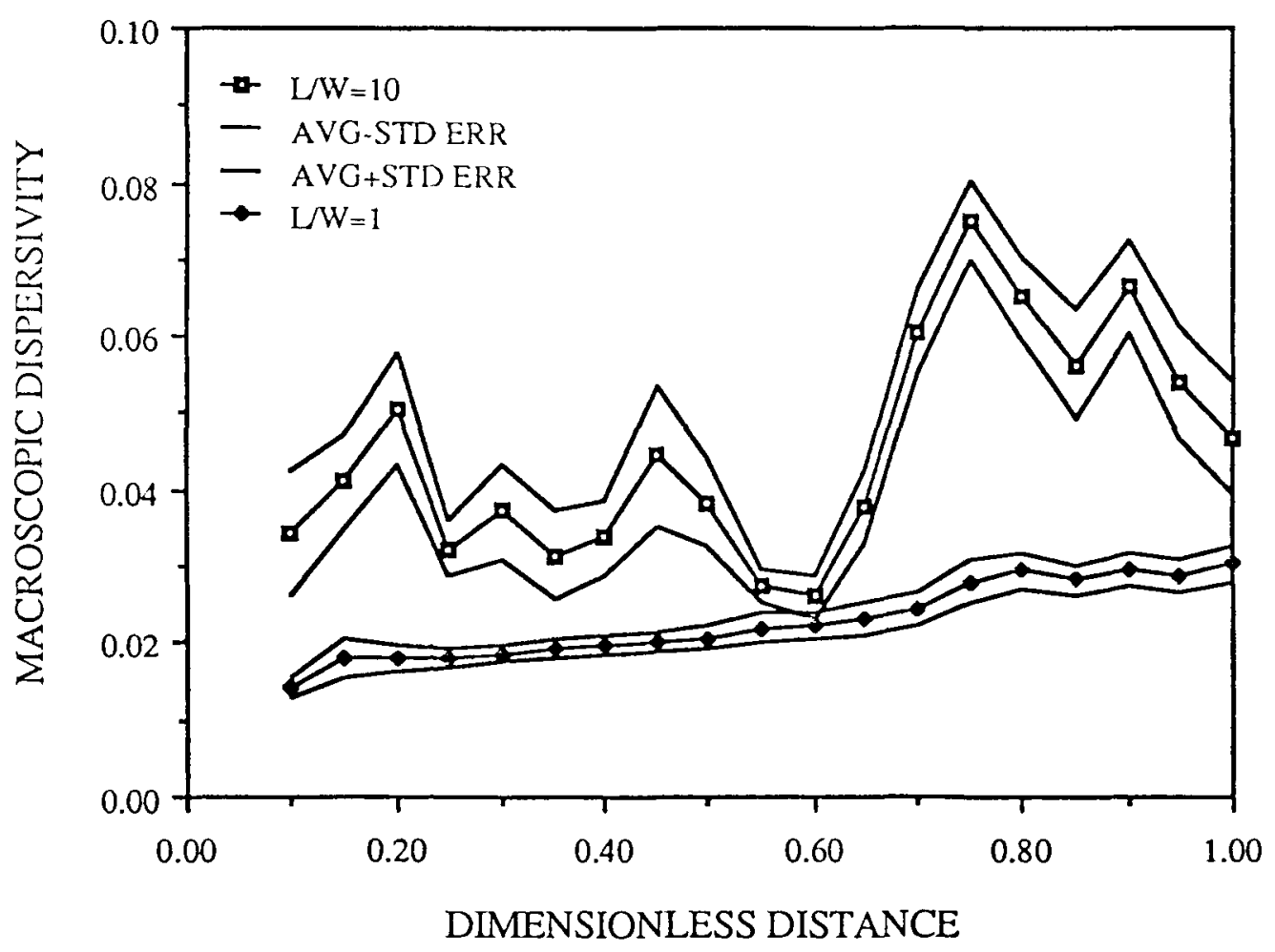

Figure II-16. Standard error in average macroscopic values, $\mathrm{v}_{\mathrm{DP}}=0.6$. 
is enhanced mixing in the direction normal to flow. Because of this enhanced mixing the fluid travels more uniformly through the permeable medium and the variation in macroscopic dispersivity at any cross-section is much lower.

In this section first the effect of adding diffusion on fluid transport in homogeneous medium is analyzed. We give results in terms of the dimensionless diffusion coefficient

$$
\mathrm{D}=\frac{\mathrm{D} o \phi}{\mathrm{uL}}
$$

where Do is the molecular diffusion coefficient. Figure II-17 shows the change in $\alpha_{M A}$ with distance in a homogeneous system at several levels of diffusion. In all the cases the aspect ratio is one. The total macroscopic dispersion is due to molecular diffusion and numerical truncation error. The $\alpha_{M A}$ values are independent of $x_{D}$ at lower values of diffusion. At higher values of diffusion, the macroscopic dispersivity increases with distance for a short distance before becoming constant. This behavior is due to the very high diffusion, the total dispersivity is very high and the Peclet number is very low. And at low values of Peclet number, the solution to the C-D equation with boundaries set at infinite distance (which is used here) has a large error component. At low Peclet numbers the size of the mixing zone is as large or larger than the size of the finite system modeled in the finite-difference analog. Therefore, additional terms are required in the analytical solution to account for the boundary effects. As discussed earlier this problem was corrected in the calculation of $\alpha_{M E}$ by using an analytical solution derived with proper boundary conditions. 


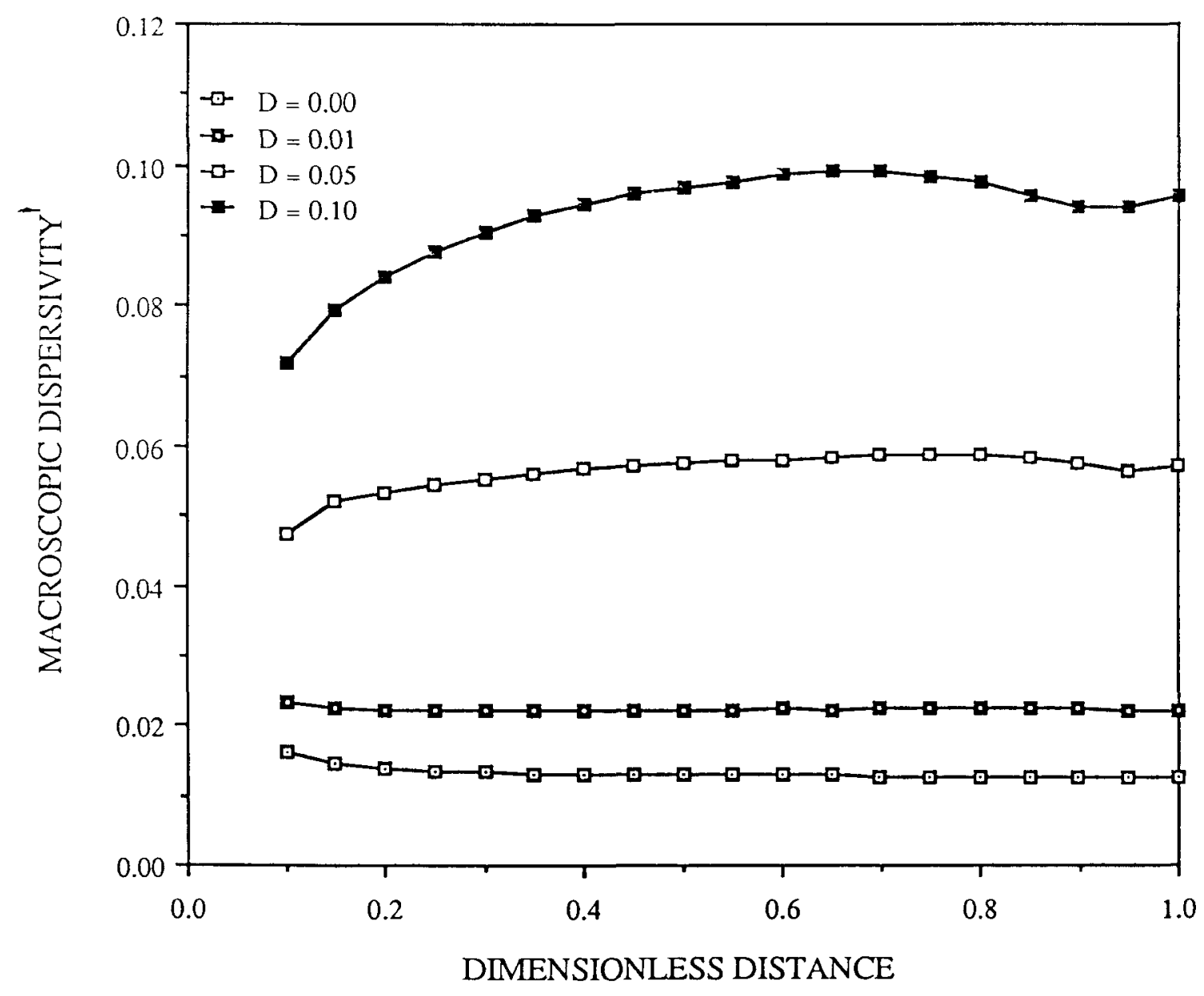

Figure II-17. Effect of diffusion on macroscopic dispersivities, (homogeneous system). 
Similar conclusions can be drawn from Fig. II-18, which shows the effect of diffusion on $\alpha_{M A}$ for a heterogeneous system with $V_{D P}=0.6$. In this case there is an additional physical dispersion component present due to the permeability heterogeneity. Thus, the total calculated macroscopic dispersivity is higher than that for homogeneous systems. In all these cases the same permeability distribution was used and the aspect ratio was one.

Due to the large $\alpha_{\mathrm{MA}}$ values, the boundary effects discussed in the previous paragraph become important and the dispersivities are not constant. In fact the channeling tendency is significantly reduced, as confirmed by the dispersivfty profiles. These profiles in Fig. II-19, are for three cases with different levels of diffusion and are calculated at $x_{D}=0.5$. In the absence of diffusion $(D=0.0)$, there is evidence of channeling, as indicated by fluctuations in $\alpha_{\text {MA }}$ values. One major channel can be seen developing near the upper edge of the system. With the addition of some diffusion $(D=0.01)$, the degree of transverse mixing increase considerably and the $\alpha_{\mathrm{MA}}$ profile indicates nearly uniform displacement. With more diffusion $(D=0.05)$, the profile is more uniform and no instabilities are present. The growth of the unstable channel has been controlled by better transverse mixing.

The same conclusions can also be drawn by observing the concentration histories at any cross-section. In Fig. II-2, concentration histories at $\mathrm{x}_{\mathrm{D}}=0.5$ are shown, for a case with $\mathrm{V}_{\mathrm{DP}}$ $=0.6$ and no molecular diffusion. In Figs. II-20 and II-21, the concentration histories for the same case with $D=0.01$ and $D=0.05$ are displayed. With the addition of diffusion, the width of the band over which the curves are spread out becomes smaller. This indicates that 


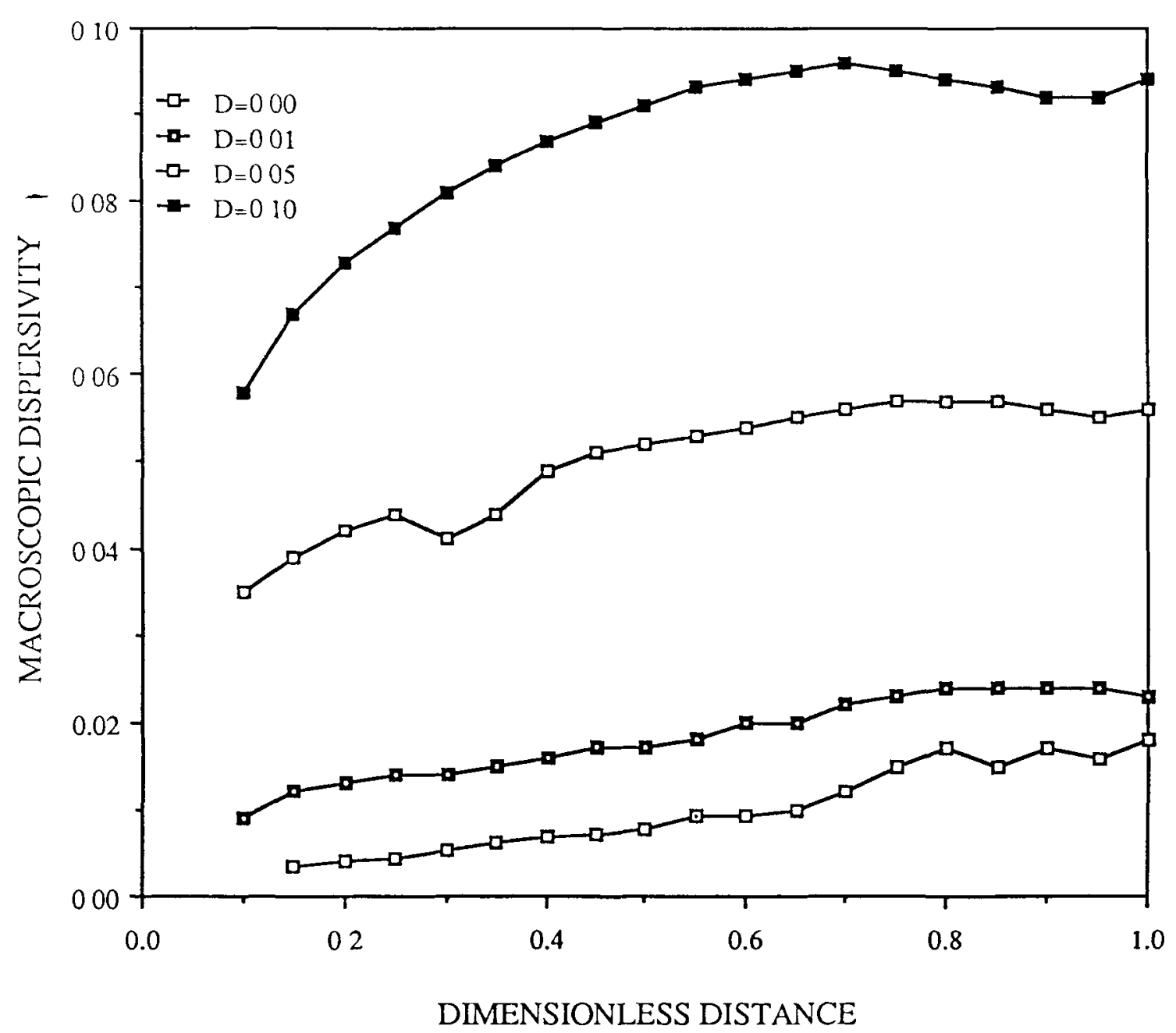

Figure II-18. Effect of diffusion on macroscopic dispersivities, $\mathrm{V}_{\mathrm{DP}}=0.6$ 


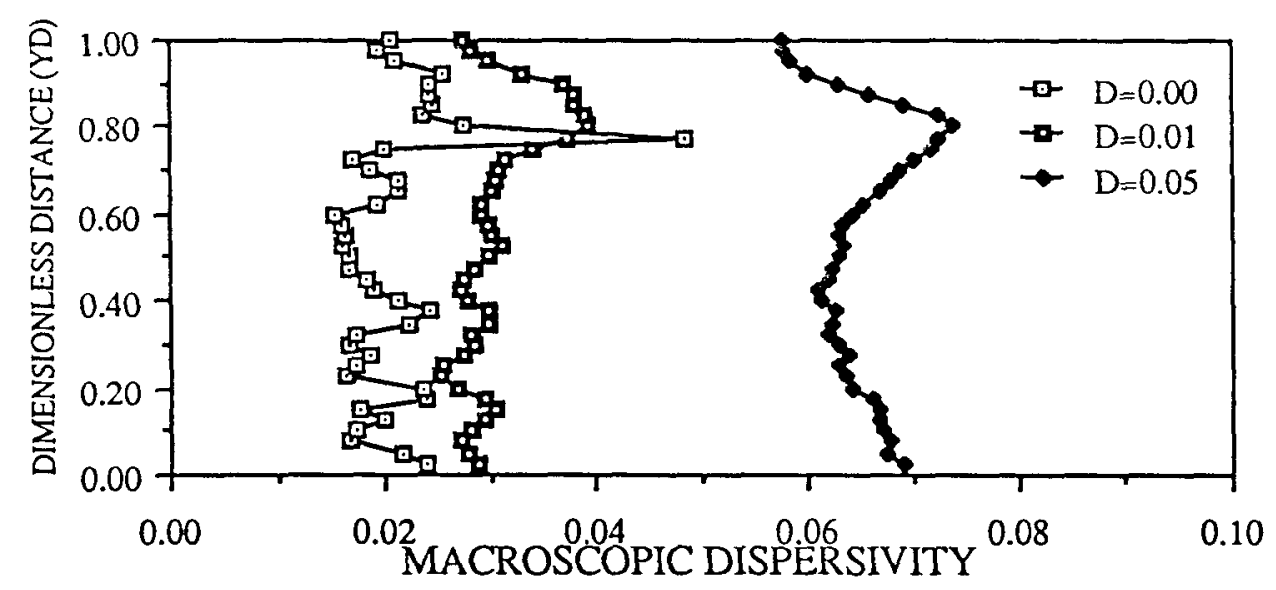

Figure II-19. Effect of diffusion on macroscopic dispersivities at a cross-section, $x_{D}=0.5$. 


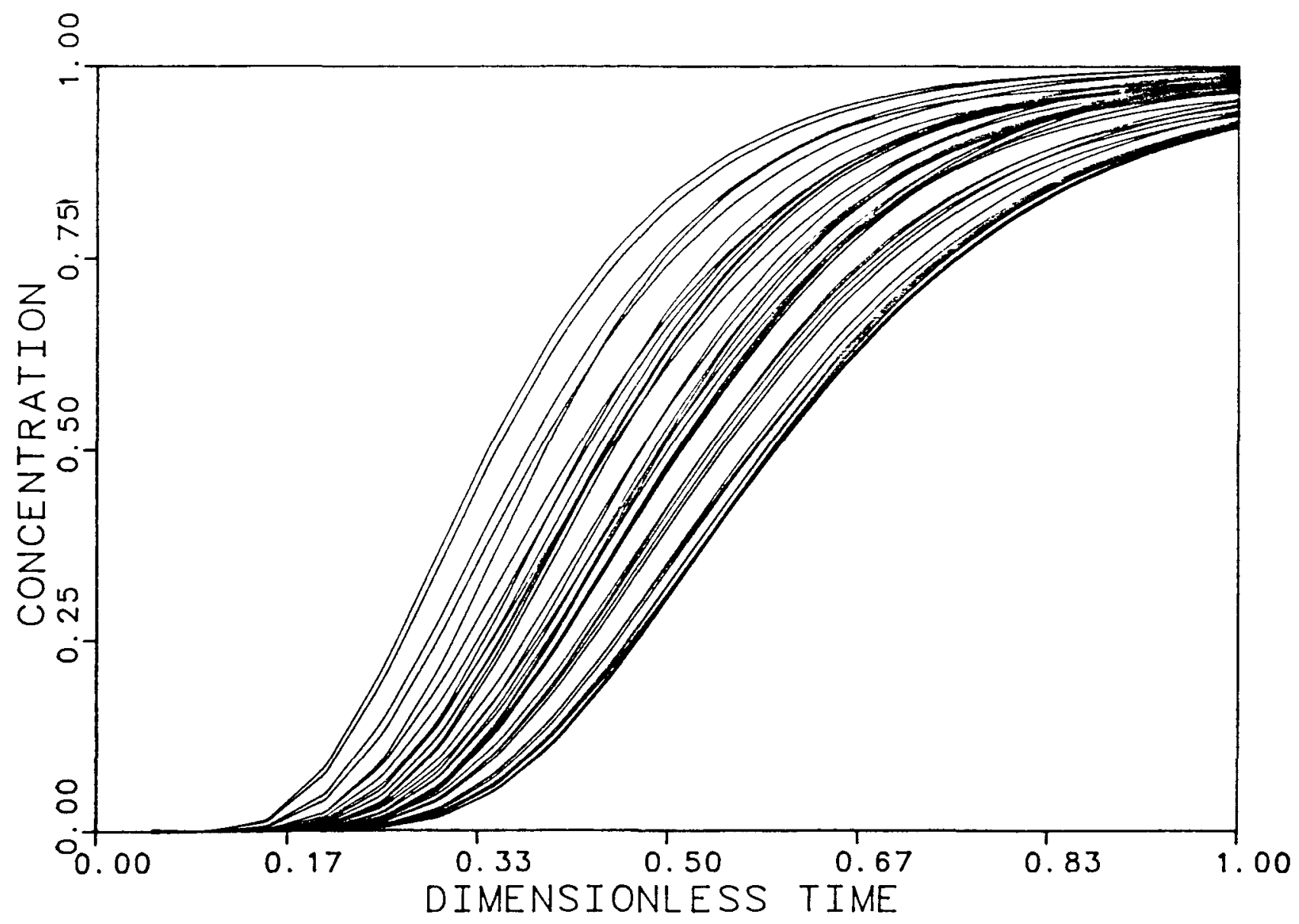

Figure II-20. Effect of diffusion on concentration histories $\mathrm{V}_{\mathrm{DP}}=0.6, \mathrm{~L} / \mathrm{W}=1, \mathrm{D}=0.01$ 


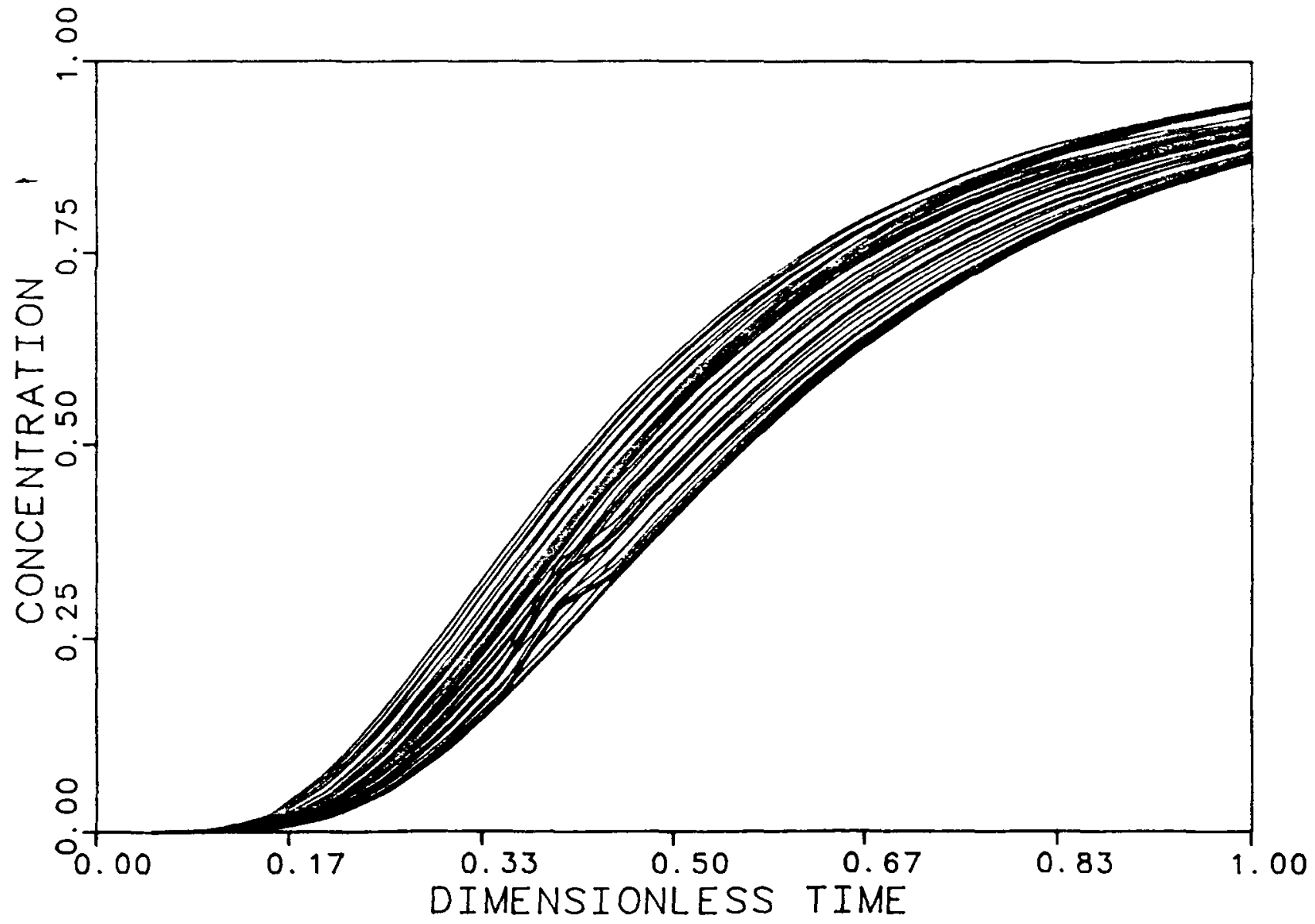

Figure II-21. Effect of diffusion on concentration histories,

$$
\mathrm{V}_{\mathrm{DP}}=0.6, \mathrm{~L} / \mathrm{W}=1, \mathrm{D}=0.05
$$


a11 concentrations are travelling close to each other and more transverse flow is taking place.

Thus, just as in the case of megascopic dispersion, diffusion stabilizes the displacement with enhanced transverse mixing at the macroscopic scale also. These results also point out the importance of using proper boundary conditions in the solutions for the $C-D$ equation. The results can be completely misinterpreted (for example non-Fickian flow instead of Fickian flow), by using an incorrect analytical solution.

Effect of Anisotrony - One measure of the degree of lateral communication in a permeable medium is the magnitude of the cross-flow index, $R_{L}$. The megascopic dispersivities for all systems with same cross-flow jndex behave alike because of the inherent averaging present at the megascopic scale. However, at the macroscopic scale, the dispersivities are calculated at individual points and the dispersivity values can vary widely between adjacent points depending on the degree of communication present. The macroscopic dispersivity as presented here is the average value for all locations across a cross-section at any $x$ position. If there is very little or no vertical communication present, these values can differ substantially from one point to the next across the cross-section. On the other hand, if the vertical communication is extremely good (as in the case of vertical equilibrium), all the values across the cross-section should fall within a narrow range (i.e., very small standard deviation). In such a case the cross-flow index, $R_{L}$, is also high. 
Results for two cases, one with a low $\mathrm{R}_{\mathrm{L}}=0.1$ and the other with a high $R_{L}=5$ confirm the observations made in the preceeding paragraph. In Fig. II-22, the megascopic dispersivities for two cases with $R_{L}=0.1$ are presented. One case has unit aspect ratio with some vertical communication $\left(k_{y} / k=0.01\right)$ and the other case has an aspect ratio of 4 and almost no vertical communication $\left(k_{y} / k=0.000625\right)$. In both cases $\mathrm{V}_{\mathrm{DP}}$ is 0.6 . The $\alpha_{\mathrm{MA}}$ values are nearly constant when the aspect ratio is large $(\mathrm{L} / \mathrm{W}=4)$, but increase with distance when the aspect ratio is $\operatorname{small}(\mathrm{L} / \mathrm{W}=1)$. The degree of cross-flow is small in both the cases. However, when the aspect ratio is 4 there are proportionately fewer blocks in the y-direction. This results in a lower value of $\lambda_{D}$ and the channeling tendency is retarded. Some explanation for this difference in the $\alpha_{M A}$ variation for these two cases with the same $R_{L}$ can be offered by analyzing the local variations in $a_{M A}$ in Fig. II-23. This figure shows more variations from the mean when $L / W=1$ than for the case with $L / W=4$, even though it $(\mathrm{L} / \mathrm{W}=4$ case) has a lower vertical permeability. This apparent contradiction is due to the strong influence of lateral boundaries on the displacement. When the boundaries are very close $(L / W=4)$, the channeling tendency is retarded and the displacement is more stable than in the case where both the lateral and longitudinal dimensions are equal.

When the vertical communication is high $\left(R_{L}=5.0\right)$, similar trends in $\alpha_{M A}$ values are observed. As seen in Fig. II-24, there is no trend in the variation of $\alpha_{\mathrm{MA}}$ with distance and both the cases follow each other more closely. One of these runs has an aspect ratio of 1 and $k_{y} / k=25$, and the other case has $L / W=5$ and $k_{y} / k=1$. The $\alpha_{M A}$ 


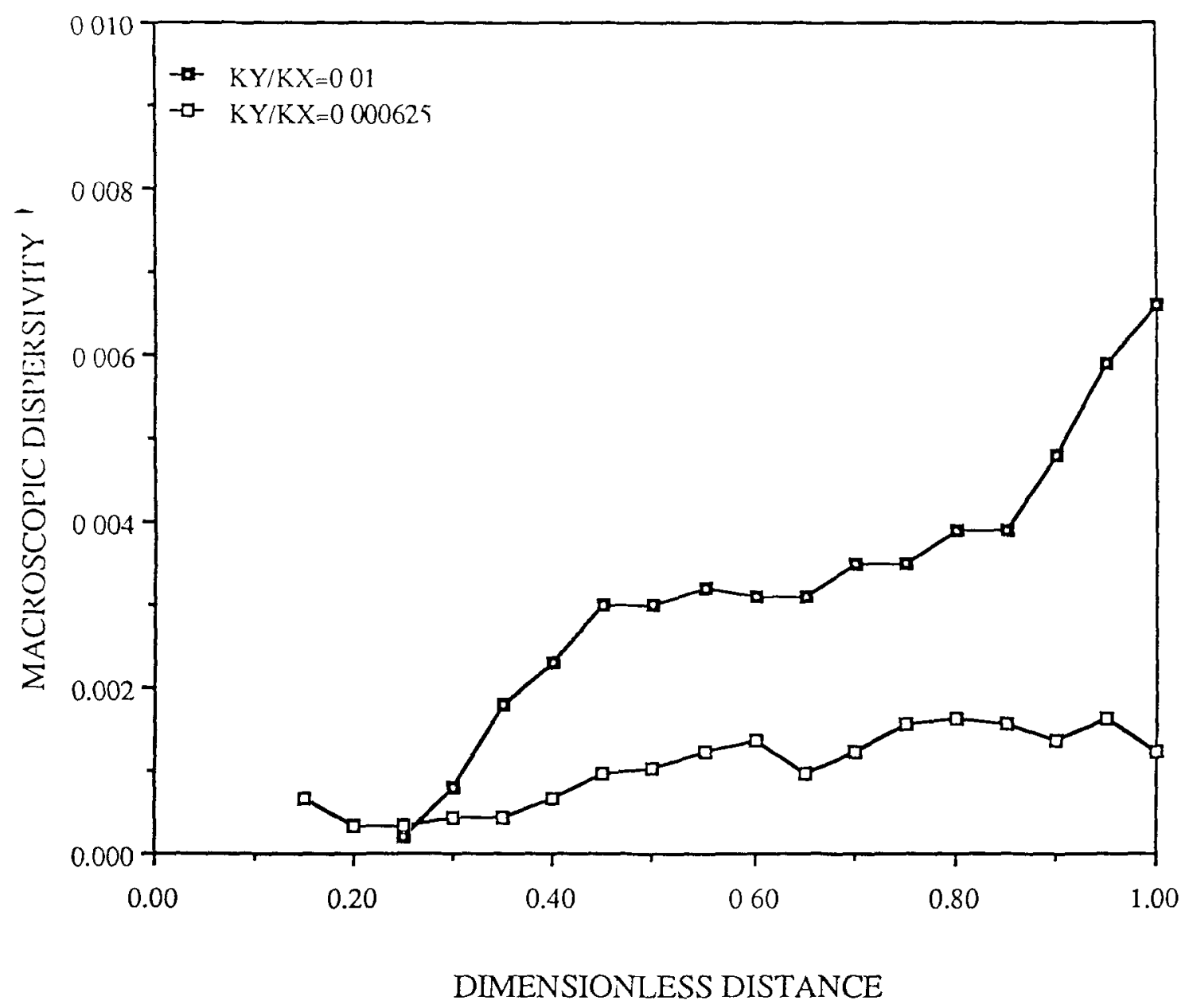

Figure II-22. Effect of anisotropy on macroscoptc dispersivities, $\mathrm{V}_{\mathrm{DP}}=0.6, \mathrm{R}_{\mathrm{L}}=0.1$ 


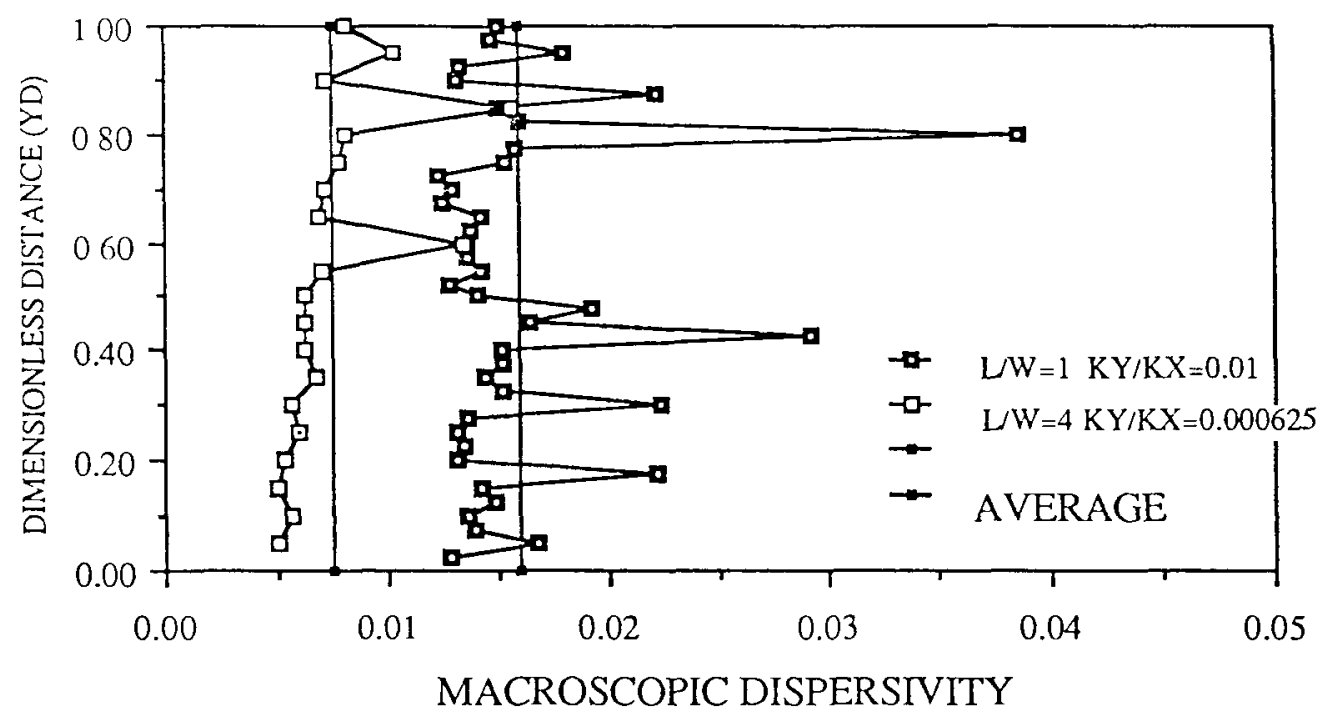

Figure II-23. Macroscopic dispersivities at a cross-section, $v_{D P}=0.6, x_{D}=0.5, R_{L}=0.1$ 


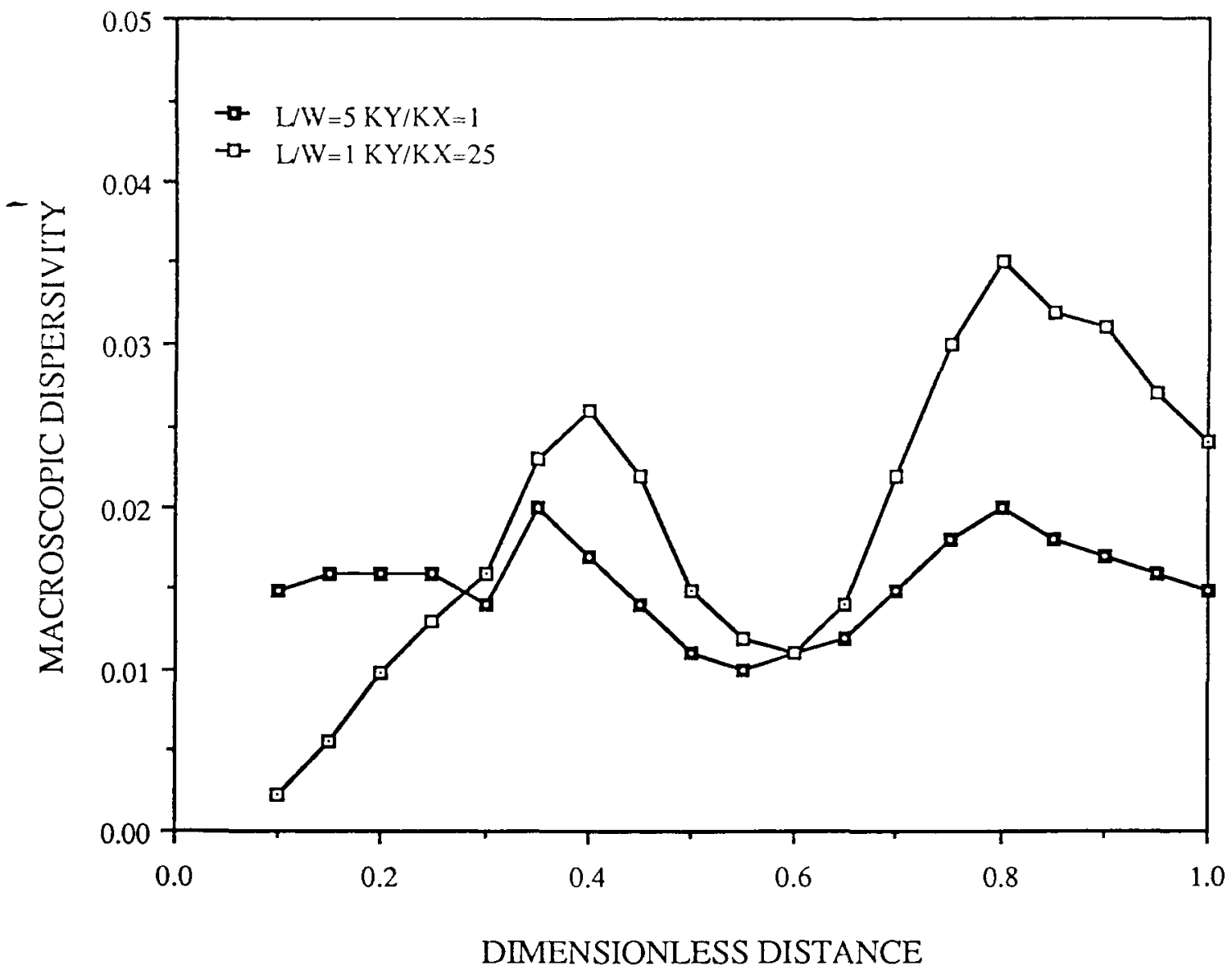

Figure II-24. Effect of anisotropy on macroscopic dispersivities, $\mathrm{V}_{\mathrm{DP}}=0.6, \mathrm{R}_{\mathrm{L}}=5.0$ 
values for both these cases vary with distance. These variations are a consequence of unstable flow. The local variations in $\alpha_{\mathrm{MA}}$ can be observed in Fig. II-25, which shows the point values of $\alpha_{M A}$ at $x_{D}$ $=0.5$.

These local $\alpha_{M A}$ values are nearly identical across the entire cross-section for these two cases. These profiles indicate unstable flow in a small section. The effect of lateral boundaries is once again evident here. Even though there is very high degree of crossflow, the two dimensions are equal and the instabilities travel faster than the enhanced mixing due to crossflow.

These results indicate that the dimensions of the system together with the degree of crossflow present have a considerable influence on local displacement. In general, large vertical communication does not guarantee a stable displacement. Similarly, displacement can be stable if the lateral dimensions are much smaller than the longitudinal dimensions, even when very little vertical communication is present.

Concluding Remarks - The results presented in this and the preceding work show that both megascopic and macroscopic dispersivities have an Initial non-Fickian component where dispersivities are time-dependent. The duration of this non-Fickian flow depends on a number of factors including the correlation length, the magnitude of heterogeneity and the aspect ratios. Therefore, under certain conditions it is possible to have an initial channeling or fingering displacement even in laboratory cores. This initial non-Fickian period is followed by a transition period and then finally a Fickian displacement with constant dispersivities. As discussed here, some factors such as the 


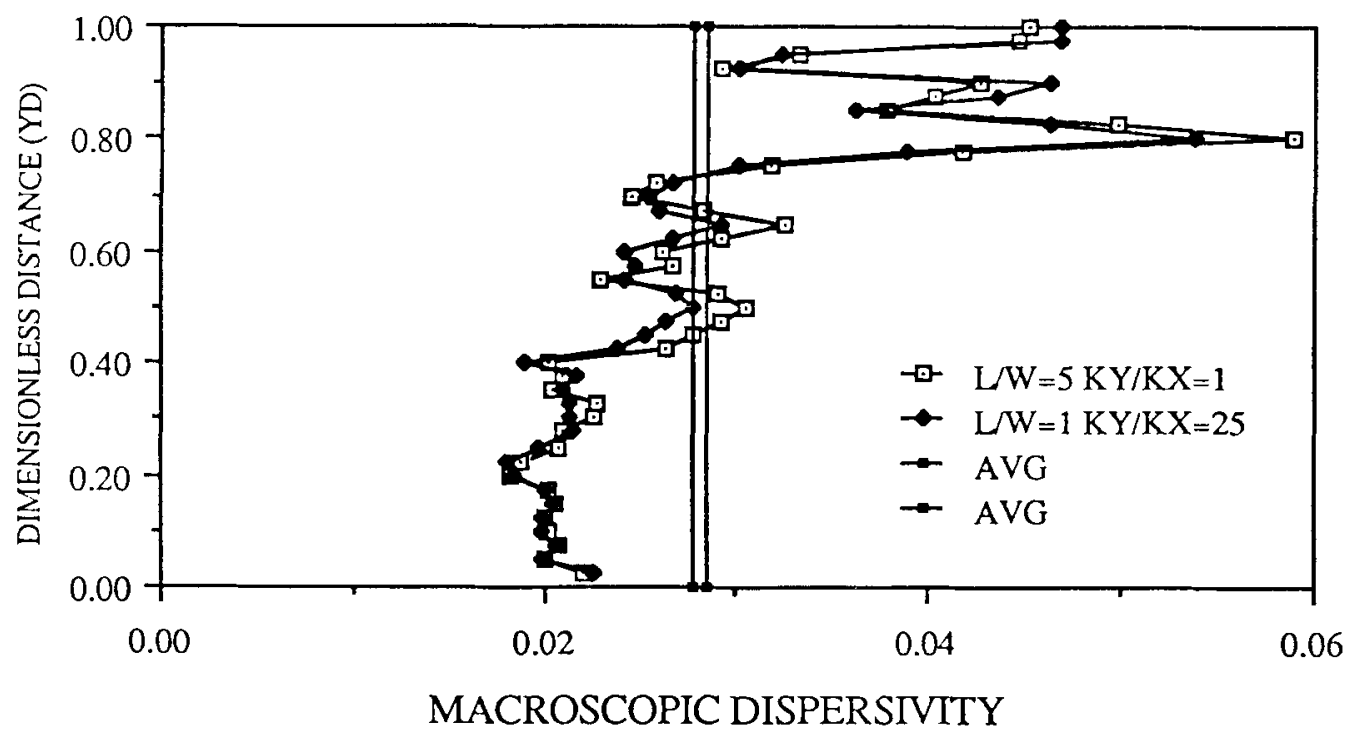

Figure II-25. Macroscopic dispersivities at a cross-section, $v_{D P}=0.6, x_{D}=0.5, R_{L}=5.0$. 


\begin{abstract}
presence of molecular diffusion can retard the growth of channels. However, if transport in permeable medium is modeled as a purely dispersive phenomenon, this early time behavior cannot be reproduced. It is possible to arrive at the same dispersivity (as measured at the outlet of a core) from a dispersive model and from a model with Fickian and non-Fickian components. However, the concentration distribution and the fluid recoveries will not be the same. The initial period of flux-controlled displacement, depending on its magnitude and duration, can have a significant influence on the recovery and the economics of the displacement process.

The results of this work demonstrate the drawbacks of using a purely dispersive model to describe transport through permeable medium. Such a model not only fails to give an accurate estimate of the magnitude of dispersivities but also does not give any information about the temporal and spatial variation of dispersivities.
\end{abstract}




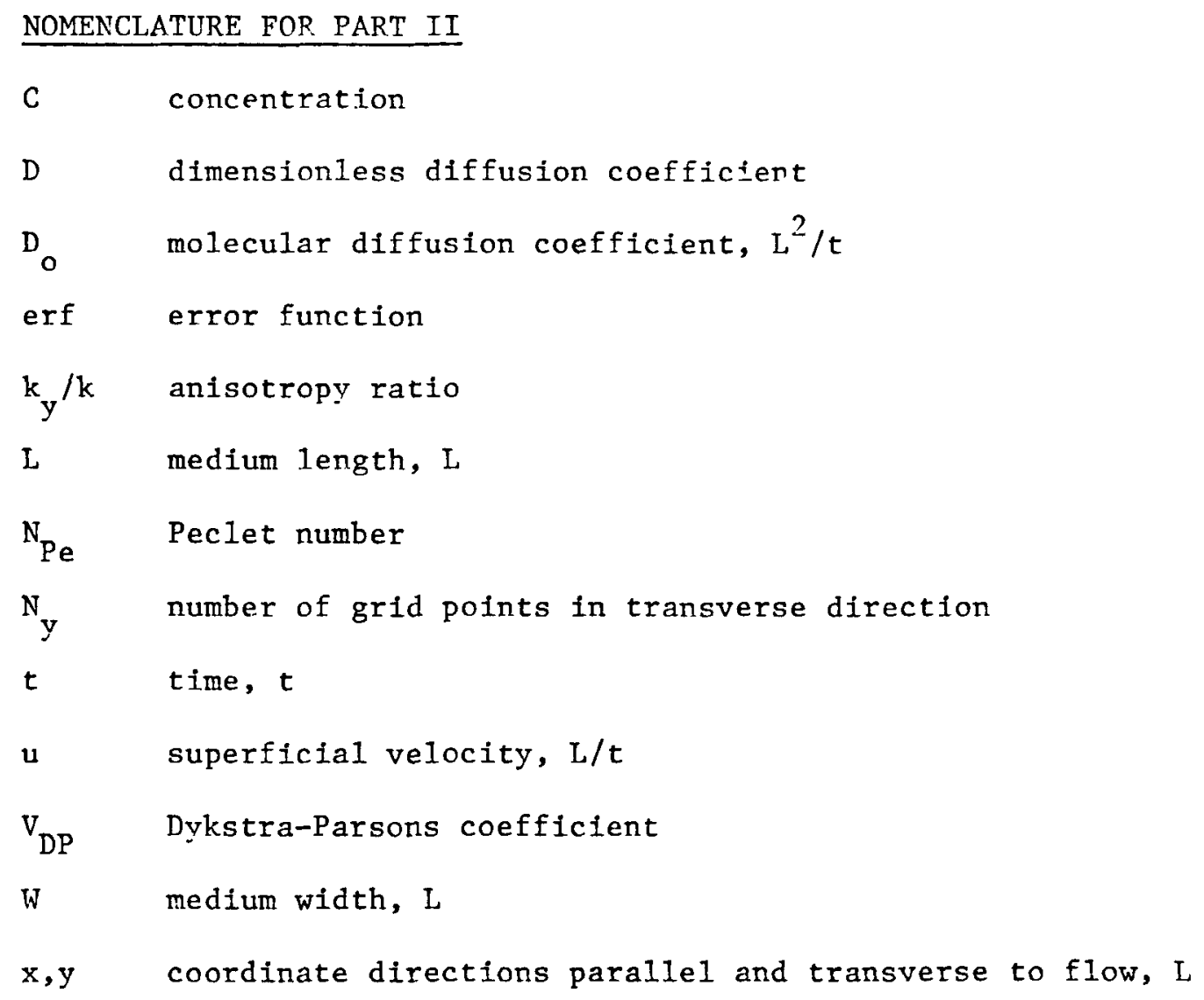

\section{$\underline{\text { Subscripts }}$}

D denotes a dimensionless quantity

\section{Greek}

$\alpha_{\text {MA }}$ macroscopic dispersivity, L

${ }^{\alpha} \mathrm{ME}$ megascopic dispersivity, L

$\lambda$ correlation length, $\mathrm{L}$ 
PART II REFERENCES

Arya, Atu1, "Dispersion and Reservoir Heterogeneity," Ph.D. Dissertation, the University of Texas in Austin, 1986.

Arya, A., Hewett, T. A., Larson, R. G., and Lake, L. W., "Dispersion and Reservoir Heterogeneity," SPE 14364 presented at the 60th Annual Technical Conference and Exhibition of the Society of Petroleum Engineers held in Las Vegas, Nevada, September 22-25, 1985.

Brigham, W. E., "Mixing Equation in Short Laboratory Cores," Soc. Pet. Eng. J., (Feb. 1974) 91-99, Trans., AIME, 257.

Dykstra, H. and Parsons, R. L., "The Prediction of Oil Recovery by Water Flood," API Secondary Recovery of Oil in the United States, (1950) 160.

Heller, J. P., "Observations of Mixing and Diffusion in Porous Media," Proc. Symp. Fundam. Transp. Phenom. Porous Media 2nd, 1, 1-26, 1971.

Lantz, R. B., "Qualitative Evaluation of Numerical Diffusion (Trucation Error), Soc. Pet. Eng. J. (Sept. 1971) 315-320; Trans., AIME, 251. 
III. DATA HANDLING

Any characterization procedure based on stochastic variables must be aware of the inherent limitations of statistics. There are several excellent and even traditional references of statistics, but few with the particular orientation of reservoir characterization. The work described in this section is intended to at least partially provide this direction. Once again, each subsection corresponds to projects outlined in the original proposal.

The first section deals with reconciling core and log-derived porosities through stochastic assignments. Next we discuss the effects of normality transformations on regression and the statistical properties of heterogeneity measures. The latter leads to a new measure of heterogeneity. After this we discuss the analysis and application of the turning bands method for generating stochastic line processes. Part $V$ returns to this briefly where it is applied to two-dimensional fields. 
RECONCIIING CORE AND LOG-DERIVED PROPERTIES

Introduction - Lack of adequate specification of reservoir properties remains a fundamental problem limiting the application of numerical simulators. Some of the necessary properties are obtained from both geophysical logs and core. In many cases values from these two sources are not in agreement. Within the overall goal of improving data handing, the objective of this section is to evaluate the causes of differences in porosity values obtained from cores and logs.

While many differences are because of problems in the measurement technique itself, we focus only on differences which may be attributed to the measurements on different scales. Core porosities can be considered "point" values and log-derived porosities volumetric averaged (smoothed) values. The degree to which core (assumed actual) porosity correlates with and are well represented by log-derived (averaged) porosity should be a function of the smoothing bias introduced by the logging technique, the heterogeneity of the system (rock sequence), and the sampling density.

Premises - Our starting premise is that the variability observed in a set of measurements (through a rock sequence) can be a direct function of the scale (volume) of the measurement itself. For a given heterogeneity, we expect less variance in a data set as the volume sampled by the measurement technique increases. Thus, for any given heterogeneous rock sequence, core porosity should show more variance about the mean porosity value, than log-derived porosity. As the volume measured by a geophysical logging device decreases and 
approaches that measured by cores, variance in the recorded data sets will approach one another, and correlation between the recorded porosity values for the two measurement techniques will improve. For any specific heterogeneity Fig. III-1 illustrates expected correlation and variance relationships.

In addition, we expect the degree of correlation between porosity values for the two measurement techniques to be some function of the actual heterogeneity existing in the rock sequence. In the limiting and hypothetical case of a homogeneous rock sequence, actual (core) porosity at any depth, constant by definition, will obviously correlate perfectly with averaged (log-derived) porosity values for the same depth. As heterogeneity increases, the degree of correlation for any one averaging measurement technique should decrease. Further, there should be a family of curves representing correlation obtainable for a specific averaging measurement technique. The expected relationship between heterogeneity and correlation is illustrated in Fig. III-2.

For any given heterogeneity, the degree to which core and log-derived porosity values will correlate should be some function of the frequency of core measurements. The higher the density of core measurements, the more representative the recorded data should be of the true variability, and the higher the expected correlation with an averaged measurement obtained by a geophysical logging technique. We also expect that greater sampling density is required to obtain the same degree of correlation or precision in determination of porosity distribution as heterogeneity increases. These expected relationships are illustrated in Fig. III-3. 


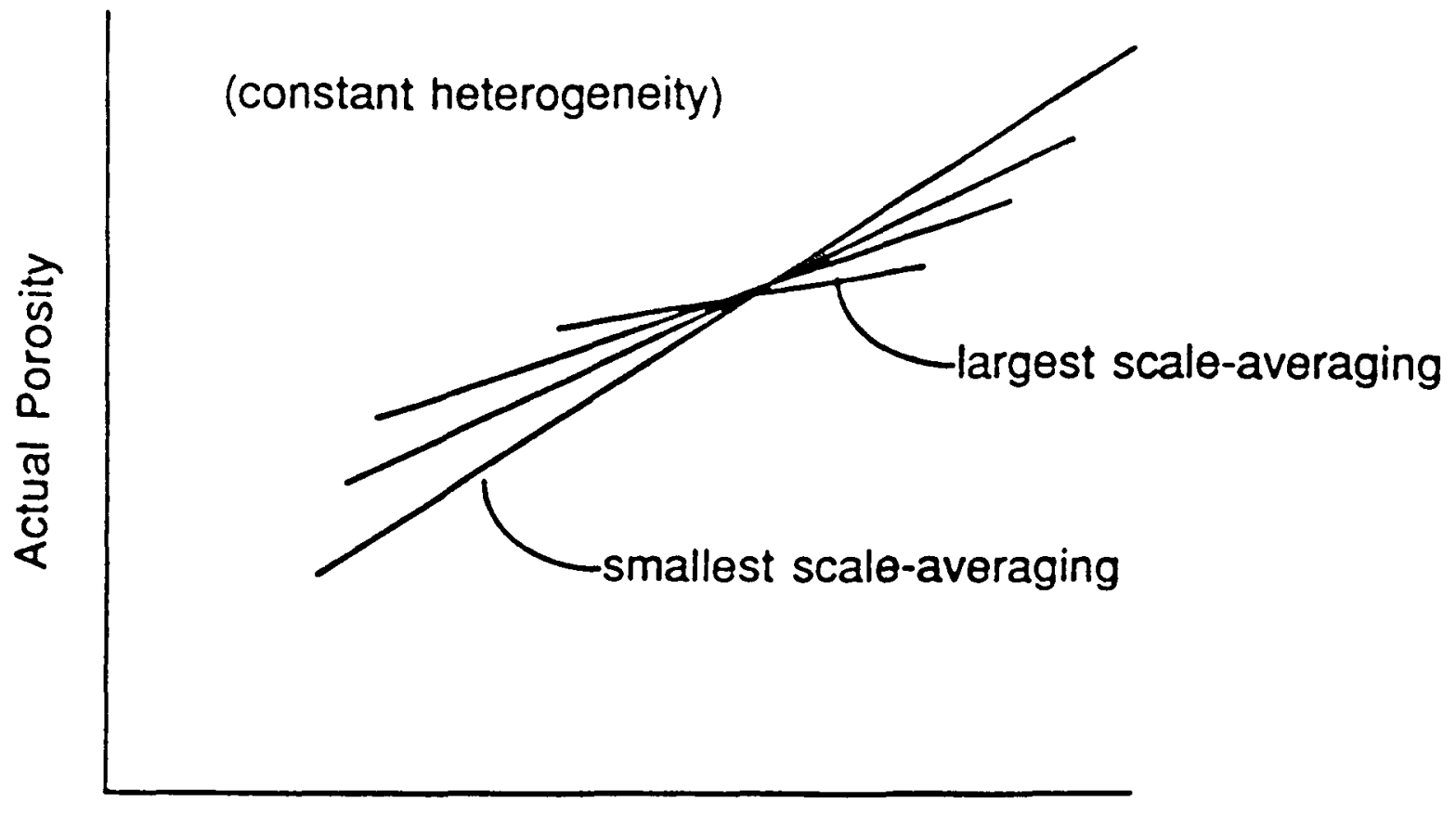

Cumulative Frequency

Figure III-1. Decreased variance observed with increased scaleaveraging measurement technique.

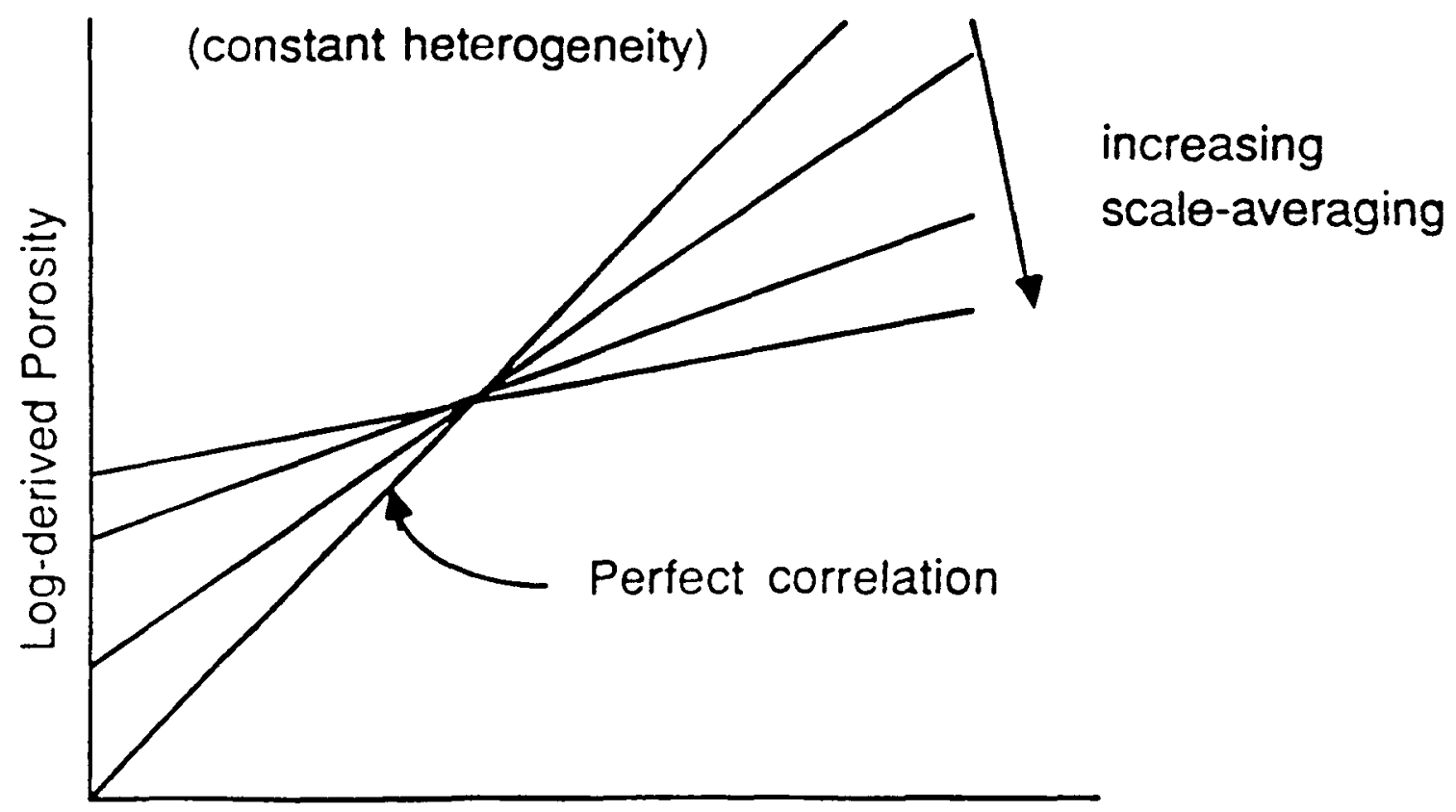

Actual Porosity

Figures III-la. Expected correlations as a function of scaleaveraging size (any given heterogeneity). 


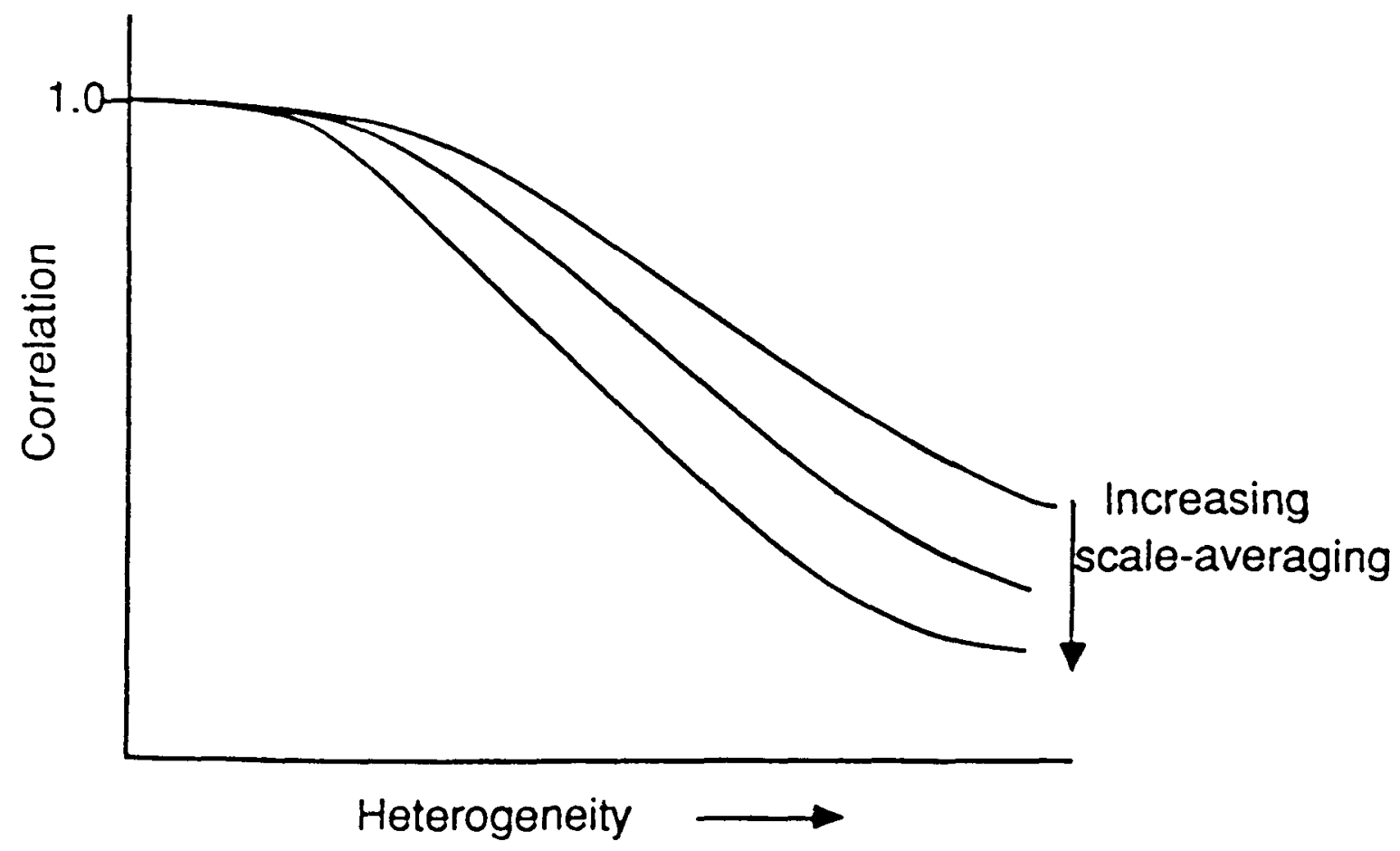

Figure III-2. Family of curves depicting correlation for various scale-averaging techniques for any heterogeneity.

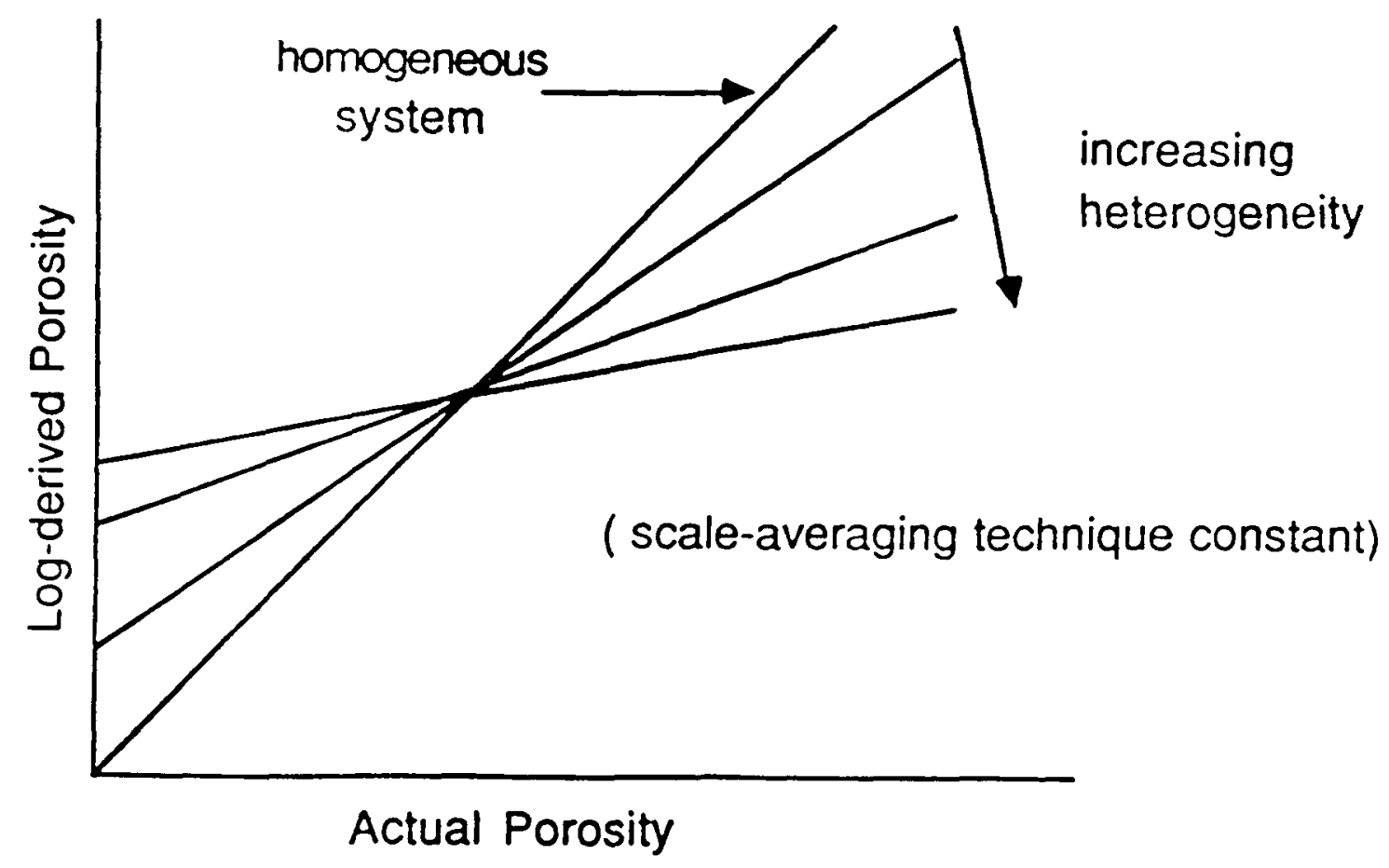

Figure III2a. Expected correlations as a function of heterogeneity (constant scale-averaging technique). 


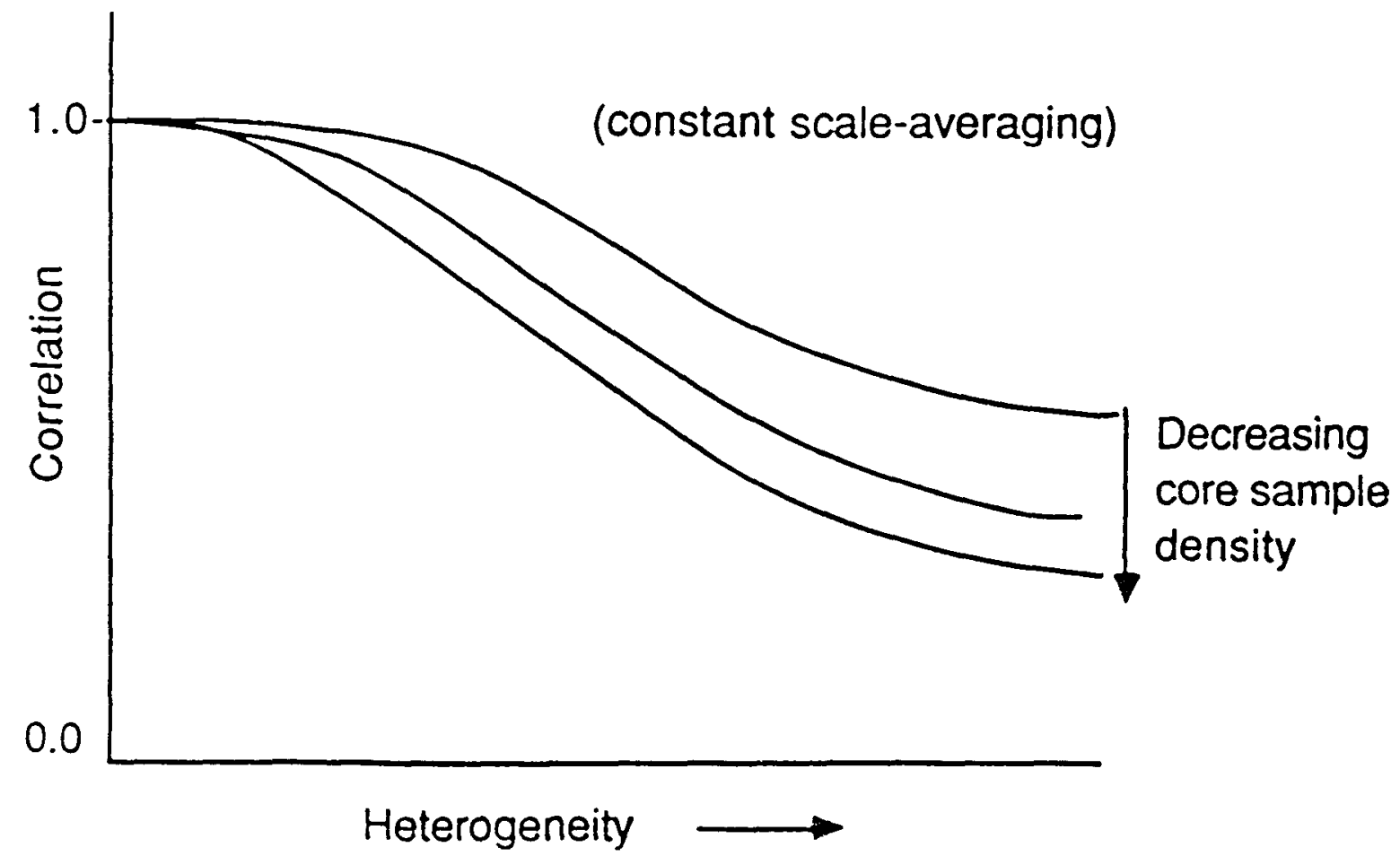

Figure III-3. Family of Curves Depicting Correlation for Various Core Sample Density as a function of Heterogeneity

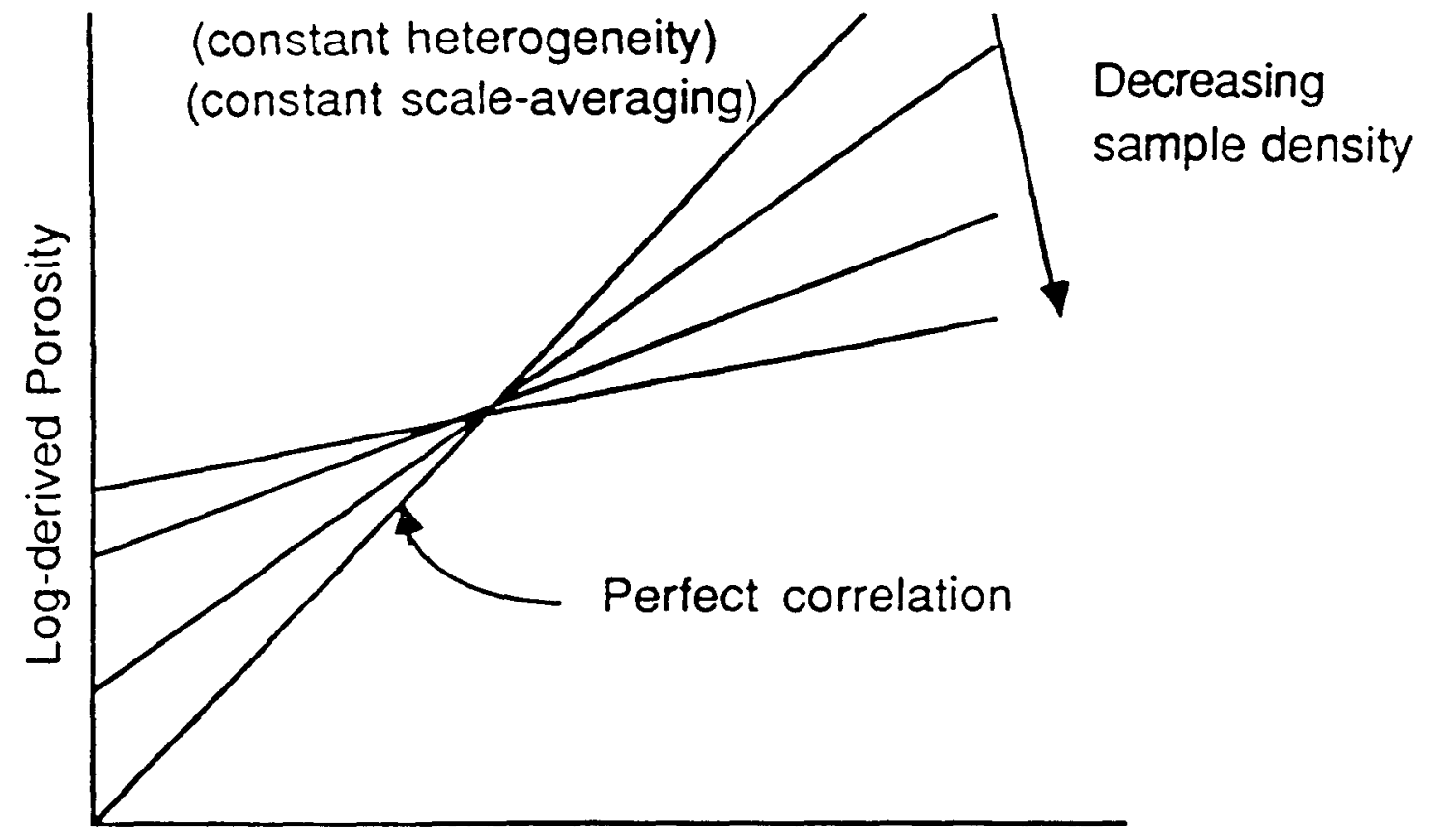

Actual Porosity

Figure III3a. Expected Correlation as a Function of Sample Density for any Specific Heterogeneity and Specific Scaleaveraging Technique 
Approach - The initial approach taken in investigating and quantifying these hypotheses has been to develop a synthetic porosity profile (porosity distribution vs depth) of a specific heterogeneity (in this case variance about the mean). The synthetic profile represents "truth", and the "goodness of measurement" of this "truth" is tested statistically for different scale-averaging (log-type smoothing) functions and sampling frequencies. The profile is modified to simulate various heterogeneities, and the effect evaluated. Families of curves for different heterogeneities, scale-averaging schemes, and sample density measurements are being generated.

The resultant family of curves should be useful in defining heterogeneity from knowledge of the measurement technique and correlation obtained from recorded core and $\log$ data. It may be possible to obtain a better measure of the "true" heterogeneity based on observed geophysical log-derived porosity data. For a given heterogeneity and averaging scheme, it may be possible to normalize the log-derived porosities to "actual" porosities, the greatest benefit being the ability to produce a porosity distribution which more closely represents the actual distribution. In addition, this work may suggest an optimal sampling frequency and smoothing function relative to the expected heterogeneity or degree of characterization desired.

Following these synthetic model studies, core and $\log$ data from a location, such as the EI Dorado Field, will be used to investigate the application of the derived techniques. 
OPTIMIZATION OF REGRESSION-BASED POROSITY-PERMEABILITY PREDICTIONS

Introduction - Porosity-permeability relationships, developed by regressing on data from cored wells, are used to predict reservoir permeability from well logs for uncored wells. Several field studies (Tootle, 1979; Wendt et a1., 1985; Wilson and Hensel, 1978; Brown and Husseini, 1977; Boyer, 1984; Matiisen and Atwater, 1984) that discuss the development of the predictive relations indicate widely variable success with standard regression procedures.

Undoubtedly part of the problem in successfully predicting permeability from porosity is due to the inexact nature of the porosity-permeability relationship. For example, Levorsen (1969) and Pottier et al. (1964) Indicate that permeability is not a function of porosity alone. Any procedure using exclusively porosity to determine permeability is limited in success by the variability of grain size and other quantities not accounted for by porosity. Furthermore, the porosity-permeability relationship for a formation is not known prior to taking core data; we have only empirical observations to guide us. Nonetheless, several studies (e.g. Wendt et al., 1985) show that porosity may be a good indicator of permeability for particular fields. The issue is how to exploit this association to the fullest to obtain the best estimate possible for permeability. A review of the literature detailing field studies with permeability prediction shows features common to many of these reports:

1) Regression is used to relate core permeability $k$ to core porosity $\phi ;$ 
2) A linear model of the form $\log (k)=a \phi+b$ is assumed, where a and $b$ are the regression parameters;

3) The permeability estimate $(\hat{\mathrm{k}})$ is obtained using

$$
\hat{k}=(10)^{a \phi+b}
$$

for a measured value of porosity $\phi$; and

4) It is not indicated how the predictions are to be used. That is, whether they will be combined (to give an average permeability for example) or if they will be treated separately.

In general, the above features may lead to inferior results. The porosity-permeability relation may not be used to its fullest in points (1) and (2) because the linear model does not capture any nonlinearity in the $\phi-\log (k)$ association. Step (3) creates a permeability estimate which typically is pessimistic because exponentiation is a nonlinear transformation. Finally, item (4) is of concern since regression is a minimization procedure; it is important to know if the quantities being minimized are compatible with the ultimate objective.

Many of these issues can be addressed by appropriately transforming the core data prior to regression. By means of examples, we show that errors in permeability estimation can be reduced substantially by carefully using transformations. A judicious choice of transformation may also eliminate the need for weighted least squares and other, more complicated, regression techniques. 
Theory of Correlation and Regression - The theory of correlation and regression is well documented in such books as Kendall and Stuart (1969), Weisberg (1980), and Hald (1952). We address the problem in the context of permeability prediction using porosity, although what is discussed has applications in other relationships. Assume that some function of porosity, $g(\phi)$, and a function of permeability, $f(k)$, are to be used in determining the $\phi--k$ relationship. For example, when one sees $\phi$ versus $\log (k)$ plots of data $g$ is the identity function and $f$ is the logarithmic function. The precise forms of these functions is discussed below. Furthermore, we assume that the permeability predictions are to be used individually -- to predict an injection profile, for example -- or are to be used in linear combinations, such as in an arithmetic average to predict arithmetic average permeability. The reason for this assumption is also discussed below. We seek a curve which relates $f(k)$ and $g(\phi)$. The curve will be then used to give estimates of $f(k)$ when $\phi$ is known.

We begin by noting several features about this situation:

1) Both porosity and permeability are random variables, and hence $g(\phi)$ and $f(k)$ are random variables.

2) The form (1. e. equation) of the $\phi-k$ relation is not known prior to taking data, and

3) The $\phi-k$ relation may change character as the rock fabric changes. Item (1) implies that the joint probability density function (j.p.d.f.) of the variables $g(\phi)$ and $f(k)$ should be considered when establishing the $\phi-k$ association. The j.p.d.f. captures all there is to know about the statistical behavior of $\phi, k$, and the $\phi-k$ 
relationship. It shows, among other things, the variability of $f(k)$ when $g(\phi)$ is fixed and vice versa.

Feature (2) indicates that a problem may arise if regression is used to best fit an arbitrary curve to the data. The form of the curve is unknown and previous experience, item (3), does not necessarily help. Hence, fitting a polynomial or some other curve to the data may not optimally capture the relationship of $\phi$ and $k$.

Significance tests for the regression coefficients may be useful in determining whether the regression has captured the porosity-permeability association well. However, such tests are helpful only when the permeability data $f(k)$ are nearly normally distributed. Another advantage when $f(k)$ is approximately normal is that the regression curve obtained by least squares may become the best curve possible under the circumstances. That is, the curve may give the closest estimate to the correct value of $f(k)$ when $f(k)$ is approximately normally distributed.

Even if $f(k)$ is normally distributed, the form of the $\phi-k$ relation is still in question. For example, suppose the $g(\phi)-f(k)$ relationship is quadratic but we have chosen a linear model. Then, even if we obtain the best linear fit possible, errors arise because the model is not appropriate for the relationship. However, if the j.p.d.f. for the $g(\phi)--f(k)$ relationship is a bivariate normal distribution, the curve relating $g(\phi)$ and $f(k)$ is a straight line (Hald, 1952).

For $g(\phi)$ and $f(k)$ to be bivariate norma11y distributed (b.n.d.), it is a necessary condition that both $f(k)$ and $g(\phi)$ are approximately 
normally distributed. Once we know that the j.p.d.f. of $g(\phi)$ and $f(k)$ is approximately bivariate normal, a simple regression of the form

$$
f(k)=a \cdot g(\phi)+b
$$

captures optimally the $\phi-k$ relation. A similar situation occurs when more than one variable is used to predict permeability; a linear regression model optimally captures the relationship for variables that are multivariate normally distributed.

Another advantage of $g(\phi)$ and $f(k)$ being b.n.d. is that estimator bias may be accounted for. Consider, for example, the case where $g(\phi)=\phi, f(k)=\ln (k)$, and the regression parameters $a$ and $b$ in $f(k)=a g(\phi)+b$ have been determined. In the regression procedure, the line $y=a \phi+b$ is established so that the spread of points above the line is roughly the same as the spread of points below the line, Fig. III-4 (Fig. III-5 referred to on page 76). For any given value of $\phi$, call the spread of points above the line $\delta_{+} y$ and the spread of points below the line $\delta_{-} y$. What happens to the spread when the line $a \phi+b$ is used to predict a permeability? The line $y=a \phi+b$ gives the estimate of $\ln (k)$ with possible error of $\delta_{+} y$ too high or $\delta_{-} y$ too low. Exponentiating the prediction gives $k=\exp \{y\}$ which may be too high by

$$
\delta_{+} \hat{k}=\exp \left\{y+\delta_{+} y\right\}-\exp \{y\}
$$

or too low by

$$
\delta_{-} \hat{k}=\exp \{y\}-\exp \left\{y-\delta_{-} y\right\}
$$




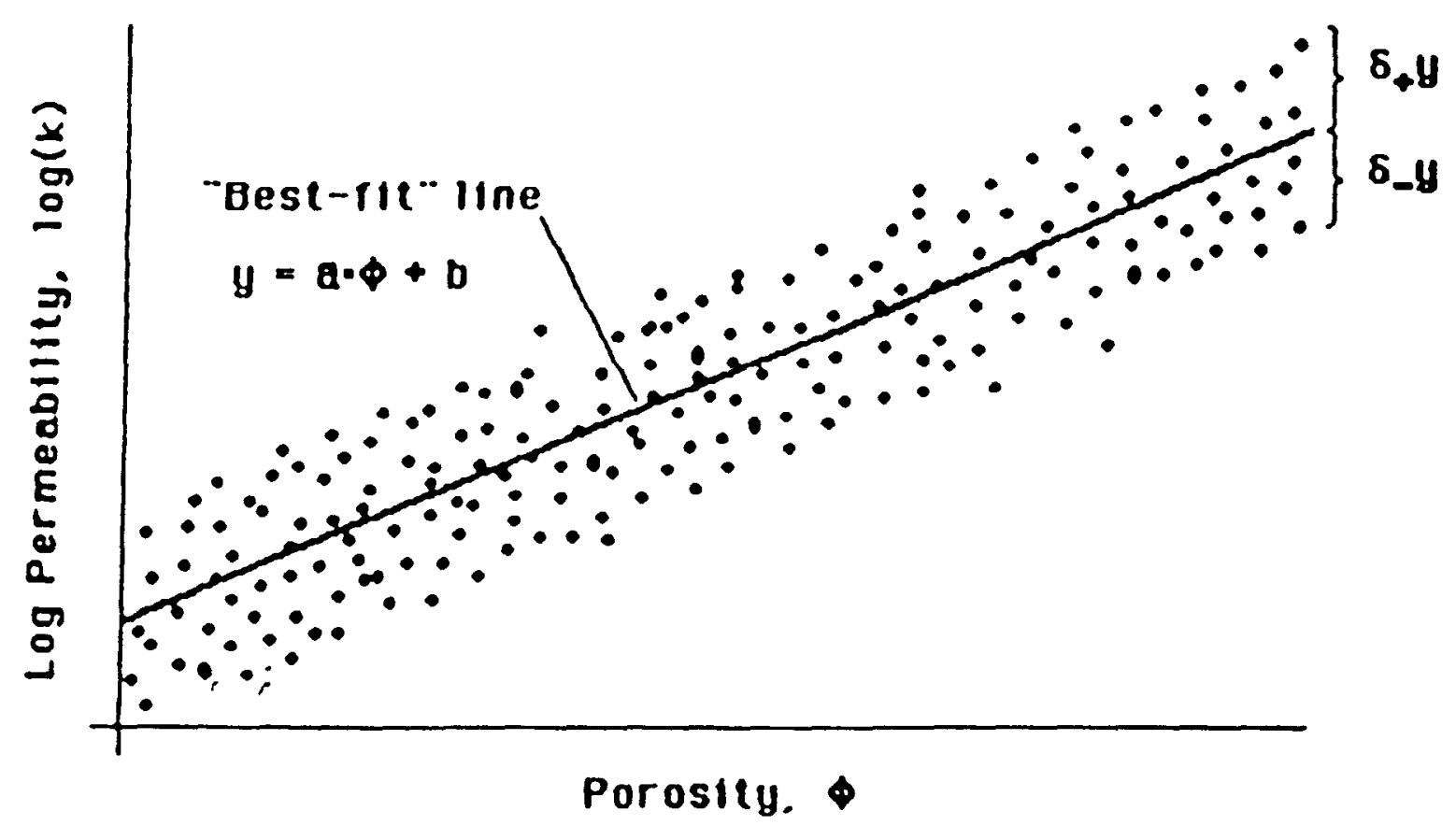

Figure III-4. Hypothetical porosity-permeability plot.

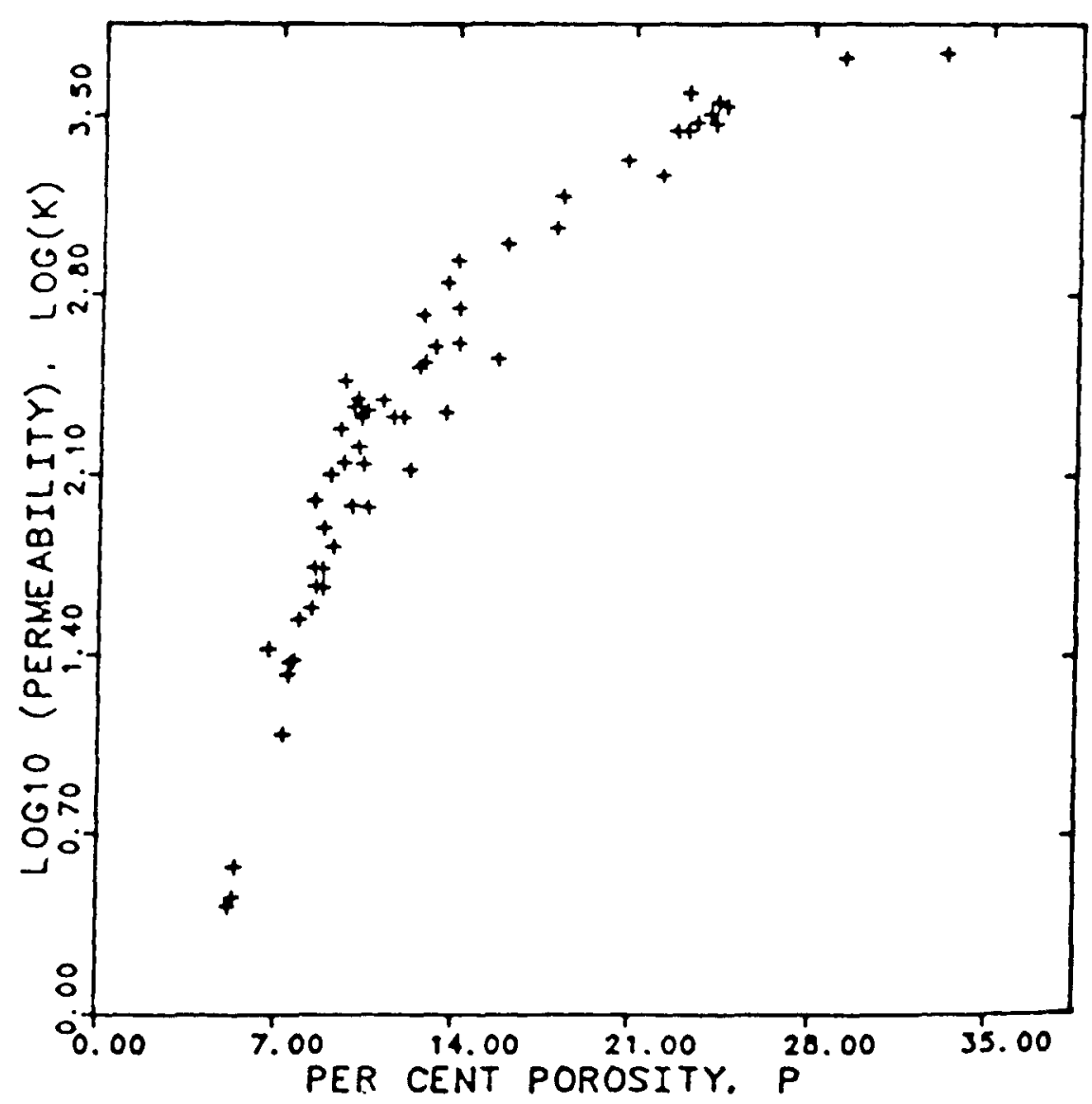

Figure III-5. Porosity and permeability data for Fontainebleau Sandstone. (after Jacquin, 1964). 
From the definition of $\hat{k}$, Eqs. (III-1) and (III-2) become

$$
\delta_{+} \hat{k}=\hat{k} \cdot\left\{\exp \left[\delta_{+} y\right]-1\right\}
$$

and

$$
\delta_{-} \hat{k}=\hat{k} \cdot\left\{1-\exp \left[-\delta_{-} y\right]\right\}
$$

Because the exponential function is nonlinear, the equal quantities $\delta_{+} \mathrm{y}$ and $\delta_{-} \mathrm{y}$ give unequal amounts $\delta_{+} \hat{\mathrm{k}}$ and $\delta_{-} \hat{\mathrm{k}}$. Suppose $\delta_{+} \mathrm{y}=$ $\delta_{-} y=1.2$ (about $1 / 3$ of a decade). $\delta_{+} \hat{k}=2.3 \hat{k}$ while $\delta_{-} k=0.7 \hat{k}$. So the regression line gives an estimate $\hat{k}$ that $i s$, on average, biased. Below we show how to address this problem of bias when $g(\phi)$ and $f(k)$ are b.n.d.

In summary, if $f(k)$ is approximately normally distributed (a.n.d.), several advantages are obtained in the regression. If both $g(\phi)$ and $f(k)$ are b.n.d., then a simple linear regression captures the $\phi--k$ relationship and bias may be compensated for. How to choose $f$ and $g$ is the next consideration.

Power Transformations - A data transform $h(\cdot)$ applied to a set of data $\left\{x_{i}\right\}$ produces a data set $\left\{y_{i}\right\}$, where

$$
y_{1}=h\left(x_{1}\right)
$$

Data transformations are common in the statistical literature (see, for example, Hoyle, 1973, or Kruskal, 1968). Data are usually transformed to achieve one of three objectives: 
1) Additivity - the primary effects influencing the data combine linearly;

2) Homogeneity - the variability of the data is constant as parameters are changed; and

3) Normality - the transformed data become a.n.d.

often, transformation to satisfy one of the objectives leads to satisfaction of the other objectives. We seek transformations $f$ and $g$ to give an approximately bivariate normal distributed data set.

A useful transformation is the power transformation. It has the form $h\left(x_{i}\right)=\left(x_{i}\right)^{q}$ for some exponent $q$. Tukey (1957) recogntzed that power transformations have many common features. He treated power transformations as a family:

$$
h\left(x_{i}\right)= \begin{cases}\left(x_{i}+c\right)^{q}, & q \neq 0 \\ \ln \left(x_{i}+c\right), & q=0\end{cases}
$$

where $c$ is a constant. The choice of function for the case $q=0$ may be surprising until a slightly different family is considered

$$
h^{\prime}\left(x_{i}\right)= \begin{cases}\frac{\left(x_{i}+c\right)^{q}-1}{q}, & q \neq 0 \\ \ln \left(x_{1}+c\right) & , q=0\end{cases}
$$

Both $h$ and $h^{\prime}$ have the same p.d.f. since only a linear rescaling is involved. Furthermore, $\lim \left[(z)^{q}-1\right] / q=\ln (z)$. $q \rightarrow 0$

Despite its utility, the approach of treating power transformations as a family appears to be new to the petrophysical literature. Previous statistical work has concentrated primarily on 
the members $q=0$ (logarithmic transform) and $q=1$ (identity transform). Fowever, Langeland and Flotre (1084) discuss the use of power transformations for porosity-cation exchange capacjty relationships and Jensen et a1. (1985) have shown that $(k)^{p}$ is a.r.d. for some $p,-1$ $\leqq p \leqq+1$. Furthermore, our experience is that usually a value of $q$ may be found such that $(\phi)^{9}$ is a.n.d.

By transforming porosity and permeability, the j.p.d.f. we are concerned with changes. The porosity-permeability relationship does not change; instead we create "new" random variables, $(\phi)^{q}$ and $(k)^{p}$, such that the j.p.d.f. of these variables is close to bivariate normal. A simple linear regression of the form $(k)^{p}=a \cdot(\phi)^{q}+b$ then expresses the $\phi-k$ relation optimally.

Several methods exist to estimate the exponents $p$ and $q$. Gnanadesikan (1977) reviews several of these methods. We have had good results using the method of Emerson and Stoto (1982), which estimates an exponent based on symmetrizing the p.d.f. While symmetrical and normal p.d.f.'s are not necessarily the same, the method has worked well thus far. Another method is to try several values of exponent on a data set and determine which exponent value gives the straightest line on a normal probability plot. This approach may be the easiest to implement with existing computer analysis routines.

While values $p$ and $q$ may be found such that both $(k)^{p}$ and $(\phi)^{q}$ are a.n.d., this is not a sufficient condition for $(k)^{p}$ and $(\phi)^{q}$ to be b.n.d. Further tests exist to determine if variables are b.n.d. Hald (1952) describes one test and Gnanadesikan (1977) devotes a section to discussing various tests and examples for hypothetical data sets. Our 
experience indicates that transformation to symmetry is usually helpful regardless of the resulting joint distribution. The results we show based on the assumption of bivariate normality appear to be relatively insensitive to this assumption provided that symmetry is obtained.

Correction for Estimator Bias - Here we address the problem of how to predict permeability $k$ given that we have an estimate $y=(k)^{p}$. We show that $(y)^{1 / p}$ does not necessarily give the best possible estinate for $k$. However, a correction term can be calculated to resolve the problem.

Suppose that $(k)^{p}$ and $(\phi)^{q}$ are $b \cdot n \cdot d$. for some values of $p$ and $q$. Define

$$
x=\frac{(k)^{p}-1}{p}
$$

The variable $x$ is normally distributed with mean $\mu_{x}$ and variance $\sigma_{x}^{2}$. At a specified porosity $\phi$, the variable $x$ is normally distributed with mean $\mu=a \cdot(\phi)^{q}+b$ and variance $\sigma^{2}=\sigma_{x}^{2}\left(1-\rho^{2}\right)$, where $a$ and $b$ are the regression parameters of the $x$ versus $(\phi)^{q}$ data and $\rho$ is the coefficient of correlation (Hald, 1952).

For a given value of $\phi$, we seek a permeability estimate, $\hat{k}$, based on the estimate $x=\mu=a \cdot(\phi)^{q}+b$ and Eq. (III-3). The estimate $k$ is given by

$$
\hat{\mathrm{k}}=\mathrm{E}\{\mathrm{k} \mid \phi\}
$$


where $\mathrm{E}$ is the expectation operator. Using Eq. (III-3), Eq. (III-4) becomes

$$
\hat{\mathrm{k}}=\mathrm{E}\left\{(\mathrm{px}+1)^{1 / \mathrm{p}} \mid \phi\right\}
$$

The exact solution of Eq. (III-5) is shown in Jensen (1985) to be

$$
\hat{k}=\frac{(p \sigma)}{\sqrt{2 \pi}}^{1 / p}[\Phi(v)]^{-1} \exp \left(-\frac{1}{4} v^{2}\right) \Gamma\left(\frac{1}{p}+1\right) U\left(\frac{1}{p}+\frac{1}{2},-v\right)
$$

where

$$
\mathrm{v}=\frac{1+\mathrm{p} \mu}{\mathrm{p} \sigma}
$$

and $\Phi, \Gamma$, and $U$ are functions which are defined in Abramowitz and Stegun (1972). When $p=0$ (the case of log-normal permeability), Eq. (III-6) gives

$$
\hat{\mathrm{k}}=\exp \left\{\mu+\frac{1}{2} \sigma^{2}\right\}
$$

Note that if $|\rho|=1.0$ (perfect correlation), $\sigma^{2}=0$ and no bias occurs. Otherwise, if $|\rho|<1.0$ and $\sigma_{x}^{2}$ is 1arge, a substantial correction arises. For the log-normal case, the correction is to multiply $\exp \left[a \cdot(\phi)^{q}+b\right]$ by $\exp \left(0.5 \sigma^{2}\right)$.

Equation (III-6) has an intimidating look, so we present by example an alternative method of computing $k$ for the case where $m=$ $1 / \mathrm{p}$ is an integer and $\sigma_{\mathrm{x}} / \mu_{\mathrm{x}} \leqq 0.43$. Suppose $\mathrm{m}=2$. Equation (III-5) may be expressed as

$$
\hat{\mathrm{k}}=E\left\{\left(\frac{1}{2} \mathrm{x}+1\right)^{2} \mid \phi\right\}
$$

or 


$$
\hat{\mathrm{k}}=\left(\frac{1}{2} \hat{\mathrm{x}}+1\right)^{2}+\frac{1}{4} \sigma^{2} \text {, }
$$

where we have used the fact that $\mathrm{E}\left(\mathrm{x}^{2} \mid \phi\right)=\mu^{2}+\sigma^{2}$. Using Eq. (III-3) for $\mathrm{p}=\frac{1}{2}$ and letting $\mathrm{y}=(\mathrm{k})^{\frac{1}{2}}$, Eq. (8) becomes

$$
\hat{\mathrm{k}}=\hat{(\mathrm{y})^{2}}+\frac{1}{4} \sigma^{2} .
$$

Note that $\hat{y}$ is the estimate of $(k)^{\frac{1}{2}}$ from the regression line $0.5 a(\phi)^{q}$ $+0.5 b+1$. The correction term in Eq. (III-9), arising from the nonlinearity of the square function, depends both on the degree of correlation $\rho$ and the variability of the data $\sigma_{\mathrm{x}}^{2}$. If we denote the variance of $(k)^{\frac{1}{2}}$ as $\sigma_{y}^{2}$, Eq. (III-9) becomes

$$
\hat{\mathrm{k}}=(\hat{\mathrm{y}})^{2}+\sigma_{\mathrm{y}}^{2}\left(1-\rho^{2}\right)
$$

The Influence of Prediction Usage - The method of least squares originates from the Gauss-Markov theorem (Liebelt, 1967). This theorem states how an optimal estimate may be calculated for a specific problem once certain assumptions are satisfied. One requirement imposed by the Gauss-Markov theorem is that the estimates are combined in a linear manner. This requirement affects how the permeability estimates may be used.

For example, suppose we wish to estimate average reservoir permeability $\bar{k}$ using $n$ estimates $\left\{k_{1}, i=1 \ldots n\right\}$ from the $\phi-k$ relation. There are several ways to calculate average permeability. The arithmetic average, the geometric average, and the harmonic average are three possibilities. The arithmetic average combines each prediction linearly: 


$$
\overline{\mathrm{k}}=\frac{1}{\mathrm{n}} \sum_{\mathbf{i}} \hat{\mathrm{k}}_{\mathrm{i}} .
$$

But the geometric and harmonic averages combine the estimates in a non-1inear manner:

$$
\left.\bar{k}=\underset{i}{\left(\pi \hat{k}_{i}\right.}\right)^{1 / n}
$$

and

$$
(\bar{k})^{-1}=\sum_{i}\left(\hat{k}_{i}\right)^{-1}
$$

respectively. Just as in the preceding discussion, nonlinearity causes a bias in the estimates of the harmonic and geometric averages. Consequently, there is no basis for presuming that linear regression gives us the optimal estimate of average permeability if we combine the predictions in a nonlinear manner.

Generally speaking, the problem of obtaining optimal estimates of quantities which are nonlinear functions of other estimates is a difficult one. When such estimates come from a regression based on b.n.d. variables, a correction can be made, just as in the case for estimator bias previously discussed. The calculations are quite involved, however. We present an example below to demonstrate how nonlinear averaging may influence estimates for average reservoir permeability.

Examples - We begin by analyzing a small data set using the power transformation scheme just discussed. A good fit to the transformed data is obtained using a simple regression model. Two larger data sets are then studied. The first demonstrates how data may behave with different transformations and how these transformations can 
influence the prediction of permeability. The last data set analysis shows that ignoring estimator bias can lead to predictions that are 23 percent tco low.

Case 1 - Figure III-5 is a porosity-1ng permeability plot for sixty core plug data as reported by Jacquin (1964). We have chosen this data set because of the strong $\phi-k$ association. Clearly, the relationship is nonlinear and Jacquin proposes the equation $k=$ $c \cdot(\phi)^{4.5}$ as being a good representation of the relationship, although he does not specify a value for the constant $c$. The coefficient of correlation -- a measure of the linear relationship between the $x$ - and $y$-axis quantities ( $\phi$ and $\log (k)$ in this case) -- is 0.90 for Fig. III -5 .

Our approach is to determine the values of exponents $p$ and $q$ such that $(k)^{\mathrm{P}}$ and $(\phi)^{\mathrm{q}}$ are each approximately normally distributed. A glance at the histograms of porosity and log permeability, Figs. III- 6 and III-7 respectively, indicate that no exponents $p$ and $q$ exist which will transform either $(k)^{p}$ or $(\phi)^{q}$ to approximate normality; both distributions are distinctly bimodal. It appears that there may be two populations represented by these data. Only eight or ten points appear to belong to the population represented by the cluster of points near $\phi=24$ percent and $\log (k)=3.5$, however. To separate the data and obtain two $\phi-k$ relationships would probably not be useful with so few points in the higher porosity and permeability population. Consequently, we computed values of $p$ and $q$, based on the method of Emerson and Stoto (1982), to symmetrize (as much as possible) the distributions of permeability and porosity. The values $p=0.05$ and $q$ 


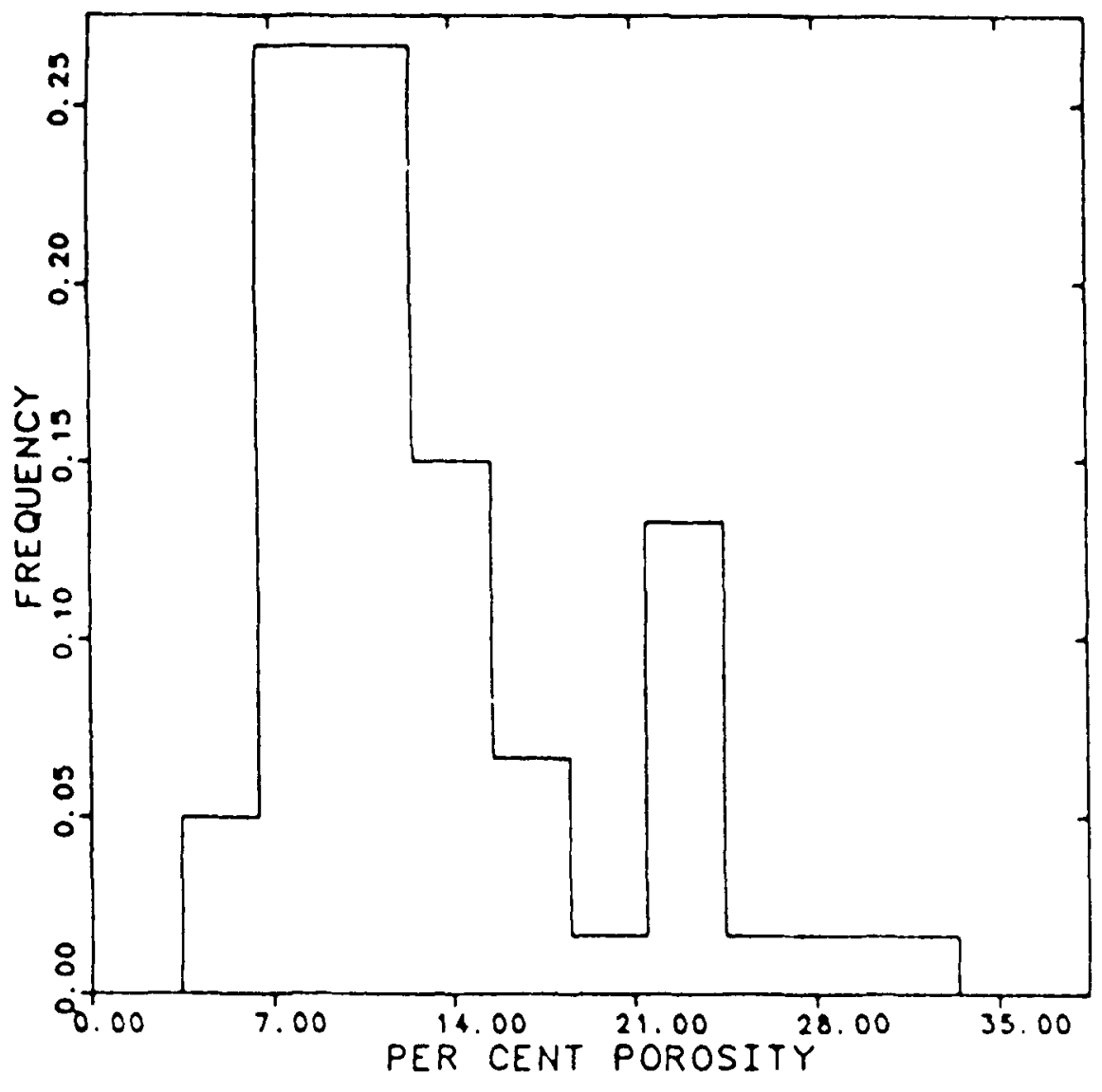

Figure III-6. Fontalnebleau Sandstone porosity Histogram

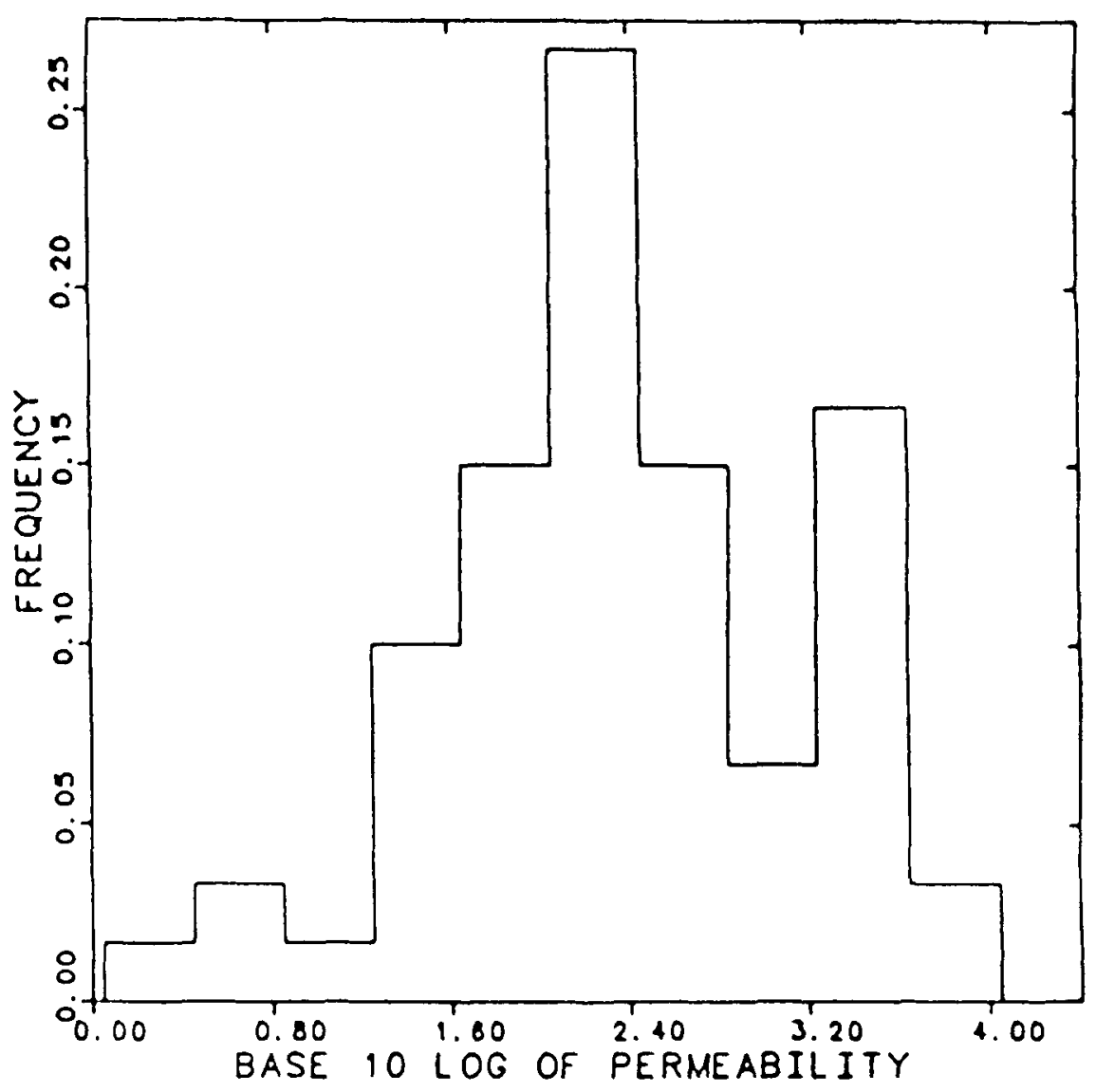

Figure III-7. Fontainebleau Sandstone premerablitty his togram. 
$=-0.6$ were obtained. We chose to use the transformations $\log (k)$ and $(\phi)^{-0.6}$. Figure III-8 is a plot of the data using these transformations and the coefficient of correlation is -0.98 . A simple Inear model, $\log (k)=a \cdot(\phi)^{-0.6}+b$, where $a=-13.5$ and $b=5.51$, fits the transformed data set.

Figure III-9 shows the data and two lines: Jacquin's proposed curve (A) and the curve based on a least-squares fit of Fig. III-8 (B). The value of the constant $c$ chosen for Jacquin's curve, 0.00319 , was made based on the position of the curve in his Fig. 10. Line B fits the data better at the low- and high-porosity regions. Without a statistical motivation for selecting $q=-0.6$, relating $\log (k)$ and $(\phi)^{-0.6}$ is not an obvious choice.

Case 2 - We turn now to the analysis of a larger (285 points from 16 wells) data set from the Admire sand in the E1 Dorado field in Kansas. The data set consists of core plug porosities and permeabilities from this shallow delta sand. Jordan and Tillman (1982) have reported an in-depth geologic and petrophysical analysis of the Chesney lease from which many of the data were extracted. See Jensen (1985) for more details concerning this data set.

An analysis of these data indicates that estimates for $p$ and $q$ are 0.50 and 5.5, respectively. The effects of transforming porosity and permeability are shown in Figs. III-10 through III-12, a series of porosity-permeability plots.

Figure III-10, the $\phi$ versus $\log (k)$ plot, is much like the plots in other field studies (e.g. Wendt et al., 1985) showing the porosity-permeability relationship. The variability of permeability 


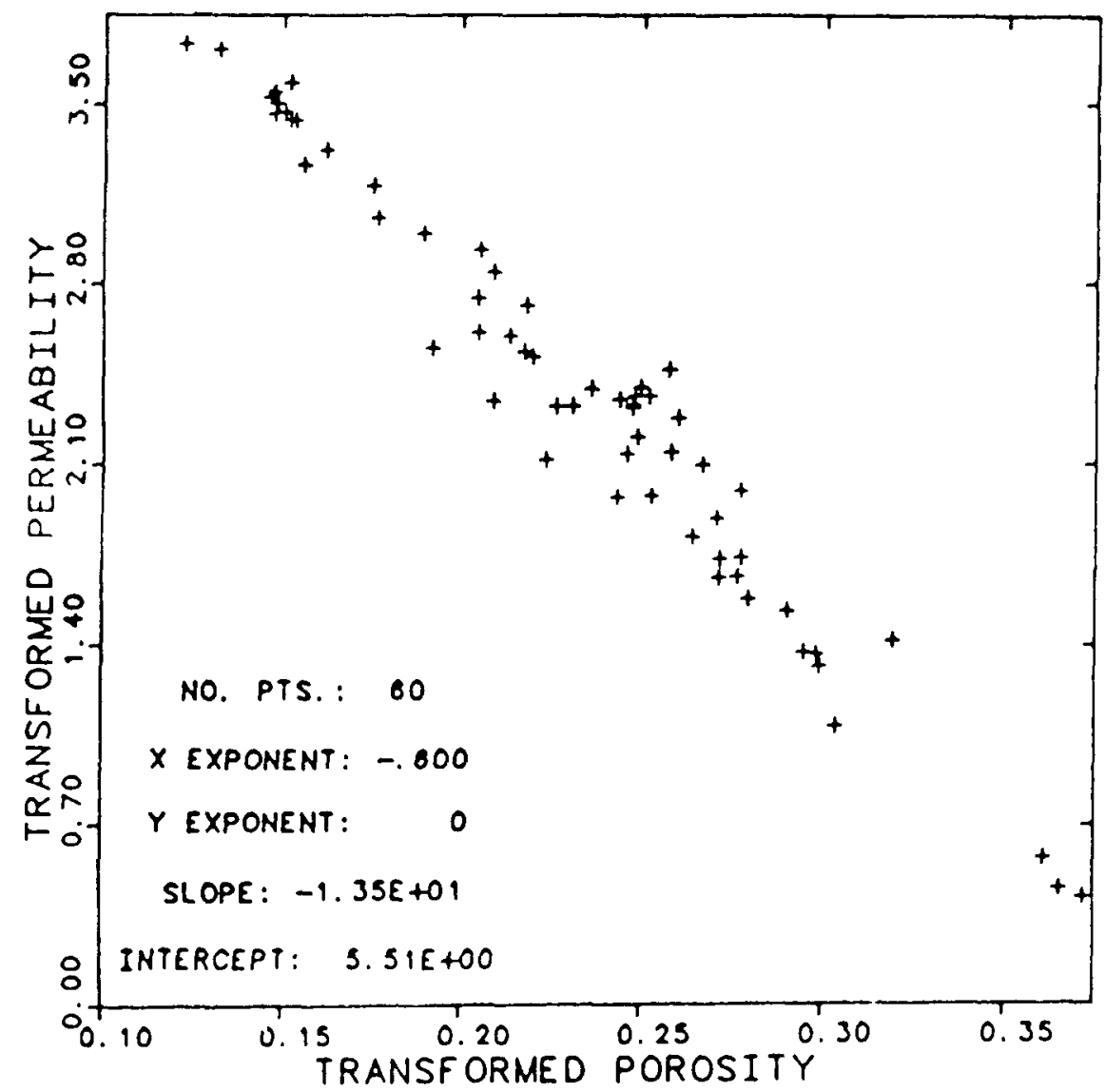

Figure III-8. Porosity-permeability relation for transformed Fontainebleau data.

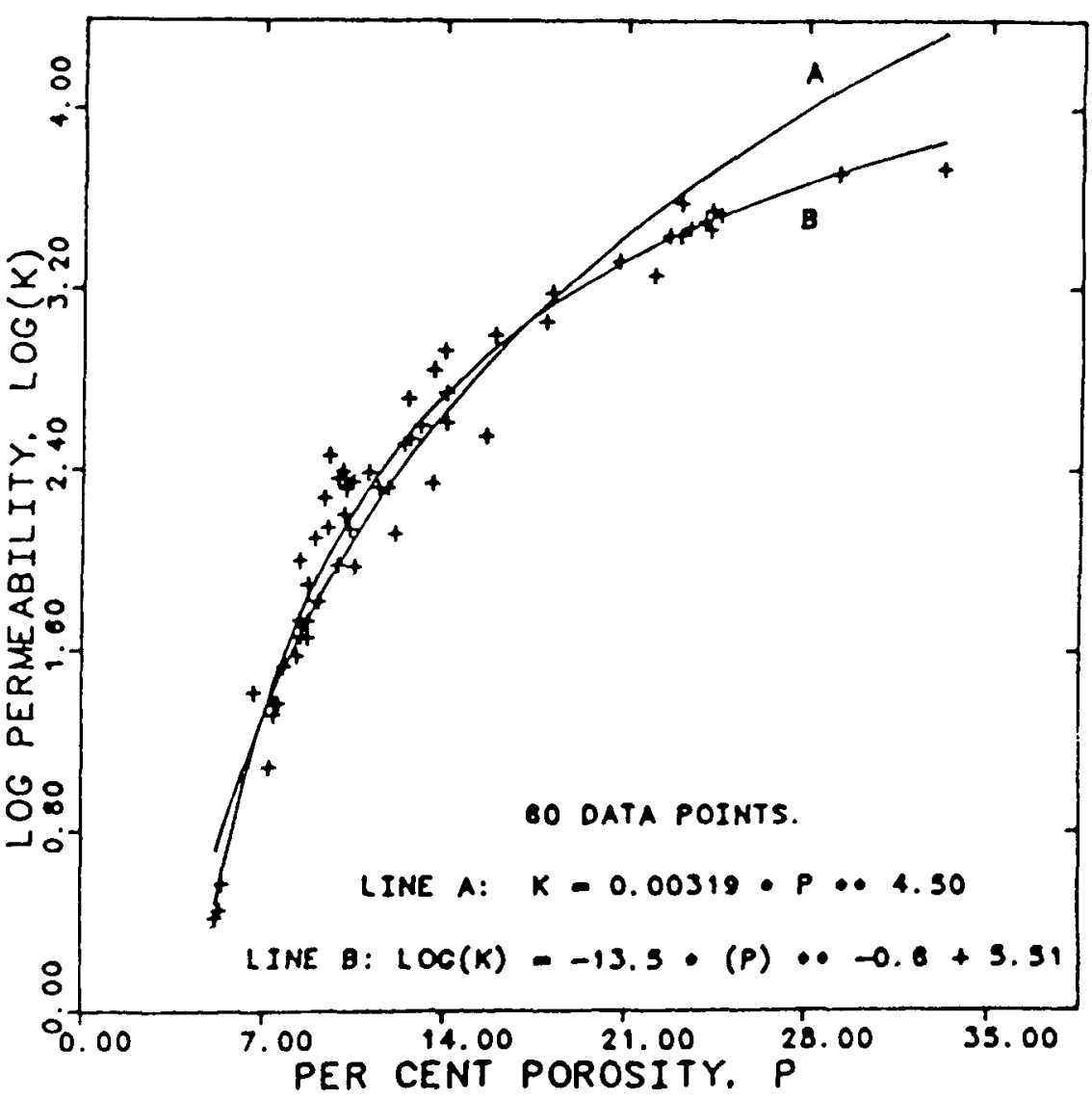

Figure III-9. Fontalnebleau data and predictive curves. 


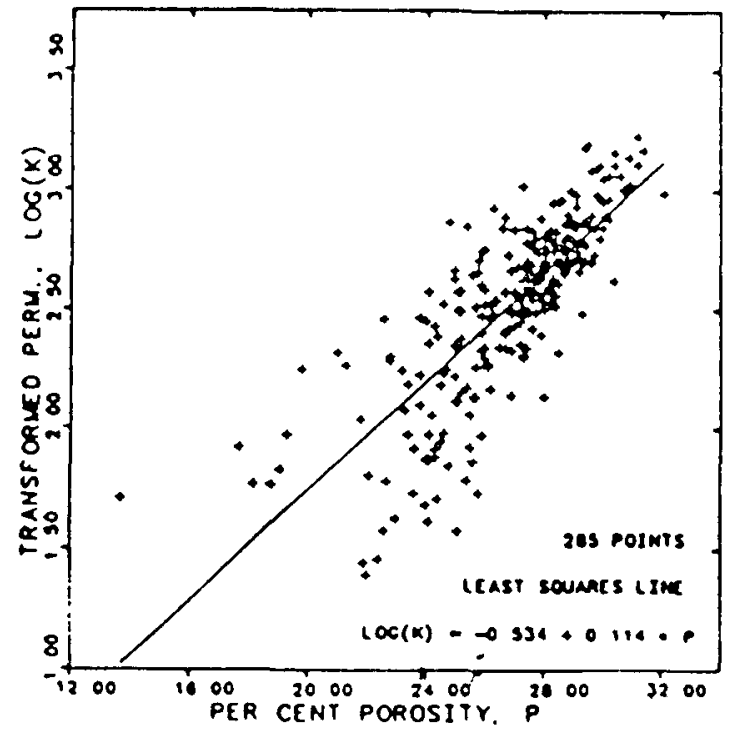

Figure III-11. Chesney lease porosity and permeability data.

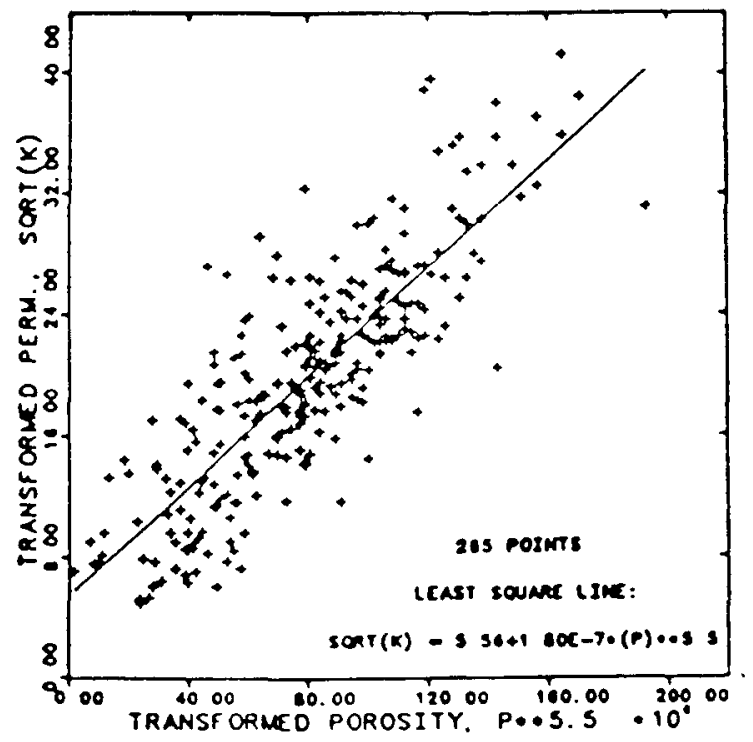

Figure III-10. Chesney lease porosity and permeability data.

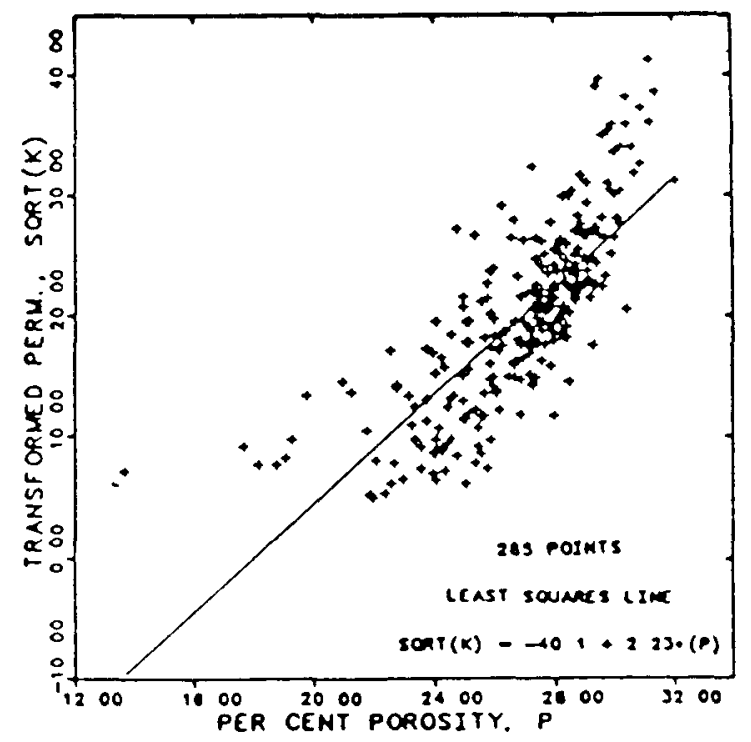

Figure III-12. Chesney lease porosity and permeability data. 
is much greater at low porosities than at high porosities. The points at the extremes of the porosity range have the largest influence on the least squares line (Hoaglin et al., 1983). One might be tempted to use weighted least squares for the regression procedure because of the large change in variability. Note that the datum at $\phi=13.7$ might be branded as an outlier or inconsistent datum from this plot. The $\phi$ versus $\log (k)$ plot has a coefficient of correlation of 0.791 .

Figure III-11 shows some improvement in terms of homogeneity; the variation in permeability at fixed porosity is more even than for Fig. III-10. The cloud of data has a concave upward appearance, suggesting that a linear regression model may not be adequate. The coefficient of correlation is 0.774 , indicating a diminishment of the linear nature of the relationship from Fig. III-10.

Figure III-12 shows a fairly constant variability in permeability with porosity. A weighted least squares regression is not necessary for this case. The "out1ier" (or "rogue" as they are sometimes called) at $\phi=13.7$ no longer looks out of place. The coefficient of correlation is estimated to be $\rho=0.826$.

From Figs. III-10 and III-12 and their associated coefficients of correlation, a wide variation in behavior is reflected in very small changes in $\rho$. The value of $\rho$ has a statistical interpretation only when $(\phi)^{\mathrm{q}}$ and $(\mathrm{k})^{\mathrm{p}}$ are b.n.d. A high value of $|\rho|$ indicates that the $\phi-k$ relationship has a linear component. Whether the simple least squares regression captures this Iinearity depends on more than $|\rho|$ being large; the relative positions of the points have an influence on the regression lines. 
Using the approach previously outlined, we used the regression curve in Fig. III-12 to predict permeability based on porosity. The results are shown in Fig. III-13 as curve A. The correction for bias using Eq. (13), $\sigma_{y}^{2}\left(1-\rho^{2}\right)=(7.32)^{2}\left[1-(0.826)^{2}\right]=+17 \mathrm{md}$, is small for this data set. Curve $B$ is computed from the line of regression in Fig. III-10; it underestimates (relative to curve A) the permeability by 5 to 80 percent. As in case 1, the improvement in fit is primarily in the low and high permeability regions of the plot. If these regions of the reservoir are not important, then either curve $A$ or $B$ suffices to predict permeability. On the other hand, if the low and high permeability regions are of particular interest (e.g. profile control or establishing a net pay cutoff), then the error in prediction would be reduced and the extra computational effort is justified.

To determine the impact of selecting curve $A$ over curve $B$ to predict permeability, Monte Carlo simulations were performed to compare the average reservoir permeability predicted using each curve. The simulations were performed in the following manner.

1) Randomly select $N$ core plugs from the data set (with replacement), each having a porosity $\phi_{1}$ and a permeability $k_{1}$, $i$ $=1,2, \ldots, N$.

2) Predict the core plug permeabilities using curves $A$ and $B$ :

$$
\begin{aligned}
& \hat{\mathrm{k}}_{\mathrm{iA}}=\left(5.56+1.80 \times 10^{-7} \phi_{i}^{5.5}\right)^{2}+17.0, \\
& \hat{\mathrm{k}}_{\mathrm{iB}}=(10)^{-0.534+0.114 \phi_{i} .}
\end{aligned}
$$




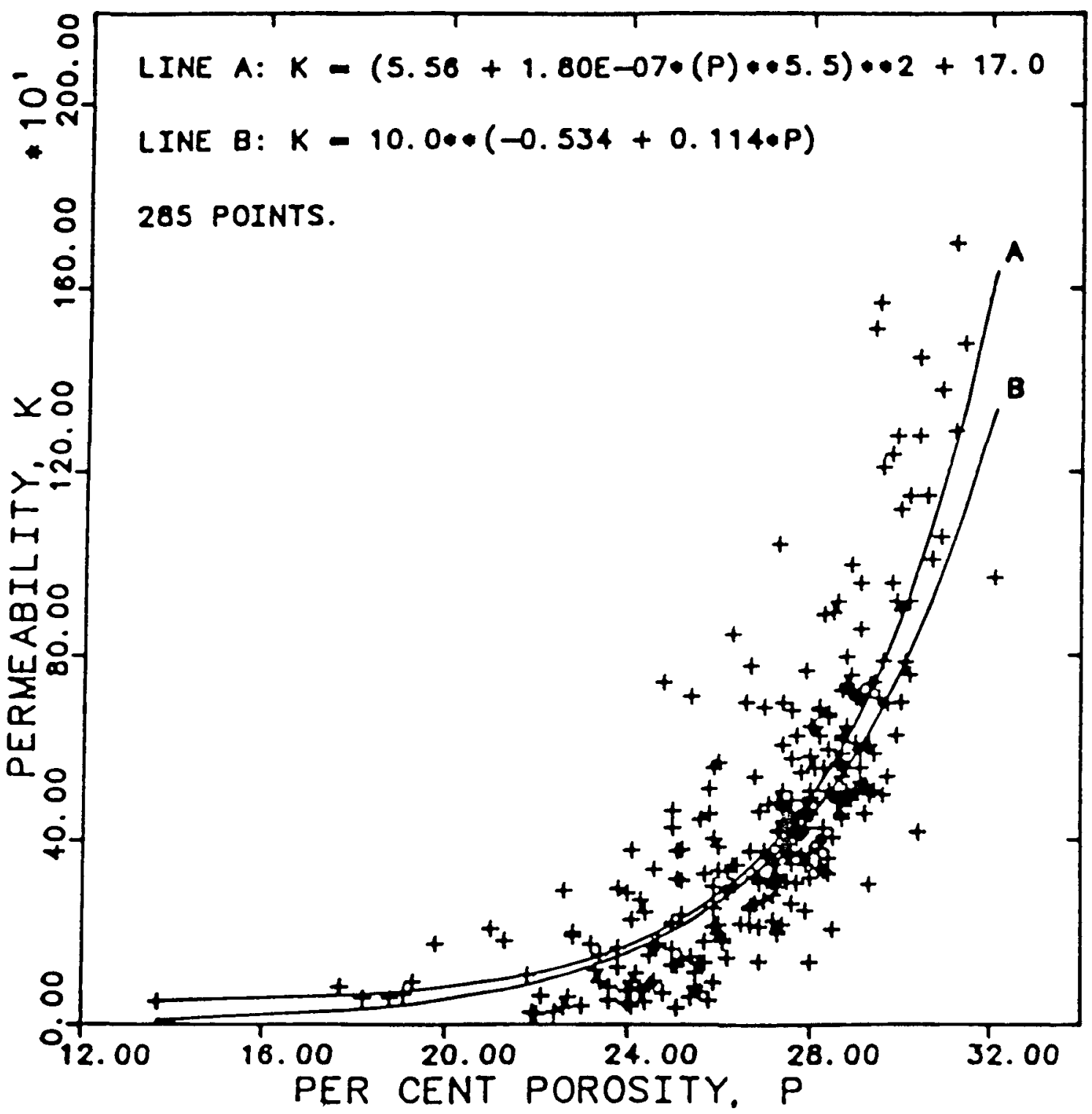

Figure III-13. Chesney data and predictive curves. 
3) Arithmetically average the various estimates:

$$
\begin{aligned}
& \overline{\mathrm{k}}=\frac{1}{\mathrm{n}} \Sigma \mathrm{k}_{\mathrm{i}}, \\
& \overline{\mathrm{k}}_{\mathrm{A}}=\frac{1}{\mathrm{n}} \Sigma \hat{\mathrm{k}}_{i \mathrm{~A}}, \\
& \overline{\mathrm{k}}_{B}=\frac{1}{\mathrm{n}} \Sigma \hat{\mathrm{k}}_{\mathrm{iB}},
\end{aligned}
$$

where the summations are over $\mathrm{n}$ terms.

4) Compute the relative error in the estimates:

$$
\begin{aligned}
& e_{A 1}=\left(\bar{k}_{A}-\bar{k}\right) / \bar{k} \\
& e_{B 1}=\left(\bar{k}_{B}-\bar{k}\right) / \bar{k} .
\end{aligned}
$$

5) Perform steps (1) - (4) another m-1 times and compute the average relative error,

$$
\begin{aligned}
& \overline{\mathrm{e}}_{\mathrm{A}}=\frac{1}{\mathrm{~m}} \Sigma \mathrm{e}_{\mathrm{Ai}} \\
& \overline{\mathrm{e}}_{\mathrm{B}}=\frac{1}{\mathrm{~m}} \sum \mathrm{e}_{\mathrm{Bi}}
\end{aligned}
$$

and the variability of the error,

$$
\begin{aligned}
& s_{A}^{2}=\frac{1}{m-1} \Sigma\left(e_{A i}-\bar{e}_{A}\right)^{2} \\
& s_{B}^{2}=\frac{1}{m-1} \Sigma\left(e_{B i}-\bar{e}_{B}\right)^{2}
\end{aligned}
$$

where the summations are over $m$ values.

Table III- 1 shows the Monte Carlo results for $m=1000$ and all 285 core plug values. The error diminishes for increasing numbers of 
TABLE III-1. Monte Carlo simulation results for 285 core plug values.

$\begin{array}{rll}\frac{N}{3} & & \frac{\bar{e}_{A}}{n} \\ 5 & & 0.060 \\ 10 & & 0.033 \\ 20 & & 0.017 \\ & & 0.0083\end{array}$
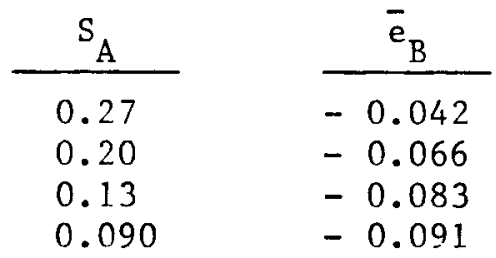

$\frac{S_{B}}{0.25}$
0.18
0.12
0.082

TABLE III-2. Monte Carlo simulation results for core with $\phi \geqq 28.85$

$\begin{array}{rll}\frac{N}{3} & & \frac{\bar{e}_{A}}{n} \\ 5 & & 0.013 \\ 10 & & 0.00035\end{array}$

\begin{tabular}{l}
$\mathrm{S}_{\mathrm{A}}$ \\
\hline 0.19 \\
0.15 \\
0.10
\end{tabular}

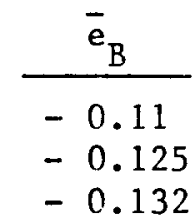

$\frac{S_{B}}{0.17}$
0.13
0.090

TABLF III-3. Monte Carlo simulation results for 285 core plug values

\begin{tabular}{|c|c|}
\hline $\mathrm{N}$ & $\mathbf{e}_{\mathrm{A}}$ \\
\hline 3 & 0.17 \\
\hline 5 & 0.15 \\
\hline 10 & 0.14 \\
\hline 20 & 0.13 \\
\hline
\end{tabular}

\begin{tabular}{c}
$\mathrm{S}_{\mathrm{A}}$ \\
\hline 0.36 \\
0.26 \\
0.18 \\
0.13
\end{tabular}

\begin{tabular}{c}
$\overline{\mathbf{e}}_{\mathrm{B}}$ \\
\hline 0.048 \\
0.030 \\
0.022 \\
0.010
\end{tabular}

\begin{tabular}{c}
$S_{B}$ \\
\hline 0.33 \\
0.24 \\
0.17 \\
0.12
\end{tabular}


predictions (N) when curve $A$ is used. Curve $B$ underestimates the permeability by an increasing proportion as $n$ increases. Since both curves approximate well the porosity-permeability relationship in the central portion $(24 \leqq \phi \leqq 29)$ of the data, the better predictive power of curve A shows when there is a good chance that a high (or low) porosity core plug is chosen (i.e $N$ large). Comparing $S_{A}$ and $S_{B}$ values, both curves have about the same efficiency in prediction.

Since delineation of high permeability zones is important for some purposes, another Monte Carlo simulation was made to compare the performance of curves $A$ and $B$ for the top twenty percent of the data. Only core plugs with porosities in excess of 28.85 were included, giving 58 values for use in the simulations. Table III-2 shows the results for $m=200$. The average error is diminished by at least one order of magnitude using curve A.

The performance exhibited in Tables III-1 and III-2 for each curve reflects, in part, the properties of the data set. The improvement for other fields may be greater or less than demonstrated for this data set. However, until a plot such as Fig. III-13 is made, it is not clear how great the benefit may be. The results also depend on combining the predictions in a linear manner, such as the arithmetic average.

As previously discussed, nonlinear combinations of predictions like the geometric average may influence substantially the error behavior. To demonstrate this, Monte Carlo simulations were performed using the following formulae for step (3): 


$$
\begin{aligned}
& \overline{\mathrm{k}}=\left(\Pi \mathrm{k}_{\mathrm{i}}\right)^{1 / \mathrm{N},}, \\
& \overline{\mathrm{k}}_{\mathrm{A}}=\left(\pi \hat{\mathrm{k}}_{\mathrm{iA}}\right)^{1 / N},
\end{aligned}
$$

and

$$
\bar{k}_{B}=\left(\pi \hat{k}_{i B}\right)^{1 / N} \text {, }
$$

where the products are over $n$ terms. That is, the geometric averages of the permeability estimates were used in the computations. The results for $\mathrm{m}=1000$ and 285 core plug values are shown in Table III-3. The performance is quite different from that shown in Table III-1. The estinates from curve A are biased considerably.

Case 3 -This data set also comes from a deltaic sand. A geologic study by Boyer (1984) indicates that well 400 -- the source of these core plug data -- is located at the eastern edge of the Sims Sandstone Unit in the proximal region. Figure III-4 is a plot of the porosity and permeability data.

An analysis of the porosity and permeability distributions shows the porosity to be approximately normal while the permeability is approximately log-normal. Consequently, a simple linear regression of the form $\ln (k)=a \cdot \phi+b$ appears to be appropriate. The method of least squares gives $\mathrm{a}=0.271$ and $\mathrm{b}=-0.864$ and this line is shown in Fig. III-14. The coefficient of correlation, $\rho$, is estimated to be 0.706 and the variance $\sigma_{x}^{2}$ is about 0.822 for this data set. 


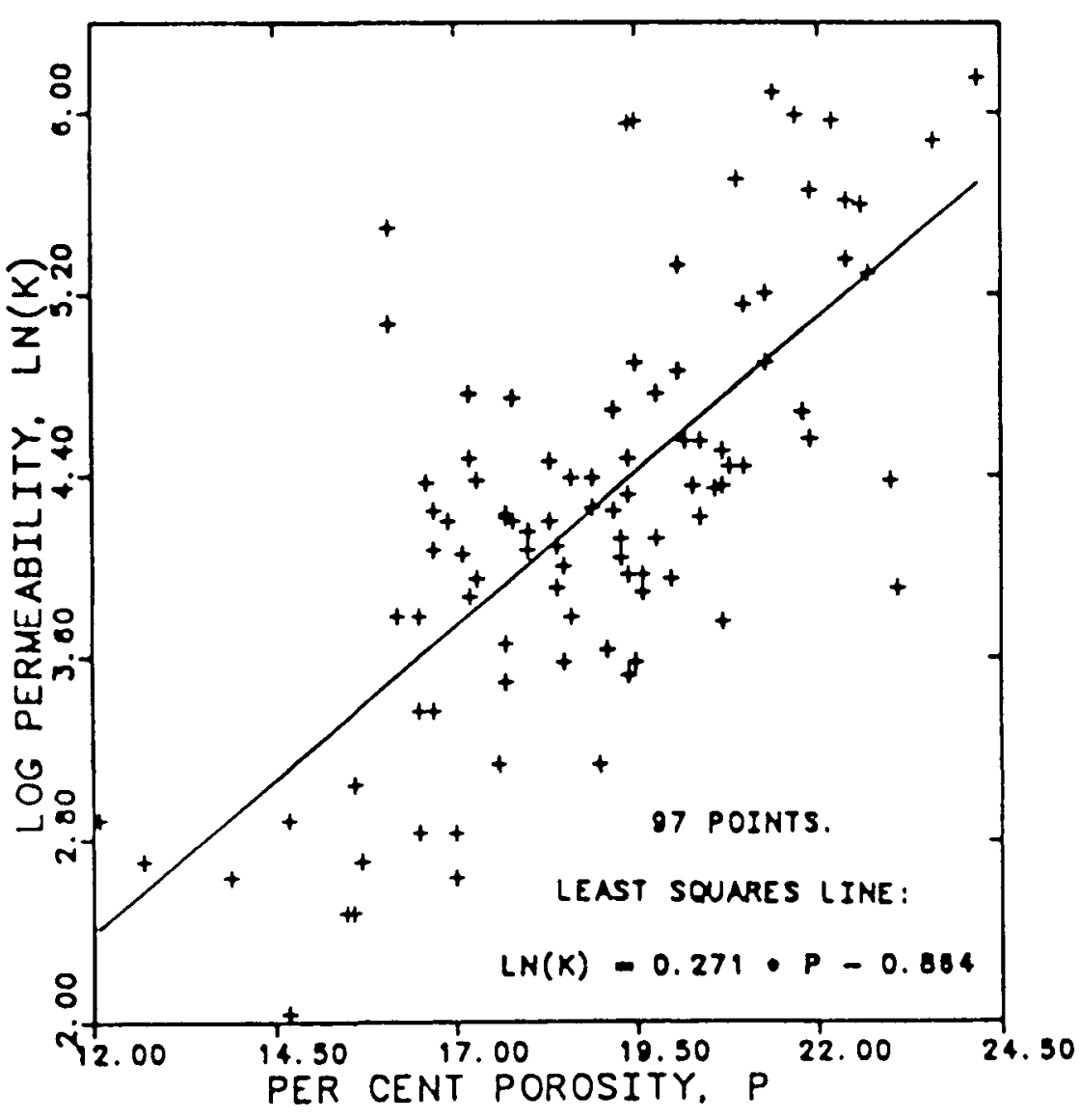

Figure III-14. Porosity and permeability data for well 400 , Sims Sand

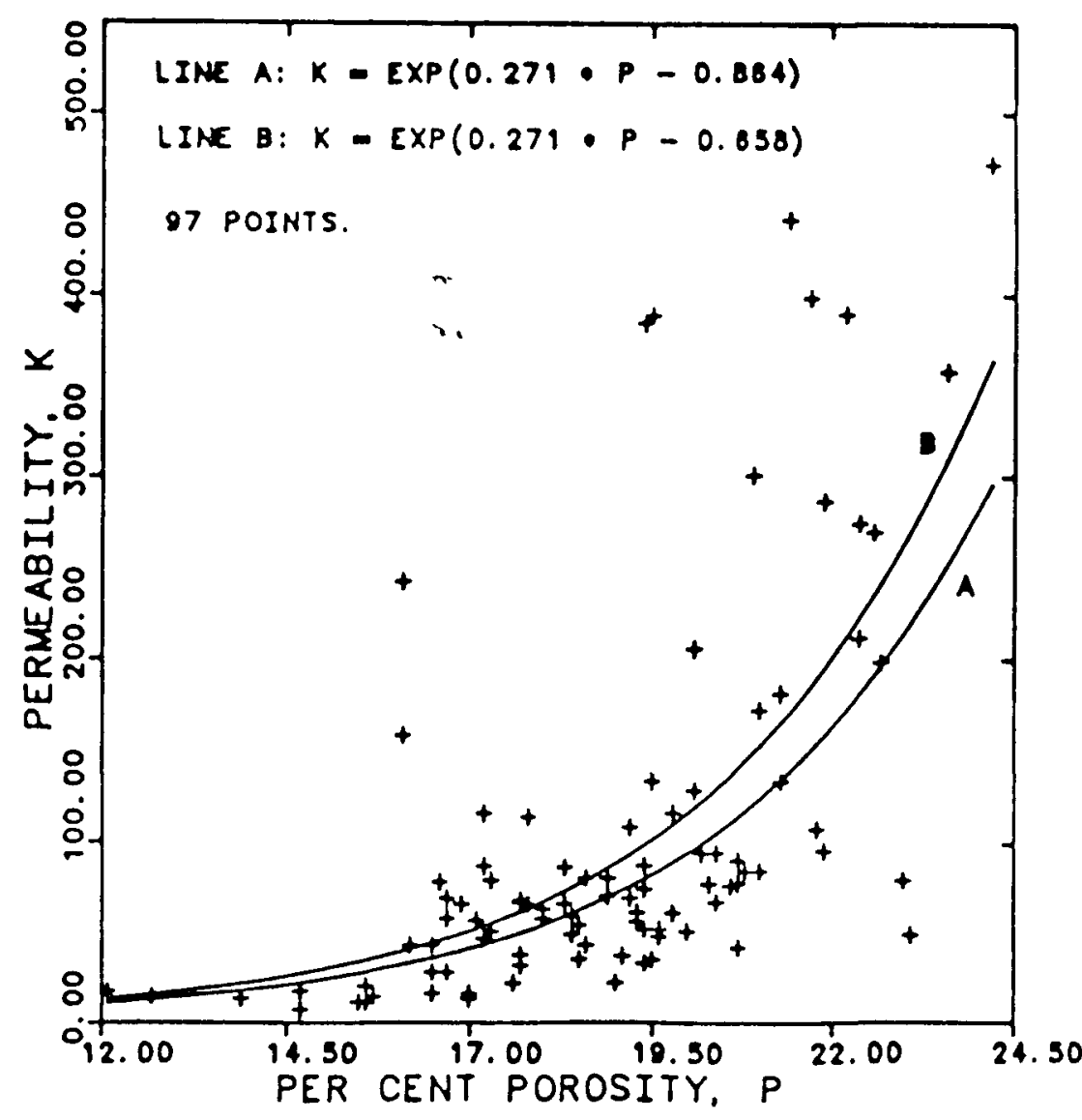

Figure III-15. Sims Sand data and predictive curves. 
We have all the data we need for Eq. (III-10) to be used in the prediction of permeability from porosity. We begin with $\sigma^{2}=\sigma_{x}^{2}(1-$ $\left.\rho^{2}\right)$

or $\quad \sigma^{2}=(0.822)\left[1-(0.706)^{2}\right]$

to give $\sigma^{2}=0.412$. This result in Eq. (III-10) gives

$$
\hat{\mathrm{k}}=\exp \{0.271 \cdot \phi-0.864+0.5 \cdot 0.412\}
$$

or $\hat{\mathrm{k}}=1.23 \exp \{0.271 \cdot \phi-0.864\}$.

Thus, to correct for the nonlinear exponential transformation, the permeability predicted by the regression line is increased by 23 percent. Figure III-15 shows the two lines on a porosity-permeability plot. Line $A$ has no correction for bias while line $B$ includes the correction.

Concluding Remarks - This section has reviewed some of the salient features of prediction from regression-based porosity-permeability models. The issues of which model to choose, estimator bias, and use of the estimates have been shown to be influential in the optimal prediction of permeability.

Power transformations of data are a useful method for obtaining optimal permeability estimates. Transforming data may give new random variables having desirable properties such as simplifying the model, allowing standard statistical tests to be used, and correcting for 
transformation of the data. Data transformations have also been seen to equalize the variability of the data across the range of porosity values, thereby reducing the need for a weighted least squares regression. Discordant data points (outliers) may not, in fact, appear discordant when the appropriate data transformation is applied. 
THE INFLUENCE OF SAMPLE SIZE AND PERMEABILITY DISTRIBUTION

UPCN HETEROGENEITY MEASURES

Introduction - Reservoir heterogeneity has long been recognized as being an influential factor in determining reservoir performance. Variations in any reservoir property can degrade the efficiency of a recovery process. The variability of permeability, however, appears to be particularly influential. We restrict our attention this section to permeability heterogeneity.

The two traditional methods of quantifying heterogeneity are the Dykstra-Parsons (1950) coefficient, $\mathrm{V}_{\mathrm{DP}}$, and the Lorenz (Schmalz and Rahme, 1950) coefficient, $\mathrm{L}_{\mathrm{C}}$. Both measures have values between zero and one, with higher heterogeneity being represented by the larger numbers and zero signifying a homogeneous reservoir. Both scales are such that cases of low heterogeneity occupy the range zero to about one-half, while the moderate and high heterogeneity cases occupy the remining half of the scales. Modelling studies (Dykstra and Parsons, 1950; Schmalz and Rahme, 1950; Warren and Cosgrove, 1964; Koval, 1963; Craig, 1971; Khan, 1971; Arya et a1., 1985) using these measures show that performance is insensitive to the precise value of $\mathrm{V}_{\mathrm{DP}}$ or $\mathrm{L}_{\mathrm{C}}$ for low heterogeneity cases. High heterogeneity however, exhibit a strong relationship between performance and $\mathrm{V}_{\mathrm{DP}}$ or $\mathrm{L}_{\mathrm{C}} \cdot$ For example, the Dykstra and Parsons waterflood model shows that, for a producing water-oil ratio of 100 and a unit mobility ratio, an increase of $V_{D P}$ from 0.2 to 0.3 decreases the fractional oil recovery from 0.57 to 0.54 , a change of five percent. A similar increase of $V_{D P}$ from 0.8 to 0.9 , however, decreases the oil recovery from 0.35 to 0.25 , a change 
of 33 percent. This feature leads to a problem with these coefficients: the performance of a model may become very sensitive to the precise value used for the coefficient.

A question then arises in the use of $\mathrm{V}_{\mathrm{DP}}$ or $\mathrm{L}_{\mathrm{C}}$ for moderate to high heterogeneity cases. A coefficient is evaluated on the basis of a limited number of permeability samples from the reservoir popuiation. Since permeability is a random variable, the calculated coefficient is only an estinate of the true reservoir coefficient. Usually this estimate will not have the same value as the true coefficient. For a small number of data, the error may be quite large. Intuitively, we would expect that, as the number of data increases, the computed value approaches the true value. But, if these coefficients are to be useful as indicators of performance, we should know how many data are needed to obtain a "sufficiently accurate" estimate.

Another issue is that both $\mathrm{V}_{\mathrm{DP}}$ and $\mathrm{L}_{\mathrm{C}}$ are one-parameter measures of permeability variability. Either coefficient can be estimated from permeability data indeperdently of how the data are distributed. (Although the Dykstra-Parsons procedure uses a log-normal probability plot, a best-fit line is used to determine the coefficient.) Several studies (Lambert, 1981; Goggin et al., 1986; Jensen et al. 1985), however, have shown that permeability exhibits various distributions. How good are $\mathrm{V}_{\mathrm{DP}}$ and $\mathrm{L}_{\mathrm{C}}$ at predicting the behavior of displacements in reservoirs having different permeability distributions?

The general issues of estimate reliability versus sample size and estimate sensitivity to the underlying probability density function (p.d.f.) have been recognized for many years in the statistics 
literature. However, very little appears to have been done to assess the Nykstra-Parsons and the Lorenz coefficients in these respects. Lambert (1981) studied the effects of sample size on coefficient estimates by computing both coefficients for each of several wells. She found that, as the number of samples decreased, the variability of the estimate consistently increased.

This study presents analyses of $\mathrm{V}_{D P}$ and $\mathrm{L}_{C}$ for their behavior as functions of sample size and permeability distribution. On average, the estimates underestimate the true population coefficient. The variability of the estimates increases as the sample size decreases. The coefficients may also be insensitive to changes in the probability density function. Some example cases are considered to illustrate the impact of these insensitivities to predicting reservoir performance. In view of these findings, we propose a new heterogeneity measure which is less sensitive to the numbers of samples and which accounts for the permeability p.d.f. The relationships between this new measure and the Dykstra-Parsons and Lorenz coefficients are given.

Sample Size Effects on Heterogeneity Measures - We begin by defining the terms bias, standard error, and efficiency. The $\mathrm{V}_{\mathrm{DP}}$ and $\mathrm{L}_{\mathrm{C}}$ estimators are studied to determine how the bias and standard error of each varies with the sample size. Several cases are then cited to show the impact estimate error may have on reservoir performance.

The two criteria used here to judge the performance of estimators are bias and standard error (Kenda11 and Stuart, 1977). Consider the case where we have a large number ( $r$ ) of data sets, all coming from the same population, and which have the same number of data ( $n$ ) in 
each set. We estimate the population parameter ( $z$ ) by computing $r$ and estimates $\hat{z}_{i}, i=1,2, \ldots, r$. If, over all $r$ data sets, the estimates $\hat{z}_{i}$ are found to systematically over or underestimate the true vaiue of $z$, the estimator is said to be biased. An unbiased estimator is desirable, but is not necessarily the most important feature since we may be able to apply a correction factor for the bias.

The estimates $\hat{z}_{i}, i=1,2, \ldots, r$, will differ from $z$ by varying amounts. Usual1y, one assumes that $\Delta_{z_{i}}=\hat{z}_{i}-z$ is $N\left(m_{z}, s_{z}^{2}\right)$. That is, the errors $\Delta z_{i}$ are normally distributed with mean $m_{z}$ and variance $\mathrm{s}_{z}^{2}$. When $\mathrm{m}_{\mathrm{z}}=0$, the estimator is unbiased. $s_{z}$ is the standard error of the estimator. The normal p.d.f. implies that estimator $\left|\hat{z}_{i}-m_{z}\right|$ $<s_{z}$ for about 67 percent of the samples and that $\left|\hat{z}_{i}-m_{z}\right|<2 s_{z}$ for 95 percent of the samples (Kendall and Stuart, 1977). $s_{z}$ is usually a function of $n$, with $s_{z}$ decreasing as $n$ increases. Given two methods to estimate $z$ from a data set, the method having the lower standard error is more efficient.

Sample Size Effects on the Dykstra-Parsons Coefficient - In Appendix $A$, expressions are developed for the bias, $m_{v}$, and standard error, $s_{v}$, of the Dykstra-Parsons coefficient estimate $\hat{V}_{\mathrm{DP}}$. For the case where the permeability p.d.f. is log-normal, they are

$$
\mathrm{m}_{\mathrm{v}}=-0.749\left[\ln \left(1-\mathrm{v}_{\mathrm{DP}}\right)\right]^{2}\left(1-\mathrm{v}_{\mathrm{DP}}\right) / \mathrm{N}
$$

and

$$
s_{v}=-1.49\left[\ln \left(1-V_{D P}\right)\right]\left(1-V_{D P}\right) / \sqrt{N}
$$


where $N$ is the number of data in the sample and $V_{D P}$ is the Dykstra-Parsons coefficient of the permeability distribution. These equations assume that $\mathrm{N}$ is large, but they give good approximations when $N \geqq 20$ (Jensen, 1986).

Equation (III-11) indicates that the estimate $\hat{V}_{D P}$ is biased; the estimator underestimates the true coefficient $\left(m_{v}<0\right)$. The bias $m_{v}$ is proportional to $\mathrm{N}^{-1}$ and reaches a maximum (in absolute value) when $\mathrm{V}_{\mathrm{DP}}=0.87$. The bias is small. For example, $\mathrm{m}_{\mathrm{v}}=0.02$ for $\mathrm{N}=20$ and $V_{D P}=0.87$. Hence, for any reasonably sized log-normal data set, the bias $\mathrm{m}_{\mathrm{v}}$ is insignificant.

Equation (III-12) on the other hand, shows that $\mathrm{s}_{\mathrm{v}}$ is proportional to $\mathrm{N}^{-1 / 2}$ and attains a maximum for $\mathrm{V}_{\mathrm{DP}}=0.63$. Figure III-16 shows the number of samples $n$ required to obtain a given standard error as $V_{D P}$ varies between 0.3 and 0.9 . For example, to maintain $s_{v}=0.05$, a data set of 50 or more samples is always required and 120 samples are needed when $\mathrm{V}_{\mathrm{DP}}=0.6$.

The curves in Fig. III-16 are concave downward because of two competing effects on the $V_{D P}$ estimator. As formation heterogeneity increases, more samples are needed to adequately define the variability. This effect is shown in the positive-slope $\left(V_{D P}<0.6\right)$ portion of the curves. For $\mathrm{V}_{\mathrm{DP}}>0.6$, the increasing insensitivity of $\mathrm{V}_{\mathrm{DP}}$ to changes in heterogeneity level dominates. This is the same behavior described in the introduction; when $V_{D P}$ is large, the heterogeneity must change substantially for $V_{D P}$ to be perturbed. Hence, fewer samples are required to define the variability for high heterogeneity cases. 


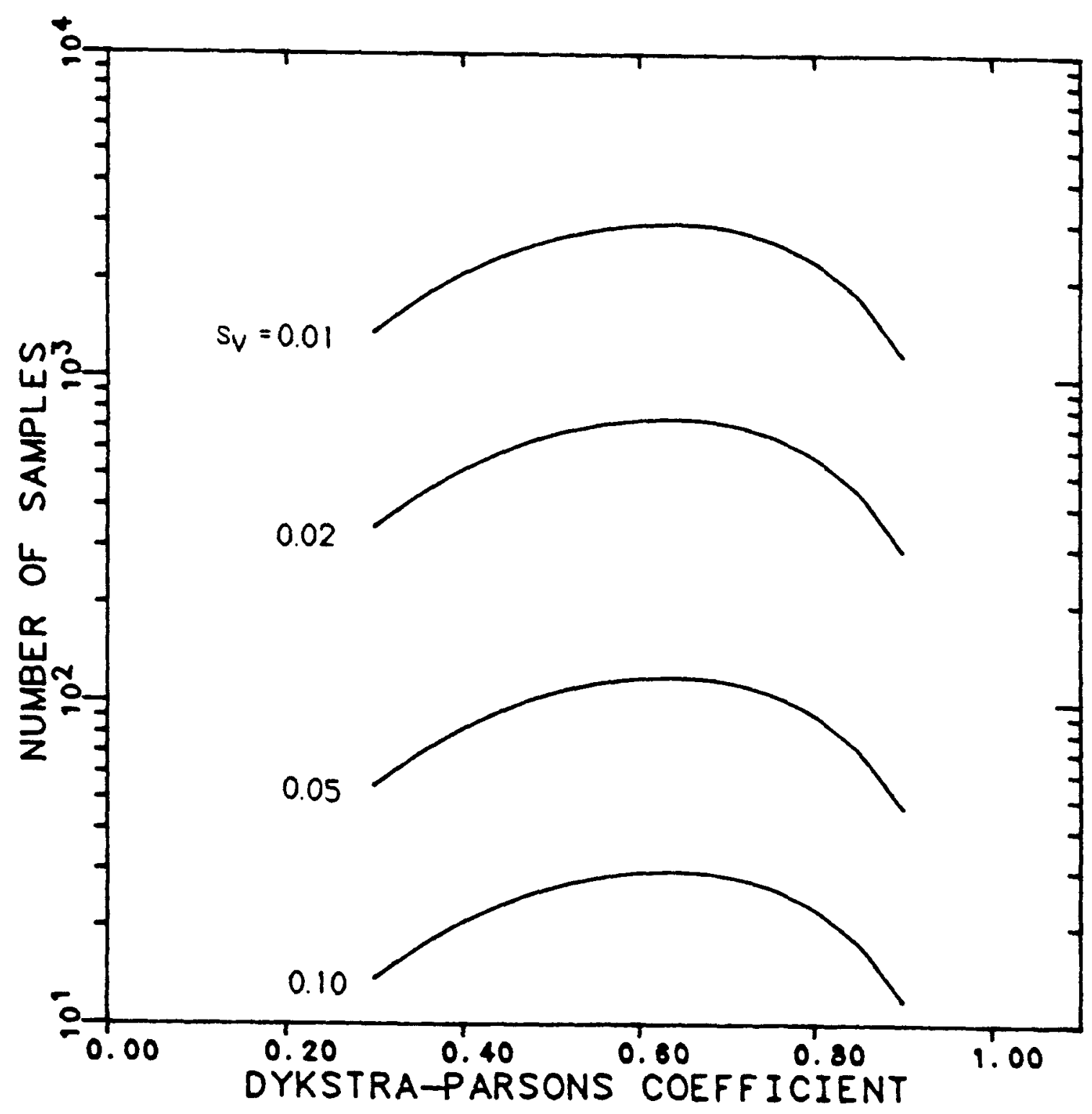

FIgure III-16. Sample size behavior for the Dykstra-Parsons estimator. 
To use Fig. III-16 for a real situation, $\hat{\mathrm{V}}_{\mathrm{DP}}$ would have to be used instead of $\mathrm{V}_{\mathrm{DP}}$. Knowing the number of data at hand, an estimate of $s_{v}$ may be made. This $s_{v}$ estinate indicates the error associated with $\hat{V}_{D P}$ and allows a decision regarding the data set sufficiency to be made.

Sample Size Effects on the Lorenz Coefficient - An analytical solution to the sampling error of the $L_{C}$ estimator is a much more difficult task than for $V_{D P}$ because it involves integration of the area between two curves. In the statisitcal and economics literature, the Lorenz coefficient appears to be evaluated using Gini's coefficient of concentration (Kenda11 and Sturat, 1977) rather than evaluate the area between two lines (Gail and Gastwirth, 1978; Gastwirth, 1972). Since the practice of the petroleum industry is to estimate $\mathrm{L}_{\mathrm{C}}$ using integration and no theory is avallable, we have used Monte Carlo simulation to address the problem.

Appendix $B$ describes the procedure and associated equations used to simulate the Lorenz sampling problem. We assume a log-normal permeability p.d.f. describes the population to provide results for a typical situation. Figure III-17 shows the bias results for the trapezoidal method of numerical integration. The vertical axis is scaled as $\mathrm{m}_{\mathrm{L}} / \mathrm{L}_{\mathrm{C}}$ to show the bias as a fraction of the true Lorenz coefficient. Just as $\mathrm{m}_{\mathrm{v}}$ is a function of $\mathrm{V}_{\mathrm{DP}}$, the bias $\mathrm{m}_{\mathrm{L}}$ of the Lorenz estimator depends on the true value of $\mathrm{L}_{\mathrm{C}}$ for the population. The bias is always negative and is signiffcant $\left(\mathrm{m}_{L} / L_{C}<-0.06\right)$ for small data sets $(N<40)$ and heterogeneous distributions $\left(L_{C}>0.6\right)$. 


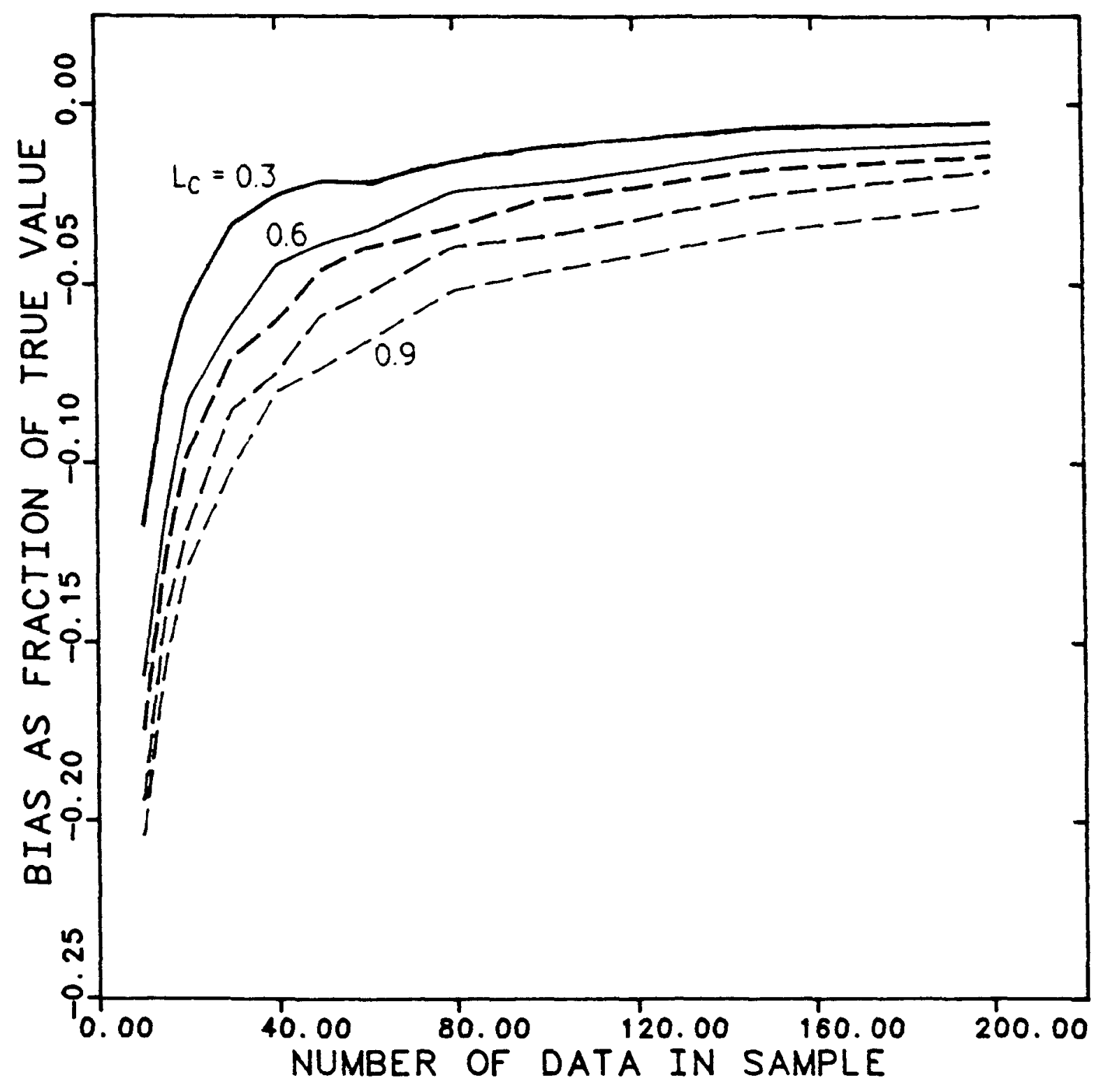

Figure III-17. Bias performance for the Lorenz estimator. 
A similar computation using Simpson's rule showed that the bias is insensitive to the method of integration.

Figure III-18 shows the number of points required to maintain the standard error $s_{L}$ of the estimator at given values for $0.3 \leqq L_{C} \leqq 0.9$. For large $s_{L}$, the estimator is quite efficient (requires few points); for small $s_{L}$, the estimator requires a large amount of data. The problem of significant bias of the Lorenz estimator makes the use of Fig. III-18 slightly complicated. Because $\hat{\mathrm{L}}_{\mathrm{C}}$ (and not $\mathrm{L}_{\mathrm{C}}$ ) is available, if $\hat{\mathrm{L}}_{\mathrm{C}}$ is used for the abcissa of Figure TII-18, the number of points and the standard error may be underestimated. A "compensated" value, e.g. $1.05 \cdot \hat{\mathrm{L}}_{\mathrm{C}}$, would probably be more appropriate as an estimate for $\mathrm{L}_{\mathrm{C}}$.

Comparison of the Dykstra-Parsons and Lorenz Estimators - In terms of the bias, both the $\mathrm{V}_{D P}$ and $\mathrm{L}_{C}$ estimators underestimate the true value of the underlying population. The Lorenz estimator, however, gives and estimate that is considerably more biased than the Dykstra-Parsons estimator. This problem is aggravated because there is no analytic expression for the Lorenz estimator bias. Otherwise, a correction factor could be introduced.

For an assessment of which estimator is more efficient, caution should be used when comparing Figs. III-16 and III-18. This is because $\mathrm{L}_{\mathrm{C}}$ and $\mathrm{V}_{\mathrm{DP}}$ have different scales, although the endpoint values $(0$ and 1) are the same. When the permeability is log-normally distributed,

$$
\mathrm{L}_{\mathrm{C}}=\operatorname{erf}\left[-0.5 \cdot \ln \left(1-\mathrm{V}_{\mathrm{DP}}\right)\right]
$$




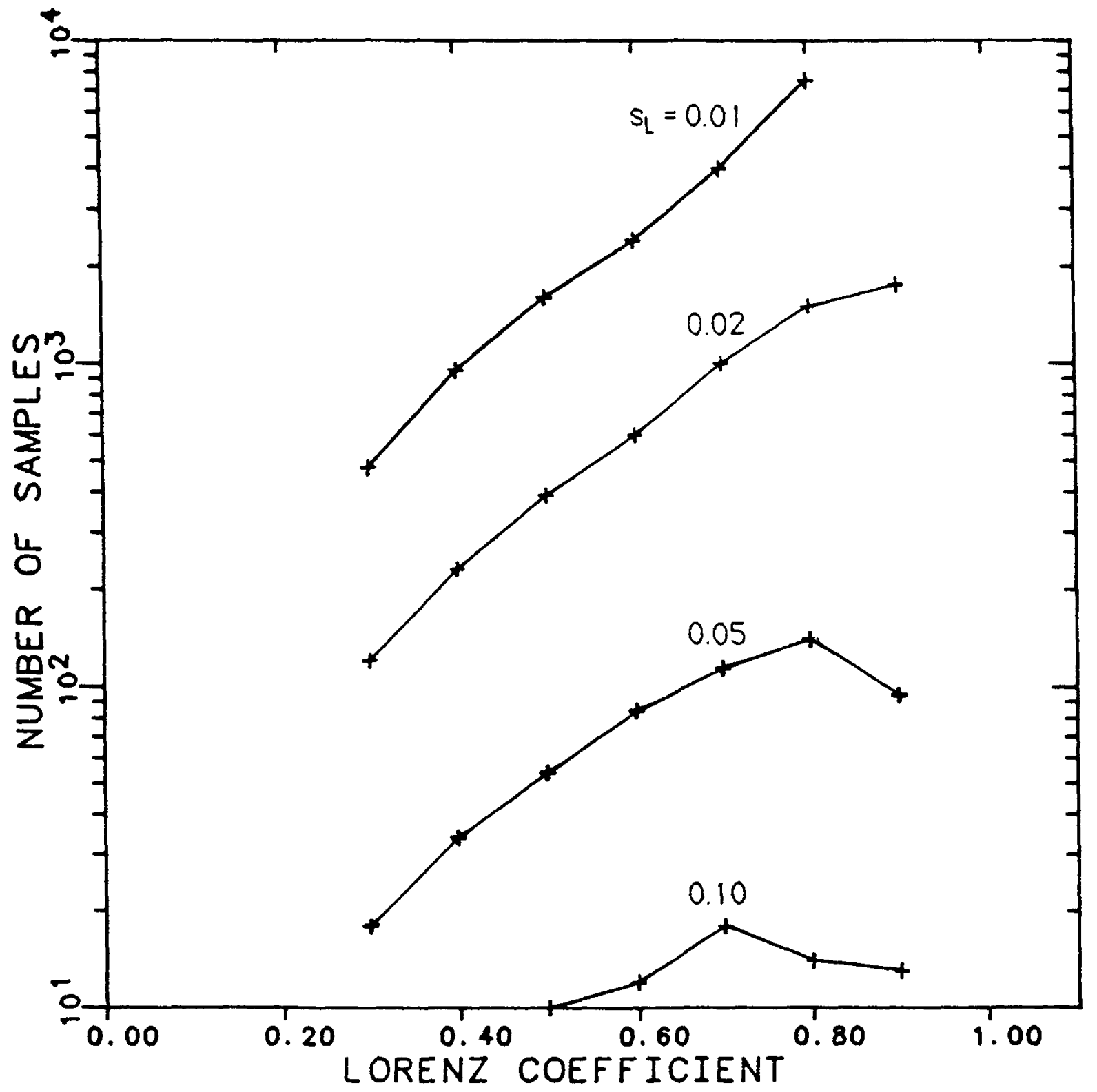

Figure III-18. Sample size behavior for the Lorenz estimator. 
where erf is the error function (Jensen, 1986). Using Eq. III-13 we see that $\mathrm{L}_{\mathrm{C}}<\mathrm{V}_{\mathrm{DP}}$ for $0<\mathrm{V}_{\mathrm{DP}}<0.9$. Since $\mathrm{L}_{\mathrm{C}}$ and $\mathrm{V}_{\mathrm{DP}}$ have different scales, $s_{L}$ and $s_{V}$ also have different scales.

Small errors in $s_{L}$ and $s_{V}$ can be "reconciled" in the following manner. To convert $s_{L}$ to an equivalent error in $V_{D P}$ divide $s_{L}$ by the derivative of Eq. III-3:

$$
\frac{\mathrm{dL}_{\mathrm{C}}}{\mathrm{dV} \mathrm{DP}_{\mathrm{P}}}=\exp \left(-\left[-0.5 \ln \left(1-\mathrm{V}_{\mathrm{DP}}\right)\right]^{2}\right) /\left[\sqrt{\pi}\left(1-\mathrm{V}_{\mathrm{DP}}\right)\right]
$$

Using this approach, the Lorenz estimator is usually more effictent than the Dykstra-Parsons estimator, with the former requiring only one-fourth the number of points needed by the latter for low $\left(\mathrm{V}_{\mathrm{DP}}<\right.$ 0.5) heterogeneity cases. As the heterogeneity increases, however, the Lorenz estimator efficiency decreases unt11, at $V_{D P}=0.9$, its efficiency is about equal to that of the Dykstra-Parsons estimator.

The Influence of Estimator Errors on Performance - A number of studies have related the performance of a particular reservoir model to the level of heterogeneity (Dykstra and Parsons, 1950; Schmalz and Rahme, 1950; Warren and Cosgrove, 1964; Koval, 1963; Craig, 1971; Khan, 1971; Arya et al., 1985). By consideration of some examples, the impact that estimator errors may have on reservoir performance can be demonstrated.

Koval (1963) discusses the influence of heterogeneity upon unstable miscible displacements. His figure 20 relates $V_{D P}$ to $H$, a heterogeneity factor, which enters into a miscible fractional flow equation. For a data set of 50 samples from a reservoir having $\mathrm{V}_{\mathrm{DP}}=$ $0.50(H=2.9), \quad s_{V}=0.073$ by Eq. (III-12). Hence, one could expect 
that $0.43 \leqq \hat{V}_{D P} \leqq 0.57$ for 67 percent of all possible data sets, or $0.35 \leqq \hat{V}_{D P} \leqq 0.65$ for 95 percent of such data sets. For the 67 percent confidence limits, $2.6 \leqq \hat{H} \leqq 3.4$, a 27 percent spread in $H$. For the 95 percent confidence limits, $2.3 \leqq \hat{H} \leqq 3.8$, a 50 percent spread in $H$. A unft mobilfty displacement requires $1 / H$ pore volumes injection for solvent breakthrough. Hence the 67 percent limits give a 0.09 pore volume uncertainty in the injected volume while the 95 percent limits give a 0.17 pore volume uncertainty.

Craig's monograph (1971) discusses the influence of heterogeneity upon simulated waterflood performance. A data set of 40 samples from a reservoir with $\mathrm{L}_{\mathrm{C}}=0.60$ gives $\mathrm{s}_{\mathrm{L}}=0.07$ or, ignoring the issue of bias, $0.53 \leqq \mathrm{~L}_{\mathrm{C}} \leqq 0.67$ for the 67 percent confidence 1imits. This implies that using the Stiles model, the amount of recovered oil may vary between 25 and 40 percent of the o11 recoverable at a 50 percent water cut. There is a one-in-three chance that the predicted recovery will fall outside of these limits when only 40 samples are used. Including the bias, $0.50 \leqq \hat{\mathrm{L}}_{\mathrm{C}} \leqq 0.64$, which gives a recovery between 28 and 44 percent at the 50 percent water cut.

Finally, the uncertainty in $\hat{\mathrm{V}}_{\mathrm{DP}}$ may be important when comparing estimates from different methods for a reservoir. Differences between $\hat{\mathrm{V}}_{D P}$ using core data and $\hat{\mathrm{V}}_{D P}$ based on transient tests have been reported (Warren, 1961). These differences are usually attributed to the scale of measurement. However, transient test data are usually few in number so that the variability associated with the estimate may be quite large. Hence, caution is required when comparing such estimates and drawing conclusions. 
Permeability Distribution Effects on Heterogenefty Measures - Several studies (Lambert, 1981; Goggin et al., 1986; Jensen et a1., 1985) have shown that permeability may not necessarily be log-normally distributed. In particular, the previous section has shown that permeability may be $p$-normally distributed. That is, $(k)^{p}$ is normally distributed for some value of $p,-1 \leqq p \leqq+1$, where $k$ is the permeability random variable. It is convenient mathematically to consider that the quantity $x$, defined as in Eq. (III-3), is $N\left(m_{x}\right.$, $s_{x}{ }^{2}$ ). Recall that the cases $p=0$ and $p=1$ indicate that the permeability is log-normally distributed or normally distributed, respectively.

We consider the behavior of $V_{D P}$ and $L_{C}$ when the permeability p.d.f. is p-normal. In doing so, we include two important cases, $p=$ 0 and $p=1$, but we also allow for a behavior that does not correspond to these two cases.

We present briefly some observations concerning p-normal distributions. See (Jensen, 1986) for a more extensive discussion. The first moment (i.e. the arithmetic mean) of a p-normal distribution only exists when $\mathrm{p} \geqq 0$. The geometric mean of a p-normal distribution always exists, as do the median and other percentiles.

The ratio $c_{x}=s_{x} / m_{x}$ and the exponent $p$ constitute a useful measure of the variability of a distribution; they allow two different distributions to be compared. The variable $c_{x}$ is a measure of how variable the permeability is, but gives no indication of how frequently high permeability values occur relative to the low permeability values. The exponent $p$ is a measure of how asymmetric the distribution is; the lower the value of $p$ the longer will be the 
right tail of the p.d.f. (Jensen, 1986). That is, as p decreases, there is an increasing probability that high (relative to the average permeability regions appear in the reservoir.

Permeability Distributions and the Lorenz Coefficient - To create a Lorenz plot from data, no knowledge of the permeability p.d.f. is required. However, we are seeking an $\mathrm{L}_{C}$ which is representative of the population and not just a value for the data at hand $\mathrm{L}_{\mathrm{C}}$ is a one-parameter assessment of the variability, or heterogeneity, present in the reservoir. As such, it has several limitations and a knowledge of the permeability p.d.f. can help us to interpret the estimate obtained.

The Lorenz plot assumes that an arithmetic mean value exists for the reservoir population (Gail and Gastwirth, 1978). For the cases -1 $\leqq p<0$, the arithmetic mean does not exist and, hence, the Lorenz coefficient does not exist. Consequently, for reservoirs where $p<0$, estimating a Lorenz coefficient is not useful. When $p \geqq 0$, a unique value of $L_{C}$ may be associated with each value of $c_{x}$ and $p$ (Jensen, 1986). Figure III-19 shows this relationship for the case of a 1000 md median permeability (A fixed median was chosen to keep all the distributions comparable.) However, for a given value of $\mathrm{L}_{\mathrm{C}}$, the values of $c_{x}$ and $p$ are not uniquely determined. For a fixed value of $c_{x}, L_{C}$ increases as $p$ decreases. This behavior implies that permeability distributions with small $\mathrm{p}$ values are potentially more heterogeneous than are distributions with large p values. That is, a 


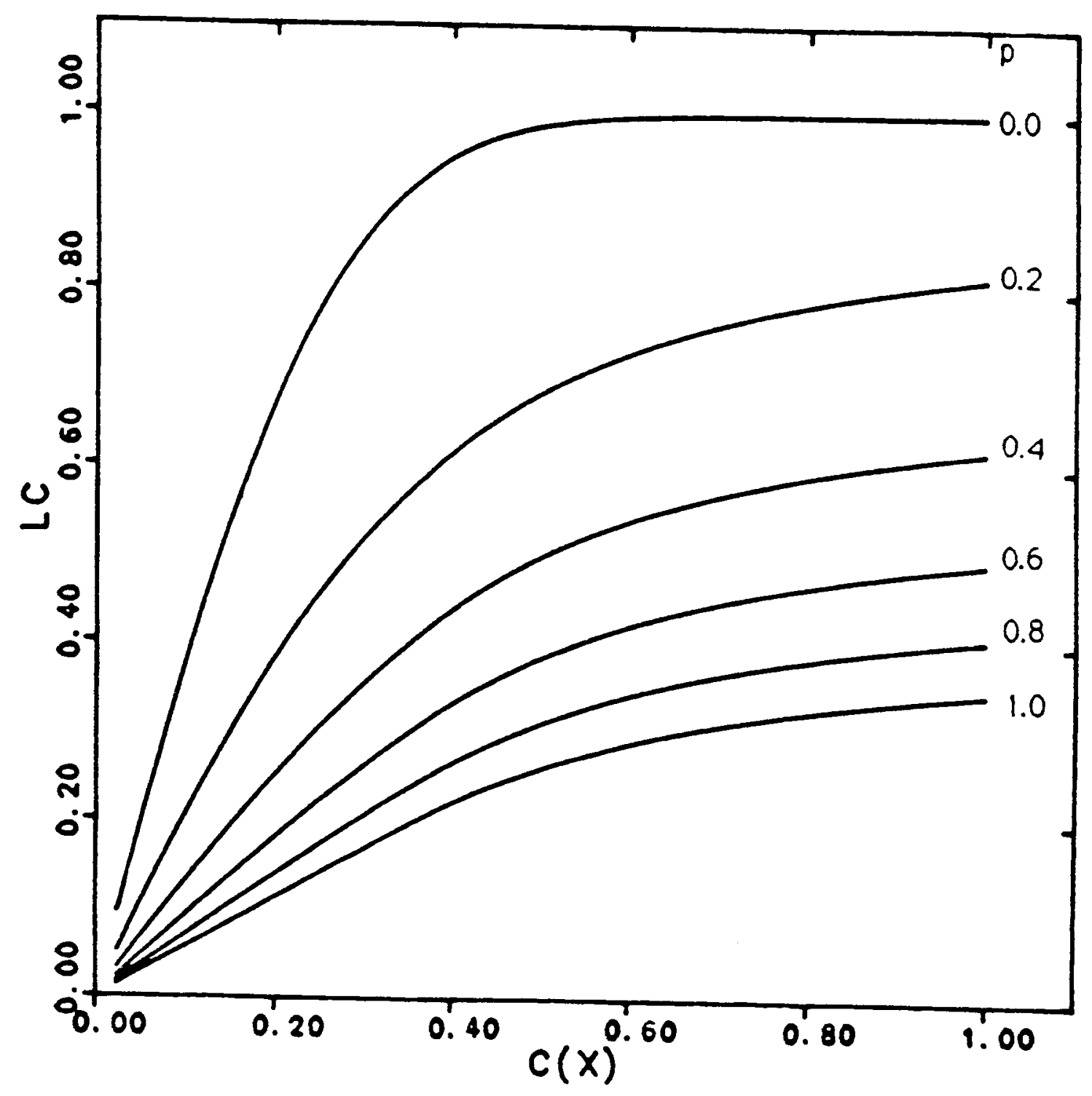

Figure III-19. Lorenz coefficients for populations having 1,000-md median permeability. 
p.d.f. with a small $p$ value may represent greater heterogeneity, depending upon the value of $c_{x}$.

Permeability Distributions and the Dykstra-Parsons Coefficient - The procedure for estimation of $V_{D P}$ calls for a log-normal probability plot to be made from the data (Dykstra and Parsons, 1950). The estimate $V_{D P}$ is based upon a "best-fit" line to the plot and, therefore, does not strictly require that the data come from a log-normal population. The line is used to estimate the median permeability, $k_{50}$, and the permeability at the $16^{\text {th }}$ percentile, $k_{16}$ to give

$$
\hat{\mathrm{V}}_{\mathrm{DP}}=1-\left(\hat{\mathrm{k}}_{16} / \hat{\mathrm{k}}_{50}\right)
$$

The use of a best-fit line makes this procedure difficult to quantify when $p \neq 0$, however a few qualitative remarks may be made.

If we ignore sampling error, a p-normally distributed variable will cause the log-normal probability plot to be concave upward or downward, depending upon whether $\mathrm{p}<0$ or $\mathrm{p}>0$, respectively. Since the best-fit line procedure calls for weighting the central portion of the plot more than the ends, the estimated median permeability, $\hat{k}_{50}$, will be close in value to the true median, $k_{50}$ (Fig. III-20). The estimate $\hat{k}_{16}$, however, will be influenced more: if $p>0$, then $\hat{k}_{16}>$ $\mathrm{k}_{16}$ (Fig. III-20); if $\mathrm{p}<0$, then $\hat{\mathrm{k}}_{16}<\mathrm{k}_{16}$. Hence, from Eq. (III-15), if $\mathrm{p}>0$, then $\hat{\mathrm{V}}_{\mathrm{DP}}<\mathrm{V}_{\mathrm{DP}}$ if $\mathrm{p}<0$, then $\hat{\mathrm{V}}_{\mathrm{DP}}>\mathrm{V}_{\mathrm{DP}}$. 


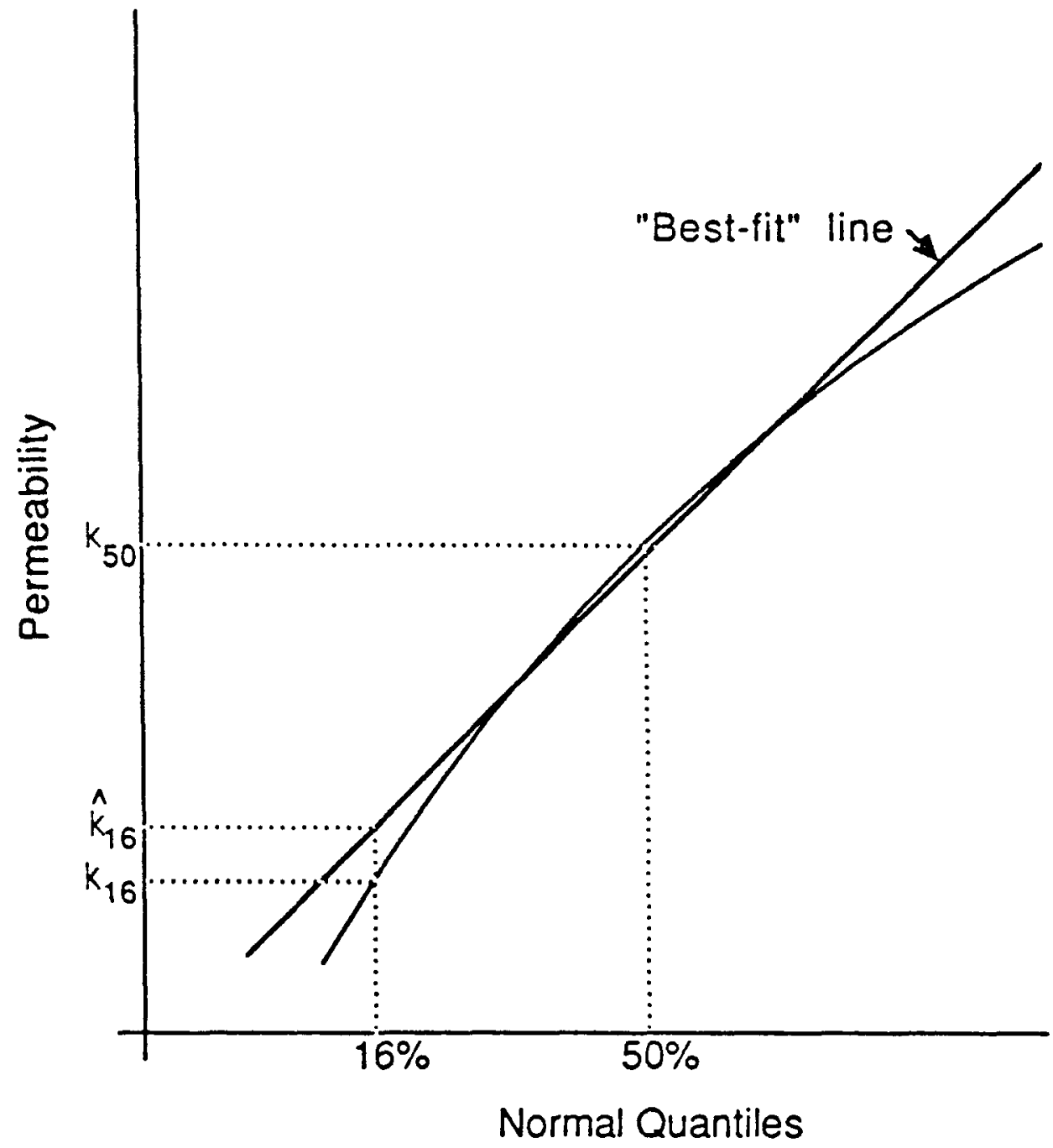

Figure III-20. Hypotheical Dykstra-Parsons plot for $\mathrm{p}>0$. 
When the best-fit line portion of the procedure is discarded and the $16^{\text {th }}$ and $50^{\text {th }}$ percentiles are used to estimate $V_{D P}$ directly from the data, some further results may be obtained. For the p-normal distribution (Jensen, 1986),

$$
k_{50}=\left(1+p \cdot m_{x}\right)^{1 / p}
$$

and

$$
k_{16}=\left[1+p\left(m_{x}-s_{x}\right)\right]^{1-p}
$$

Combining Eqs. (III-15) - (III-17) gives

$$
\mathrm{v}_{\mathrm{DP}}=1-\left[1-\mathrm{p} \cdot \mathrm{s}_{\mathrm{x}} /\left(1+\mathrm{p} \cdot \mathrm{m}_{\mathrm{x}}\right)\right]^{1 / \mathrm{p}}
$$

Thus, as is the case for the Lorenz coefficient, $V_{D P}$ does not uniquely define $p$ and $c_{x}$.

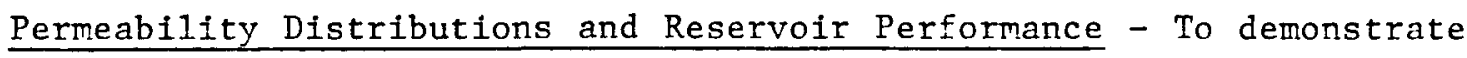
the influence that the permeability p.d.f. may have on reservoir performance, we created nine sets of permeability values which were divided into three groups. Table III-4 shows the features of the sets. Each group has three data sets which all have the same $V_{D P}$ but different exponent ( $p$ ) values. The permeability sets were then used in a Dykstra-Parsons layered mode (Dykstra and Parsons, 1950) to examine the variation in the water-oil ratio behavior from set to set. All data sets were constructed such that the median permeability value, $k_{50}$ was $500 \mathrm{md}$. Equation (III-16) then yielded $\mathrm{m}_{\mathrm{x}}$, since the $\mathrm{V}_{\mathrm{DP}}$ and $\mathrm{p}$ values were already chosen, and Eq. (III-18) was solved for 
TABLE III-4. Properties of data sets for reservoir simulations.

\begin{tabular}{|c|c|c|c|c|c|}
\hline Set No. & Group No. & $\mathrm{V}_{\mathrm{DP}}$ & $p$ & $m_{x}$ & $\mathrm{~s}_{\mathrm{x}}$ \\
\hline 1 & 1 & 0.25 & 0.0 & 6.21 & 0.29 \\
\hline 2 & 1 & 0.25 & 0.2 & 12.3 & 0.97 \\
\hline 3 & 1 & 0.25 & 0.4 & 27.5 & 3.26 \\
\hline 4 & 2 & 0.50 & 0.0 & 6.21 & 0.69 \\
\hline 5 & 2 & 0.50 & 0.2 & 12.3 & 2.24 \\
\hline 6 & 2 & 0.50 & 0.4 & 27.5 & 7.27 \\
\hline 7 & 3 & 0.75 & 0.0 & 6.21 & 1.39 \\
\hline 8 & 3 & 0.75 & 0.2 & 12.3 & 4.20 \\
\hline 9 & 3 & 0.75 & 0.4 & 27.5 & 12.8 \\
\hline
\end{tabular}


$\mathrm{s}_{\mathrm{x}}$. These values are shown on Table III-4. For each data set of $\mathrm{n}$ points, the $i^{\text {th }}$ datum, $k_{(i)}$, was computed as follows.

$$
\begin{aligned}
& x_{i}=m_{x}+\sqrt{2} \cdot s_{x} \cdot \operatorname{erf}^{-1}[(2 i-1) / n-1] \\
& k(i)=\left\{\begin{array}{l}
\left(1+p \cdot x_{i}\right)^{1 / p}, p \neq 0 \\
\exp \left(x_{i}\right), p=0
\end{array}\right.
\end{aligned}
$$

where erf ${ }^{-1}$ is the inverse error function and $i=1,2, \ldots \mathrm{n}$. Equation (III-20) assumes that the $i^{\text {th }}$ point has the associated probability $\left(\mathrm{k}^{-1 / 2}\right) / \mathrm{n}$.

Figures III-21 through III-24 show log-normal probability plots for the data sets for which $\mathrm{V}_{\mathrm{DP}}=0.5$ or $\mathrm{V}_{\mathrm{DP}}=0.75$ and $\mathrm{p}=0.2$ or $\mathrm{p}=$ 0.4. Each abcissa is scaled in units of standard deviations for the normal distribution. Each ordinate (y) is normalized by the mean and standard deviation of the data set:

$$
y_{i}=\left[\ln \left(k_{i}\right)-m\right] /(\sqrt{2} . s),
$$

where

$$
\begin{aligned}
& m=N-1 \Sigma i n\left(k_{i}\right), \\
& s^{2}=(N-1)^{-1} \Sigma\left[\ln \left(k_{i}\right)-m\right]^{2},
\end{aligned}
$$

and the summations are taken from $i=1$ to $i=N$. The values of $m$ and s are noted on each figure. See Jensen (1986) for more details of how these plots are generated. As expected, all plots are curved downward. The straight line on each plot gives the position that the 


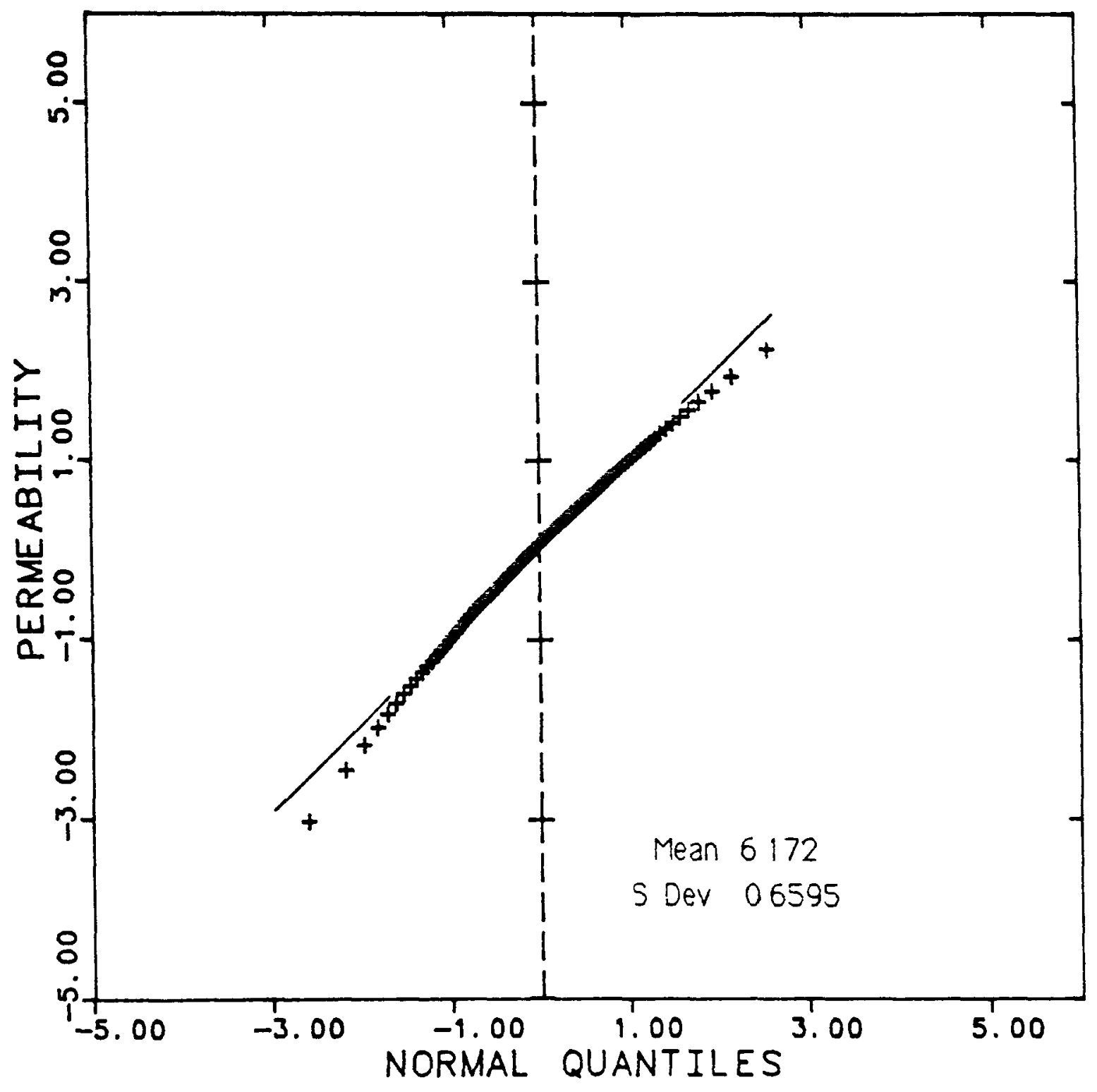

Figure III-21. Log-normal probability plot for data set No. 5 . 


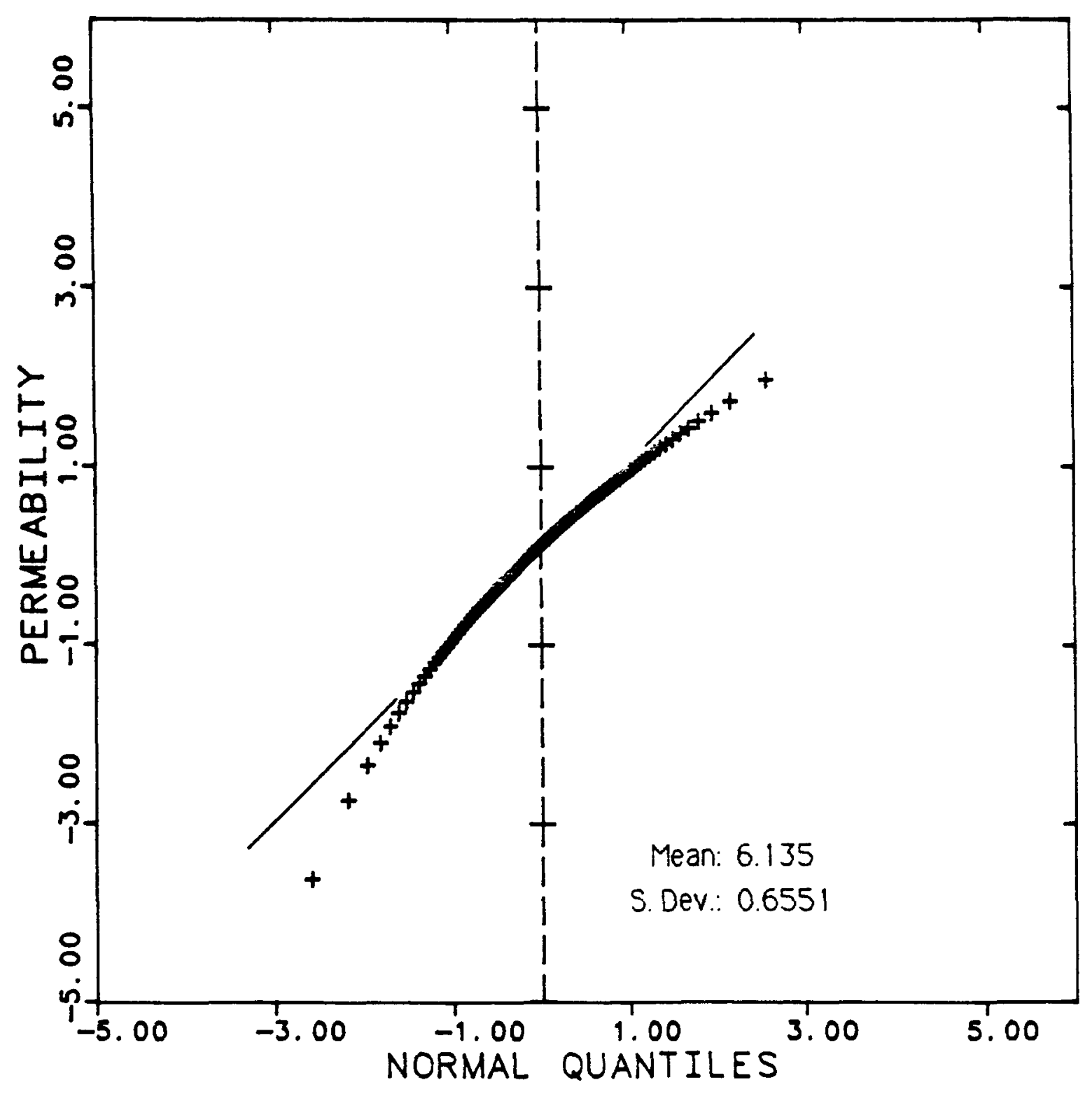

Figure III-22. Log-normal probability plot for data set No. 6 


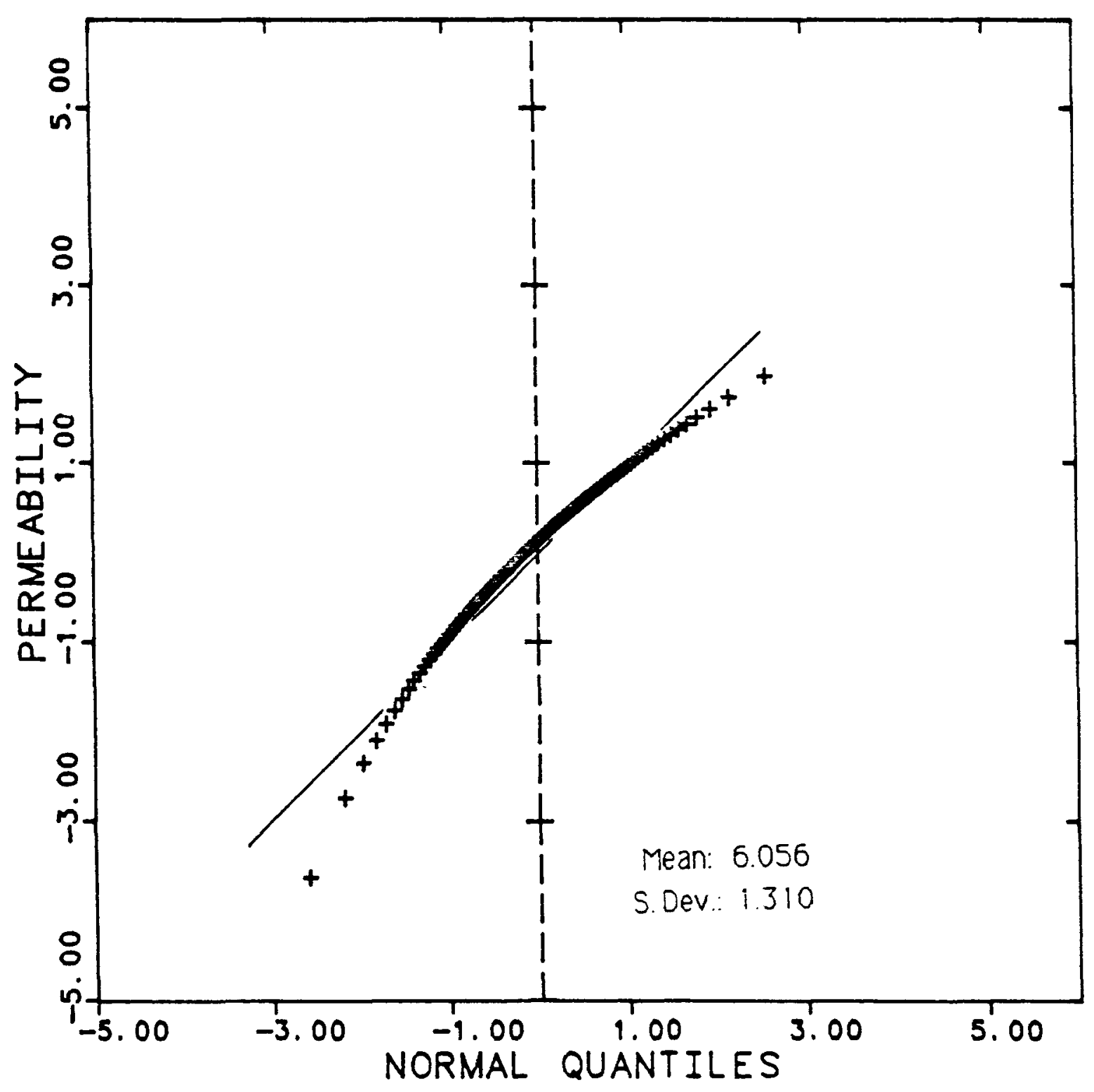

Figure III-23. Log-normal Probability Plot for Data Set No. 8. 


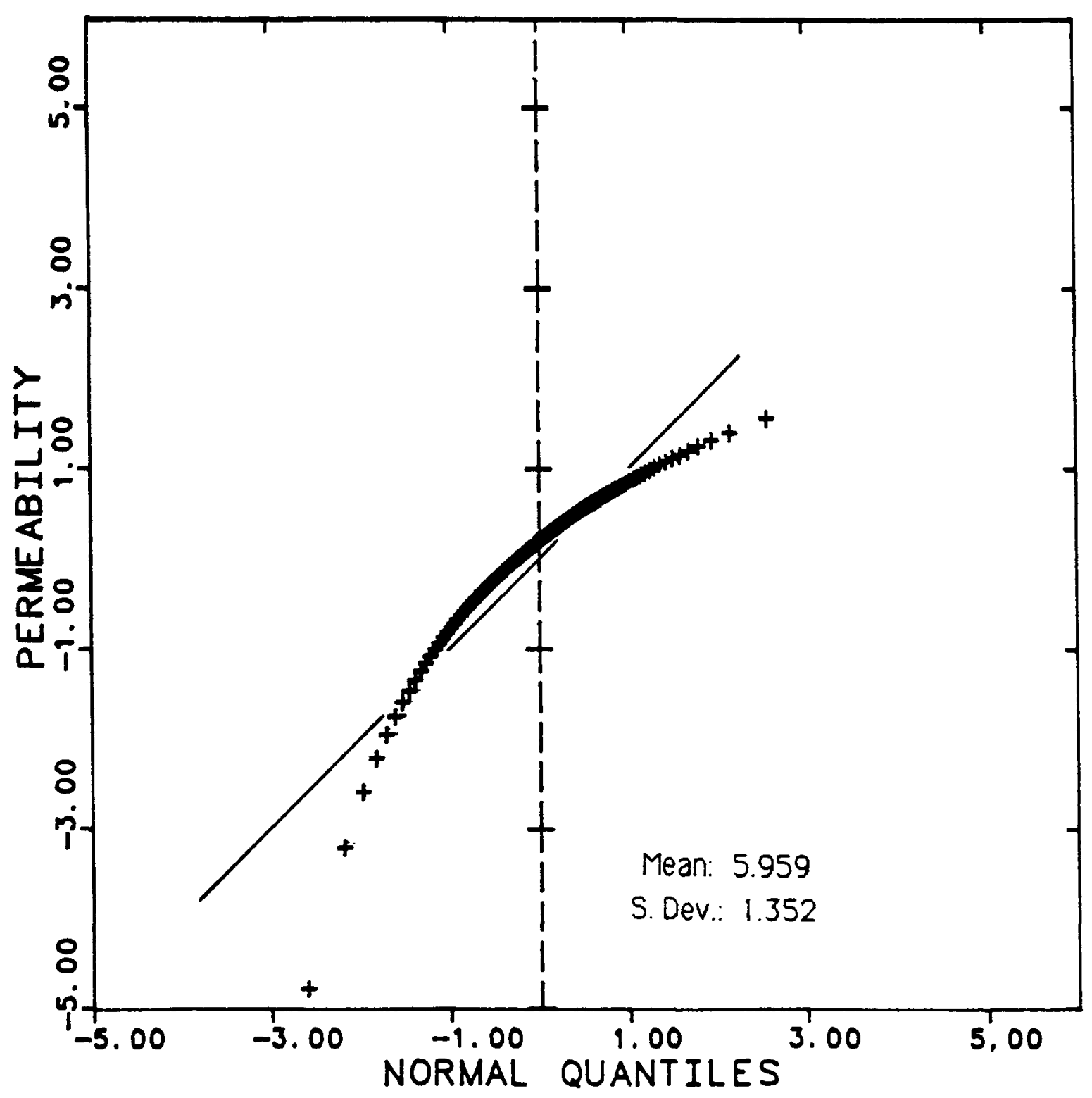

Figure III-24. Log-normal probability plot for data set No. 9. 
plot would have if the data were log-normally distributed with the stated $\mathrm{V}_{\mathrm{DP}}$. In terms of the best-fit line procedure called for by Dykstra and Parsons (1950), with weighting the center more than the outside points, the data calculated do have the desired coefficients. The data sets were used in a non-communicating layered reservoir model as described in Dykstra and Parsons (1950). Gravity and capillary effects are negligible, and the displacement is locally piston-like. Two mobility ratios, 0.5 and 2.0 , were considered. Figures III-25 through III-28 show the model behavior in terms of oil produced (in pore volumes) versus the water-oil ratio for the cases $V_{D P}=0.50$ and $\mathrm{V}_{\mathrm{DP}}=0.75$.

For the sets with $V_{D P}=0.25$, there was only a small difference in the model behavior as $p$ changed. At higher levels of heterogeneity, however, the influence of $\mathrm{p}$ was evident. For example, when $V_{D P}=0.75$, a log-normal distribution and a favorable mobility ratio $(M=0.5)$ behaves similarly to a 0.4 -normal distribution with an unfavorable mobility ratio $(M=2,0)$. For a unit water-oil ratio and $V_{D P}=0.75$, a change of exponent from $p=0$ to $p=0.2$ implies an increase of 0.15 pore volumes of oil recovered. These results indicate that $p$ may be as influential as $M$ in determining oil recovery.

The above analysis, while spectfic to $\mathrm{V}_{\mathrm{DP}}$, also indicates the results that would be observed with the Lorenz coefficient. Figure III-19 shows that an infinite number of $\left(c_{x}, p\right)$ pairs may be chosen to give the same $\mathrm{L}_{\mathrm{C}}$ value. Those pairs with low $\mathrm{p}$ values, however, would represent reservoirs with higher water cuts.

We conclude from these results that reservoirs with different 


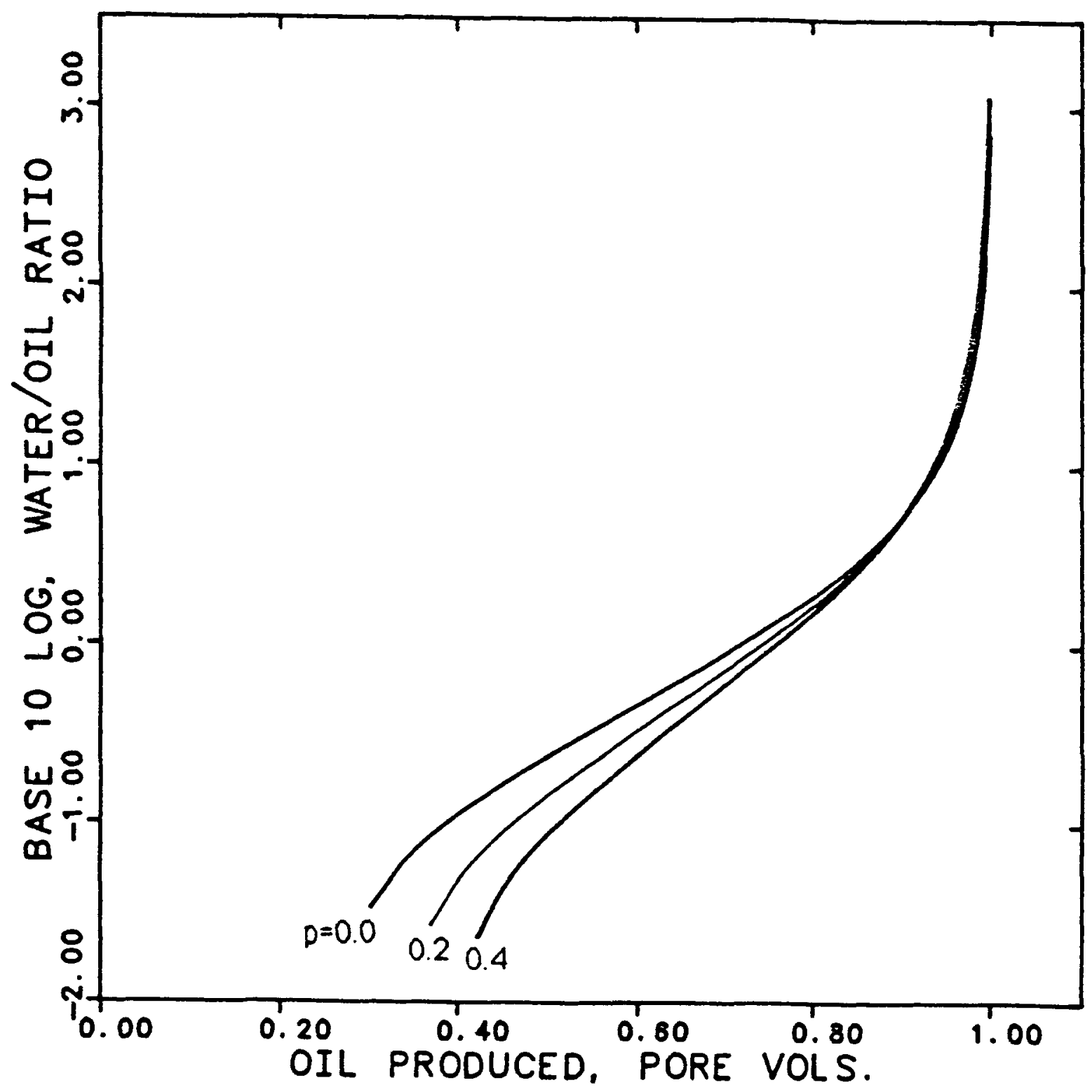

Figure III-25. Layered model behavior for $M=0.5$ and $V_{D P}=0.50$. 


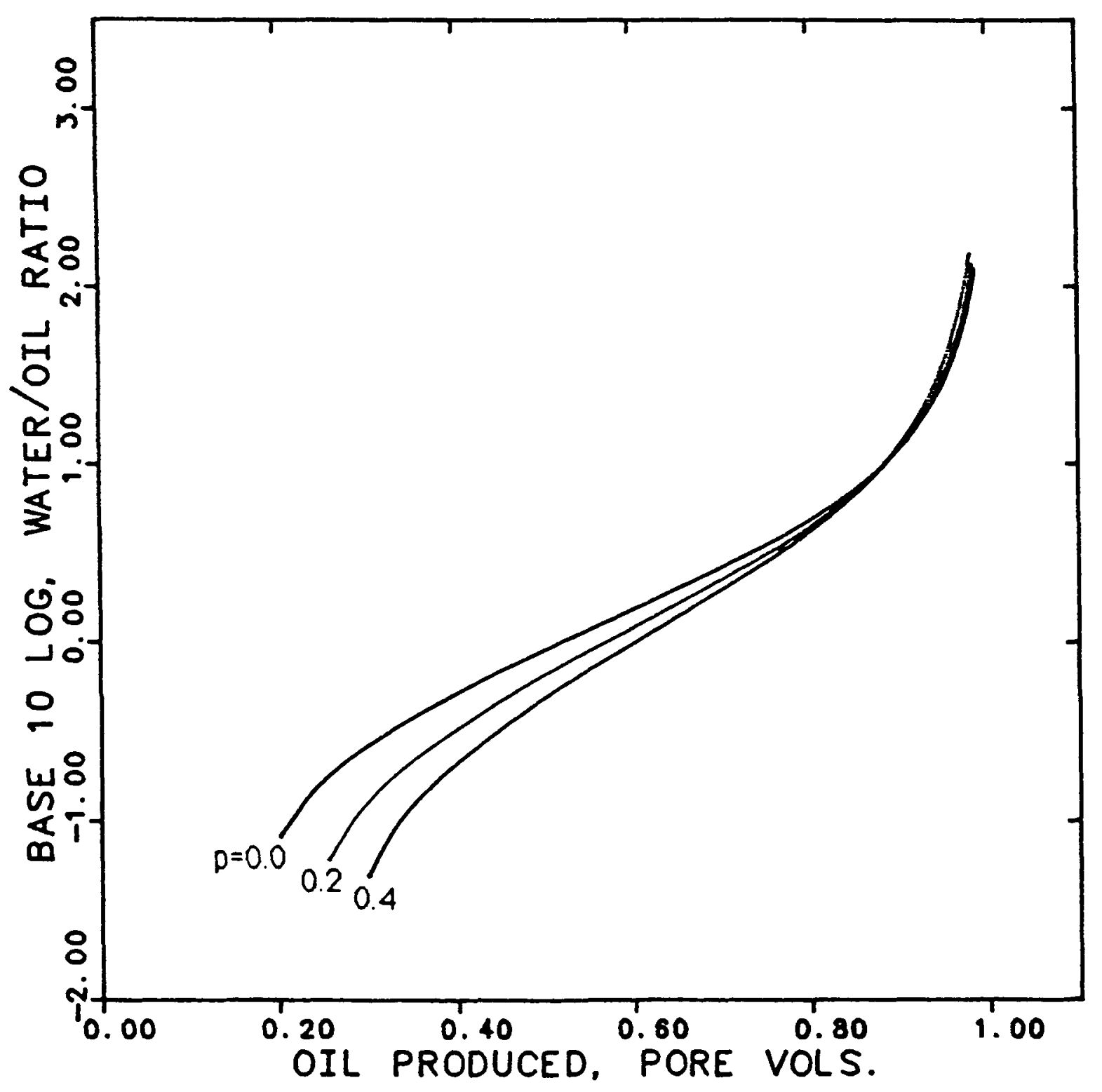

Figure III-26. Layered model behavior for $M=2.0$ and $V_{D P}=0.50$. 


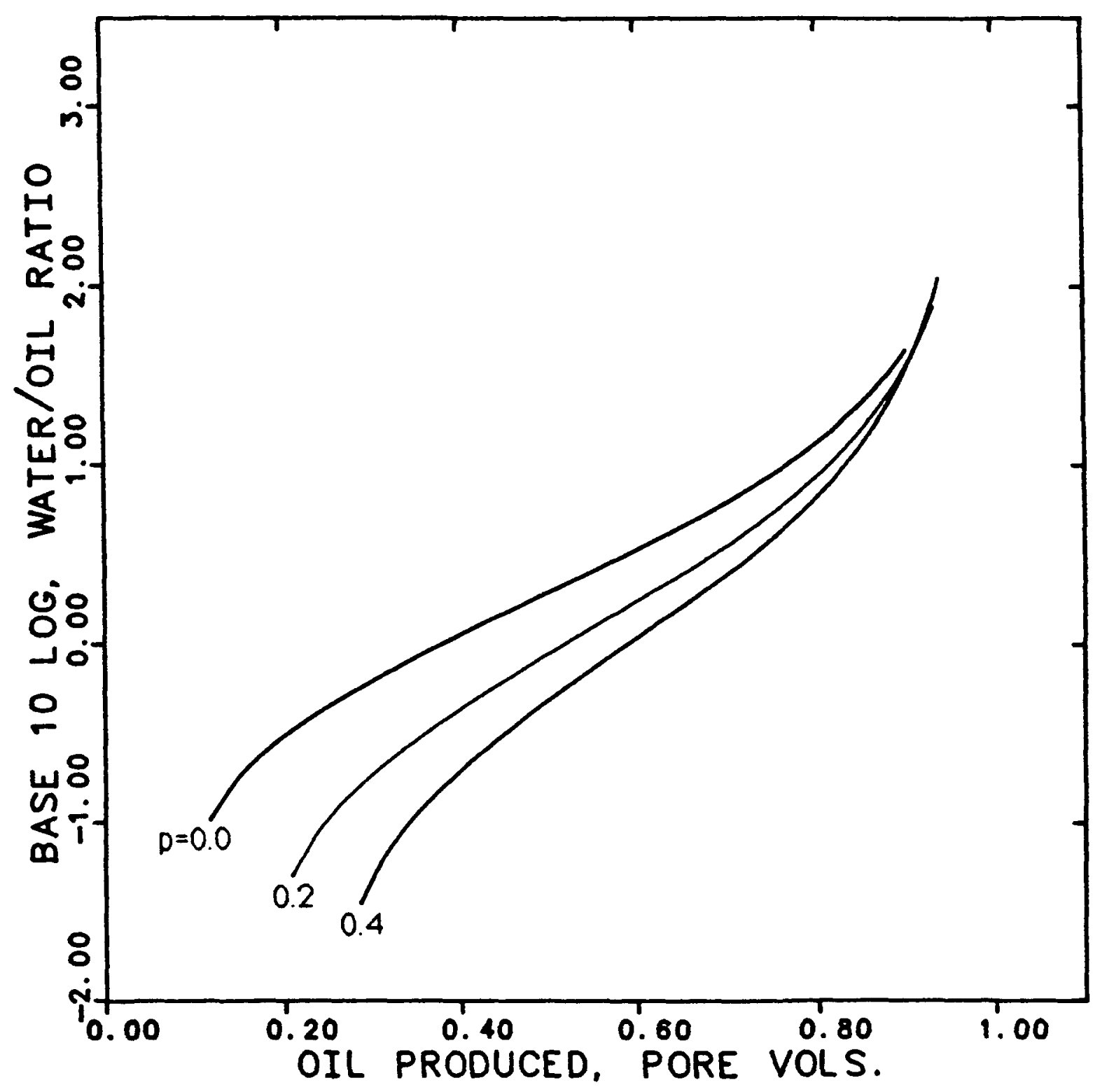

Figure III-27. Layered model behavior for $M=0.5$ and $V_{D P}=0.75$. 


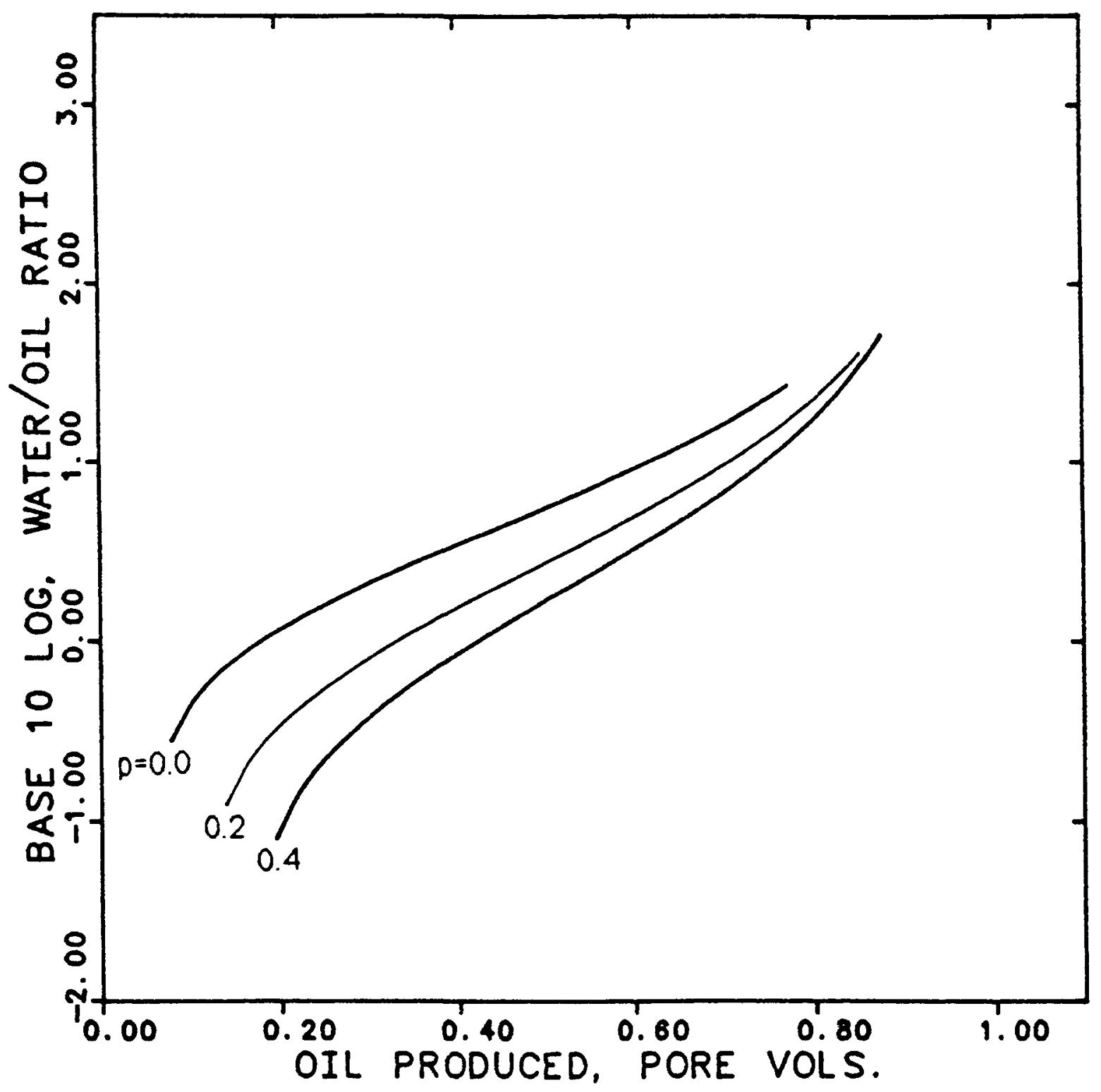

Figure III-28. Layered model behavior for $M=2.0$ and $V_{D P}=0.75$. 
permeability distributions but equal heterogeneity measures may occur. Information exists within data sets which is not normally used by the existing measures. The performance of reservoirs may vary considerably according to the permeability p.d.f.

A New Heterogeneity Measure - From an analysis of the preceding results, we propose a new heterogeneity measure. It has a mathematical form similar to the $V_{D P}$ estimator, but it does not require a plot. An example of the relative performances of the new measure, $\mathrm{V}_{\mathrm{DP}}$, and $\mathrm{L}_{\mathrm{C}}$ is given.

Desirable properties of a heterogeneity measure - The nature of a heterogeneity measure, however defined, is to distill in a meaningful way, the level of permeability variability in a reservoir. The computation of such a measure inevitably depends on data from a Iimited number of reservoir samples. Therefore, it should extract as much information as possible from those data.

On the basis of the preceding analysis of the traditional heterogeneity measures, several criteria for estimators are suggested. 1. The estimator should be unbiased.

2. The estimator should be efficient.

3. A measure of the permeability p.d.f. asymmetry (e.g. the exponent p) should be made.

4. The estimator should have a range of values which allows the higher heterogeneity cases to be readily distinguished.

5. The estimation technique should be easy to use and should avoid graphical methods. 
Neither the $V_{D P}$ nor the $L_{C}$ estimator satisfies these requirements. The issue of scale is particularly important because of the sampling problem: The zero-to-one scale of these estimators compresses the high heterogeneity cases together and the sampling variability blurs the distinction further.

To suggest a new measure, however, requires some caution. A substantial amount of work relating $V_{D P}$ or $L_{C}$ to the performance of reservoir models is available. Any new estimator should give results that can be interpreted in terms of $\mathrm{V}_{\mathrm{DP}}$ or $\mathrm{L}_{\mathrm{C}}$.

A new heterogeneity measure - We propose that the pair of values, $V$ and $p$, where

$$
\mathrm{V}=\mathrm{k}_{84} / \mathrm{k}_{16}-1
$$

be used to assess permeability heterogeneity. The form of Eq. (III-22) is similar to the $\mathrm{V}_{\mathrm{DP}}$ estimator. The coefficient $\mathrm{V}$ varies between zero and infinity with, as for the traditional measures, zero representing a homogeneous reservoir.

The estimation of the required quantities is straightforward. The exponent $p$ is readily estimated from a data set by one of the methods discussed above. The quantities $k_{16}$ and $k_{84}$ may be estimated from a set of $n$ data by the following formulas (Hoaglin et a1., 1983)

$$
k_{16}=k_{i}+\left(k_{i+1}\right)-k_{1} \cdot(r-1)
$$

and

$$
k_{84}=k_{j}+\left(k_{j+1}-k_{j}\right) \cdot(t-j)
$$


where

$$
\begin{aligned}
& r=0.1587(n+1 / 3)+1 / 3, \\
& t=0.8413(n+1 / 3)+1 / 3, \\
& i=[r], \quad j=[t],
\end{aligned}
$$

[z] denotes the largest integer $\leqq z$, and $k_{m}$ denotes the $m^{\text {th }}$ datum in the ordered set of permeability data, $k_{1} \leqq k_{2} \leqq k_{3} \leqq \ldots \leqq k_{N}$. These formulas determine the locations of the $16^{\text {th }}$ and $84^{\text {th }}$ percentiles for an arbitrary number of data. The estimation of $k_{16}$ and $k_{84}$ requires no fitting of lines to data, although plotting may be desirable for other reasons.

For the case of a log-normal distribution $(p=0)$, several results may be derived. The coefficients $\mathrm{V}, \mathrm{V}_{\mathrm{DP}}$, and $\mathrm{L}_{\mathrm{C}}$ are related by

$$
\mathrm{V}_{\mathrm{DP}}=1-(\mathrm{V}+1)^{1 / 2}
$$

and

$$
\mathrm{L}_{\mathrm{C}}=\operatorname{erf}[1 / 4 \cdot \ln (\mathrm{V}+1)]
$$

For example, when $\mathrm{V}_{\mathrm{DP}}=0.5, \mathrm{~V}=3.0$ and when $\mathrm{L}_{\mathrm{C}}=0.7, \mathrm{~V}=16$ Using the approach given in Appendix A, the bias, $\mathrm{m}_{v}$, and the standard error $s_{v}$, of the estimator for $v$ are given by

$$
\begin{aligned}
m_{v} & =[(1+v)]^{2}\left[0.463-0.570(1+v)^{-1 / 2}\right. \\
& \left.+-0.570(1+v)^{1 / 2}-0.108 V\right] / \mathrm{N}
\end{aligned}
$$




$$
\mathrm{m}_{\mathrm{v}}=0.962 \cdot(1+v) \cdot \ln (1+\mathrm{V}) / \sqrt{\mathrm{n}}
$$

An analysis of Eq. (III-23) shows that the bias is positive for $V$ $\leqq 35$ (i.e. $V_{D P} \leqq 0.83$ ) and is negative otherwise. This means that the $\mathrm{V}$ estimator tends to be slightly pessimistic ( $\mathrm{V}$ too high) for most reservoirs. Cases of very high heterogeneity $\left(\mathrm{V}>33\right.$ or $\left.\mathrm{V}_{\mathrm{DP}}>0.83\right)$ give a negative bias. If $\mathrm{N} \geqq 40$, then $\left|\mathrm{m}_{\mathrm{v}}\right| \leqq 0.05 \mathrm{~V}$ for $\mathrm{V} \leqq 225$ (i.e $\left.\mathrm{V}_{\mathrm{DP}} \leqq 0.93\right)$. Only in cases of exceptionally high heterogeneity $(\mathrm{V}>$ 400 or $V_{D P}>0.95$ ) would $N \geqq 50$ be required to keep the blas within five percent of $V$. A study of the standard error performance of the $V$ estimator, using the same approach as described for the comparison of $s_{L}$ and $s_{V}$, shows that $V$ is more efficient than $V_{D P}$ (Jensen, 1986). If, for a given situation, $n$ points are required to estimate $V_{D P}$ with standard error $\mathrm{s}_{\mathrm{V}}$, then $0.43 \mathrm{~N}$ points are required to estimate $\mathrm{V}$ to an equivalent standard error. These results are based on large-sample theory and, therefore, should be used with caution when $n<40$.

The Relative Performance of the Three Measures - We compared the overall performance of the $V, V_{D P}$ and $L_{C}$ estimators by determining the error of the fractional oil recovered $\left(E_{R}\right)$ in a Dykstra-Parsons Mode1 (Dykstra, Parsons, 1950). For the base case, we took $\mathrm{V}_{\mathrm{DP}}=0.80\left(\mathrm{~L}_{\mathrm{C}}=\right.$ 0.745 and $V=24), M=1$ and an Irreducible water saturation of 0.30 . Assuming a data set of a given size, six values of $E_{R}$ at a water-oil ratio of 100 were calculated based on the standard error range of each estimator: $V_{D P} \pm s_{V}, L_{C}+m_{L} \pm s_{L}$, and $V \pm s_{V}$. (The bias was included in the Jorenz estimate because of its appreciable value.) This was 
done for a range of data set sizes. A log-normal permeability distribution was assumed.

The results are shown in Fig. III-29. The base case gives $E_{\mathrm{R}}=$ 0.35 (zero error). For any estimator, the spread of points above ( $E_{R}$ $>0.35)$ and below $\left(E_{R}<0.35\right)$ the base case line is governed by segveral factors:

1. The standard error of the estimator,

2. the bias of the estimator (signigicant only for $\mathrm{L}_{\mathrm{C}}$ and

3. the relationship between the heterogeneity measure and reservoir behavior.

The three estimators perform similarly regarding recovery overestimates $\left(E_{R}>0.35\right)$, with $V$ performing slightly better than either $\mathrm{V}_{\mathrm{DP}}$ or $\mathrm{L}_{\mathrm{C}}$. The recovery underestimate behavior $\left(\mathrm{E}_{\mathrm{R}}<0.35\right)$, however, is distinctly different for each estimator. The influence of bias on $\mathrm{L}_{\mathrm{C}}$ is sufficient to give a constant standard error behavior. Both $\mathrm{L}_{\mathrm{C}}$ and $\mathrm{V}$ are much less pessimistic than $\mathrm{V}_{\mathrm{DP}}$. The high efficiency of the $\mathrm{L}_{\mathrm{C}}$ estimator, particularly at small sample sizes, makes it attractive for this particular case. The relatively symmetrical error behavior of the $V$ estimator is a result of its zero-to-infinity scale. This is in contrast to the asymmetrical behavior of $\mathrm{V}_{\mathrm{DP}}$ and $\mathrm{L}_{\mathrm{C}}-$ even at 100 samples where $m_{L}$ is small - cased by the zero-to-one scale.

Observations and Conclusions - The Dykstra-Parsons and Lorenz heterogeneity measures have been examined assuming a log-normal p.d.f. and found to have the following properties:

1. Both measures compress the high heterogeneity cases, which makes performance predictions sensitive to the precise value used. 


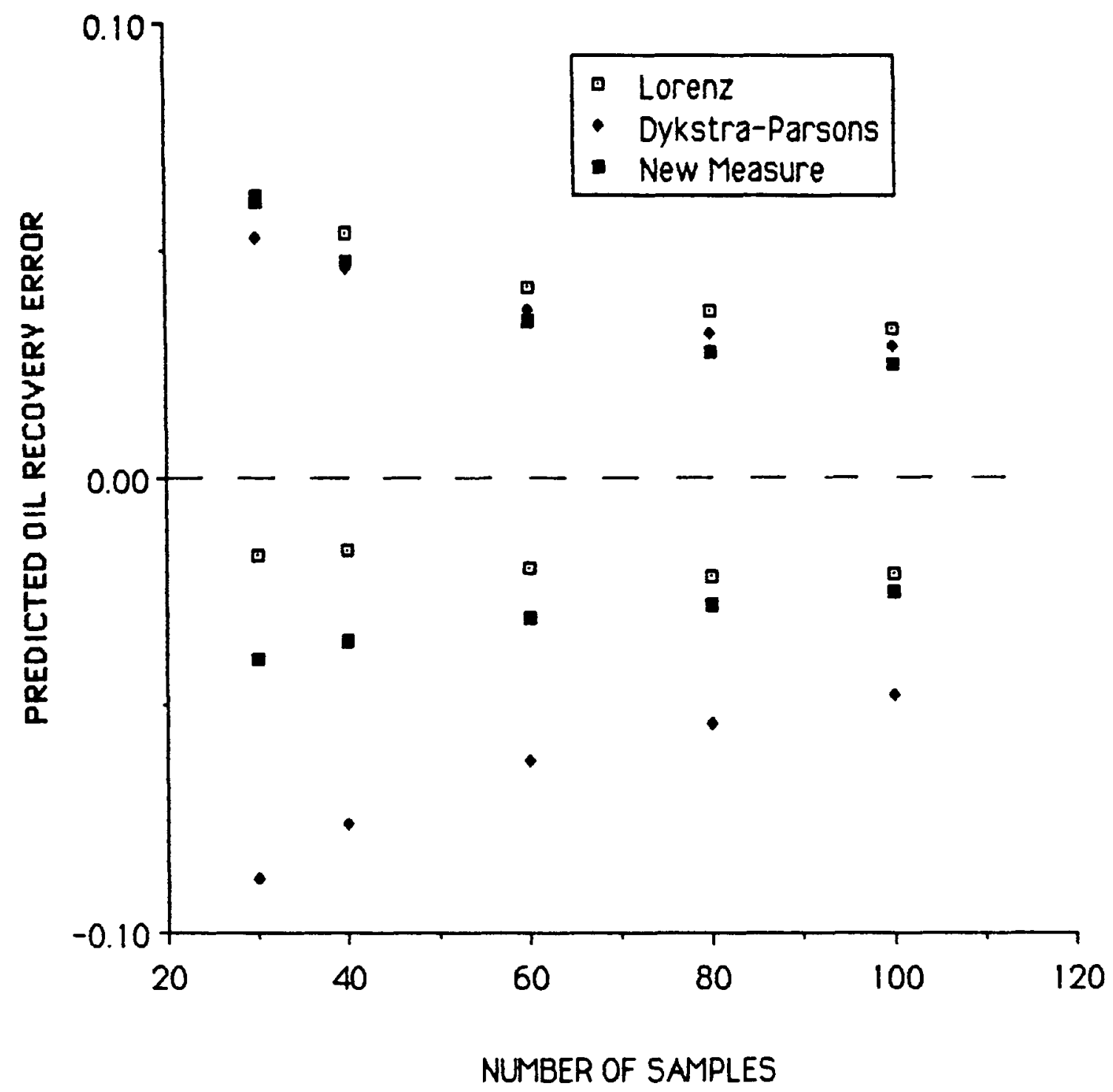

Figure III-29. Relative performance of heterogeneity measures for a layered model. 
2. Both estimators underestimate, on average, the true reservoir values. The Lorenz estimate is significantly biased for data sets having fewer than 40 samples.

3. The Lorenz estimator is much more efficient than the Dykstra-Parsons estimator in low heterogeneity cases. Both estimators are equally efficient for very high $\left(V_{D P} \geqq 0.9\right)$ heterogeneity cases.

4. Different reservoirs with the same $\mathrm{V}_{\mathrm{DP}}$ or $\mathrm{L}_{\mathrm{C}}$ may behave quite differently because of the asymmetry of the permeability distribution.

5. A Lorenz coefficient cannot be defined for some non-log-normal permeability distributions, $V_{D P}$, however, always exists.

A heterogeneity measure has been proposed which accounts for the permeability distribution. The measure does not require that a plot be made, but it does require that two parameters be estimated. For a log-normal p.d.f., the new measure is always more efficient than the Dykstra-Parsons estimator and it is more efficient than the Lorenz estimator in high heterogeneity $\left(V_{D P} \geqq 0.9\right)$ cases.

In comparison to the $\mathrm{V}_{\mathrm{DP}}$ and $\mathrm{L}_{\mathrm{C}}$ estimators, more computation is required to estimate $\mathrm{V}$ and $\mathrm{p}$. Information which may be important to performance prediction, however, is being lgnored when only $V_{D P}$ and $L_{C}$ is computed. In view of the cost of data, it should be worthwhile to make the added computational effort. 
We will use the turning bands method (TBM) in part $V$ to generate a stochastic field. The method employs a weighted sum of a number of one-dimensional Iine processes to generate a 2-dimensional field. The generation of a line process is considered here.

Let $z$ be the property (permeability here) to be generated.

$$
z(\vec{x})=\frac{1}{\sqrt{\bar{L}}} \sum_{i=1}^{L} z_{i}\left(\vec{x} \cdot \vec{u}_{i}\right)+\mu
$$

where $\vec{x}$ is the position vector, $\vec{u}_{i}$, is the unit vector along line $i$, $z_{i}$ is the corresponding value at line $i$ evaluated at $\vec{x}^{*} \vec{u}_{i}$ and $L$ is the number of lines uniformly extending from the origin (Montoglou and Wilson, 1982).

The square root on $L$ is in accordence with the law of addition of random functions. The theoretical mean of this process is zern if the Iine processes are generated with zero mean, therefore, the required mean value $\mu$ is added after generation.

The major assumptions in this method are that $z_{i}$ are secondary stationary (stationarity of order two), isotropic and normally distributed.

Line Process - The line process is generated using the modified spectral method (Shinozuka and Jan, 1972),

$$
z_{i}(x)=2 \sum_{k=1}^{m} A\left(\omega_{k}\right) \cos \left(\omega_{k}^{\prime} \cdot x+\phi_{k}\right)
$$


where

$$
\begin{aligned}
& \omega_{k}=\left(k-\frac{1}{2}\right) \Delta \omega \\
& \omega_{k}^{\prime}=\omega_{k}+\delta \omega \\
& A\left(\omega_{k}\right)=\sqrt{S\left(\omega_{k}\right) \Delta \omega} .
\end{aligned}
$$

$\phi_{\mathrm{k}}$ are $\mathrm{M}$ independent random phase angles uniformly distributed between 0 and $2 \pi$. $\delta \omega$ is a fine-tuning small angle to allow fewer terms in the sum of Eq. (III-25) (smaller $\mathrm{m}$ ). $\mathrm{S}$ is the one-dimensional spectral density function calculated from the given isotropic autocovariance function. $m$ should be large enough to achieve accuracy, and $m \Delta \omega$ should be large enough so that $\mathrm{A}(\mathrm{m} \Delta \omega)$ approaches zero (Montoglou and Wilson, 1982). Normally $\mathrm{m}=100$ is sufficient.

Correlogram and Estimate - An isotropic secondary stationary process is completely determined by the mean

$$
\mu=E[z(\vec{x})]
$$

and the autocovariance function

$$
C(h)=E[\{z(x)-\mu\}\{z(x+h)-\mu\}]
$$

where $h$ is the separation distance. If the autocovariance is normalized by the variance, the zero-lag value of the autocovariance function, it becomes the autocorrelation function

$$
\rho(h)=\frac{C(h)}{C(0)}
$$


In other words, if the standard deviation $\sigma=\sqrt{\mathrm{C}(0)}$ is unity, autocovariance equals autocorrelation. The autocorrelation function is a one-parameter special case of a correlation function. In the following discussion the standard deviation is assumed unity unless otherwise noted, and the term correlation function is thus used for both the autocorrelation and autocovariance functions.

When processing data to describe a set of observations, the correlogram, which is the correlation as a function of separation distance, can be plotted. If the true mean or the population mean $\mu$ is known, an estimate of the correlation function can be calculated

$$
C_{\mu}(h)=\frac{1}{N} \sum_{i=1}^{N}\left(z_{i}-\mu\right)\left(z_{i+k}-\mu\right)
$$

where $h$ is the separation distance between the correlated points at $x_{i}$ and $x_{i+k}$

$$
\mathrm{h}=\mathrm{k} \cdot \Delta \mathrm{x}
$$

assuming a uniform grid of size $\Delta x$ and

$$
z_{i}=z\left(x_{i}\right)
$$

However, the population mean $\mu$ is rarely available in characterizing a process, and we must use the sample mean

$$
\hat{\mu}=\frac{1}{N} \sum_{i=1}^{N} z_{i}
$$


as an estimate of the population mean $\mu$. The correlogram becomes

$$
C_{\hat{\mu}}(h)=\frac{1}{N} \sum_{i=1}^{N}\left(z_{i}-\hat{\mu}\right)\left(z_{i+k}-\hat{\mu}\right)
$$

$\mathrm{C}_{\hat{\mu}}$ is an estimate of $\mathrm{C}_{\mu}$.

$\mu$

Using the sample mean introduces error in the correlation function since the sample mean can be different from the population mean. We would like to know when and under what conditions this exror is important.

Deviation of correlogram because of the sample mean - Let the difference between the sample mean and the population mean be $d \hat{\mu}$

$$
\hat{d \mu}=\hat{\mu}-\mu
$$

then

$$
\begin{aligned}
& \left(z_{i}-\hat{\mu}\right)\left(z_{i+k}-\hat{\mu}\right)=\left(z_{i}-\mu-d \hat{\mu}\right)\left(z_{i+k}-\mu-\hat{d \mu}\right) \\
& \quad=\left(z_{i}-\mu\right)\left(z_{i+k}-\mu\right)+d \hat{\mu}^{2}-\hat{d \mu}\left(z_{i}-\mu+z_{i+k}-\mu\right)
\end{aligned}
$$

and the correlation function using the sample mean becomes

$$
\begin{aligned}
\mathrm{C}_{\hat{\mu}}(h) & =\frac{1}{N} \sum_{i=1}^{N}\left(z_{i}-\hat{\mu}\right)\left(z_{i+k}-\hat{\mu}\right) \\
& =\frac{1}{N} \sum_{i=1}^{N}\left(z_{i}-\mu\right)\left(z_{i+k}-\mu\right) \\
& +(\hat{\mu}-\mu)^{2}
\end{aligned}
$$




$$
\begin{aligned}
& -(\hat{\mu}-\mu) \frac{1}{N} \sum_{i=1}^{N}\left(z_{i}-\mu+z_{i+k}-\mu\right) \\
& =C_{\mu}(h)+(\hat{\mu}-\mu)\left\{(\hat{\mu}-\mu)-\left(\hat{\mu}_{i}-\mu\right)-\left(\hat{\mu}_{i+k}-\mu\right)\right\}
\end{aligned}
$$

where $\hat{\mu}_{i}$ is $z_{i}$ averaged from $i=1$ to $N$ and $\hat{\mu}_{i+k}$ from $i=1+k$ to $N+k$. For a stationary process, $E\left[\hat{\mu}_{i}\right]=E\left[\hat{\mu}_{i+k}\right]$. Therefore, take the expectation:

$$
E\left[C_{\hat{p}}(h)\right]=C_{\mu}(h)-\sigma_{\hat{p}}^{2}
$$

Since the variance of the sample mean $\sigma_{\rho}^{2}$ is always positive, $\mathrm{C}_{\mu}(\mathrm{h})$ is a biased (systematically smaller) estimate of $\mathrm{C}_{\mu}(\mathrm{h})$. We need to evaluate the variance of the sample mean $\sigma_{a}^{2}$ in order to predict the bias.

Variance of the sample mean - The sample mean $\hat{\rho}$ is an unbiased estimate of the population mean $\mu$ as its expectation. The variance is the second moment about the expectation,

$$
\begin{aligned}
\sigma_{\hat{p}}^{2} & =E\left[(\hat{\mu}-\mu)^{2}\right] \\
& =E\left[\left(\frac{1}{N} \sum_{i=1}^{N} z_{i}-\mu\right)^{2}\right] \\
& =E\left[\left(\frac{1}{N} \sum_{i=1}^{N}\left\{z_{i}-\mu\right\}\right)^{2}\right]
\end{aligned}
$$




$$
\begin{aligned}
& =E\left[\left(\frac{1}{N^{2}} \sum_{i=1}^{N}\left(z_{i}-\mu\right)^{2}\right]\right. \\
& +E\left[\left(\frac{2}{N^{2}} \sum_{j=1}^{N-1} \quad \sum_{i=1}^{N-j}\left(z_{i}-\mu\right)\left(z_{i+j}-\mu\right)\right]\right] \\
& \sigma_{\hat{\mu}}=\frac{\sigma^{2}}{N}+\frac{2}{N} \sum_{i=1}^{N-1} \frac{(N-i) C_{\mu}(h)}{N}
\end{aligned}
$$

where $\sigma$ is the population standard deviation and $h=i \cdot \Delta x$ is the lag distance. The first term is the same as for independent $z_{i}$. The second term is caused by the correlation between the data points and cannot be reduced by simply increasing $\mathrm{N}$.

Let us assume an exponential correlation function:

$$
C_{\mu}(h)=\sigma^{2} \exp (-h / \lambda)=\sigma^{2} \exp (-a \cdot i)
$$

where $\lambda$ is the correlation length and $a=\Delta x / \lambda$ is the grid size/correlation length. The variance of the mean $\sigma_{\hat{\rho}}^{2}$ can then be calculated for a different number of points $N$ and different length $a$. The result is

$$
\sigma_{a}^{2}=\frac{\sigma^{2}}{N}\left(1+2 \sum_{i=1}^{N-1} \frac{(N-i) \exp (-a \cdot i)}{N}\right)
$$

The standard deviation of the mean $\sigma_{\beta}$ (with $\sigma=1$ ) calculated using Eq. $(I I I-31)$ ) is plotted in Fig. III-30 versus $\mathrm{N} \cdot \mathrm{a}$ (the total sample span divided by correlation length, $N \Delta x / \lambda)$. Figure III-30 shows that when the total sample span is less than about 20 times the correlation 


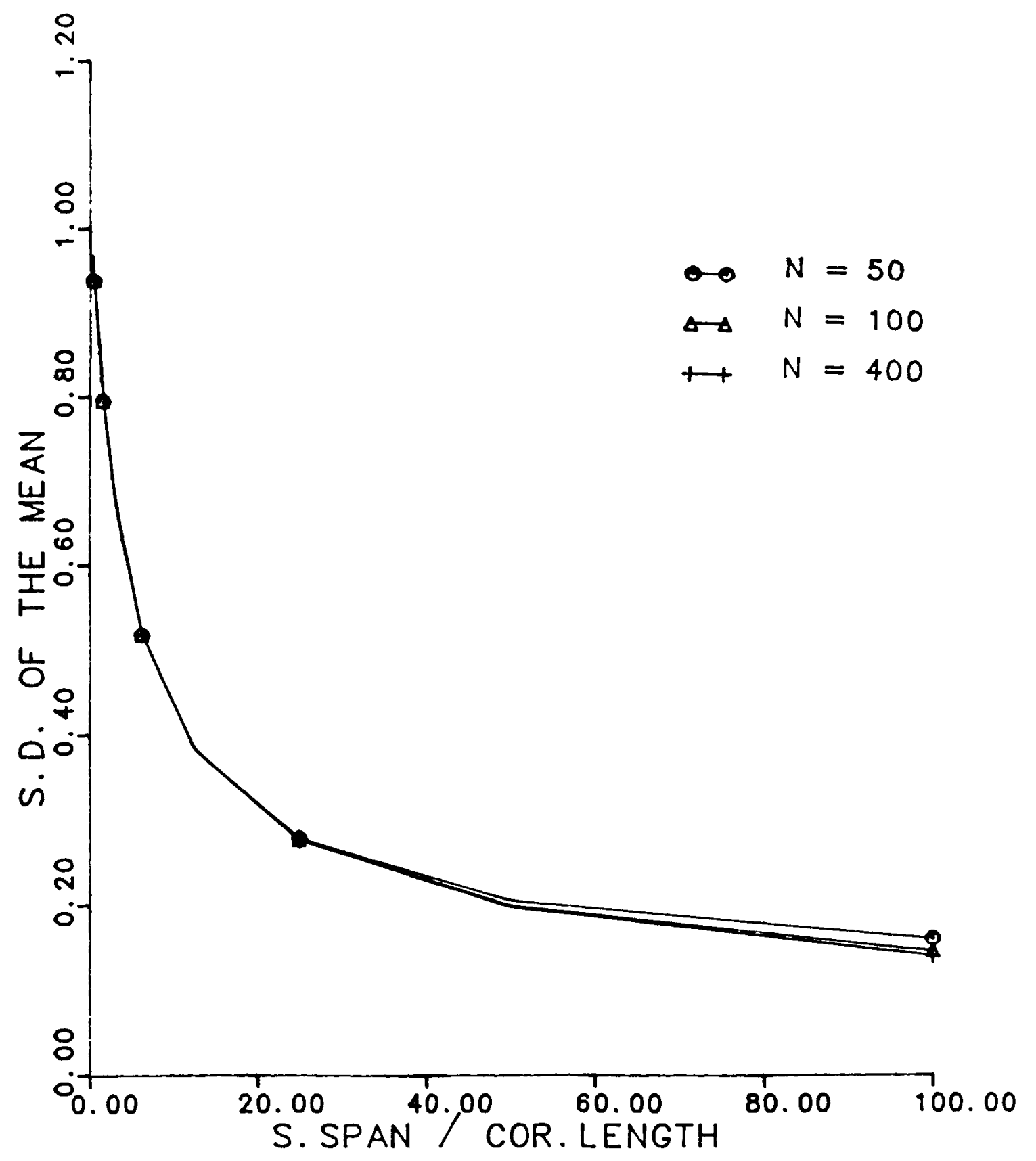

Figure III-30. Standard deviation of the mean. 
length, the standard deviation of the mean increases rapidly and approaches the unit population variance, i.e. $\sigma_{\hat{\rho}}^{2} \rightarrow \sigma^{2}$. An increase in the number of sample points $\mathrm{N}$ above a certain limit contributes little to a reduction in the standard deviation of the mean. This can be derived from Eq. (III-3I) by taking the limit $a=\Delta x / \lambda$ to zero

$$
\begin{aligned}
\lim _{a \rightarrow 0} \sigma_{p}^{2} & =\frac{\sigma^{2}}{N}\left(1+2 \sum_{i=1}^{N-1} \frac{(N-i) \exp (0 \cdot i)}{N}\right) \\
& =\frac{\sigma^{2}}{N}\left(1+2 \sum_{i=1}^{N-1} \frac{(N-i)}{N}\right) \\
& =\frac{\sigma^{2}}{N}\left(1+2 N-2-2 \sum_{i=1}^{N} \frac{i}{N}\right) \\
& =\frac{\sigma^{2}}{N}(1+2 N-2-N) \\
& =\sigma^{2}\left(1-\frac{1}{N}\right)
\end{aligned}
$$

When the sample span is greater than 20 times the correlation length, the standard deviation of the mean is small and decreases with an increase in the number of sample points $\mathrm{N}$. When the correlation is zero, the process is completely independent, and the equation for the variance of the mean reduces to

$$
\sigma_{a}^{2}=\frac{\sigma^{2}}{N}
$$

which is the formula for independent samples. 
Verification of the bias of the correlogram - Figures III-31 and III-32 show the correlogram calculated from a simulated line process (with an exponential correlation of unit correlation length) using both the sample mean and population mean. The total sample span $N \cdot \Delta x$ over correlation length is 5.00 in Fig. III-31 and 1.00 in Fig. III-32. Each line process is divided into $N=100$ grid points. The plotted curves have been averaged over 100 independent line processes (100 realizations) to approximate the expected value. These figures show the systematic error caused by the substitution of the population mean $\mu$ by the sample mean $\hat{\mu}$.

The bias can be predicted from Eqs. (III-28) and (III-30). Equation (III-30) predicts that the bias is constant for different lag distance $h$, which is verified by the figures. From Eq. (III-31) we can calculate $\sigma_{\hat{\rho}}{ }^{2}=0.35$ for Fig. III-31 (total sample span/correlation length $\mathrm{N} \cdot \mathrm{a}=5.00$ or $\mathrm{a}=.05$ ) and $\sigma_{p}^{2}=0.75$ for Fig. III-32 $(\mathrm{a}=.01)$. The correlograms agree with the prediction quite we11. The exponential autocorrelation tends to zero at large lag distances, while the correlogram using the sample mean tends to a negative value which approximates $\sigma_{\rho}^{2}$.

The bias is from the true correlogram's estimate using the population mean $C_{\mu}(h)$, while $C_{\mu}(h)$ itself can deviate from the given correlogram. See the tails at large lag distance in Figs. III-3I and III-32. As we will show later, the uncertainty in estimating the correlogram is higher with a relatively small total sample span and at large lag distances. 


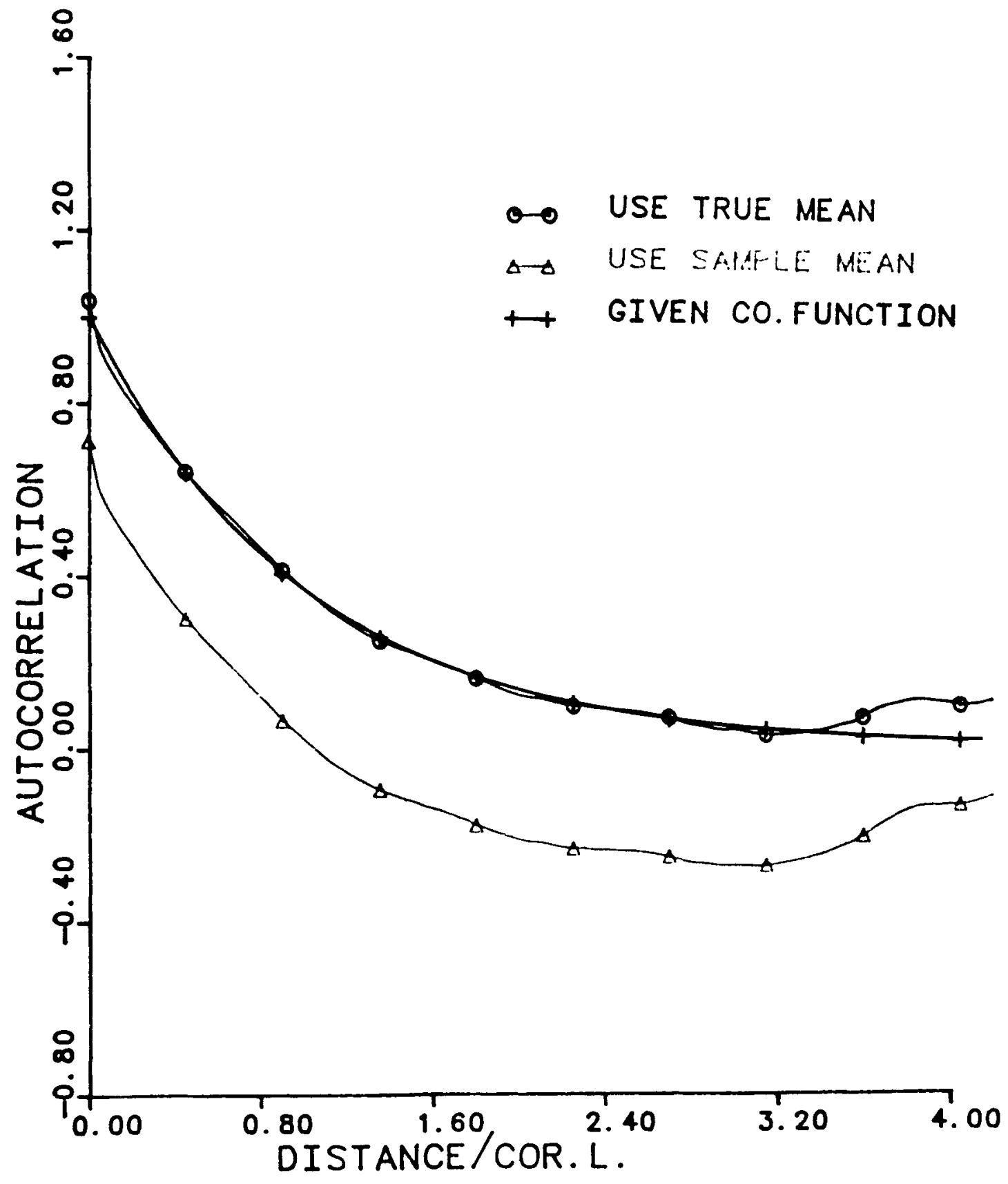

Figure III-31. Constructed autocorrelation for sample span/ correlation length $=5$. 


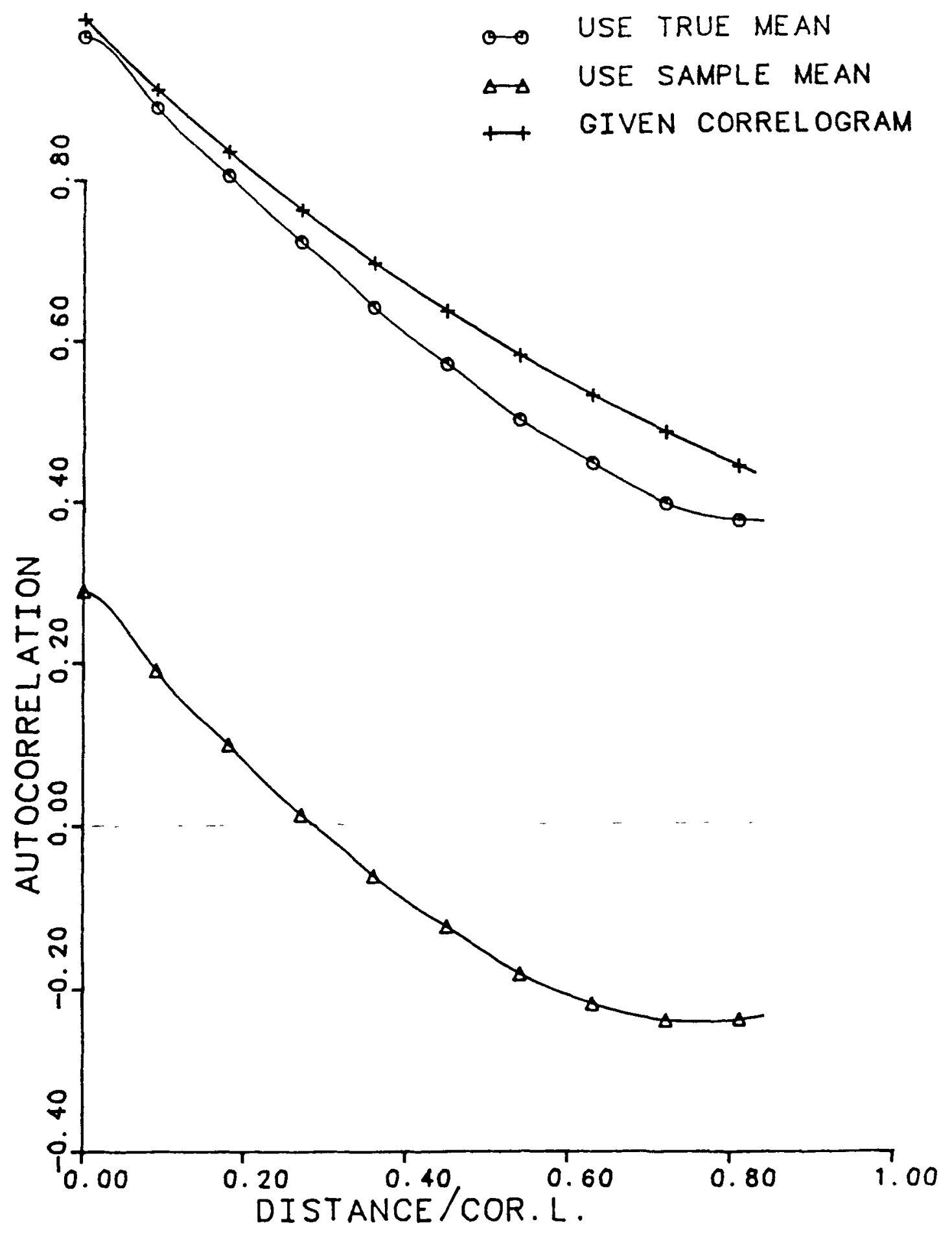

Figure III-32. Constructed autocorrelation for sample span/ correlation length $=1$, 
A similar argument holds for the variance or confidence interval of the autocorrelation calculated from sample data. Figures III-33 and III-34 show the simulated results of line processes generated in the same manner as Figs. III-31 and III-32. In Fig. III-34, the standard deviation $\sigma=\sqrt{C_{\mu}(0)}$ of line process $i$, divided into 100 points, uses population mean $\mu$. The four curves, plotted as a function of correlation length, have been calculated from 100 independent lines (100 realizations) for each correlation length. They are,

(1) the averaged standard deviation,

$$
\bar{\sigma}=\frac{1}{N} \sum_{i=1}^{N} \sigma_{i}
$$

(2) the estimated standard deviation of $\sigma=\sqrt{\mathrm{C}(0)}$,

$$
\left(\sigma \text { of } \sigma_{i}\right)^{2}=\frac{1}{N} \sum_{i=1}^{N}\left(\sigma_{i}-\bar{\sigma}\right)^{2}
$$

(3) the maximum $\sigma$,

$$
\sigma_{\max }=\max \left\{\sigma_{1}, \ldots, \sigma_{N}\right\}
$$

and

(4) the minimum $\sigma$,

$$
\sigma_{\min }=\min \left\{\sigma_{1}, \ldots, \sigma_{N}\right\}
$$




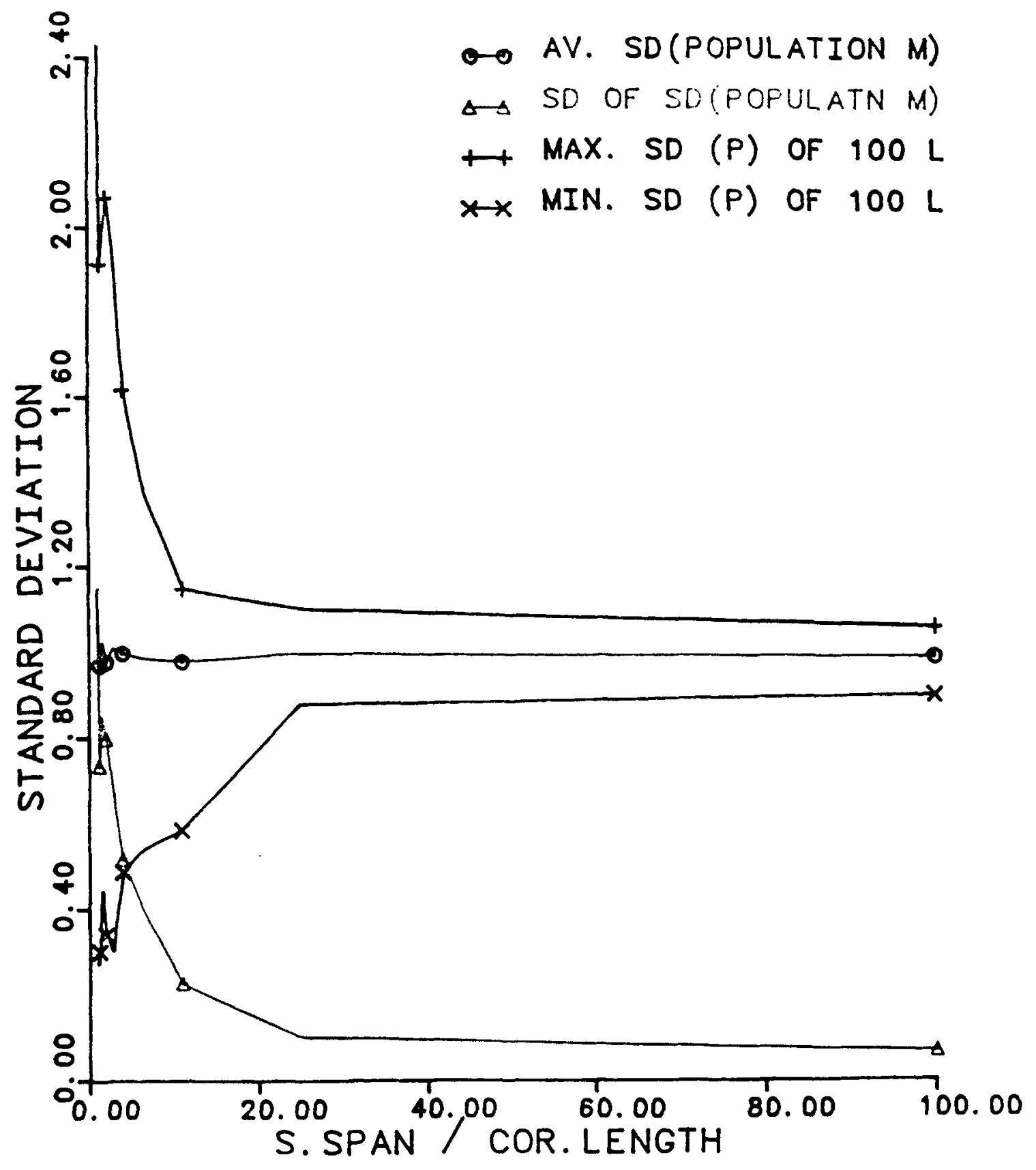

Figure III-33. The variability of line processes. 


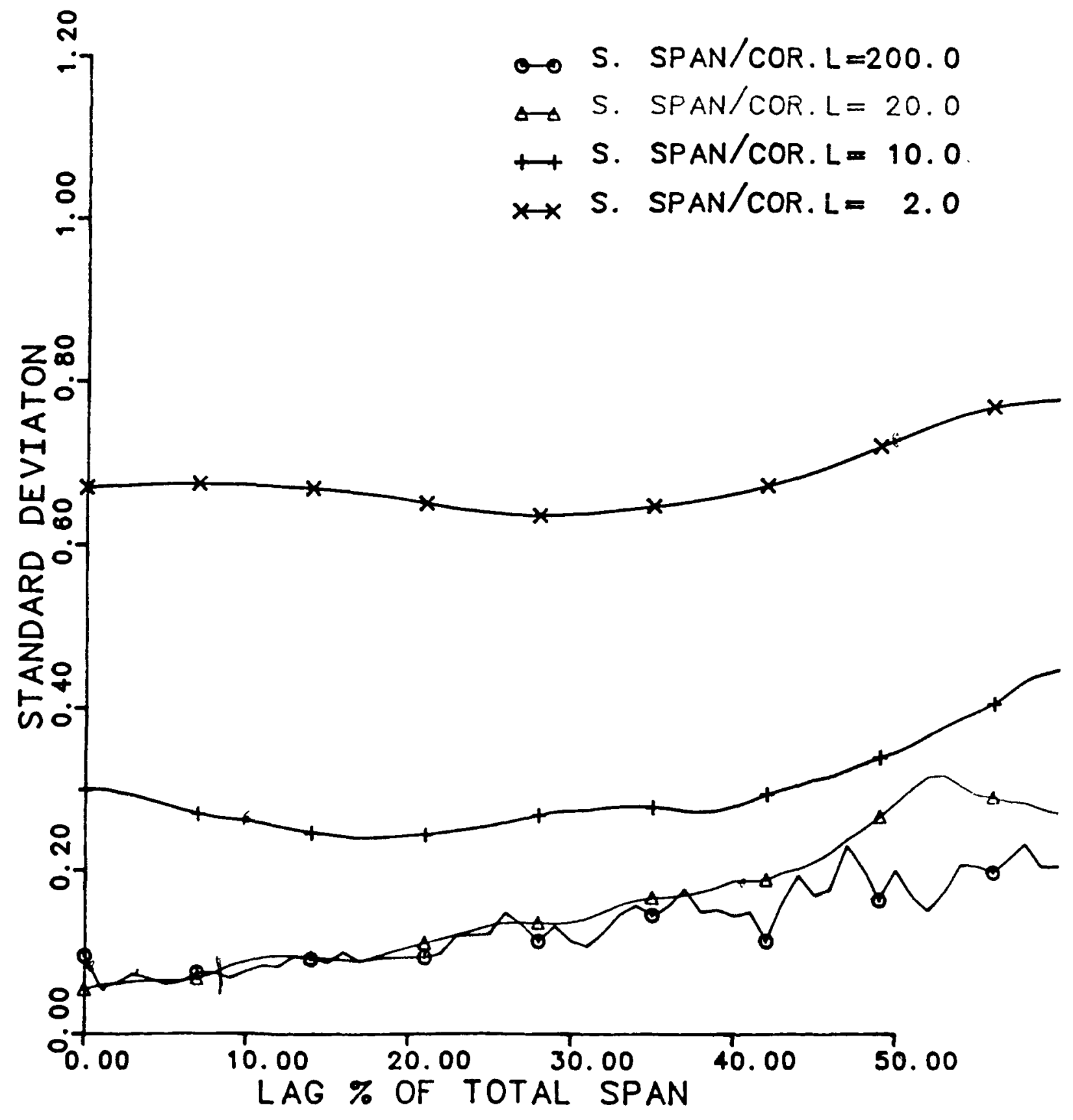

Figure III-34. The variability of the covarlogram. 
As the sample span becomes less than about 20 times the correlation length, the standard deviation of the sample variance increases rapidly and the possible outcomes of the sample standard deviation becomes erratic, even though the average standard deviation using population mean is not biased.

Figure III-34 is the standard deviation of the correlogram of s1mulated data from $0 \%$ to $60 \%$ lag distance of the total sample span

$$
\left\{\text { SD of } C_{\mu}(h)\right\}^{2}=\frac{1}{N} \sum_{i=1}^{N}\left\{C_{\mu}(h){ }_{i}-\overline{C_{\mu}(h)}\right\}^{2}
$$

Each curve is from 100 line processes of the same correlation length and each line process divided into $\mathrm{N}=100$ data points. These curves show that the uncertainty in estimating the correlogram is again controlled by the ratio of the total sample span to the correlation length, with a critical number 20 for the exponential correlation used. The uncertainty increases quickly as the ratio becomes lower than 20 , and is relatively constant for different lag distances for a fixed total sample span/correlation length. But there is a slight Increase at larger $1 \mathrm{ag}$ distances because of the decrease in the number of data points in calculating $C_{\mu}(h)$. For larger sample spans (total sample span/correlation length greater than) the effect of the correlation on the uncertainty is negligible, as can be seen from the two curves with a ratio of 20 and 200 . When the grid size is equal to or greater than the correlation length, the deviation appears less smooth, as in the curve with a ratio of 100 . 
Variogram and Its Estimate- To avoid the systematic error caused by the variance of the sample mean, a variogram, which is defined independently of the mean,

$$
2 \gamma(h)=E\left[\{z(x)-z(x+h)\}^{2}\right]
$$

can be used to regress the standard deviation and the correlation length with a given model. The varlogram (sometimes called semi-variogram, due to the presence of the 2) can be related to the correlogram:

$$
\begin{aligned}
\gamma(h) & =\frac{1}{2} E\left[\left(z_{x}-z_{x+h}\right)\right]^{2} \\
& =\frac{1}{2} E\left[\left(\left\{z_{x}-\mu\right\}-\left\{z_{x+h}-\mu\right\}\right)^{2}\right] \\
& =\frac{1}{2} E\left[\left(z_{x}-\mu\right)^{2}+\left(z_{x+h}-\mu\right)^{2}-2\left(z_{x}-\mu\right)\left(z_{x+h}-\mu\right)\right] \\
& =\frac{1}{2} E\left[\left(z_{x}-\mu\right\}^{2}+\left(z_{x+h}-\mu\right)^{2}-E\left[\left(z_{x}-\mu\right)\left(z_{x+h}-\mu\right)\right]\right. \\
& =\sigma^{2}-C(h)
\end{aligned}
$$

Where stationarity is used to assume that the expected value at $\mathrm{x}$ is equivalent to that at $\mathrm{x}+\mathrm{h}$.

In practice, let $V(h)$ represent the estimate of the variogram $\gamma(\mathrm{h})$

$$
\begin{aligned}
V(h) & =\frac{1}{2} N \sum_{i=1}^{N}\left[\left(z_{i}-z_{i+k}\right)^{2}\right] \\
& =\frac{1}{2} N \sum_{i=1}^{N}\left[\left(\left\{z_{i}-\mu\right\}-\left\{z_{i+k}-\mu\right\}\right)^{2}\right]
\end{aligned}
$$




$$
\begin{aligned}
& =\frac{1}{2 N} \sum_{i=1}^{N}\left[\left(z_{i}-\mu\right)^{2}+\left(z_{i+k}-\mu\right)^{2}-2\left(z_{i}-\mu\right)\left(z_{i+k}-\mu\right)\right] \\
& =\frac{1}{2 N} \sum_{i=1}^{N}\left[\left(z_{i}-\mu\right)^{2}+\left(z_{i+k}-\mu\right)^{2}\right] \frac{1}{N}-\sum_{i=1}^{N}\left[\left(z_{i}-\mu\right)\left(z_{i+k}-\mu\right)\right] \\
& =\sigma^{2}-C_{\mu}(h)
\end{aligned}
$$

$\sigma$ is an estimate of the population standard deviation using the population mean $\mu$ which does not appear explicitly in the equation for the variogram. On the other hand, if the sample mean $\hat{\mu}$ is assumed Implicitly, the result will be

$$
\begin{aligned}
& \left.V(h)=\frac{1}{2 N} \sum_{i=1}^{N}\left[z_{i}-z_{i+k}\right)^{2}\right] \\
& =\frac{1}{2 N} \sum_{i=1}^{N}\left[\left(\left\{z_{i}-\hat{\mu}\right\}-\left\{z_{i+k}-\hat{\mu}\right\}\right)^{2}\right] \\
& \frac{1}{2 N} \sum_{i=1}^{N}\left[\left(z_{i}-\hat{\mu}\right)^{2}+\left(z_{i+k}-\hat{\mu}\right)^{2}-2\left(z_{i}-\hat{\mu}\right)\left(z_{i+k}-\hat{\mu}\right)\right] \\
& =\frac{1}{2 N} \sum_{i=1}^{N}\left[\left(z_{i}-\hat{\mu}\right)^{2}+\left(z_{i+k}-\hat{\mu}\right)^{2}\right]-\frac{1}{N} \sum_{i=1}^{N}\left[\left(z_{i} \hat{-} \mu\right)\left(z_{i+k}-\mu\right)\right] \\
& =\hat{\sigma}^{2}-C_{\hat{p}}(h)
\end{aligned}
$$

where $\hat{\sigma}$ is an estimate of the standard deviation using the sample mean. This shows that the bias caused by the deviation of the sample mean from the population mean is the same in $\hat{\sigma}=c_{\hat{p}}(0)$ and in $c_{\hat{p}}(h)$ 
and, therefore will cancel out.

From this relationship, a correlation function model can also be used for the variogram without the bias caused by the deviation in the sample mean. However, the uncertainty of the variogram is st111 large if the total sample span is not at least ten times larger than the correlation length.

Figure III-35 shows the standard deviation of the variogram of the same simulated data as for Fig. III-34 from $0 \%$ to $60 \%$ lag distance of the total sample span. The effect of the total sample span/correlation length also shows a critical number of 20 as for the correlogram. Below this ratio the uncertainty of the variogram increaes. The curve with ratio 200 does not follow a consistent trend, except for the zero-lag point, since the grid size is smaller than the correlation length and the data can be considered virtually uncorrelated.

The observation is that for a total sample span/correlation length greater than 20 , the uncertainty is about the same for both the variogram and the correlogram.

What is different from the correlogram case is that the uncertainty increases with lag distance, particularly for curves with smaller total sample span relative to the correlation length. One reason is that the variogram has a smaller value when the lag distance is less than the correlation length. Also the correlation makes this portion of the variogram more regular. By definition the variogram has a zero value (and therefore zero standard deviation) with lag distance equals zero, which is not the case for th correlogram (the correlogram can be normalized by $\mathrm{C}(0)$ to force the zero-lag point 


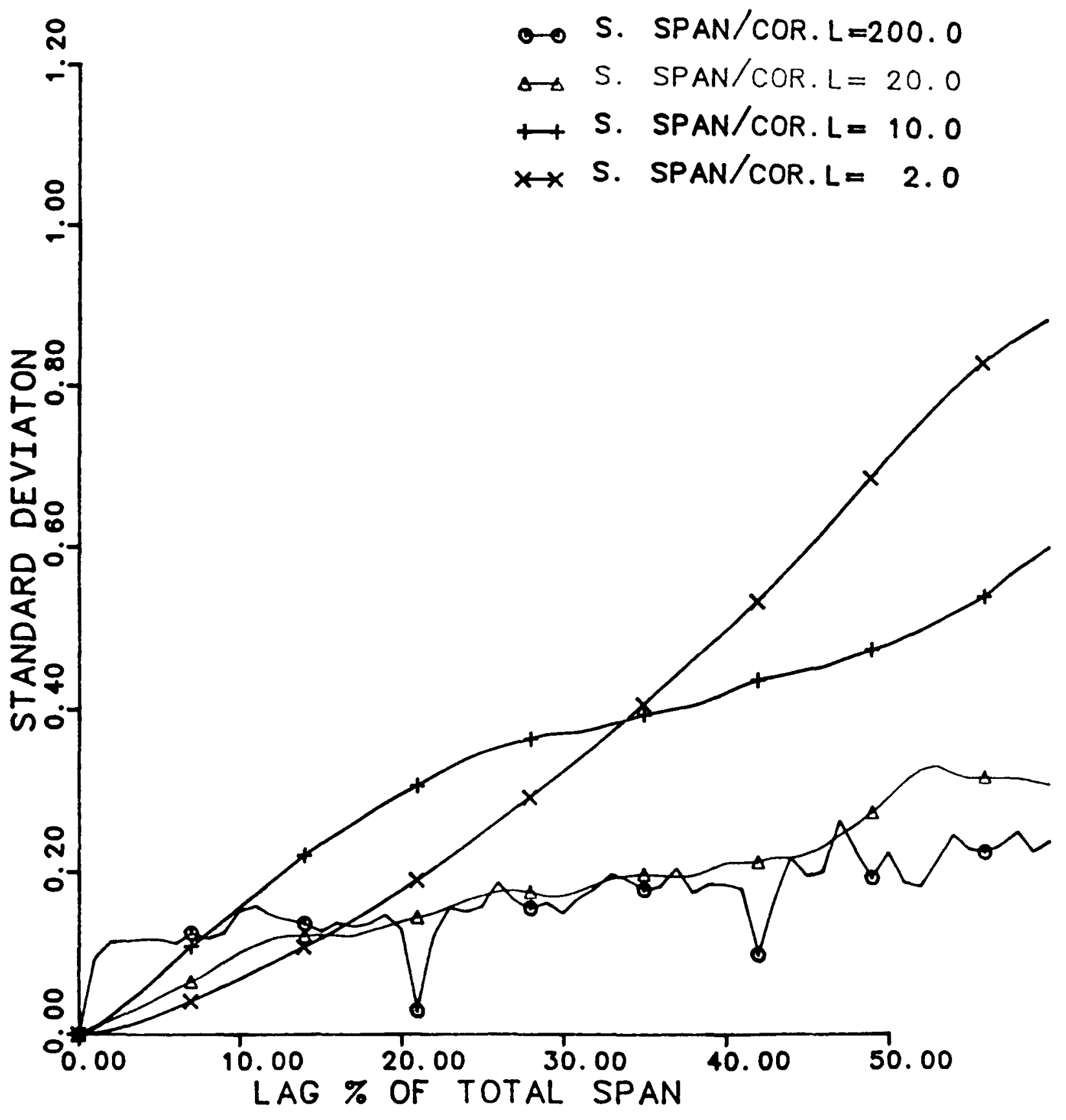

Figure III-35. Uncertainty of the variogram. 
equal to unity but the uncertainty in the estimate of $c(0)$ remains). The conclusion is that the correlated portion of the variogram always shows a smaller uncertainty than the correlogram, i.e.

$$
\sigma_{\operatorname{var}}<\sigma_{\text {corr }} \text { when } \frac{h}{\lambda}<1
$$

Figure III-36 compares the two smaller sample span curves from Figs. III-34 and III-35. For the curves with total sample span/correlation length 2, a lag distances less than $50 \%$ of the total span will have $\mathrm{h}<\lambda$ and the standard deviation is smaller for the vriogram. For the other pai with ratio $10,10 \%$ lag of the total span will have $h<\lambda$ and also the uncertainty for the variogram is smaller.

The spherical model is commonly used for variograms. Curve-fitting an exponential model with the spherical model turns out an equivalent correlation length about twice that for the exponential mode1. Subsequently the equivalent correlation length was used to generate and plot curves using the spherical model similarly to those In Figs. III-33 and III-34, and the results were about the same. This shows that the conclusions drawn with the exponential model can be similarly applied to other correlation models if an equivalent correlation length can be established.

Standard for Correlation - Recommendations for estimating spatial correlation are,

1) Very small correlation - When the correlation length is smaller than the grid size 


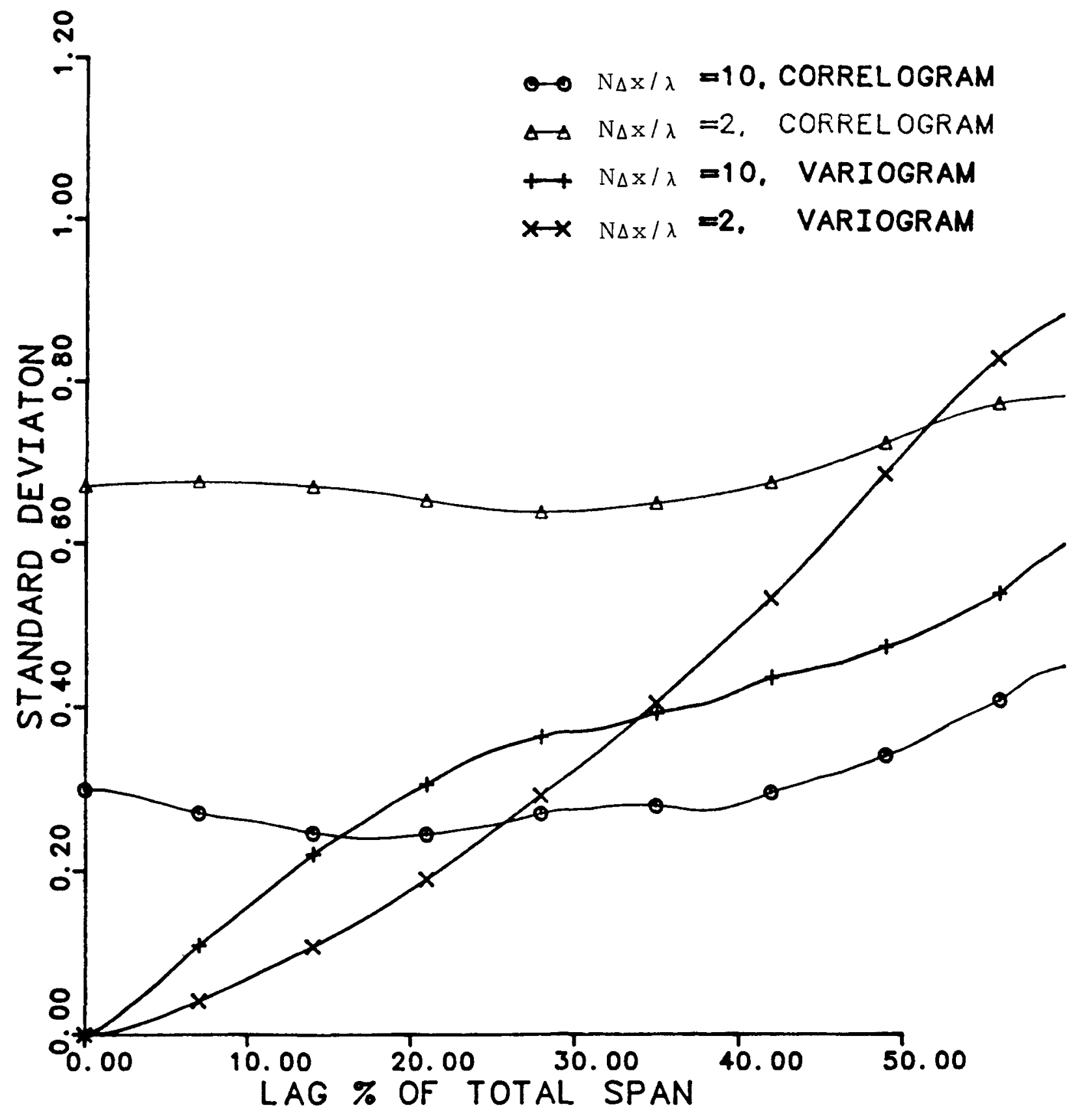

Figure III-36. A comparison between the correlogram and the variogram. 
the data will appear independent and there is no way to estimate the actual correlation from the generated data. Only the standard deviation will be significant in this case.

2) Small correlation - When the total sample span is larger than 20 times the correlation length (10 times for the spherical model),

$$
\mathrm{N} \cdot \Delta \mathrm{x}>\lambda \cdot 20
$$

the uncertainty is small in estimating either the variogram or the correlogram, and the bias in estimating the correlogram using sample mean is also sma11. As far as accuracy is concerned, there is little difference in using either.

3) Large correlation - When the total sample span is less than 20 times the correlation length (10 times for the spherical model),

$$
\mathrm{N} \cdot \Delta \mathrm{x}<\lambda \cdot 20
$$

the estimate of correlogram using the sample mean is substantially biased and should not be used. The uncertainty is large in estimating either the variogram or the correlogram, but it is smaller within the correlated range for the variogram. Also the correlogram cannot be unbiasedly estimated without knowing the population mean. Therefore, the variogram should be used.

Input/output parameters - The parameters curve-fitted to the variogram calculated from a simulated process (output parameters) are more or less different from those given to the generator (input parameters). 
Which should be used as the standard? In order to use the data in a reservoir simulation study, we would expect the data to have the same correlation structure as specified. Therefore the output parameters should be used as the standard.

Fixing the set of random numbers used in the simulation, the input standard deviation and correlation length can be changed to achieve the specified output parameters. The generator can automatically least-square-fit a variogram to the variogram calculated from generated data to get estimates of the output standard deviation and correlation length. For example, when a standard deviation of 100 is required but the first run of a simulation generates a varogram best fitted with a standard deviation equal to 94 , we can input a standard deviation of 105 for the next run that would probably produce 100 in the output standard deviation.

Non-Normal Distribution - As to the distribution of the points, probability plots show that line process generated by the TBM is approximately normally distributed. Figure III-37, a probability plot of normalized data value (permeability) versus the inverse error function (Jensen et al. 1986), shows different line processes covering different total sample spans over correlation length. For large sample spans over correlation length, the data are very close to the theoretical normal. The deviation from the normal distribution is largely caused by the correlation between sample points. This indicates that for small sample span or highly correlated data it is not easy to test the normality of the distribution. 


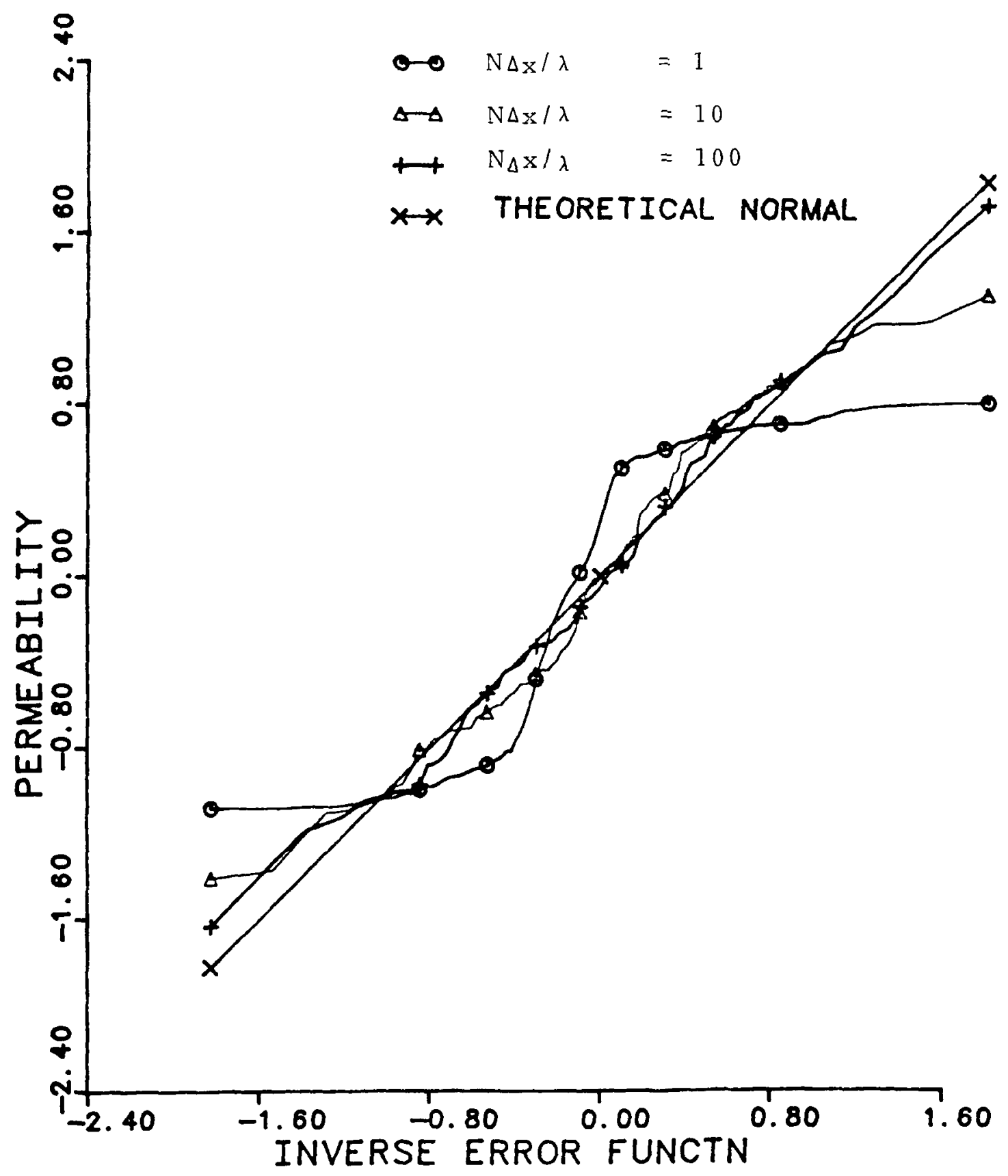

Figure III-37. Effect of correlation on the cumulative density function 
If a non-normal distribution is to be simulated, a transformation can be done after the normally distributed process has been generated, in a reverse direction to the transformation to normal. For example, an exponential of the process which is normally distributed would result in a log-normal distribution, since the logarithm of this will bring it back to a normal distribution. The relation between normal data $z$ from the TBM and log-normal data can be derived:

$$
Z_{1 \circ \text {-normal }}=\exp (z)
$$

while for $p$-normal where $p \neq 0$

$$
z_{p-\operatorname{norma1}}=[\max (0, z \cdot p+1)]^{1 / p}
$$

In this tranformation any negative number will be set to zero, since we cannot raise a negative number to real power.

After the transformation to p-normal, the coefficient of variation (the ratio of the standard deviation to the mean) will be changed. Because the transformation involves raising the variable to the power $\mathrm{p}$, addition or subtraction would change the index $\mathrm{p}$. Therefore only multiplication can be used after the transformation because this will not change the coefficient of variation.

To achieve the required coefficient of variation after the transformation, a different coefficient of variation should be used to generate the data. Because the standard deviation is a parameter to be adjusted in the generating program, it is more convenient to change the input standard deviation also to fit the required coefficient of variation after the transformation to p-normal, i.e. the output parameters will be estimated after both the transformation 
to p-normal and the adjustment for the mean. The adjustment on the mean will be achieved by multiplication

$$
k_{i}=\frac{z_{i} \cdot \mu}{\frac{1}{N} \sum_{i=1}^{N} z_{i}}
$$

so that

$$
\frac{1}{N} \sum_{i=1}^{N} k_{i}=\frac{1}{N} \sum_{i=1}^{N} \frac{z_{i} \cdot \mu}{\frac{1}{N} \sum_{j=1}^{N} z_{j}}=\mu
$$

Alternatively the p-normal average can be used instead of the arithmetic average, but it is too complicated and usually not readily available.

After the transformation to p-normal, the variogram may be changed a little, but the shape is about the same, and the correlation length is little affected. Figure II-38 shows the effect of this transformation on the variogram with a unit coefficient of variation before transformation and normalized to the same variance after the transformation. $p=1$ means normally distributed, and the option of omitting the negative values is included since that is required in transforming to p-normal.

With a coefficient of variation as large as 1 , a substantial part of the data would be negative for a true normal distribution. In a permeability field, or any p-normal distribution where $p \neq 1$, there can not exist negative values. Setting the negative values to zero solves this problem but at the same time distorts the probability 


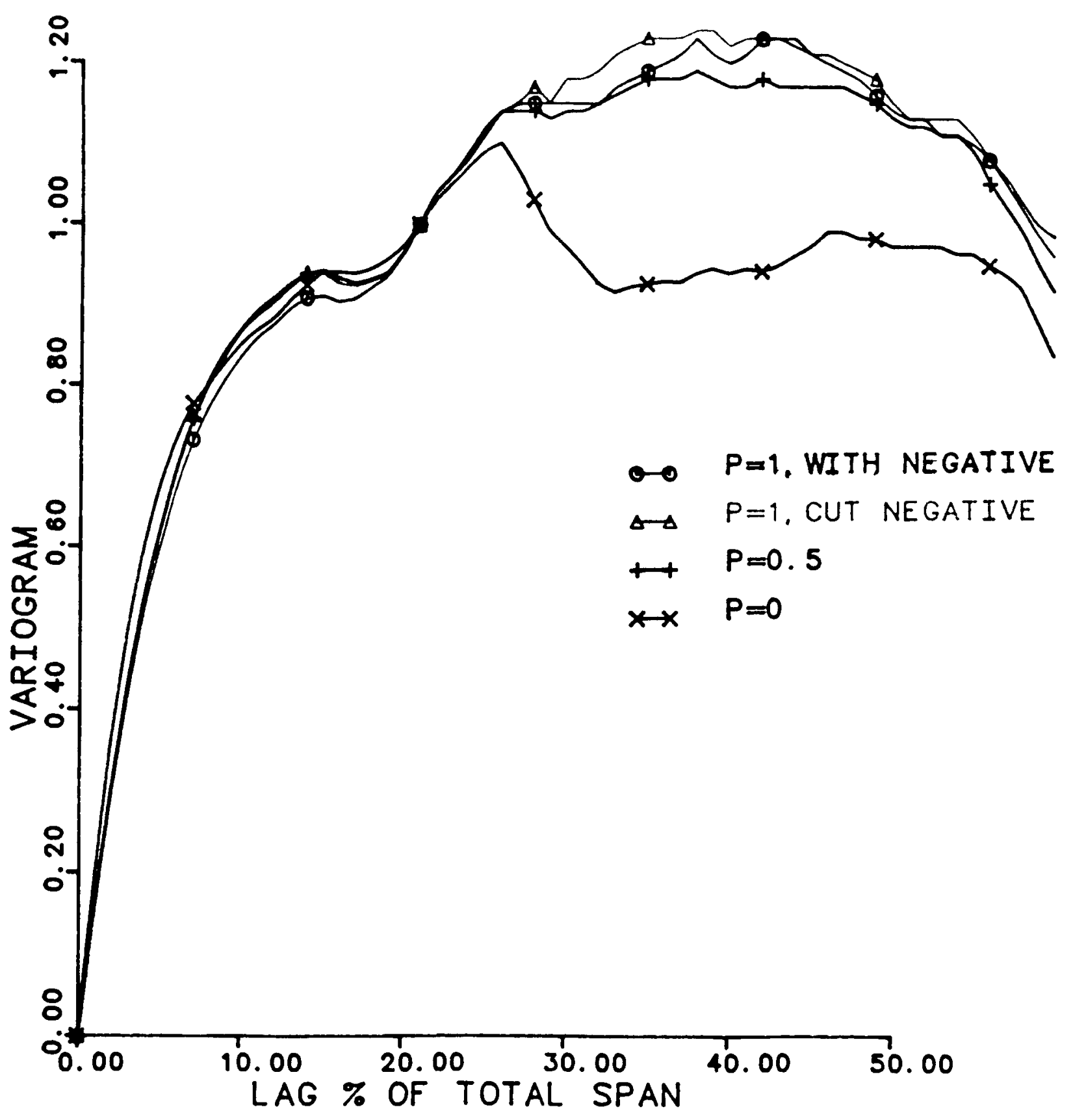

Figure III-38. Effects of p-normal transformation of variogram. 
distribution. Figure II-39 shows how the transformation affects the probability density function.

Why does the transformation change the probability density much more than the variogram? It is because the probability density is grouped by the value of the data which is what the transformation changes directly, but the variogram shows the variation over a separation distance and is averaged over the whole area; therefore, the change in data value at one point would not be much different from that at the other and the variation between these two points would remain much the same. For the portion of negative values set to zero, it will appear collectively in the probability density function at one end, but in the variogram it is scattered among the averaged variations and therefore will not affect the shape (the standard deviation will be different).

For transformation to approximately normal, see Jensen's paper (1985)

Spectrum - The spectra density function w can be calculated from the generated data by

$$
w(\alpha)=\frac{1}{S^{2} N}\left\{\left(\sum z_{i} \cos \alpha x_{i}\right)^{2}+\left(\sum z_{i} \sin \alpha x_{i}\right)^{2}\right\}
$$

Theoretically the spectral density contains as much information as the autocorrelation, and a relation between the two is unique. It also contains the standard deviation explicitly and therefore is subject to the same error as the correlogram. Furthermore, the spectrum for a 


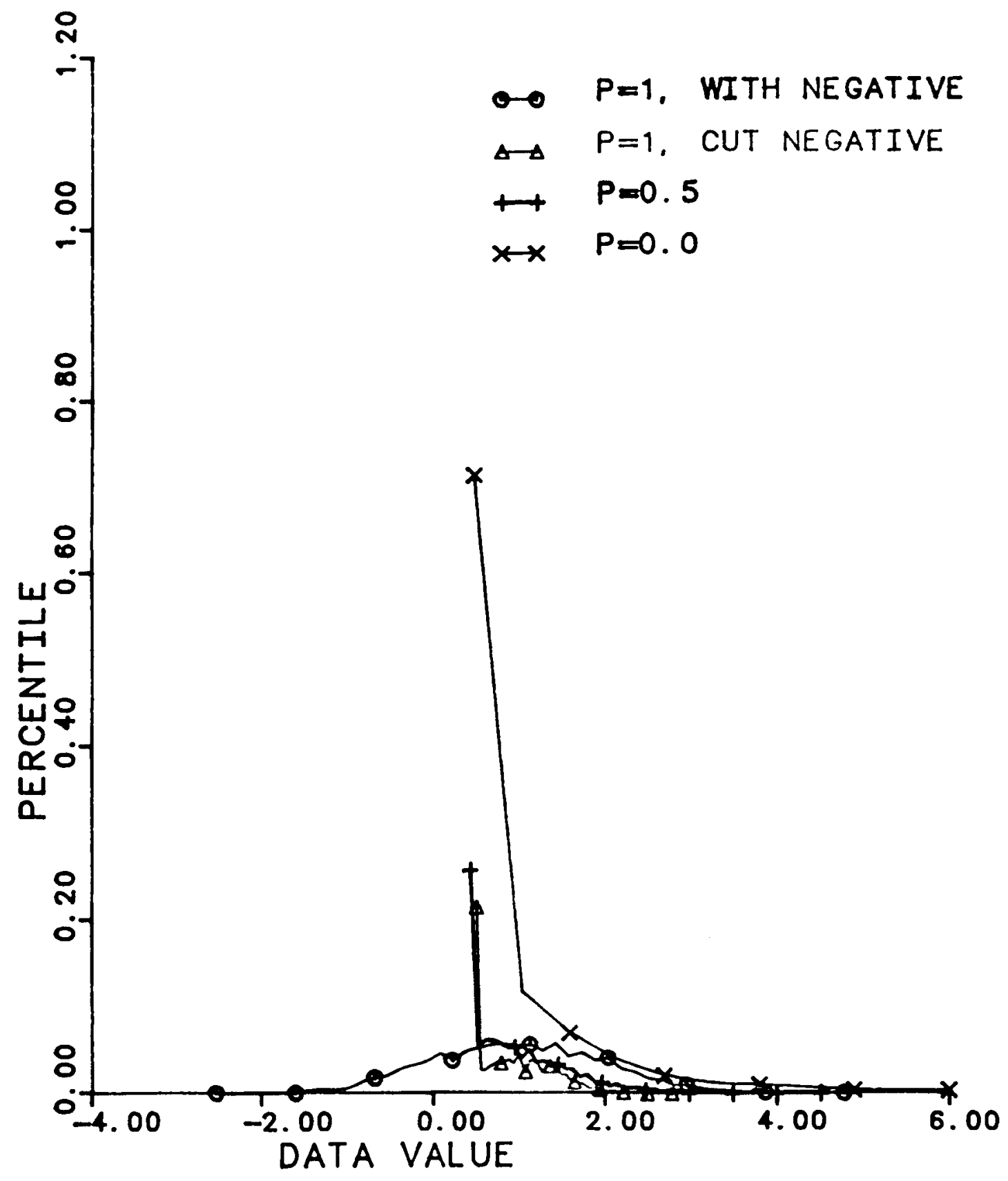

Figure III-39. Effects of transform of PDF. 
single realization is quite irregular and cannot be used quantitatively to estimate the spectral density.

Qualitatively, the spectrum can be used to check the correspondence of the simulated data with the model used by plotting the theoretical together with the spectrum calculated from the generated data. 


\section{APPENDIX A - BIAS AND ERROR OF QUANTILE ESTIMATORS}

We wish to eatablish the bias and standard error associated with quantities of the form

$$
\hat{V}=\left(\hat{Y}_{r}-\hat{Y}_{t}\right) / \hat{Y}_{s}
$$

where $\hat{\mathrm{Y}}_{\mathrm{u}}$ is an estimate of the quantile of the log-normal distribution for the decimal fraction $u$ and $0 \leqq t \leqq s \leqq r \leqq 1$. The theory for this problem is set out in Chapter 10 of Kendall and Stuart (1977). We consider the general problem initially and then take the specific case of the Dykstra-Parsons coefficient, $V_{D P}$, for which $r=s=0.50$ and $t$ $=0.1587$.

For a sample of size $N$ of the random variable $y$ with p.d.f. $P_{y}$,

$$
\hat{Y}_{u} \text { is } N\left[Y_{u}, y(1-u) /\left(N P_{y}{ }^{2}\left(Y_{u}\right)\right)\right]
$$

when $\mathrm{N}$ is large. Suppose that $\ln (\mathrm{y})$ is $\mathrm{N}\left(\mathrm{m}_{\mathrm{x}}, \mathrm{s}_{\mathrm{x}}{ }^{2}\right)$. It follows from the properties of the log-normal distribution (Johnson and Kotz, 1970) and Eq. (A-2) that

$$
E\left(\hat{Y}_{u}\right)=\exp \left(m_{x}+s_{x} \cdot w_{u}\right)
$$

and

$$
\operatorname{Var}\left(\hat{Y}_{u}\right)=A u(1-u) \exp \left(2 m_{x}+2 s_{x} \cdot w_{u}+w_{u}^{2}\right)
$$

where $A=2 \pi s{ }_{x}^{2} N^{-1}, w_{u}=\phi^{-1}(u), E(\cdot)$ denotes the expectation, $\operatorname{Var}(\cdot)$ denotes the variance, and $\phi$ is the probability integral (Johnson and 
Kotz, 1970). Kendall and Stuart (1977) also show that, for two quantile estimates $\hat{\mathrm{Y}}_{\mathrm{u}}$ and $\hat{\mathrm{Y}}_{\mathrm{v}}$,

$$
\operatorname{Cov}\left(\hat{Y}_{u}, \hat{Y}_{v}\right)=\operatorname{Au}(1-v) \exp \left[2 m_{x}+s_{x}\left(w_{u}+w_{v}\right)+1 / 2\left(w_{u}^{2}+w_{v}^{2}\right)\right]
$$

where $\operatorname{Cov}(\cdot, \cdot)$ denotes the covariance.

Equation $(A-1)$ is a nonlinear combination of the quantile estimates. Some work is involved in establishing $E(\hat{V})$ and $\operatorname{Var}(\hat{V})$ in terms of the quantile estimate properties. Consider a function $g$ of the form

$$
g\left(x_{1}, x_{2}, x_{3}\right)=\left(x_{3}-x_{1}\right) / x_{2}
$$

By taking a Taylor series expansion of $g$ about the expected values of random variables $x_{1}, x_{2}$, and $x_{3}$, and truncating we obtain for the expectation and variance of $g(x)$ :

$$
\begin{aligned}
E[g(x)]= & g(a)+1 / 2\left[\sum_{g_{i i}} \operatorname{Var}\left(x_{i}\right)+\right. \\
& \left.\sum \sum g_{i j} \operatorname{Cov}\left(x_{i}, x_{j}\right)\right] \\
& i \neq j
\end{aligned}
$$

and

$$
\begin{aligned}
\operatorname{Var}[g(x)]= & \sum\left(g_{i}\right)^{2} \operatorname{Var}\left(x_{i}\right)+ \\
& \sum \sum g_{i} g_{j} \operatorname{Cov}\left(x_{i}, x_{j}\right) \\
& i \neq j
\end{aligned}
$$


where $x=\left(x_{i}, x_{2}, x_{3}\right), a=\left(E\left(x_{1}\right), E\left(x_{2}\right), E\left(x_{3}\right)\right), g_{i}=\left(\partial g / \partial x_{i}\right)$ evaluated at $x=a, g_{i j}=\left(\partial^{2} g / \partial x_{i} \partial x_{j}\right)$ at $x=a$, and the summations are $i, j=1$ to 3 . For the case under consideration, Eqs. (A-7) and (A-8) are correct to order $\mathrm{N}^{-1}$.

Application of Eqs. A-3 through A-5 in A-7 yields

$$
\begin{aligned}
E(\hat{V})= & V+\operatorname{Aexp}\left(-s_{x} w_{s}\right)\left\{V s(1-s) f\left(w_{s}\right)+\right. \\
& t(1-s) \exp \left[s_{x} w_{t}+1 / 2\left(w_{t}^{2}+w_{s}^{2}\right)\right]- \\
& \left.s(1-r) \exp \left[s_{x} w_{r}+1 / 2\left(w_{s}^{2}+w_{r}^{2}\right)\right]\right\}
\end{aligned}
$$

where $f\left(w_{u}\right)=\exp \left(2 s_{x} w_{u}+w_{u}{ }^{2}\right)$. The bias $m_{v}$ is obtained from $m_{v}=$ $\mathrm{E}(\hat{\mathrm{V}})-\mathrm{V}$. Hence, the bias is given by the second and subsequent terms on the right side of Eq. (A-9). Equations (A-3) through (A-5) in (A-8) yield

$$
\begin{aligned}
\operatorname{Var}(\hat{V})= & \operatorname{Aexp}\left(-2 s_{x_{s}} w_{s}\right)\left\{t(1-t) f\left(w_{t}\right)+\right. \\
& \left.r(1-r) f\left(w_{r}\right)-2 t(1-r) h\left(w_{t}, w_{r}\right)\right]+ \\
& V^{2} s(1-s) f\left(w_{s}\right)+2 V t(1-s) h\left(w_{t}, w_{s}\right)- \\
& \left.2 \operatorname{Vs}(1-r) h\left(w_{s}, w_{r}\right)\right\}
\end{aligned}
$$

where

$$
h\left(w_{u}, w_{v}\right)=\exp \left[s_{x}\left(w_{u}+w_{v}\right)+1 / 2\left(w_{u}^{2}+w_{v}^{2}\right)\right]
$$

For the special case of the $V_{D P}$ estimator, $r=s=0.50$ and $t=$ 0.1587 . Hence $w_{t}=-1$ and $w_{S}=w_{r}=0.0$. Equations (A-9) and $(A-10)$ become 


$$
E\left(\hat{V}_{D P}\right)=V_{D P}-0.7488 s_{x}^{2} \exp \left(-s_{x}\right) N^{-1}
$$

and

$$
\operatorname{Var}\left(\hat{\mathrm{V}}_{\mathrm{DP}}\right)=2.207 \mathrm{~s}_{\mathrm{x}}{ }^{2} \exp \left(-\mathrm{s}_{\mathrm{x}}\right) \mathrm{N}^{-1}
$$

Hence, the bais $m_{v}$ and standard error $s_{v}$ are

$$
m_{\mathrm{v}}=-0.7488 \mathrm{~s}_{\mathrm{x}}^{2} \exp \left(-\mathrm{s}_{\mathrm{x}}\right) \mathrm{n}^{-1}
$$

and

$$
s_{v}=1.486 s_{x} \cdot \exp \left(-s_{x}\right) N^{-1 / 2}
$$

For the Dykstra-Parsons coefficient, $V_{D P}=1-\exp \left(-s_{x}\right)$. Hence, Eqs. $(A-13)$ and $(A-14)$ may be expressed in terms of $V_{D P}$. 


\section{APPENDIX B - BIAS AND ERROR OF THE LORENZ ESTIMATOR}

This section describes the Monte Carlo simulations which were made to establish the bias and standard error behavior of the Lorenz estimator. A log-normal p.d.f. is assumed for the permeability data. The algorithm steps are as follows:

1. Obtain $\mathrm{N}$ normally distributed data, $\mathrm{x}_{1}, \mathrm{x}_{2}, \mathrm{x}_{3}, \ldots, \mathrm{x}_{\mathrm{N}}$ where $\mathrm{x}$ is $N(0,1)$. The commercial IMSL routine, called GGNQF, was used.

2. Transform the data to a log-normal distributed set.

$$
y_{i}=\exp \left(a \cdot x_{i}\right),
$$

for $i=1,2, \ldots, N$, and where $a=2 \operatorname{erf}^{-1}\left(L_{C}\right)$ and $L_{C}$ defines the Lorenz coefficient of the data. We started at $\mathrm{L}_{\mathrm{C}}=0.3$ and incremented in steps of 0.1 to $\mathrm{L}_{\mathrm{C}}=0.9$.

3. Reorder the data so that $Y_{1} \leqq Y_{2} \leqq Y_{3} \leqq \ldots \leqq Y_{n}$.

4. Estimate $\mathrm{L}_{\mathrm{C}}$ by the trapezoidal rule

$$
\hat{\mathrm{L}}_{\mathrm{C}^{\prime}}=2 / \mathrm{N} \cdot\left(\sum \mathbf{i} \cdot \mathrm{y}_{i} / \sum \mathrm{y}_{i}\right)-1 / \mathrm{N}-1
$$

where the summations are over $i$ from 1 to $N$, or by Simpson's rule

$$
\hat{\mathrm{L}}_{\mathrm{C}^{\prime \prime}}=1.0-\frac{2}{3 N}\left(1+2 \sum_{i=1}^{N-1} 2^{q(i)} \sum_{j=1}^{i} y_{j=1} / \sum y_{i}\right)
$$

where $q(i)$ is the modulo two function (i.e. $q(i)=0$ for $i$ even and $q(i)=1$ for $i$ odd).

5. Repeat steps 1 through 4 another $\mathrm{m}-1$ times to obtain $m$ estimates $\hat{\mathrm{L}}_{\mathrm{Ci}}$, or $\hat{\mathrm{L}}_{\mathrm{C} i^{\prime \prime}}, \mathrm{i}=1,2, \ldots, \mathrm{m}$. 
6. Estimate the bias, $\mathrm{m}_{\mathrm{L}}$, and sampling error, $\mathrm{s}_{\mathrm{L}}$ :

$$
\begin{aligned}
& \hat{\mathrm{m}}_{\mathrm{L}}=1 / \mathrm{m} \sum \mathrm{L}_{\mathrm{CI}}-\mathrm{L}_{\mathrm{C}}, \\
& \hat{\mathrm{s}}_{\mathrm{L}}^{2}=1 /(\mathrm{m}-1) \sum\left(\mathrm{L}_{\mathrm{CI}}-\mathrm{L}_{\mathrm{C}}-\hat{\mathrm{m}}_{\mathrm{L}}\right)^{2},
\end{aligned}
$$

where primed or double primed quantities (i.e. trapezoidal or Simpson's rule integration) are used as appropriate.

Figure III-17 is a plot of $\mathrm{N}$ versus $\hat{\mathrm{m}}_{\mathrm{L}} / \mathrm{L}_{\mathrm{C}}$ for $\mathrm{m}=2000$. Figure III-18 is a plot of $L_{C}$ versus $N$ for four different values of $\hat{s}_{L}$ ': $0.10,0.05,0.02$ and 0.01 . Because of the computational effort required to order several thousand points for small $\hat{s}_{L}$ ', m was not held constant for all simulation runs. For $\hat{s}_{L}^{\prime} \geqq 0.05, \mathrm{~m}=$ 2000; for $\hat{s}_{L}^{\prime}=0.02, m=1000$; and for $\hat{s}_{L}^{\prime}=0.01, m=400$. 


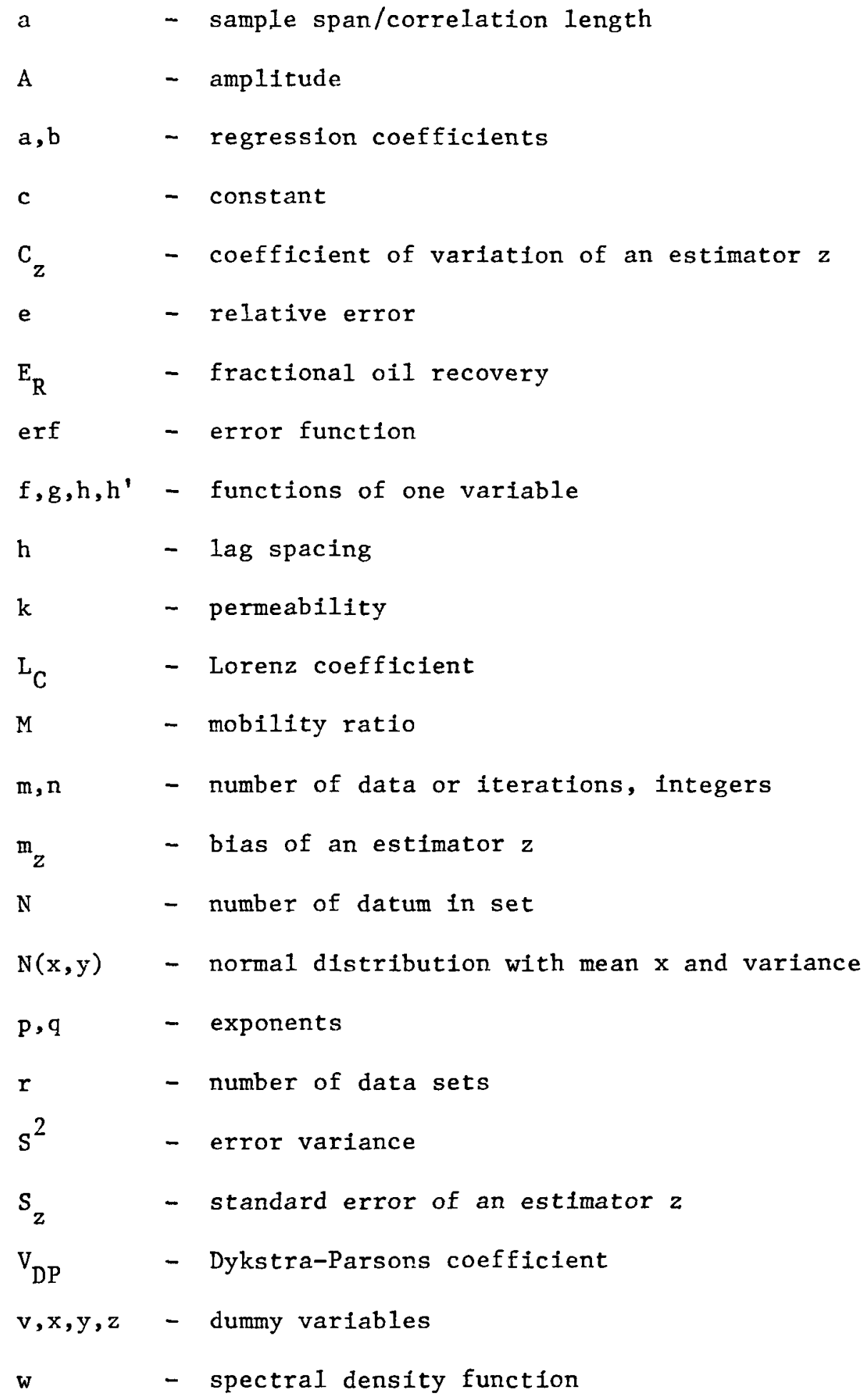

- spectral density function 


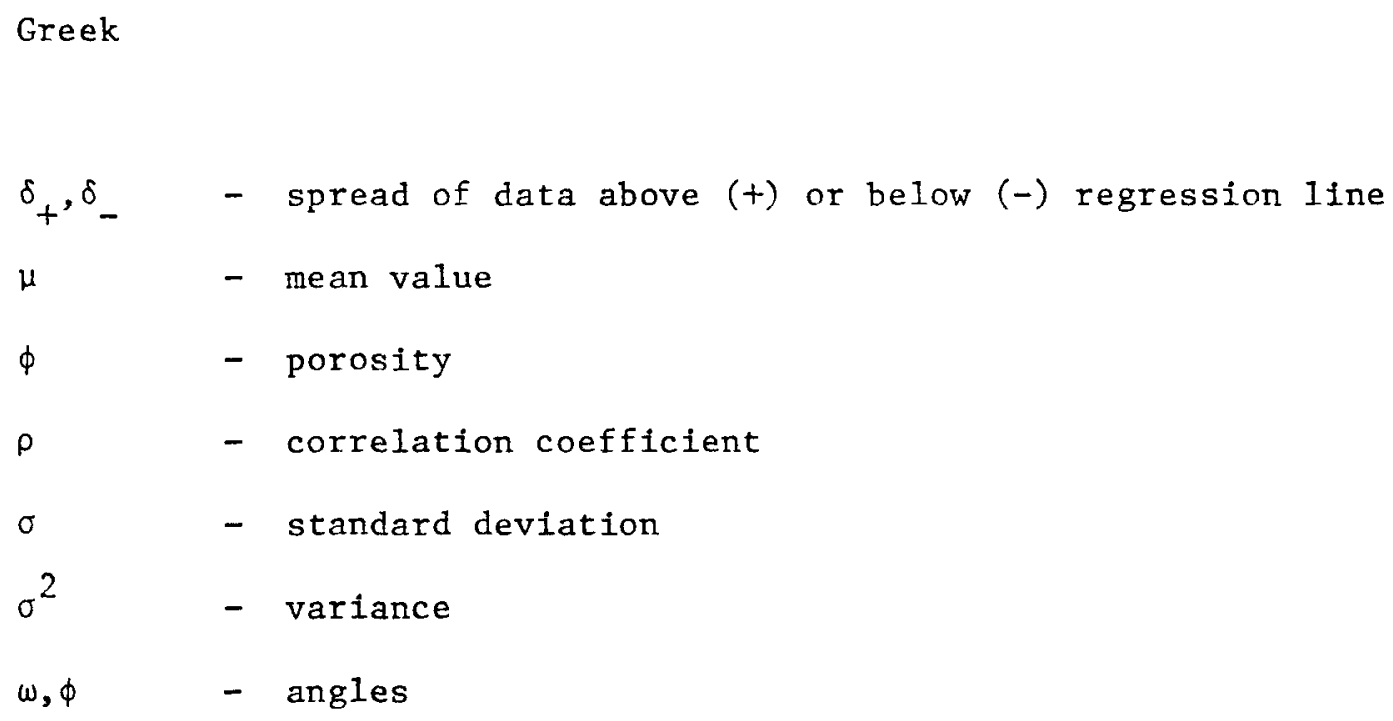
$A, B$ - pertaining to curve $A$ or $B$
i - ith datum, an integer
$\mathrm{x}, \mathrm{y} \quad$ - pertaining to variable $\mathrm{x}$ or $\mathrm{y}$ 
PART III REFERNECFS

Abramowitz, M. and Stegun, I., eds., Handbook of Mathematical Fuinctions, Dover Publications, Inc., New York (1972).

Arya, A., Hewett, T. A., Larson, R. G., and Lake, L. W., "Dispersion and Reservoir Heterogeneity," SPE 14364, presented at the 1985 Society of Petroleum Engineers Annual Technical Conference and Exhibition, Las Vegas, Nevada (Sept. 22-25).

Boyer, R. C., "Geologic Description of East Velma West Block Sims Sand Unit for an Enhanced Oil Recovery Project," paper SPE 12687 presented at the 1984 SPE/DOF Fourth Symposium on Enhanced Oil Recovery, Tulsa, April 15-18, 1984.

Brown, A. and Husseini, S., "Permeability from Well Logs, Shaybah Field, Saudi Arabia," Paper Q presented at the SPWLA Eighteenth Annual Logging Symposium, Houston, June 5-8, 1977.

Craig, F. F., Jr., The Reservoir Engineering Aspects of Waterflooding, Monograph Series, Society of Petroleum Engineers, Dallas (1971).

Emerson, J. and Stoto, M., "Exploratory Methods for Choosing Power Transformations," J. Am. Stat. Assoc. (March 1982) 77, No. 377, 103-108.

Dykstra, H. and Parsons, R. L, "The Prediction of Oil Recovery by Water Flood," API Secondary Recovery of Dil in the United States (1950) 160.

Gail, M. H. and Gastwirth, J. L, "A Scale-Free Goodness-of-Fit Test for the Exponential Distribution Based on the Lorenz Curve, J.Am Stat. Assoc. (Dec. 1978) 787.

Gastwirth, J. L., "The Estimation of the Lorenz Curve and Gini Index," Rev. Econ. and Stat. (1972) 306.

Goggin, D. J., Chandler, M. A., Kocurek, G., and Lake, L. W., "Patterns of Permeability in Eolian Deposits," SPE 14893, presented at the 1986 Society of Petroleum Engineers/Department of Energy 5th Symposium on Enhanced $0: 1$ Recover, Tulsa (April 20-23).

Gnanadesikan, R., Methods for Statistical Data Analysis of Multivariate Observations, John Wiley and Sons, New York (1977).

Hald, A., Statistical Theory with Engineering Applications, John Wiley and Sons, New York (1952).

Hoaglin, D., Mosteller, F., and Tukey, J., eds, Understanding Robust and Exploratory Data Analysis, John Viley and Sons, New York (1983), Chapters 5 and 8. 
Hoyle, M. H., "Transformations - An Introduction and a Bibliography," Int. Stat. Rev. (1973) 41, No. 2, 203-233.

Jacauin, C., "Correlations Entre la Permeabilite et les Characteristiques Geometriques du Gres de Fontainebleau," Revue de 1'Institut Francais du Petrole (1964) 19, No. 7-8, 921-937.

Jensen, J. L., "A Statistical Study of Reservoir Permeability Distributions," Ph.D. dissertation, University of Texas, Austin (1986).

Jensen, J., Lake, L., and Hinkley, D., "A Statistical Study of Reservoir Permeability: Distributions, Correlations, and Averages," paper SPE 14270 presented at the 60th Annual Technical Conference and Exhibition of the Society of Petroleum Engineers, Las Vegas, September 22-25, 1985.

Johnson, N. L. and Kotz, S., Continuous Univariate Distributions, John Wiley and Sons, New York City (1970).

Jordan, D. and Tillman, R., El Dorado Micellar-Polymer Demonstration Project (Seventh Annual (Report), U.S. Department of Energy Report No. DOE/ET/13070-79 (June, 1982) 55-73.

Kenda11, M. and Stuart, A., The Advanced Theory of Statistics, Maclililan Pub. Co., New York (1979), Vol. 2.

Kova1, E. J., "A Method for Predicting the Performance of Unstable Miscible Displacement in Heterogeneous Media,: Soc. Pet. Eng. J. (June 1963) 145, Trans. AIME.

Khan, A. M., "An Empirical Approach to Water-flood Predictions," J. Pet. Tech. (May 1971), 565.

Kruskal, J. B., "Statistical Analysis: Transformations of Data," International Encyclopedia of the Social Sciences, MacMillan Co., Chicago (1968) 15, 182-193.

Lambert, M. E., "A Statistical Study of Reservoir Heterogeneity," M.S. Thesis, University of Texas at Austin, 1981.

Langeland, T. and Flotre, J., "Transformation, Interpolation, Regression Diagnostics, Graphical Displays and Q - Porosity Relations," Paper KK presented at the SPWLA Twenty-fifth Annual Logging Symposium, New Orleans, June 10-13, 1984.

Levorsen, A. I., Geology of Petroleum, W. H. Freeman and Co., San Francisco $(196 \overline{67)}$.

Liebelt, P. B., An Introduction to Optimal Estimation, Addison-Wesley Pub. Co., Reading, Mass. (1967). 
Matiisen, A. and Atwater, J., "An Overview of Computer-Enhanced Analyses of Core Data," Journal of Canadian Petroleum Technology (1984) 23, No. 3, 57-62.

Montaglou, A., and Wilson, J. L., "The Turning Bands Method for Simulation of Random Fields Using Line Generation by a Spectral Method," Water Resources Research, Oct. 1982.

Pottier, J., Jacquin, C., Marle, C. and Montadert, L., "Methodes et Moyens Pour l'Etude des Mlieux Poreux Naturels," Revue de 1'Institute Francais du Petrole, (1964) 19, No. 7-8, 872-900.

Schmalz, J. P. and Rhame, H. S., "The Variations in water Flood Performance with Variation in Permeability Profile," Producers Monthly (1950), 9.

Shinozuka, M., and Jan, C. M., "Digital Simulation of Random Processes and its Applications," J. of Sound and Vibration, 25(1), 1972.

Tootle, J. R., "The Prediction of We11 Productivity from Wireline Logs, McAllen Ranch Field," Paper H presented at the SPWLA Twentieth Annual Logging Symposium, Tulsa, June 3-6, 1979.

Tukey, J. W., "On the Comparative Anatomy of Transformations," Ann. Math. Stats. (Sept. 1957) 28, No. 3, 602-632.

Weisberg, S., Applied Linear Regression, John Wiley and Sons, New York (1980).

Warren, J. E. and Cosgrove, J. J., "Prediction of Water Flood Behavior in a Stratified System," Soc. Pet. Eng. J. (June 1964) 149, Trans. AIME, 228.

Warren, J. E., Skiba, F. F. and Price, H. S., "An Evaluation of the Significance of Permeability Measurements, J. Pet. Tech. (Aug. 1961) 739.

Wendt, W., Sakurai, S. and Nelson, P., "Permeability Prediction from Well Logs Using Multiple Regression," paper presented at the National Institute for Petroleum and Energy Research (NIPER) Reservoir Characterization Technical Conference, Dallas, April 29-May 1, 1985.

Wilson, D. and Hense1, W., "Computer Log Analysis Plus Core Analysis Equals Improved Formation Evaluation in West Howard-Glasscock Unit," Journal of Petroleum Technology, (Jan. 1978) 30, No. 1, 43-51. 
PART IV. HETEROGENEITY CLASSIFICATION

Part III gave the groundwork for a statistical classification of reservoir heterogeneity. A second way to classify heterogenejty is through the categories of geologic observation. Such observation ties nicely with statistics for it can provide a model upon which to begin the analysis.

This part presents the results of a geologic study on an eolian outcrop. The study is intermediate to two other goals: it will provide a basis for the heterogeneity classifications discussed in Lake et al. (1985), and it will be the basis for some rigorously realistic fluid simulations which will be performed in the third contract year. 


\section{DISTRIBUTION OF GEOLOGIC FEATURES IN EOLIAN SANDS}

With the passage of eolian facies modelling from infancy, criteria for bedform reconstruction and interpretation are beginning to be recognized and applied. Armed with such tools, ancient eolian sequences can now be identified with a high degree of confidence, and specifics of their morphology more sharply delineated. Details of eolian deposition are derived from observations of modern and ancient dune, interdune, and sand-sheet environments, but studies have relied heavily on theory; the internal structure of dry, sandy bedforms does not lend itself readily to observation, and the typically small fraction of the dunes actually preserved (Rubin and Hunter, 1982; Kocurek and Nielsor, 1986) restricts interpretation. The resultant models of eolian bedform reconstruction draw upon the two fundamental aspects of bedform morphology: the hierarchy of erosional surfaces that separate the successive units in a sequence (bounding surfaces of Brookfield, 1977), and the deposits themselves. By noting, on a unit-by-unit basis, the geometry and internal characteristics of both the bounding surfaces and the stratification it is theoretically possible to characterize the succession of bedforms represented by a particular eolian sequence.

As noted by Hunter and Rubin (1985), the tools exist whereby: (1) processes responsible for the development of bounding surfaces within a given sequence can be identified, (2) low-angle stratified deposits of dune aprons, interdunes, and sand sheets can be distinguished, (3) specifics of dune morphology can be outlined (i.e. the relative degree of curvature of the crestline determined, dune 
height and width roughly estimated, and where compound bedforms are implied, the trend of the superimposed dune relative to the primary bedform can be derived), (4) the role of secondary flow can be considered, from which generalizations concerning bedform dynamics might be made, and (5) where cyclic cross-bedding inferred to result from seasonal wind patterns is present, bedform migration rates can be estimated.

Components of the model of eolian bedform reconstruction have each been documented and tested, but there lacks an updated application of all the available tools to an ancient eolian sequence. Hence, the objective of this work: to test the degree to which current theory can reconstruct and interpret preserved eolian deposits.

Tools For Bedform Reconstruction and Interpretation - Within ancient eolian sequences cross-stratified sets are invariably separated from each other by subhorizontal bounding surfaces (Fig. IV-1). Present theory holds that first-order bounding surfaces (terminology of Brookfield, 1977) form primarily by dune migration and climb (Kocurek, 1981a, 1984; Rubin and Hunter, 1982, 1984; autocyc1ic hypothesis of Simpson and Loope, 1985). In contrast, regional bounding surfaces represent relatively isochronous deflated horizons that formed in response to "extra-erg events" such as climatic change, sea-level fluctuation, or erg migration (Loope, 1981, 1984; Kocurek, 1984; Talbot, 1985; super surfaces of Kocurek and Oakes, 1985). The water table or any overlying resistant horizon or crust may have served as base level of deflation. Bedforms probably 


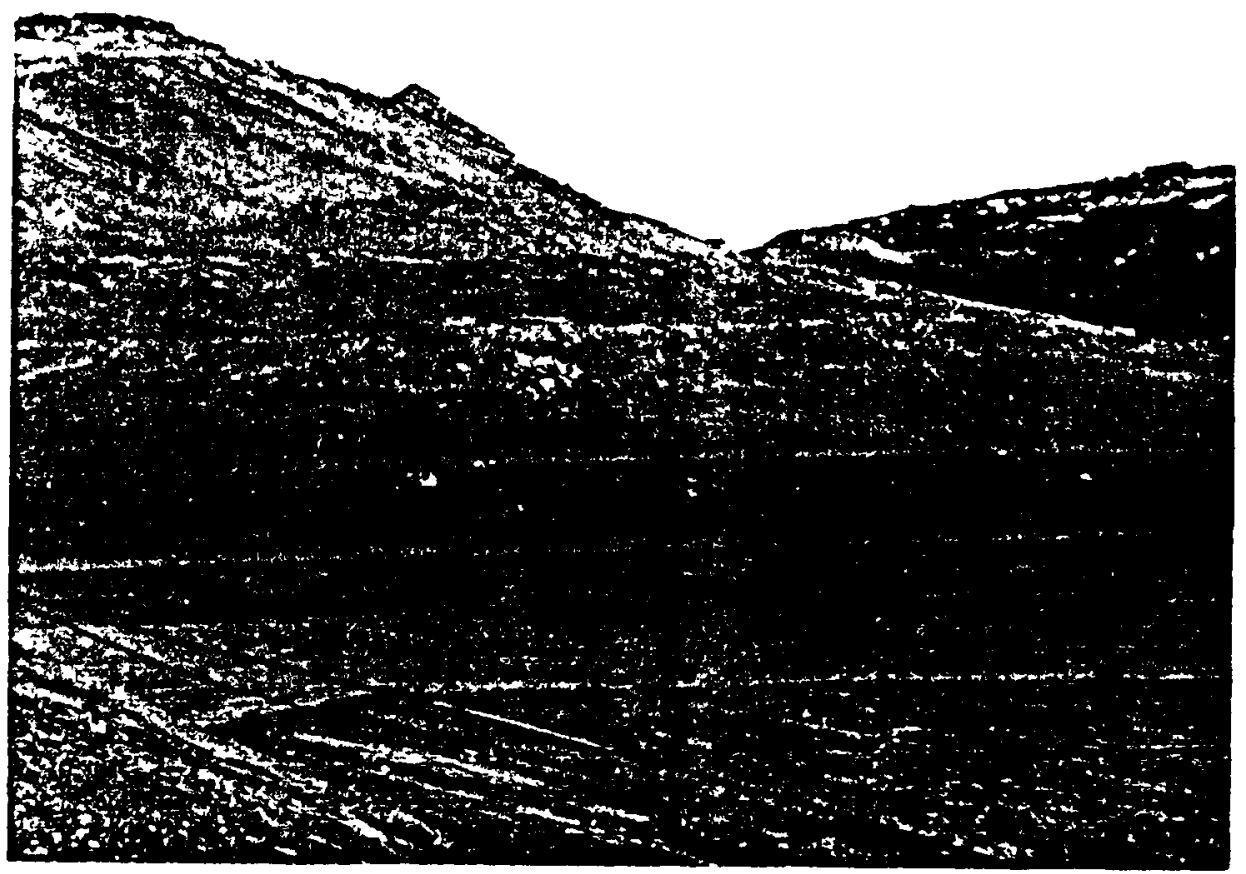

Figure IV-1. Subhorizontal first-order bounding surfaces separating cross-bedded Units of the study site. 
migrated across these surfaces at zero or slightly negative angles of climb (Rubin and Hunter, 1984). A complex sequence of amalgamated interdune deposits and lenses of cross-bedded dune sands has been interpreted by Simpson and Loope (1985) to represent a specific case where the angle of dune climb varied with a fluctuating sand supply.

Overlying extradune deposits would, of course, indicate a change in the conditions of deposition and concurrent development of a regional bounding surface (e.g., Blakey and Middleton, 1983; Driese and Dott, 1984). Evidence of long-term exposure may also be used to help identify regional bounding surfaces. A brief hiatus in sand-sea deposition and the concurrent development of a regional bounding surface are suggested by enriched zones of fines, deflation lag, evaporites (or indications of their former presence) immediately below the truncation surface, and paleosol development (Loope, 1984; Rubin and Hunter, 1984, Talbot, 1985). Unfortunately, these structures are not always preserved, nor does their presence absolutely disqualify a first-order bounding surface interpretation (Rubin and Hunter, 1984).

Because they develop in response to processes other than bedform migration, regional bounding surfaces do not show any directional relationship with the overlying strata. Relative to 'paleo-horizontal', regional surfaces should generally lie flat or dip slightly basinward. Within a particular eolian sequence, they should consequently all be oriented in roughly the same direction, barring major changes in basin configuration.

First-order bounding surfaces are suggested by a uniform cross-bed set thickness, and by the presence of thinly bedded interdune deposits (Kocurek, 1981a; Rubin and Hunter, 1982; Blakey 
and Middleton, 1983; Talbot, 1985). Trough-shaped first-order surfaces are generated by migrating three-dimensional bedforms (McKee, 1979c) or by scour pits that, as they migrate laterally along the base of a lee slope, produce a scalloped geometry (Rubin and Hunter, 1983). The relationship of overlying foreset dip direction to first-order bounding surface orientation is very complex and remains poorly understood. For example, fleld measurements may show surfaces dipping upwind, downwind, or lying flat, because dunes climb at very low angles with respect to the depositional surface, which does not necessarily correspond to horizontal. Further complications arise from the lack of studies relating dune dynamics to bounding surface orientation. Bedform-climb has been documented (e.g. Kocurek, 1981a, b), but present theory strictly applies only to transverse bedforms. If a directional relationship could be established, yet another criterion for distinguishing types of bounding surfaces, and possibly for interpreting bedform morphodynamics, might be found to exist.

Second- and Third-order Bounding Surfaces - Second- and third-order bounding surfaces are comparatively steep, less extensive, truncating surfaces that may or may not be present within any particular set of eolian cross-strata. As superimposed dunes migrate down or across the lee-face of a large compound bedform or draa, second-order bounding surfaces are generated and compound cross-bedding is deposited (Brookfie1d, 1977; Kocurek, 1981a; Rubin and Hunter, 1983).

In contrast, third-order or reactivation surfaces are found in both simple and compound cross-bedding, and develop in response to changes in wind velocity or direction or both (Brookfield, 1977; 
Hunter and Rubin, 1983). If fluctuating flow conditions occur with any sort of regularity (for example, seasonal wind variability) cyclic cross-stratification will result. The observed cyclicity may consist of a conformable alternation of wind-ripple, grainflow, and possibly grainfall deposits (Hunter, 1977; Kocurek, 1981a; concordant cyclic cross-bedding of Hunter and Rubin, 1983). Alternatively, localized scour may generate reactivation surfaces and 'compound' cyclic cross-bedding that resembles compound cross-strata deposited by migrating superimposed bedforms.

Hunter and Rubin (1983) present the most complete summary to date of the criteria by which second- and cyclic third-order bounding surfaces may be distinguished. Unless the bedform is highly sinuous, reactivation surfaces will develop along the entire width of the slipface, whereas the widths of second-order surfaces are limited by the dimensions of the superimposed bedforms, the subsets consequently appearing lenticular in transverse cross-section. In longitudinal cross-section, second-order surfaces are extensive relative to subset thickness because their length is a function of the migration distance of the superimposed bedforms. On the other hand, the length of reactivation surfaces is practically limited by the length of the reworked lee-face, and will appear much shorter relative to subset thickness. Evidence of periodically reversed winds likewise supports a fluctuating flow model and the presence of reactivation surfaces. Caution must be exercised, however, as features indicating reversed flow may also be produced by secondary currents generated on the lee-side of a compound bedform. 
Interpreting Low-angle Stratification - Low-angle $\left(\sim 215^{\circ}\right.$ eolian stratification (215 deg) may be deposited as sand-sheets, interdunes, or as dune aprons or plinths (Kocurek, 1985). Because sand-sheets form a distinct subenvironment typically at the erg margin, distinguishing these deposits carries important implications regarding ancient erg dynamics and morphology (e.g. Kocurek, 1981b; Ross, 1983; Porter, 1986). Guidelines for determining the depositional subenvironment of any low-angle-stratified sequence have been outlined by Kocurek (1985) and include the following key points:

(1) Sand-sheet deposits commonly occur at the base, at the top, or laterally along the edge of an eolian sequence. Interdune deposits overlie first-order bounding surfaces and alternate with dune deposits.

(2) The geometry of dune and interdune deposits vary predictably as a function of dune shape and migration patterns, whereas sand-sheets may occur as extensive deposits up to several meters thick.

(3) Factors that promote sand-sheet development, such as a high water table, surface cementation or binding, vegetation, and coarse grain size (Kocurek and Nielson, 1986, a), likewise inhibit dune development.

(4) Dune apron and plinth wind-ripple laminae dip up to $20-25^{\circ}$, show a marked parallelism within each package, and may include conformable grainflow and grainfall stratification. Conversely, sand-sheet and interdune deposits are gently-dipping (except where coarse-grained zibar deposits occur), typically irregularly laminated wind-ripple strata, that may show sedimentary structures reflecting moist or wet depositonal conditions. Finally, (5) interdune sands are bimodal 
and are typically more poorly-sorted than dune deposits (McKee and Tibbitts, 1964; Folk, 1968; Wilson, 1973; Ahlbrandt, 1979).

Also, a trend from dunes to zibars to granule ripples accompanies increasingly coarser grain size for eolian sediments. Zibars are slipfaceless, low-relief, coarse-grained bedforms common to sand-sheet and interdune corridor environments (Holm, 1960; Nielson and Kocurek, 1985).

Bedform Reconstruction Sensu Stricto - Dune Morphology - The character of the deposits left by different dune types is a complex, interrelated function of both bedform morphology and dynamics - the response of a dune to primary and secondary airflow patterns. Separate discussions of these two factors is therefore somewhat artificial, but at this early stage of understanding, is nonetheless warranted. As it stands, the model of bedform reconstruction sensu stricto supplies the tools with which crestline curvature, dune dimensions, and draa geometry can be approximated.

Crestline Curvature - Simple cross-bedded sets in an eolian sandstone may represent the basal portions of barchan, crescentic ridge, linear, star, blowout, or parabolic dunes (descriptive classification of Breed and Grow, 1979, differentiating Isolated barchans from crescentic ridges; McKee, $1979 \mathrm{~b}, \mathrm{c})$. Recognizing a particular morphology is basically an issue of determining the degree of crestline sinuosity and the number and relative orientation of slipfaces (McKee, 1979a; Ahlbrandt and Fryberger, 1982). 
The degree of curvature of the original bedform is reflected in bounding surface and interdune geometries, in the degree of foreset dispersion, and in the distribution of stratification types. Tabular or sheetlike interdune deposits imply migrating linear (i.e. straight-crested) bedforms, while lensoldal, broadly lenticular, and irregular, interdune deposits suggest barchan, crescentic ridge, and star dune shapes respectively (Kocurek, 1985). The degree of foreset dispersion across a single cross-set has long served as the classic criterion (Reiche, 1938; McKee and Tibbitts, 1964; Fryberger, 1979; McKee, 1979c). The spread of cross-strata dip directions is a very useful tool for determining crestline sinuosity, but determining the number of slipfaces using the same criterion may prove misleading, owing to the potentially complex nature of bedform dynamics (Rubin and Hunter, 1985). The final tool, the distribution of stratification types, is the single most useful criterion for estimating not only dune shape but also bedform dynamics.

The stratification styles present within a preserved set provide a map of the surface processes acting across the base of the original bedform (Hunter, 1977, 1981; Kocurek, 1981b; Kocurek and Dott, 1981). See Figs. IV-2 through IV-4. Grainflow cross-strata are deposited by avalanching down the active slipface of the dune. Wind-ripple strata occur in areas exposed to moderate wind stress on the stoss slope, on the flanks of crescentic dunes, and at the base of the lee-face as an apron. Thin wind-ripple laminae may also be found separating grainflow sets, marking brief periods of reworking between avalanche events (Kocurek and Dott, 1981). Finally, grainfall deposits represent fallout from suspension onto portions of the dune adjacent 


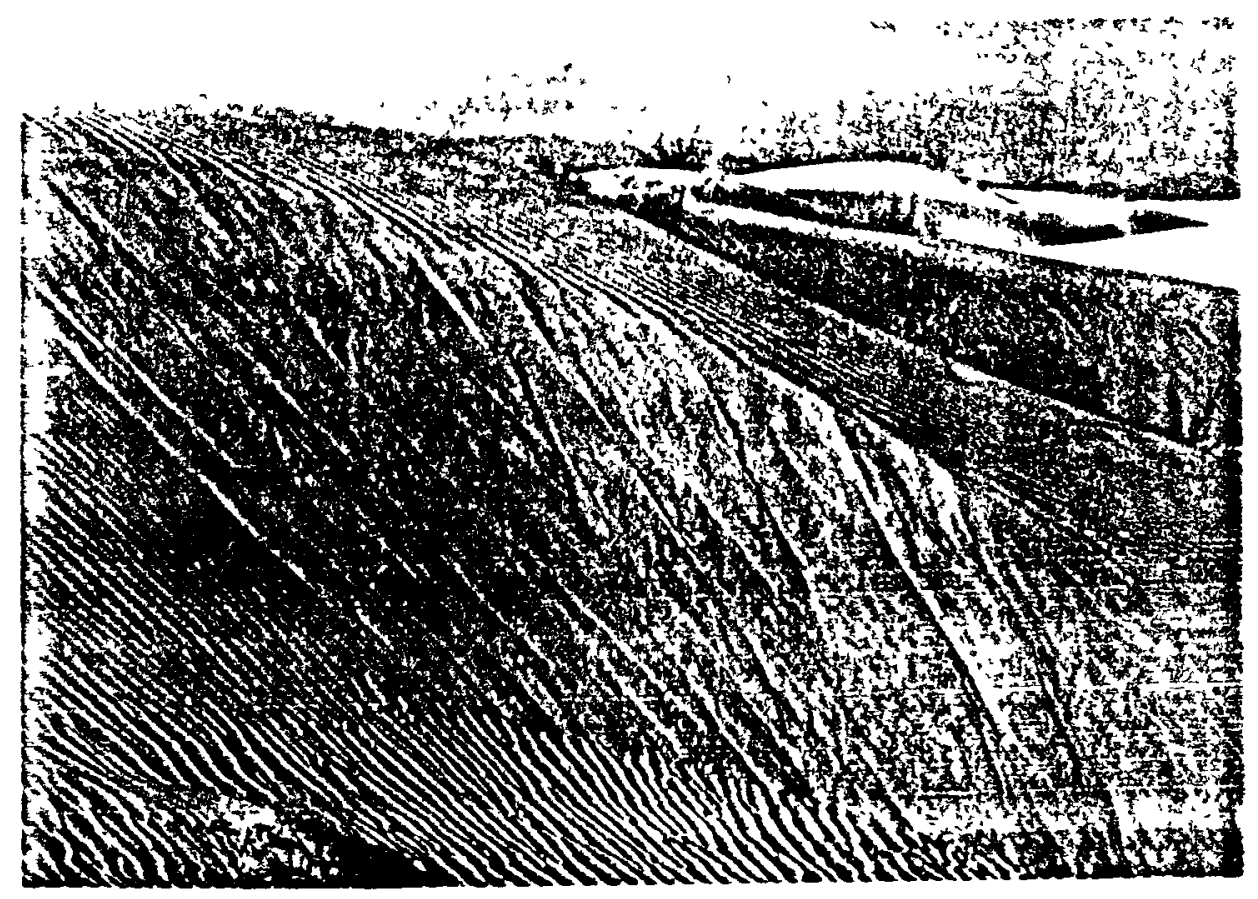

Figure IV-2. Grainflow and wind-ripple deposits on a small (approx. 6 m) slipface, Algodones Dune Field, California. The distribution of stratification types across an eolian bedform provides a map of local surface processes. 


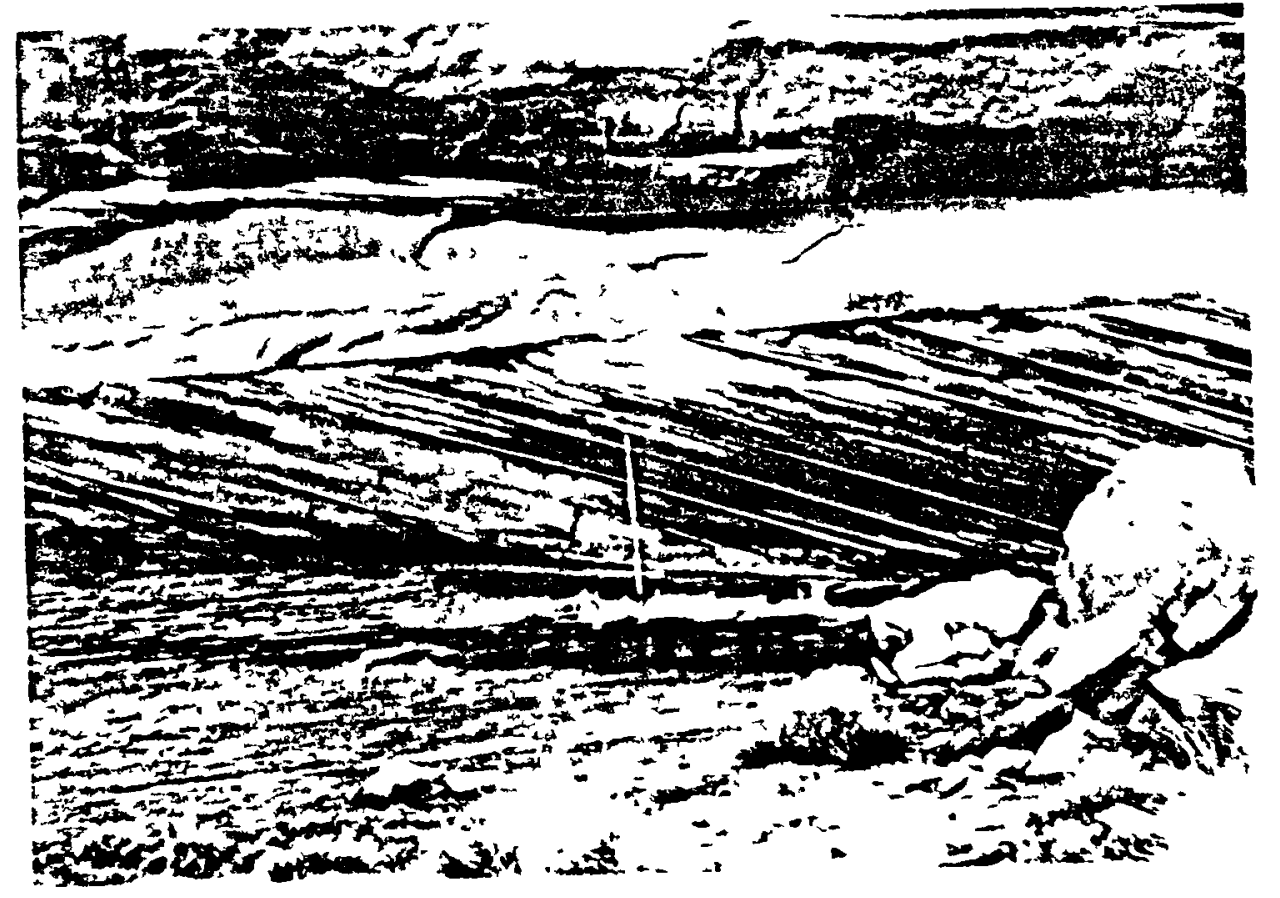

Figure IV-3. Grainflow stratification in the Page Sandstone at the study site. Packages of strata 20 to $40 \mathrm{~cm}$ thick are separated by thin, resistant wind-ripple laminae Table IV-2). Staff is marked in one-foot increments. 


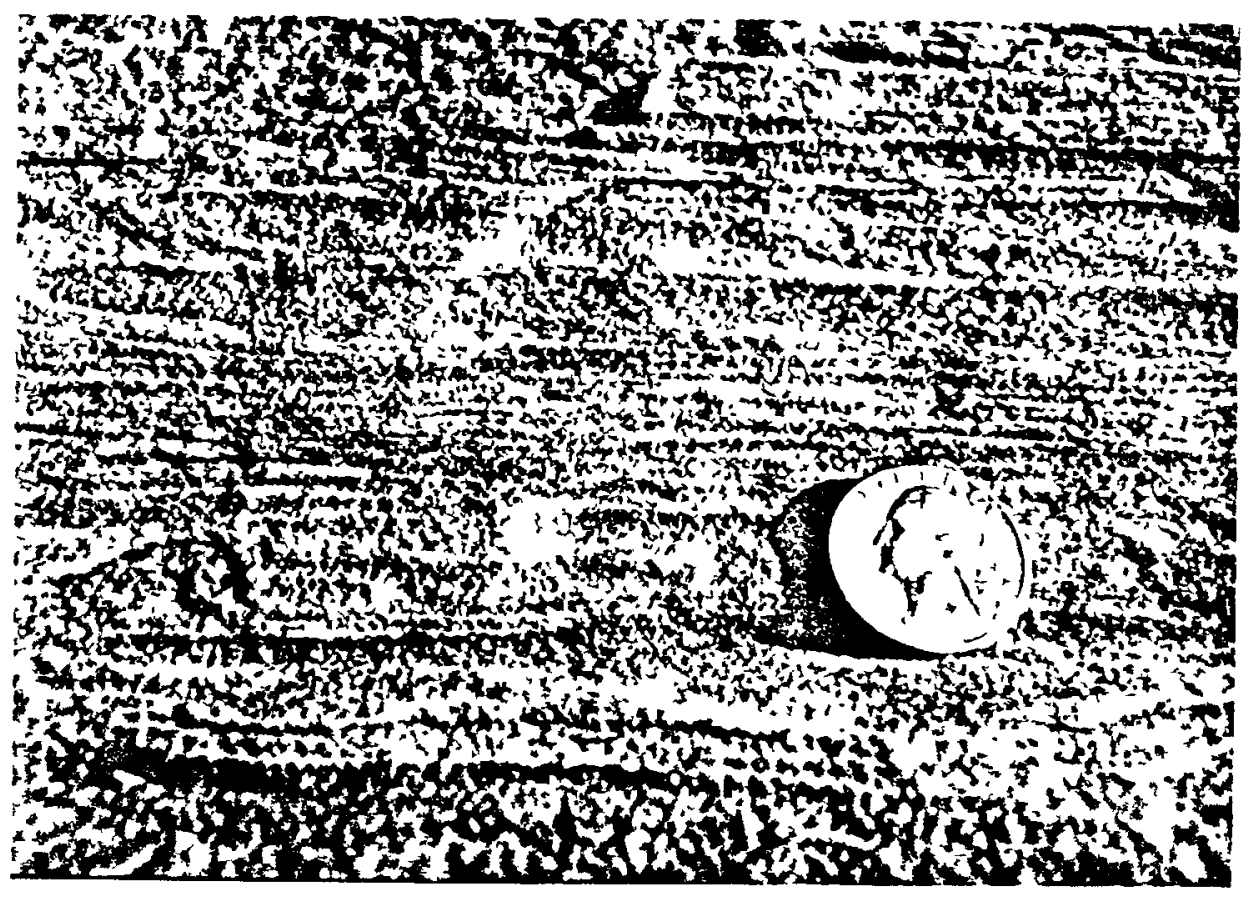

Figure IV-4. Wind-ripple stratification in the Page Sandstone at the study site. Note the inverse grading of thin windripple laminae. 
to the active slipface where wind stress was too weak for ripple growth. They are, however, generally limited to preserved deposits of smaller dunes and are rarely observed in the eolian record (Hunter, 1977, 1981).

Unless a lateral transition across a set from e.g. grainflow to wind-ripple deposits marks fluctuating flow conditions with time (concordant cyclic cross-bedding of Hunter and Rubin, 1983), a nonlinear dune shape may be invoked as an explanation. Curved bedforms exhibit complicated secondary flow patterns across their lee-face (Havholm, 1986; Hunter, 1981), productng an equally complex distribution of surface processes. For example, relatively protected areas along a slipface (such as the saddle of a crescentic bedform) may escape reworking, while adjacent peaks (or alternatively, flanks) might show a highly wind-rippled surface as grainflow and grainfall deposits are reworked. As these peaks and saddles (convex and concave portions of the lee-face respectively) migrate along the crestline in the course of bedform evolution (Bagnold, 1941; Tsoar, 1983), an alternating series of grainflow and 'reworked' grainflow sets should be deposited and possibly preserved.

Dune Dimensions - If the quality and the extent of an exposure are adequate, original dune width and wavelength may be directly measured; Kocurek (1981b) measured lenticular interdune deposits perpendicular to the inferred migration direction. He determined that crescentic bedforms of the Entrada Sandstone were approximately 80 meters wide. In the same study, the wavelength $(1.6 \mathrm{~km})$ was assumed equal to the distance, measured parallel to migration direction, between two 
successive first-order bounding surfaces observed to climb and intersect a horizontal "time line".

Unfortunately, such measurements cannot always be made. Assessing original dune dimensions therefore relies heavily on empirical relationships and qualitative observations. Work by Breed and Grow (1979) forms the framework with which dune width, length, and wavelength may be estimated. They show that modern dunes exhibit a predictable scale of proportions regardless of geographic location. If one of these dimensions can be determined independently, the others can be approximated by applying the empirical formulas that express these relationships (see Breed and Grow, 1979). Similarly, Wilson's (1972, 1973) observations of modern crescentic draas revealed a 1:15 ratio of height to wavelength. Applied to cross-bedded sets of the Entrada, Kocurek (1981b) estimated an original draa height of 110 meters. Modern draas range from 20 to 450 meters high (Wilson, 1973; Brookfield, 1984).

The direct relationship of dune height to individual grainflow lamina thickness provides a highly practical, independent means of estimating original dune height (Hunter, 1977, 1981; Kocurek and Dott, 1981). Grainflow lamina thickness remains fairly consistent across a cross-bedded set and is often easily measured. Although the exact function remains unknown, an empirical formula derived by Kocurek and Nielson (1986b) gives reasonable estimates of original dune height.

In cases where quantitative estimates cannot be made, the distribution of stratification types across a set can serve as a qualitative guide to ancient bedform size; larger dunes are indicated by a lack of grainfall deposits, wide grainflow sets as observed in 
transverse cross-section, and a relatively large scale distribution of stratification types (Hunter, 1977, 1981; Kocurek and Dott, 1981). In general, draas (compound or complex bedforms of Breed and Grow, 1979; McKee, 1979) are larger than simple dunes (Wilson, 1972; Kocurek, 1981b). Compound cross-bedding therefore also suggests greater primary bedform size.

Draa Geometry - The orientation of second-order bounding surfaces is controlled by the draa slipface and by the angle of climb of the migrating superimposed dunes. Because the angle of climb is typically small, second-order surfaces dip almost parallel to (and therefore approximate) the dip direction of the primary slipface. Cross-bedding orientation reflects the combined influence of the migration direction of the superimposed dune and gravitational forces working on the steep draa slipface. Foresets consequently dip at an oblique angle relative to both the primary and superimposed lee-face dip directions (Rubin and Hunter, 1983; Havholm, 1986). The orientation of the superimposed bedforms on the draa must therefore be determined indirectly. This trend is approximated by the line of intersection between the planes defining second-order bounding surface orientation and foreset dip direction (Rubin and Hunter, 1983). Correcting for this divergence gives a more accurate estimate of superimposed dune orientation.

As documented by Havholm (1986), draas usually exhibit marked morphological variation laterally across their lee-face. Figure IV-5 shows the lee-face of a transverse draa. Within one kilometer, areas lacking superimposed dunes occur adjacent to portions of the 


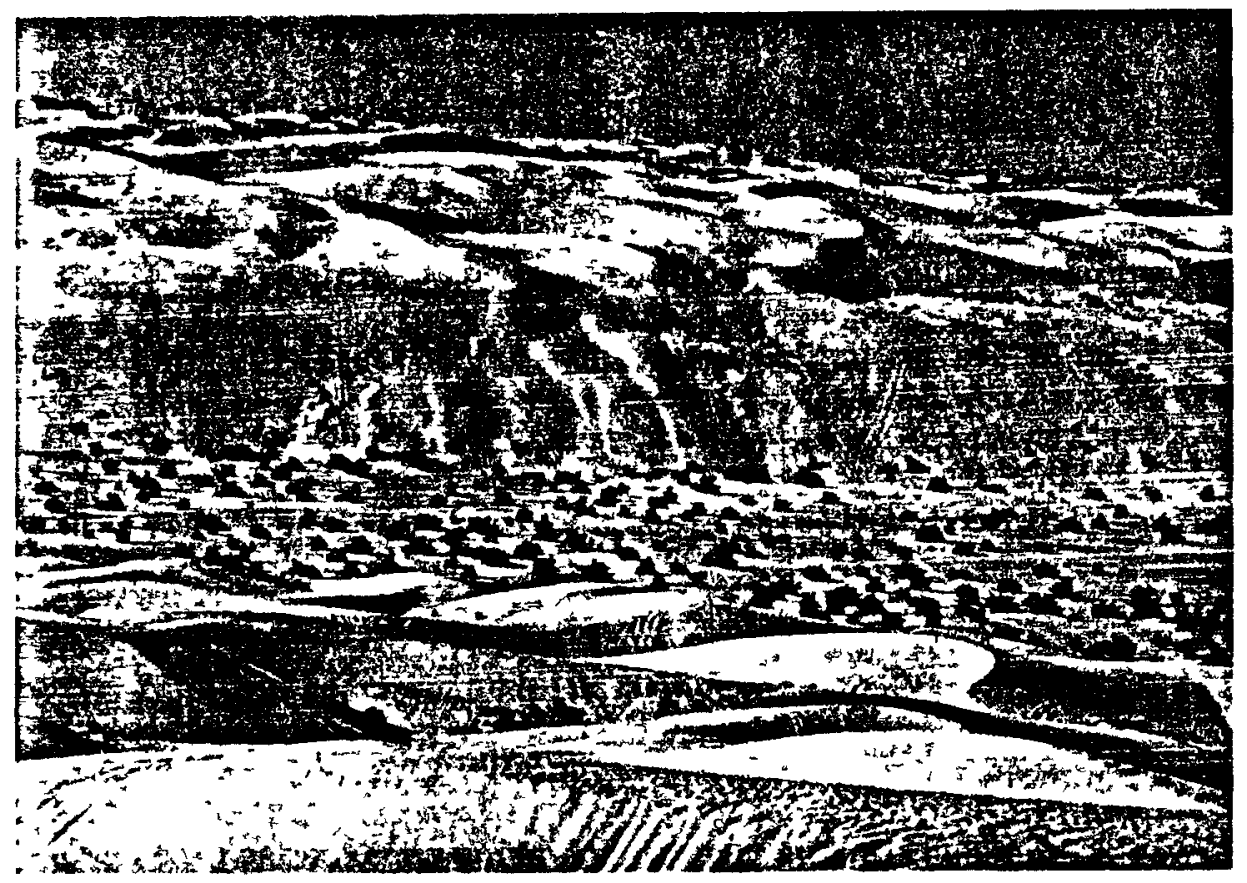

Figure IV-5. The lee-face of a transverse draa and a vegetated interdune corridor, Algodones Dune Field, California. Height of slipface is rough 1 y $30 \mathrm{~m}$. and is scarred by dune-buggy tracks. Areas dominated by avalanche deposition down the main slipface (left and right sides of the photo) commonly occur adjacent to areas of the lee-face where superimposed dunes migrate alongslope and obliquely downslope (center of photo). 
slipface where smaller dunes are migrating both alongslope and obliquely downslope (Havholm, 1986). Interpretations of ancient draa sequences have not yet considered this lateral variation. Indeed, the distribution of stratification types that would result from such a complex configuration might easily be misinterpreted as a series of simple dunes and draas of varying orientations. More studies of the internal structure and dynamics of draas are required before reconstructions can be made with any degree of confidence. Nonetheless, careful observations of bounding surface orientation and the details of stratification may render at least a gross interpretation.

Bedform Dynamics and the Relative Importance of Secondary Airflow The interdependence of dune shape and relative orientation to primary and secondary airflow patterns, constitutes the framework from which dynamic and depositonal models for the various dune types are derived. The morphodynamic classification proposed by Hunter (1983) and others is based on the angular difference between average dune trend and long-term resultant sand-transport direction. Initial boundary conditions established by the authors define transverse and longitudinal dunes as those oriented within 15 degrees perpendicular and parallel to the transport direction, respectively, with those in intermediate postions termed oblique. Complex flow across the lee-face of draas justifies separate consideration of compound bedforms. Details of stratification are the key tools by which these four dune types might be recognized in ancient sequences. Bounding surface orientation relative to foreset dip direction for different 
dune types has not been studied, but theoretically, a useful relationship should exist.

Transverse Dunes - Transverse dunes develop and migrate in response to unimodal wind regimes (Fryberger, 1979; Wasson and Hyde, 1983). Because their crests are perpendicular to a primary wind direction that exhibits low directional variability, secondary airflow across the lee face consists primarily of weak, variable back-eddy currents (Hunter, 1981). As a result, transverse dunes do not have significant wind-rippled aprons at the base of the active slipface. For reasons as yet unknown, straight-crested transverse bedforms are not a stable configuration. To some degree, all transverse dunes are crescentic or barchanoid (Breed and Grow, 1979; Hunter, 1981; Rubin and Hunter, 1985). Curve width remains fairly constant along a transverse crescentic ridge, but varies between dunes of different ergs. Local areas of weak, variably directed, secondary airflow will consequently develop on the lee face of these curved bedforms (Hunter, 1981).

As a result of the primary and secondary airflow patterns briefly described above, transverse dunes should deposit units: (1) that reflect a unimodal wind regime, including a relative absence of both reactivation surfaces and oppositely dipping slipface deposits, (2) that consist primarily of grainflow deposits extending to the base with minimal apron development, although wind-rippled flank deposits may be present, (3) that show evidence of crestline curvature, including a relatively broad spread of strata dip directions (Hunter, 1981; Rubin and Hunter, 1985), and (4) whose first-order bounding surface can be shown to dip upwind relative to the depositional 
surface (Kocurek, 1981a). Because it is relative to the depositional surface and not to 'paleo-horizontal', the observed dip direction of the first-order surface may parallel or may oppose the migration direction (which equals the foreset dip direction on transverse dunes), or it may rest flat.

Oblique Dunes - A dune crestline oriented obliquely to the resultant sand-transport direction may reflect any one of a number of conditions. The bedforms may be out of equilibrium with the local wind patterns, or alternately, may represent a stable configuration that is maintained by secondary flow. Lateral variations in dune height may rotate a dune to an oblique position by producing local changes in wind speed and direction (Rubin and Hunter, 1985; Havholm, 1986). Variable wind patterns may also effect an oblique orientation. Hunter and others (1983) observed that oblique dunes in Oregon are oriented exclusively by the stronger component of a bimodal wind regime. Winds blowing from the other prominent direction are too weak to control dune trend, but are persistent enough to influence the resultant sand-transport direction.

By virtue of their non-transverse orientation, the lee faces of oblique dunes are characterized by alongslope-directed secondary flow, which strengthens as primary winds intersect the crest at angles approaching parallel (Tsoar, 1983; Rubin and Hunter, 1985; Fig. IV-6). The strong component of alongslope flow locally diverts the sand-transport direction and reworks slipface deposits to produce a thick wind-rippled apron. Any morphological features present on the 


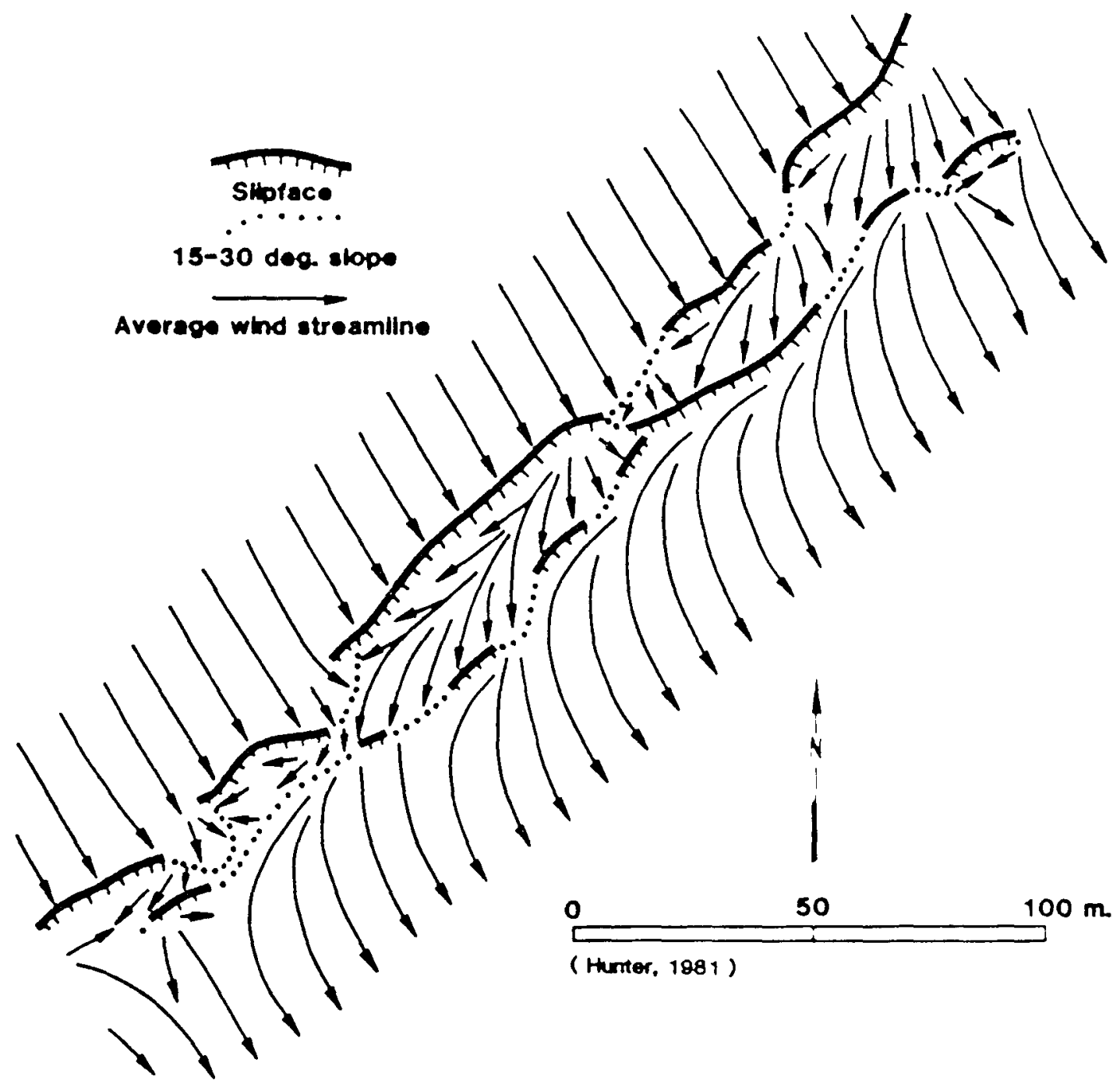

Figure IV-6. Primary and secondary airflow across an obliquelyoriented dune, Oregon coast, July 1978. Hunter (1981) measured wind directions both directly and indirectly, by noting wind-ripple orientations. Note the dominant alongslope component of secondary airflow across the dune's lee face. 
lee face of an oblique dune (e.g. scour pits, superimposed bedforms) should migrate preferentially alongslope (Rubin and Funter, 1985). Finally, oblique dunes tend to show straighter crests than transverse forms because secondary flow reworks the slipface (Hunter et al, 1983) .

Alongslope-directed secondary flow figures prominently in dictating the character of the deposits left by migrating oblique dunes. Reactivation surfaces and other indicators of a variable wind regime may be comparatively common, although strata should exhibit a unimodal dip pattern. Wind-ripple-stratified apron deposits comprise most (if not a11) of the preserved remnants, and indications of crestline sinuosity should generally be absent. For example, the straight-crested morphology of oblique dunes relative to transverse forms results in a comparatively tight distribution of cross-bed dip directions (Hunter et al, 1983). Finally, the relationship of first-order bounding surface orientation to foreset dip direction for oblique dunes has not been documented, but it is not an unreasonable conjecture that an angular divergence exists.

Longitudinal Dunes - Longitudinal dunes parallel the long-term sand-transport direction and are generally associated with variable wind regimes (Hunter et al, 1983; Fryberger, 1979; Tsoar, 1983). Morphologies range from straight-crested forms typified by the "seifs" of Australia (Folk, I971) to more sinuous shapes (e.g. Iongitudinal dunes of Algodones dune field; Nielson, 1986).

Considering their common occurrence in modern ergs, anomalously few eolian sandstones are interpreted as having been deposited as 
longitudinal dunes. Although some are non-depositional features (for example, helical flow has cut into fluvial sediments to form the siefs of Australia; Folk, 1971), it is likely that their deposits are not being recognized because the original criteria for identification are inaccurate (Rubin and Hunter, 1985). Recent studies have, however, shaped a refined model of stratification that should help to identify preserved remnants of longitudinal bedforms. Rubin and Hunter (1985) argue that longitudinal dunes must migrate laterally, unless seasonal sand-transport directions perfectly balance against a long-term transport direction that is exactly parallel to the dune crest. Observations by others show that alongslope-directed flow commonly reworks the slipfaces of longitudinal dunes to produce a thick, well-developed, wind-rippled apron (Hunter, 1981). Finally, complex airflow patterns across many longitudinal bedforms result in an equally complex distribution of surface processes (Nielson, (1986).

From these contentions, it follows that typical longitudinal dune deposits are composed primarily of moderately to gently dipping wind-ripple strata that exhibit a unimodal pattern of cross-bed dip directions (Rubin and Hunter, 1985). Trenches of longitudinal dunes in the Algodones dune field confirm, at least locally, a unimodal distribution (Nielson, 1986). Nonetheless, where sinuous crests and /or highly variable wind patterns are implied, a complex distribution of stratification types and orientations occur, and reactivation surfaces might be common (Nielson, 1986). As with oblique dunes, the directional relationship between first-order bounding surface and cross-stratification is not known, but theory 
suggests a slight angular divergence.

Draas - Because of their larger size and greater complexity, draas behave differently than their simple counterparts. A study by Havholm (1986) of the morphodynamics of a transverse draa reveals two details that should be kept in mind when reconstructing and interpreting ancient eolian deposits. Transverse draas (i.e. compound or complex bedforms that trend normal to the resultant sand-transport direction; Hunter and others, 1983) associated with variable wind regimes may exhibit a well-developed component of lee-side flow that parallels the main crest. Aerodynamically then, these draas more closely resemble simple oblique dunes than they do transverse forms (Havholm, 1986). In a compound cross-bedded sandstone, evidence that superimposed dunes systematically migrated alongslope does not, therefore, automatically eliminate a transverse draa interpretation. Secondly, Havholm notes a possible relationship between dune height and the presence or absence of a basal apron. The great height of some draas may effectively limit the amount of sand supplied to the lee face, thereby restricting apron development. Moreover, wind velocities in the lee of large draas are considerably weaker, which would likewise prevent reworking of slipface deposits into wind-rippled aprons. Such observations in modern environments emphasize both the complexity of eolian depositional environments and the limited interpretive resolution of ancient sequences.

Estimating Bedform Migration Rates - When cyclicity observed in a cross-bedded sandstone can be attributed to seasonal fluctuations, a 
quantitative estimate of the annual migration rate of the ancient bedforms may be made (Hunter and Rubin, 1983). Care should be taken to assure the reasonableness of an estimate; measurements taken in modern eolian environments indicate that large dunes migrate slowly typically a few tenths of a meter per year (Wilson, 1973; Hunter and Rubin, 1983; Havholm, 1986). Factors that control the rate of dune advance include prevailing wind velocities and variability, as well as dune size, shape, and relative orientation.

The Study Site Location - The study site is located in the Glen Canyon National Recreation Area near the town of Page, Arizona (Fig. IV-7). A knob of weathered sandstone approximately 36 meters high, extending over a $0.1 \mathrm{~km}^{2}$ area, was specifically chosen for its unobstructed, three-dimensional exposure (Fig. IV-8). Deposits of the Page Sandstone compose the knob and nearby buttes and mesas. The type section for the Page is found immediately adjacent to the study area on Manson Mesa. Comparing the height of the knob with the total thickness of the type section, the uppermost 18 meters of Page Sandstone appears to have been eroded from the study site.

Stratigraphic Setting of the Page Sandstone - The Page Sandstone was first described by Peterson and Pipiringos (1979) as a cliff-forming, cross-bedded, quartzose sandstone exposed in southern Utah and northern Arizona. Tabular- and wedge-planar cross-stratified sets 1 to 6 meters thick are the predominant features of the formation, 


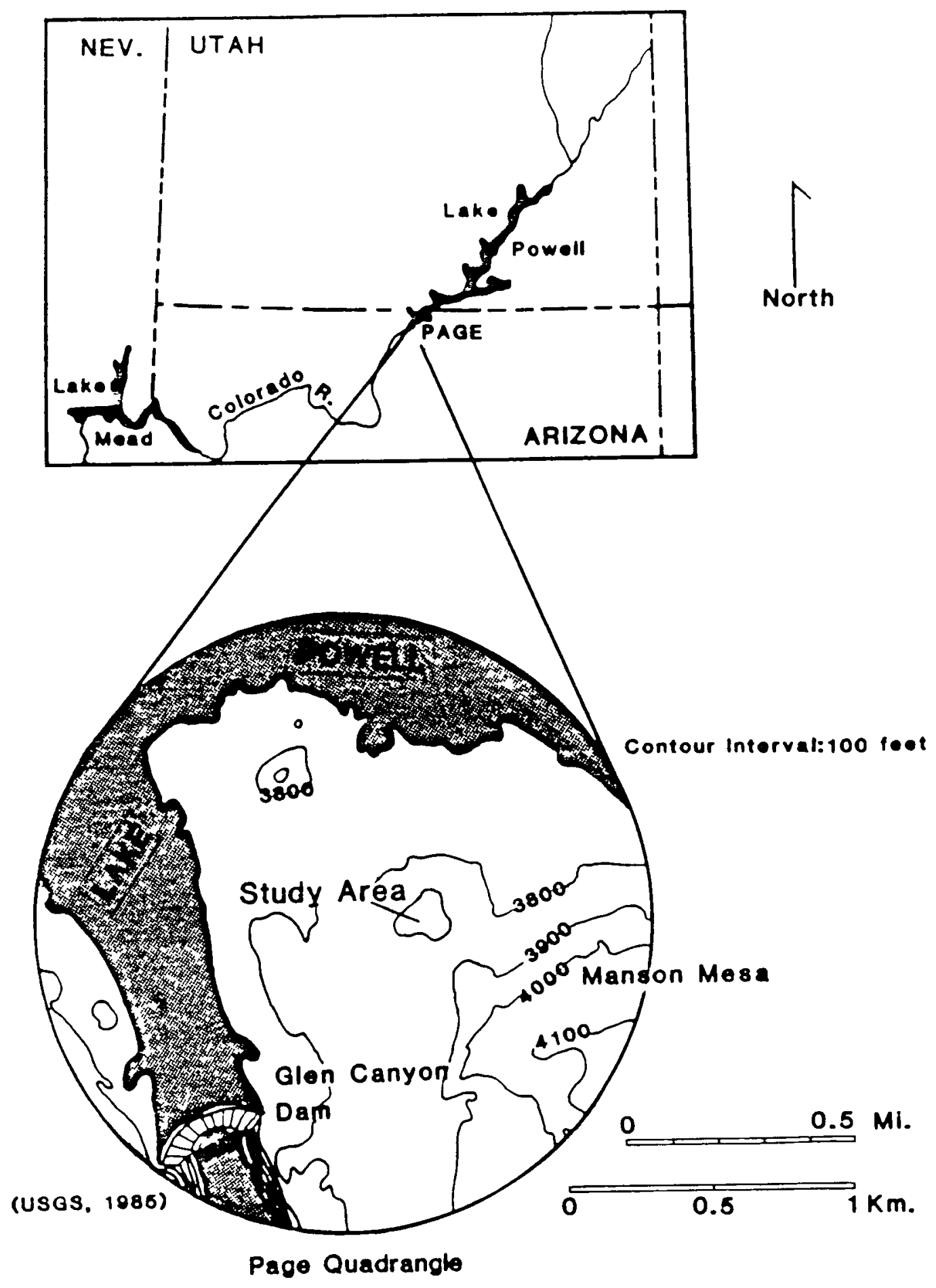

Figure IV-7. Location of the study site in northern Arizona. 


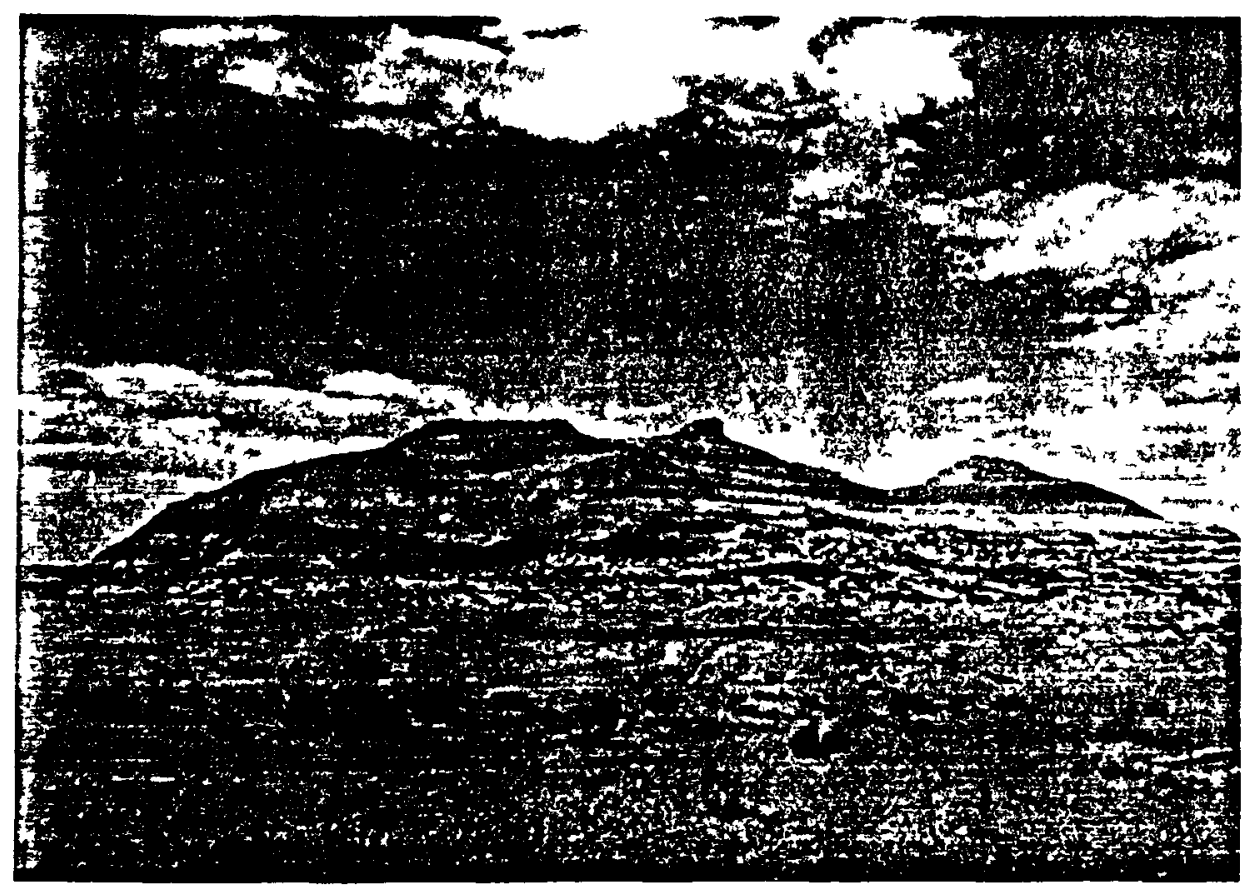

Figure IV-8. The southern side of the study site. Dark red deposits are units AlO and All - the uppermost units of the A-complex (Table IV-1). 
although trough-cross-bedding, horizontal lamination, and interbedded siltstones also occur (Blakey et a1., 1983).

The J-2 unconformity (also known as the chert-pebble unconformity) separates the Early Iurassic Navajo Sandstone from the overlying Middle Jurassic Page and the Middle to Upper Jurassic Carmel Formations. Extending across the Western Interior of the United States, $\mathrm{J}-2$ is marked by abundant chert pebbles and by dessication features that exhibit a polygonal plan-view geometry (Pipiringos and O'Sullivan, 1979; Kocurek and Hunter, in press; Fig. IV-9). Up to 11 meters of relief on the surface has been observed north of the study area. Smaller-scale irregularities of a meter or less also occur, suggesting that the eroded top of the Navajo was fairly well lithified when the sands of the Page were deposited (Peterson and Pipiringos, 1979).

To the northwest, sandstones, siltstones, and evaporites of the Carmel Formation interfinger with the Page Sandstone. The Judd Hollow tongue of the Carmel extends into the Page as far southeast as the town of Page in the vicinity of the study area (Caputo, 1980; Blakey et a1., 1983).

Paleogeographic Setting of the Page Sandstone - Middle Jurassic paleogeography of the Colorado Plateau consists of shallow marine conditions to the north and west, and sabkha and erg environments inland to the southeast (Blakey et a1, 1983; Fig. IV-10). The area was located in the trade-wind belt (between 10 and 20 deg. north latitude), and so was probably characterized by hot, dry conditions 


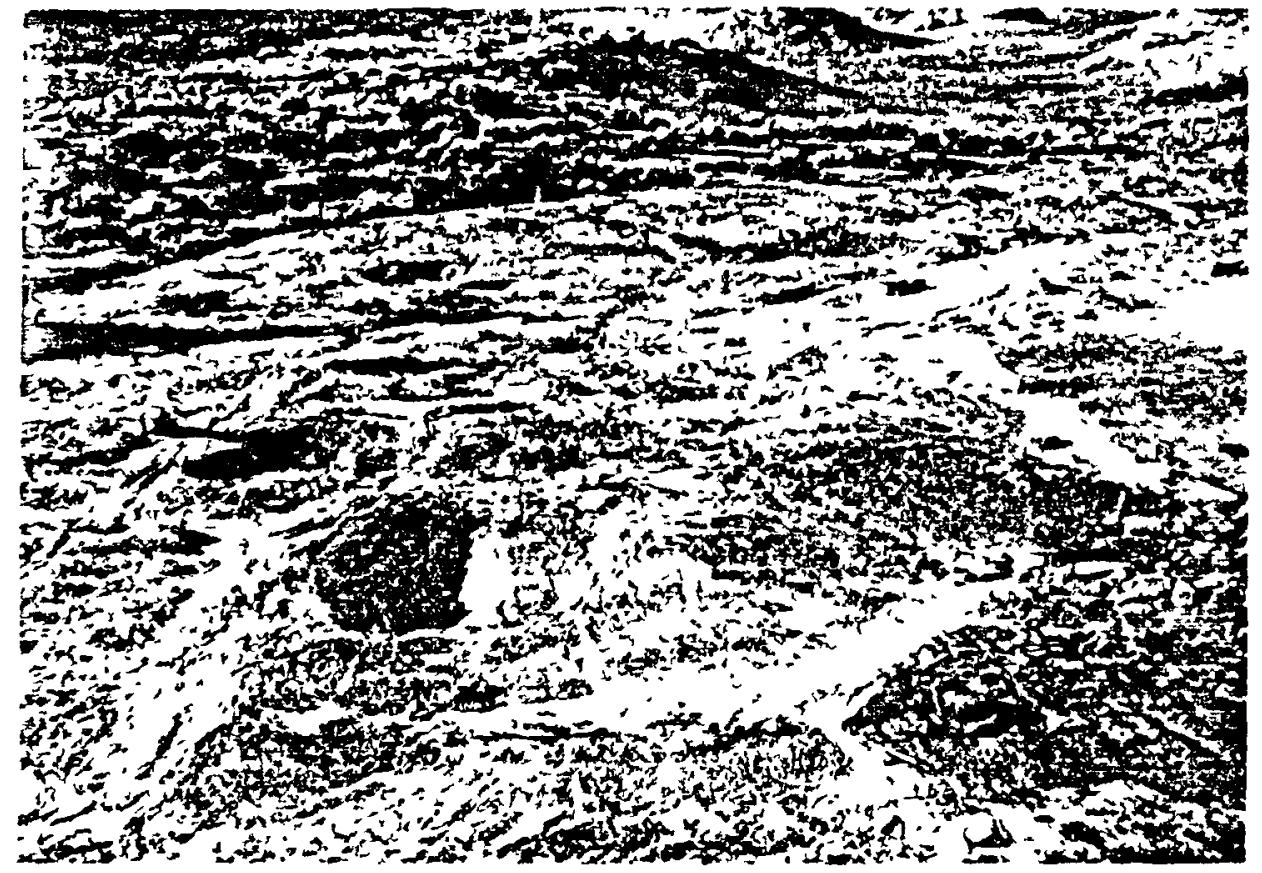

Figure IV-9. The J2 surface adjacent to the northwest wall of the study site. The distinctive polygonal pattern produced by dessication structures averages 2 meters wide (Kocurek and Hunter, 1986). 


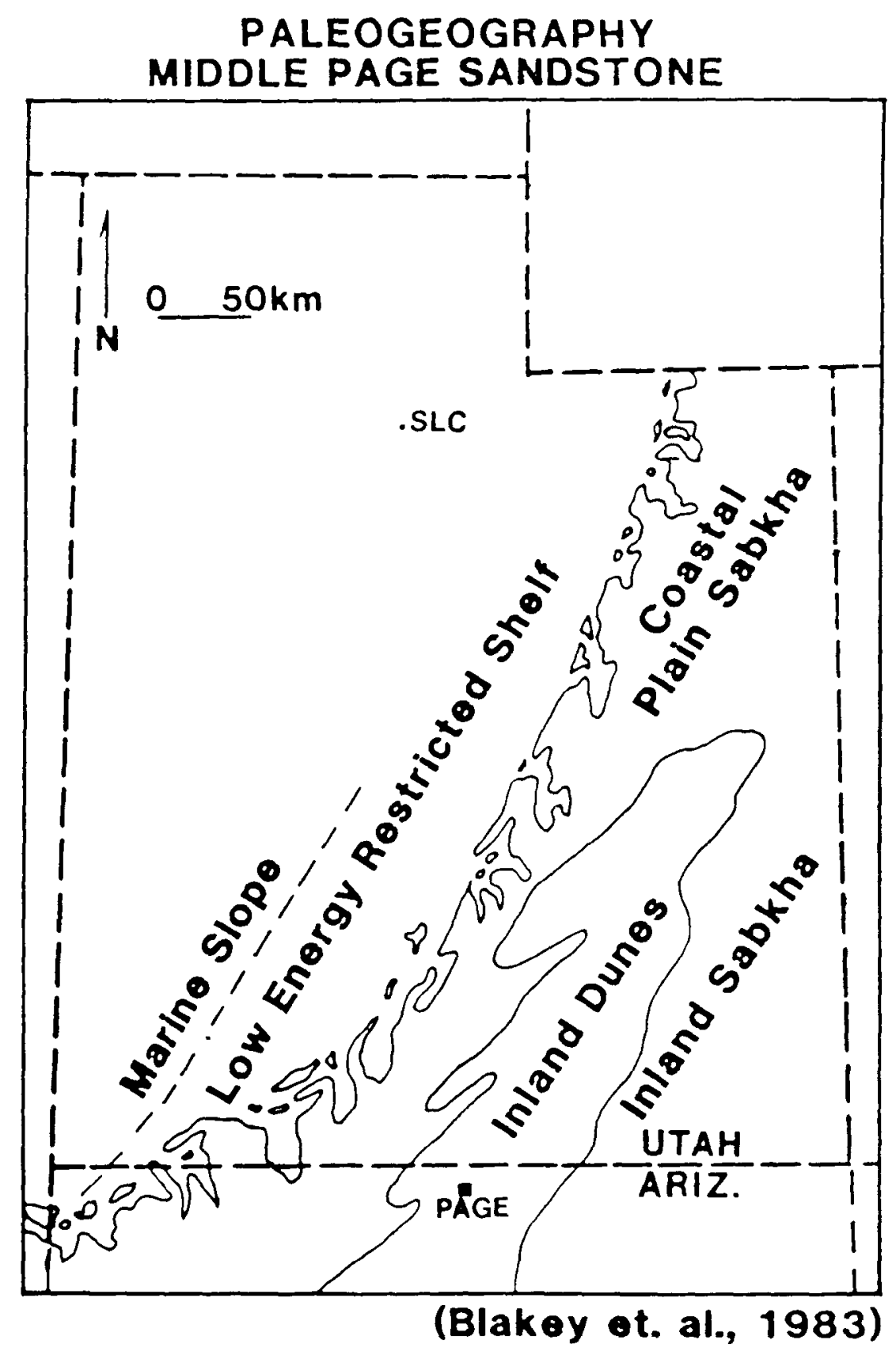

Figure IV-10. Paleogeographic setting during the Middle Jurassic, during Page deposition. Study site is located near the town of Page. From Blakey et a1. (1983). 
and prevailing winds from the north and northeast (Poole, 1962; locurek and Dott, 1983).

A minor transgression earlier in the Jurassic reduced the amount of sand supplied to the regionally extensive Navajo erg, forcing it to retreat southward. The Page Sandstone represents a northward progradation of this sand-starved Navajo erg (Kocurek and Dott, 1983). Sands of the Page were deposited atop a deflated tidal flat as sand-sheets, dunes, and interdures. In the course of Page deposition, the study site continued to be located only a short distance from restricted marine or sabkha conditions. Polygonal fractures, formed by thermal contraction of exposed evaporite surfaces, occur along the J-2 surface and locally along four other horizors within the Page Sandstone, Fig. IV-11. Moreover, extra-erg deposits occur in the general study area as thin beds of brown sandstone, mudstone, and limestone, recording minor sea-level fluctuations and brief periods of Page erg retreat (Blakey et al, 1983).

Method of Study - The study knob was mapped using a Lietz SDM3E electronic tacheometer, a surveying instrument that measures travel times of an oriented laser beam to calculate absolute and relative distances. Five-hundred eighty-eight points along first- and second-order bounding surfaces were marked for surveying. Each point was then assigned three coordinates defining its relative position north-south, east-west, and vertically $x, y$, and $z$ coordinates, respectively, Fig. IV-12. Coordinates calculated and displayed by the 


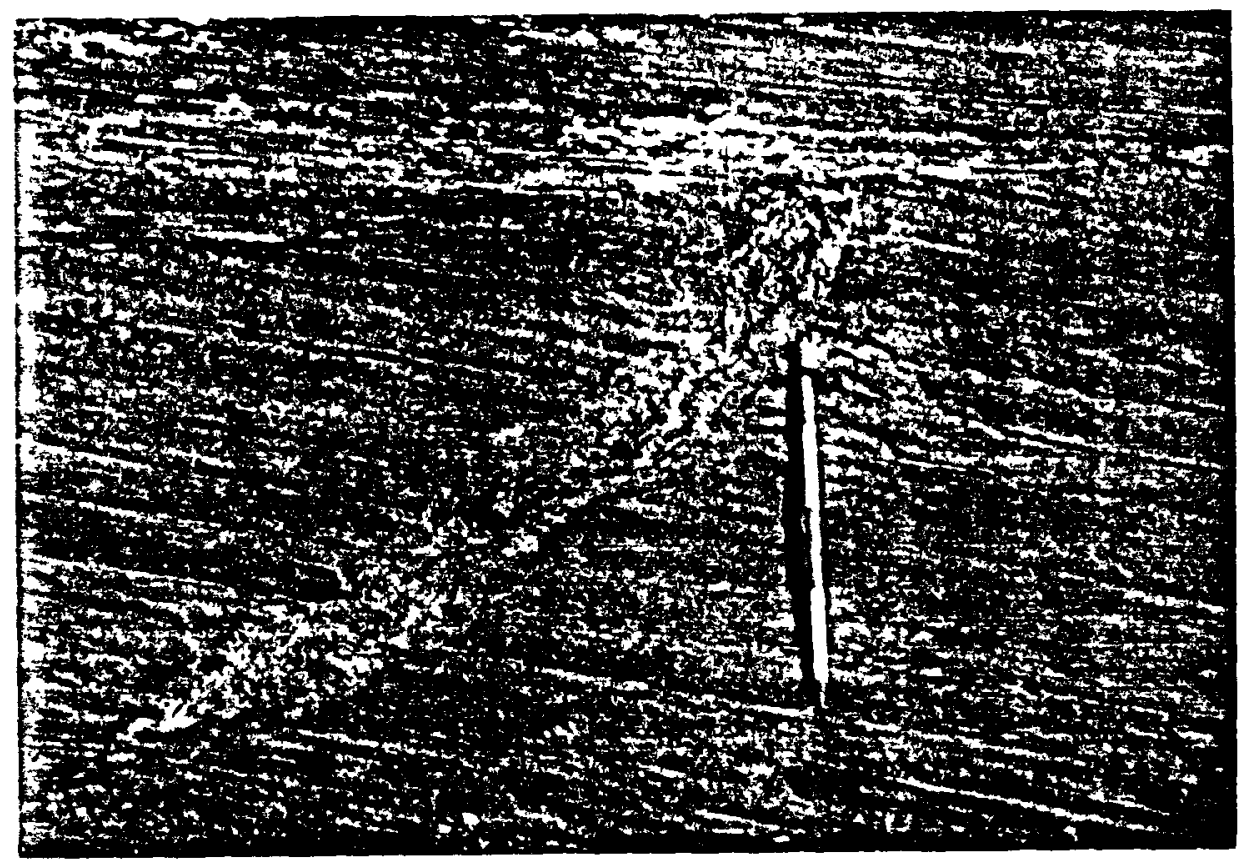

Figure IV-11. Cross-sectional view of a polygonal fracture, Page Sandstone. The fracture is found at the study site along the bounding surface underlying deposits of unit C13 (Table IV-3). Staff is marked in one-foot increments. 
tacheometer are relative to the instrument's location. Because the tacheometer had to be set up at numerous sites around and on top of the knob, the measured vaiues had to be adjusted to a single, artitrary reference point. This was achieved by 'shooting' some o1 the points trom more thai one racheorieter site. Ar estimated measurement error of 10-20 centimeters was determined by roting the ciscrepancies that arose while normalizılig the data set.

A pianimetric base map showing bounding surfaces was constructed by plotting the xy coordinates at a reasonably large scale and comecting the appropriale points (Fí. IV-13). For cross-sectional views, xy coordirates were projected from their planinetric position onto ore of five linear trends that together approximate the shape of the knob. Their positions along the lines onto which they fali define a single $x y$ coordinate, which was then plotted against the original $z$ (height) cocrdinate. Accurate cross-sectional base maps oi first- and second-order bounding surfaces result by again connecting the appropriate points.

During subsequent field visits, thest bast maps were used to locate each of 718 strike and dip measurement sites, and to sketch in the distribution of the stratification types and reactivation surfaces. Before the stratification could be added to the final maps, the apparent dips in the plane of the appropriate cross-section had to be calculated for each mapped measurement. The resultant cross-sections, showl in Fig. IV-14 through IV-17, are very accurate quantitative reconstructions. In some areas where bounding surface geometry appears distorted, data points were taken farther up or down 


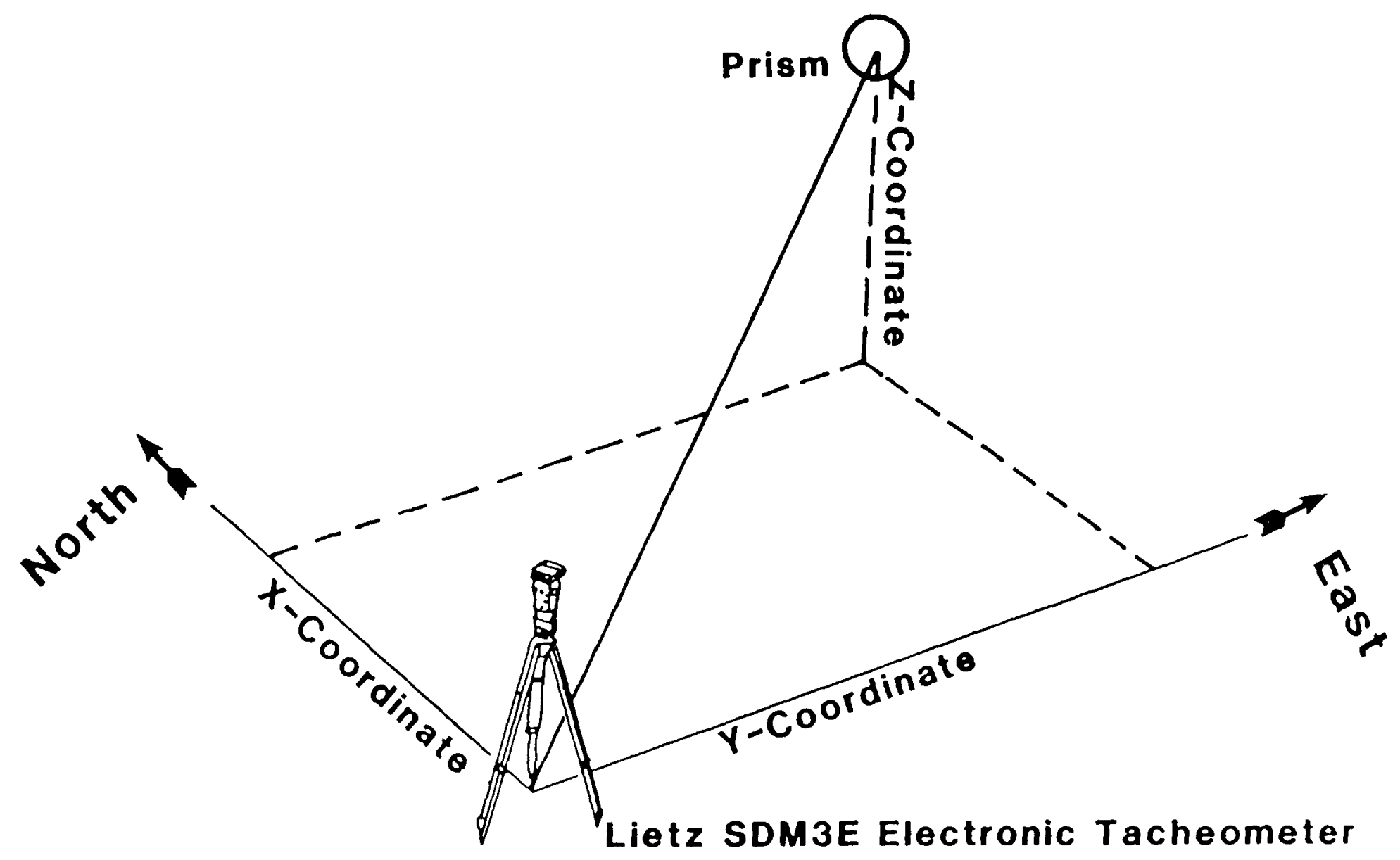

F1gure IV-12. Set-up of the Leitz tacheomter, and its relationship to the coordinates which it calculates. 
- Data points

- First- and second-order bounding surfaces

.... Polygonally fractured surfaces

-... $\mathrm{J} 2$ unconformity 


\section{NORTHWEST WALL (N48E)}

\section{EXPLANATION}

- Data points

First- and second-order bounding surfaces

Reactivation surfaces

Reactivation surfaces

Grainflow stratification

Polygonally fractured horizon

7

Polygonally fractured horfzons

Ing surfaces

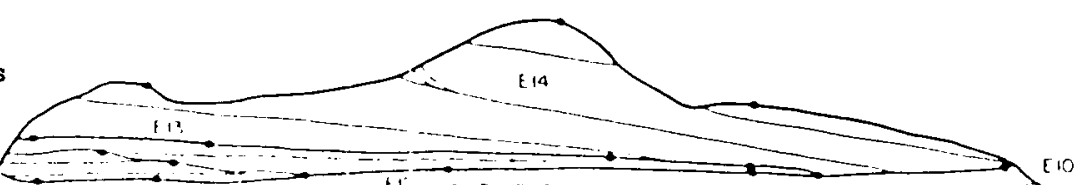

tion

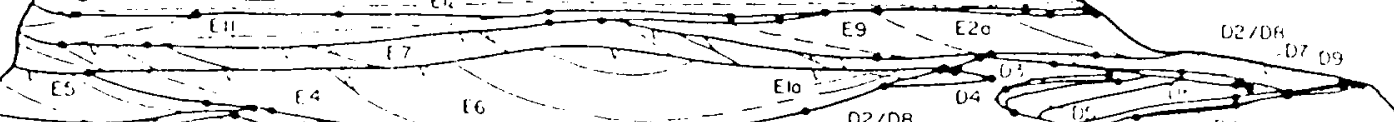
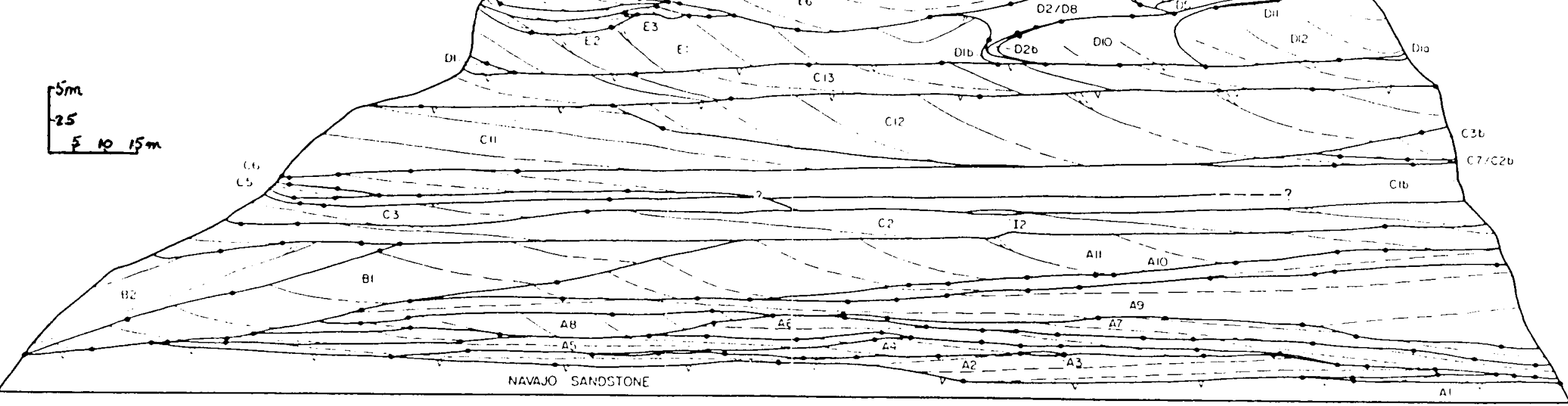

Figure IV-14. A reconstruction of the northwest side of the study knob. The key for this figure also applies to the other cross-sections, Fig. IV-15 through IV-17. 


\section{SOUTHWEST WALL (N24W)}

\section{EXPLANATION}

\section{- Data points}

First- and second-order bounding surfaces

Reactivation surfaces

Reactivation surfaces

Grafiflow stratification
Wind-ripple stratification

Polygonally fractured horfzons

SOUTH WALL (NGOE)
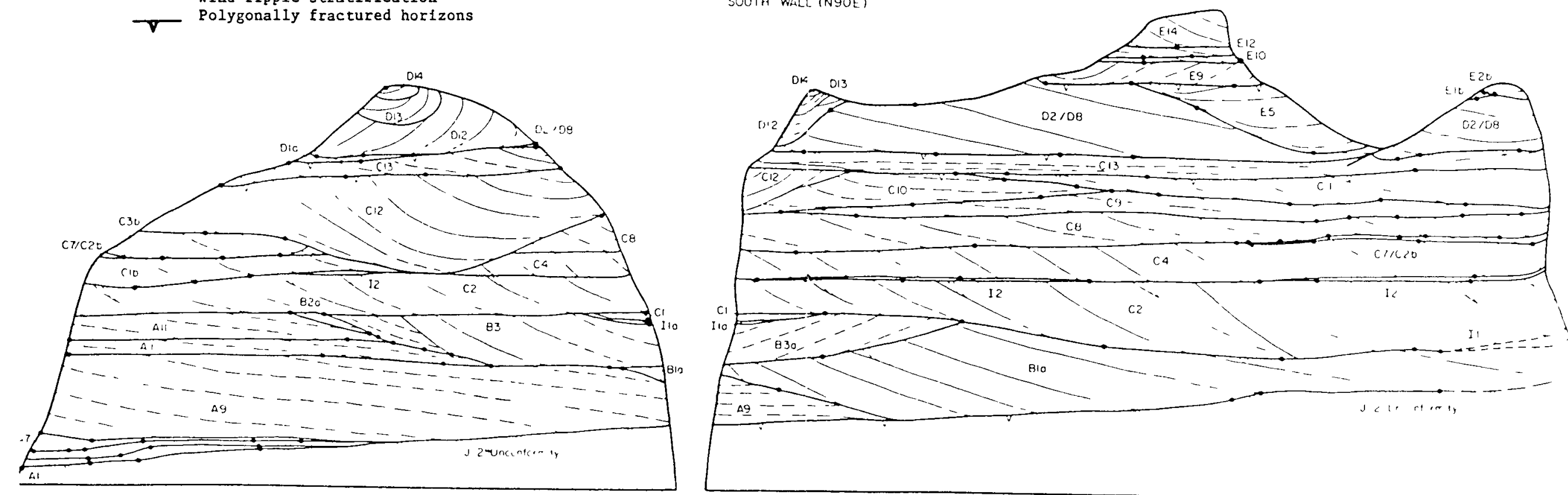

Figures IV-15a, b. Reconstructions of the southwest and south sides of the study knob. 
SOUTHEAST WALL (N41E)

EXPLANATION

- Data polnts

First- and second-order boundiag surfaces

Reactivation surfaces

Grainflow stratification

- - Wind-ripple stratification

$\checkmark$ Polygonally fractured horizons

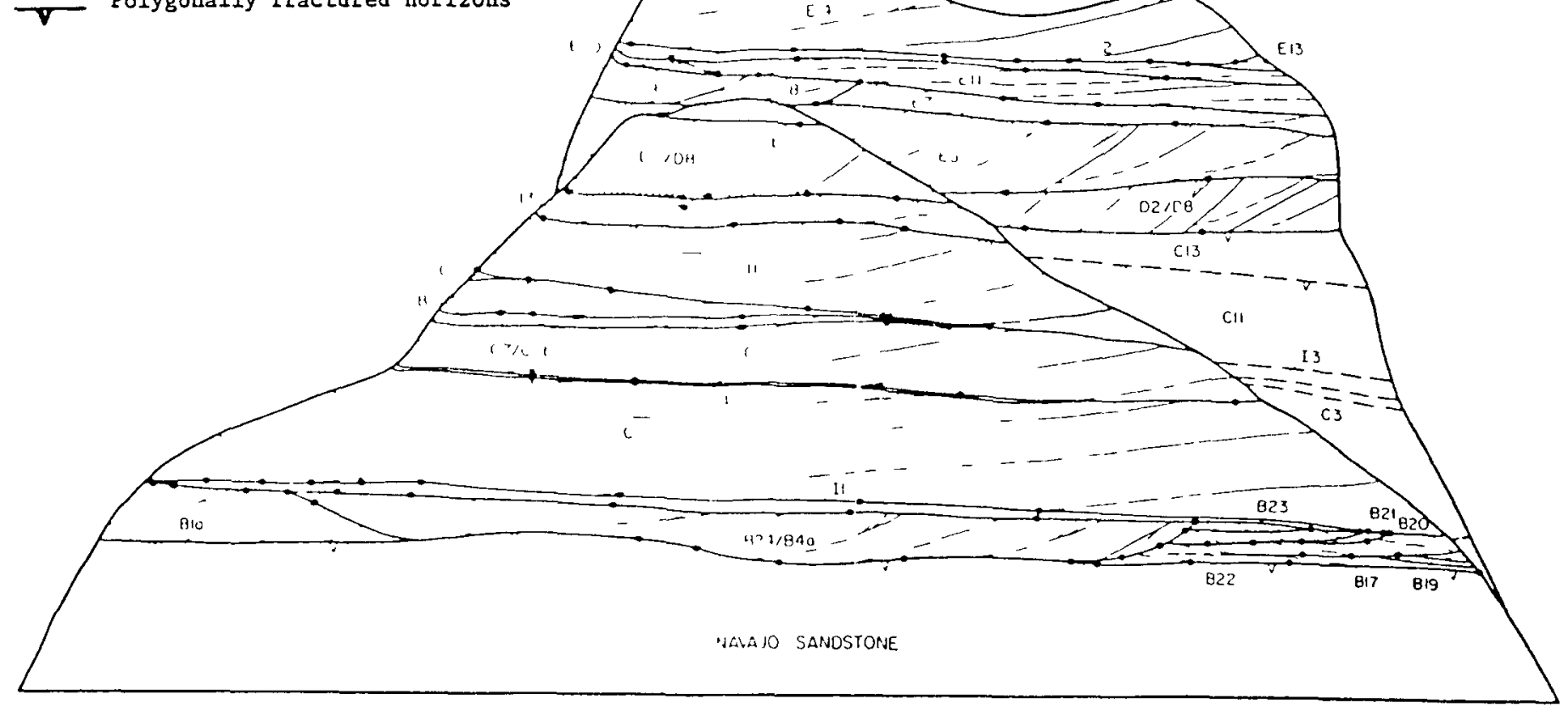

Figure IV-16. Reconstruction of the southeast side of the study knob. 
NORTHEAST WALL (N32W)

EXPLANATION

- Data points

First- and second-order bounding surfaces

Reactivation surfaces

- Grainflow stratification

- - Wind-ripple stratification

P Polygonally fractured horizons

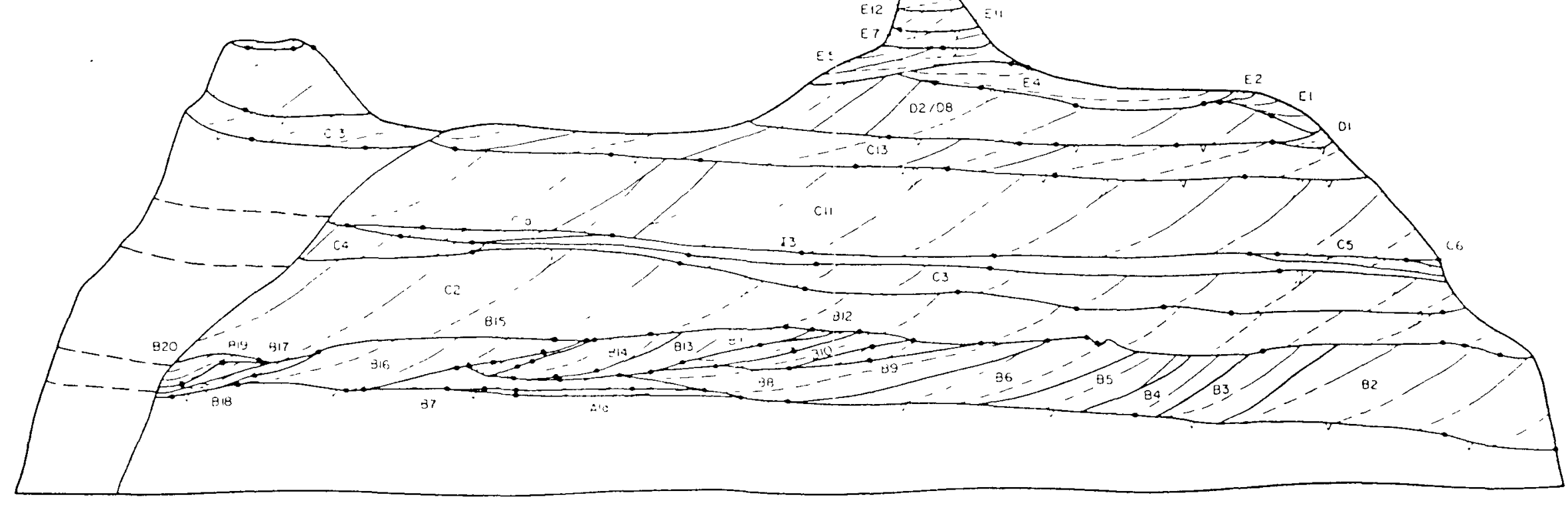

Figure IV-17. Reconstruction of the northeast side of the study knob.

54 tests (Tables IV-1 through IV-5). 
the dipping bounding surface around topographic spurs or in coves, or near the top of the knob where there is a prominent flat-lying ledge (Fig. IV-14).

To determine bounding surface orientations, trend surface analysis was appized to the normalized data set. First-order trend surface analysis fits a planar surface to a given set of measurements and reports the residual, or deviation of the calculated from the measured values (Chorley and Haggett, 1965). For each case, the level of significance was determined to assess whether the planar surface accurately described the bounding surface; at least $95 \%$ of the variation was explained in 39 out of 54 tests (Tables IV-1 through IV-5). From the equations defining each plane, the strike and dip of the bounding surfaces were calculated (See Appendix). To correct for the post-depositional dip component, each surface was rotated about a plane dipping 1.3 degrees N9W. This plane equals the average orientation of 3 of the 4 horizons within the Page Sandstone that locally exhibit polygonal fractures (Fig. IV-11); data for the uppermost, polygonally-fractured horizon do not significantly define a planar surface. These 3 reference horizons are very nearly parallel and are interpreted to represent flat-lying 'Stokes-type' bounding surfaces, formed by water table-controlled deflation (Stokes, 1968). kotating the reference surfaces about the calculated mean yields a total variation in the dip angle of 0.16 degrees. Any surface calculated to dip at an angle less than or equal to this value is therefore statistically lie flat.

Where the size of the data set for a bounding surface is sufficiently large, data points were also fit to a second-order 
(quadratic) surface. The calculated levels of signjficance were compared to the first-order results; 21 of the 32 bounding surfaces were significantly better described by quadratic surfaces than by planar surfaces, indicating a locally ronplanar geometry (see Davis, 1973 for a description of the procedure). However, the accuracy of the strike and dip calculations is inferred from the significance of the first-order results.

In most cases, the average stratification dip direction reported in Tables IV-1 through IV-5 is the calculated mean for each unit. The degree of foreset dispersion is indicated by the level of significance, which is based on the standard deviation. Stereonet plots on a unit-by-unit basis also convey a sense of the degree of directional spread (Figs. IV-18a, b). Where a bimodal distribution of cross-bed data suggests a nonlinear dune shape, the average dip direction of each mode is calculated separately. The reported orientation is the mean of these two averages. In cases where the stratification dip direction rotates along the length of a unit, from gently dipping wind-ripple laminae to gradually steepening grainflow deposits, the orientation of the most steeply dipping foresets better approximates flow conditions, and so is reported instead of the mean (Reiche, 1938; Tables IV-1 through IV-5).

In conjunction with eolian reservoir and fluid-flow modelling studies, the study knob was drilled, logged, and cored (Goggin et al, 1986; Chandler and Kocurek, 1986). Many of the bounding surfaces and stratified sets seen in the core can be correlated with the mapped units (Fig. IV-19), although a certain degree of heterogeneity and measurement error is apparent. Lacking the resolution of the main 


\begin{tabular}{|c|c|c|c|c|c|c|c|c|c|c|}
\hline UenIt & $\begin{array}{l}\text { Botridnas } \\
\text { sample slze }\end{array}$ & $\begin{array}{l}\text { JRFACES ( } \\
\text { Level of } \\
\text { islorder }\end{array}$ & $\begin{array}{l}\text { ) - TREND SI } \\
\text { gniflcance } \\
\text { 2nd or der }\end{array}$ & $\begin{array}{l}\text { IRF ACl AHAL } \\
\text { Dip direc } \\
\text { beforo rot. }\end{array}$ & $\begin{array}{l}\text { sis } \\
\text { lon, dip } \\
\text { aller rol. }\end{array}$ & Sample slze & $\begin{array}{l}\text { CROSS STRATIIFICA } \\
\text { Lovel of Significanco }\end{array}$ & $\begin{array}{l}\text { JoH }(X B) \\
\text { llgan dip dir ecllon }\end{array}$ & Data & 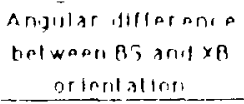 \\
\hline Al & & & & & & 15 & 4758 & & 04 & \\
\hline A2 & & & & & & 10 & 99.58 & 306 & 05 & \\
\hline & & & & & & 6 & 99.58 & 138 & & \\
\hline 43 & & & & & & 2 & $35 x$ & 322 & 1324 & \\
\hline A4 & 11 & $475 x$ & $75 x$ & 356.0 .2 & 176.1 .1 & 8 & 99.58 & 276 & & $(100)$ \\
\hline & & & & & & 6 & 99.58 & 177 & $2 \cdot 8$ & (1) \\
\hline & & & & & & 6 & $90 x$ & 81 & & (103) \\
\hline A5 & 7 & $97.5 x$ & & $357,1.9$ & $357,0.7$ & 13 & $<758$ & & 111 & \\
\hline$A 6$ & 21 & $99.5 x$ & & $301,2.3$ & $301,1.5$ & 18 & $99 x$ & 250 & 15 & 51 \\
\hline A7 & 15 & $99.5 x$ & & $277,2.3$ & $277,2.0$ & 19 & 99.58 & 278 & 210 & 1 \\
\hline$A B$ & 6 & 758 & $97.5 x$ & & & 10 & $995 x$ & 182 & 725 & \\
\hline$\Delta 9$ & 17 & 99.58 & & $46,0.7$ & Flat & 20 & $93 x$ & 161 & 110 & \\
\hline$\wedge 10$ & 14 & 99.51 & & $88,2.3$ & $88,2.2$ & 19 & $93.5 x$ & 113 & 24 & $2 "$ \\
\hline AlI & 15 & 99.58 & & $89,2.6$ & $89,2.5$ & 11 & 93.58 & 140 & 618 & 51 \\
\hline Ala & $\ldots$ & $-\cdots$ & & 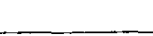 & $\ldots$ & 3 & $93.5 x$ & 202 & 25 & \\
\hline
\end{tabular}

Table IV-1. Bounding surface and stratification data for units of the A-complex. In last column, these values in parentheses are calculated using insufficient data. Where a bi- or trimodal distribution of stratification dip directions is indicated (A4), the mean dip direction of each mode was calculated separately. 


\begin{tabular}{|c|c|c|c|c|c|c|c|c|c|c|}
\hline \multirow[t]{2}{*}{ init } & \multirow{2}{*}{\multicolumn{4}{|c|}{ 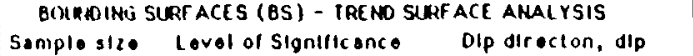 }} & \multirow[b]{2}{*}{ Somplo 120} & \multicolumn{3}{|c|}{ CROSS-STRATIFICAIIOH $(\times B)$} & \multicolumn{2}{|c|}{ Calculaledirendfingulad dilt bet } \\
\hline & & & & & & Level of Shignificence & llesn dip dreclion & DIp range & 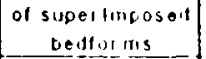 & 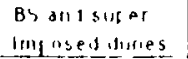 \\
\hline B1 & 8 & 99.51 & $58,5.2$ & $50,4.7$ & 3 & $995 x$ & 157 & 25 & $(i,-1$ & $(\pi 118)$ \\
\hline B2 & & & & & - & 9958 & 111 & $11-24$ & & \\
\hline B3 & & & & & 2 & 998 & 135 & 25 & & \\
\hline B4 & & & & & 1 & 99.58 & 117 & $12-24$ & & \\
\hline BS & & & & & 5 & $99.5 x$ & 125 & 11.18 & & \\
\hline B6 & & & & & 4 & $99.5 \pi$ & 123 & $12-14$ & & \\
\hline B7 & 5 & 958 & $342,0.8$ & $102,0.5$ & 6 & $995 x$ & 145 & $6-16$ & $(61)$ & $(15)$ \\
\hline B8 & 5 & 6758 & $79,23.5$ & $29,23.5$ & 3 & 99.58 & 105 & $3-8$ & (164) & (5) \\
\hline 89 & & & & & 3 & 998 & 127 & $17-26$ & & \\
\hline B 10 & 1 & 75x & $78,35.3$ & $70,35.2$ & 3 & $99.3 \pi$ & 107 & 818 & $(150)$ & $(18)$ \\
\hline 811 & 4 & 952 & $224,19.9$ & $224,19.1$ & 8 & maximum value used & (122) - curved & $4-15$ & 166 & 148 \\
\hline B12 & & & & & 4 & ; $\quad 97.5 x$ & 183 & - 16 & & \\
\hline 813 & 7 & $99.5 x$ & $217,27.2$ & 217,263 & 6 & 9958 & 183 & $6-26$ & Drimaryslipaco & \\
\hline B14 & 4 & ، 258 & $38,7.2$ & $38,0.1$ & 6 & 99.58 & 191 & $11-27$ & $((216))$ & $\left(\left(y_{c}\right)\right)$ \\
\hline B15 & 0 & $99.5 x$ & $48,19.0$ & $40,10.3$ & 5 & "meximum volue used & $(188)$ & 524 & $((232))$ & $((n t))$ \\
\hline ค16 & 0 & 9958 & $105,5.9$ & $105,5.4$ & 13 & meximum value us od & $(180)$ - curvod & 427 & $(103)$ & $(A B)$ \\
\hline 817 & & & & & 4 & 9938 & 142 curvod & 725 & & \\
\hline & & & & & 3 & 9958 & & & & \\
\hline 818 & & & & & 2 & & 38 & 117 & & \\
\hline 819 & & & & & 2 & $95 x$ & 141 curved & 1214 & & \\
\hline & & & & & 3 & 9958 & & & & \\
\hline$B 20$ & 4 & $979 x$ & $112,16.2$ & $112,13.7$ & 9 & $995 x$ & 126 curved & 913 & $(182)$ & $(\cdot(1))$ \\
\hline B21 & & & & & 1 & 9952 & 113 & 627 & & \\
\hline 822 & 1 & 99.58 & $119,12.4$ & 119,119 & 7 & 9958 & 80 & 921 & (122) & $(81)$ \\
\hline B23 & & & & & 4 & $90 x$ & 68 & 2420 & & \\
\hline 824 y & & & & & 7 & 9758 & 100 curvod & 128 & & \\
\hline & & & & & 11 & $995 x$ & & & & \\
\hline Bia & & & & & 14 & 9958 & 112 & 1826 & |urlenarvallfrace & \\
\hline 628 & & & & & 1 & & 108 & 0 & & \\
\hline B3. & 10 & $99.5 x$ & $216,15.1$ & 216.143 & 5 & - & $\underline{2} ! !$ & 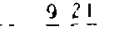 & $(1 / 4)$ & (95) \\
\hline
\end{tabular}

Table IV-2. Bounding surface and stratification data for units of the B-complex. The calculated trends of supertmposed bedforms (second column from the right) were determined using methods suggested by Rubin and Hunter (1983). Those values in a double set of parentheses used a bounding surface dip direction that deviates 180 degrees from the bounding surface dip direction calculated by trend surface analysis. 


\begin{tabular}{|c|c|c|c|c|c|c|c|c|c|c|c|}
\hline \multirow[t]{2}{*}{ Itmit } & \multicolumn{5}{|c|}{ BOINOIHG SURFACE S (BS) - TREND SURF ACE ANALYYSIS (ISA) } & \multirow[b]{2}{*}{ Samplosizo } & \multicolumn{3}{|c|}{ CROSS-STRATIFICATION $(\times 8)$} & \multirow[b]{2}{*}{ Dip range } & \multirow{2}{*}{ 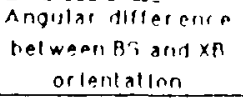 } \\
\hline & Sample sizo & $\begin{array}{l}\text { Level ol Slg } \\
\text { Ist order }\end{array}$ & $\begin{array}{l}\text { nilleance } \\
\text { 2nd or der }\end{array}$ & $\begin{array}{l}\text { Dlp atrect } \\
\text { Defore rot. }\end{array}$ & $\begin{array}{l}\text { lon, alp } \\
\text { aller rot. }\end{array}$ & & & evel of Signifle ance & lloan dip direcllon & & \\
\hline $\begin{array}{l}11 \\
\mathrm{Cl}\end{array}$ & 13 & 99.58 & & $88,0.1$ & $272,1.2$ & & & & & & \\
\hline C2 & 44 & 99.58 & & $77,0.8$ & $77,0.8$ & 18 & & $99.5 x$ & 136 & $6-26$ & s3 \\
\hline C3 & 10 & $99.5 x$ & & $14,2.5$ & $14,1.3$ & 7 & & $99.5 x$ & 148 & $5-24$ & 134 \\
\hline 12 & 9 & $99.5 x$ & & $303,1.9$ & $303,1.0$ & & & & & & \\
\hline C4 & 12 & $(75 x$ & $99.5 x$ & $0,<0.1$ & $180,1.2$ & o & & $99.5 x$ & 142 & $19-25$ & (38) \\
\hline 13 & $\theta$ & $99.5 x$ & & $340,1.3$ & Flol & & & & & & \\
\hline C5 & & & & & & 2 & & $95 x$ & 153 & $17-25$ & \\
\hline Cls & & & & & & 1 & & & 132 & 17 & \\
\hline C6 & & & & & & 1 & & & 252 & $3 ?$ & \\
\hline $\mathrm{Clb}$ & 3 & $97.5 x$ & & $314,3.0$ & $314,2.8$ & 4 & 1 & 99.58 & 165 & $8 \cdot 22$ & 149 \\
\hline$c 7-2 b$ & 14 & $99.5 x$ & & $342,1.0$ & $162,0.4$ & 12 & & $99.5 x$ & 148 & $2-21$ & 11 \\
\hline C3b & 6 & $97.5 x$ & & $289,3.5$ & $289,2.9$ & 11 & & $99.5 x$ & 180 & 327 & 1109 \\
\hline$C 8$ & 14 & $99.5 x$ & & $153,1.1$ & flat & 9 & : & 99.51 & 152 & 023 & \\
\hline$c 9$ & 9 & $99.5 x$ & & $90,1.4$ & Flat & 8 & 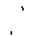 & $99.5 x$ & 178 & $2-25$ & \\
\hline $\mathrm{ClO}$ & 6 & $99.5 x$ & & $206,2.9$ & $206,1.9$ & 6 & & $99.5 x$ & 174 & $5-26$ & 32 \\
\hline Cll & 31 & $99.5 x$ & & $42,2.0$ & $42,1.2$ & 27 & & $99.5 x$ & 148 & $1-27$ & $10 \%$ \\
\hline \multirow[t]{2}{*}{$\mathrm{C} 12$} & 7 & $90 x$ & $75 x$ & trough oxts & calculated & 7 & & $99.5 x$ & 193 & $18-30$ & (5) \\
\hline & & & & by ISA & 198 & 4 & & 99.58 & & & \\
\hline C13 & 31 & $99.5 x$ & & $355,1.4$ & Flal by dol. & 48 & & $99.5 x$ & 185 & $5-18$ & .. \\
\hline
\end{tabular}

Table IV-3. Bounding surface and stratification data for units of the C-complex. In the last column, these values in parentheses are calculated using insufficient data. 


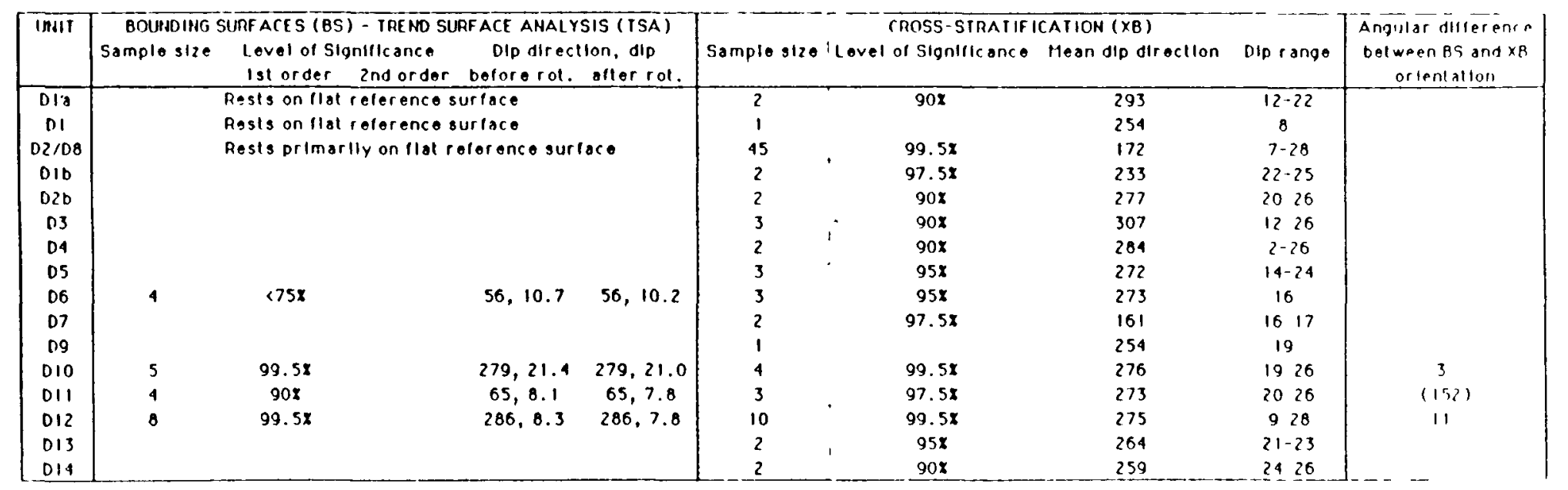

Table IV-4. Bounding surface and stratification data for units of the D-complex. In last the column, these values in parentheses are calculated using insufficient data. 


\begin{tabular}{|c|c|c|c|c|c|c|c|c|c|c|}
\hline \multirow[t]{3}{*}{ UtwI } & \multicolumn{5}{|c|}{ BOUHD INU SURFACES (BS) - TREND SURFACE ANALYSIS (TSA) } & \multicolumn{4}{|c|}{ CROSS STRATIFICATIOH (XB) } & \multirow{3}{*}{$\begin{array}{l}\text { Angular differeme } \\
\text { bolwoon ba arme ab } \\
\text { orlerlation }\end{array}$} \\
\hline & Saniplesizo & Level of & unific ance & Dip diroct & Ion, dip & Samplosizo & Lovel or signillicanco & llodn dip direcllon & Dipranyo & \\
\hline & & lol ordar & 2nd order & Ealoreral. & aller rot. & & & & & \\
\hline [1 & \multicolumn{5}{|c|}{ Rosts prinarlly on flat reforence surfaco } & 10 & $99.3 x$ & $150-$ curved & $11-30$ & \\
\hline & & & & & & 8 & $99.3 x$ & & & \\
\hline E2 & & & & & & 4 & $99 x$ & 105 & 1319 & \\
\hline E 3 & & & & & & 3 & $90 x$ & 230 & $9-23$ & \\
\hline Cla & & & & & & 10 & $99.5 x$ & 72 - curvod & $11-14$ & \\
\hline E20 & & & & & & 1 & & 64 & 14 & \\
\hline EA & 7 & $<75 x$ & $90 x$ & $357,3.5$ & $357,2.3$ & 16 & maximurn value used & $140-$ curved & $4-28$ & $(1+3)$ \\
\hline E5 & 10 & $90 x$ & $99.5 x$ & $\begin{array}{l}\text { Irough axis } \\
\text { by TSA }\end{array}$ & $\begin{array}{c}\text { calculatod } \\
175\end{array}$ & 11 & $\begin{array}{c}\text { Irough dxis measured } \\
\text { in fleld }\end{array}$ & 145 & $12-25$ & $(30)$ \\
\hline E6 & 11 & $99.5 x$ & & $314,5.5$ & $314,4.5$ & 14 & $99 x$ & 147 - curved & $6-26$ & 167 \\
\hline E7 & \multicolumn{5}{|c|}{ Rests on flat reference surface } & 12 & 99.58 & 195 & $2-23$ & \\
\hline E 8 & \multicolumn{5}{|c|}{ Rosts primarliy on flat reforence surlace } & 9 & $99.5 x$ & 202 - curvos & 619 & \\
\hline E9 & \multicolumn{5}{|c|}{ Rests primarlly on flat reforence surlaco } & 7 & $99.5 x$ & 201 & $5-22$ & \\
\hline E 10 & 6 & <75x & $<75 x$ & $66,0.5$ & $66,0.2$ & 6 & $99.5 \pi$ & 157 & $4-14$ & $(91)$ \\
\hline E11 & \multicolumn{5}{|c|}{ Rosts parllally on polygonal time line } & 8 & $99.5 x$ & 126 & $3-25$ & \\
\hline E 12 & 18 & $99.5 x$ & & $319,1.9$ & $319,0.8$ & 8 & $99.5 x$ & 131 & 25 & $1 / 2$ \\
\hline$[13$ & 9 & $، 75 x$ & $<75 x$ & $284,0.5$ & Fial & 7 & $99.5 x$ & 131 & $6 \quad 17$ & \\
\hline E19 & 16 & $73 x$ & $95 x$ & $116,1.6$ & $116,0.9$ & 8 & 99.32 & $1+4$ & 2120 & $(28)$ \\
\hline E 10 & & & & & & 2 & 998 & $1 / 5$ & 815 & \\
\hline$\underline{E} \underline{2} \underline{0}$ & & & & & & 2 & $\underline{90 x}$ & 100 & $1 \pm 18$ & \\
\hline
\end{tabular}

Table IV-5. Bounding surface and stratification data for units of the E-complex. In the last column, these values in parentheses are values calculated using insufficient data. 
A 1

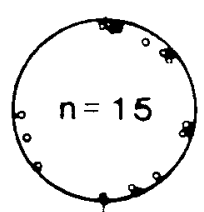

A 7

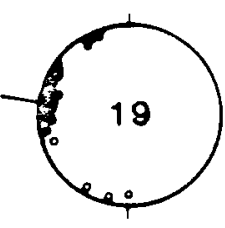

A2

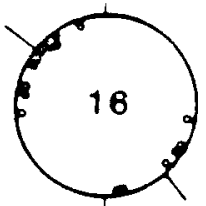

A 8

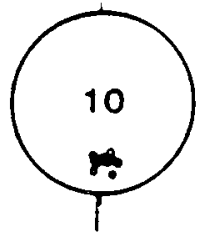

A 4

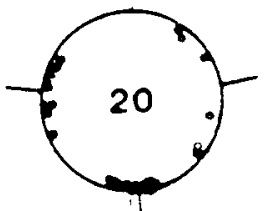

A 9
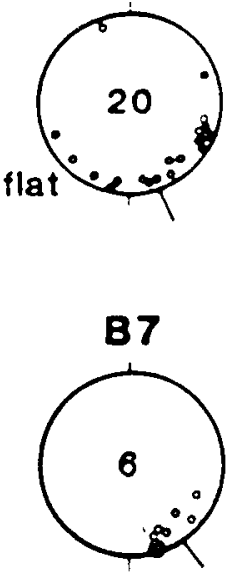

B16
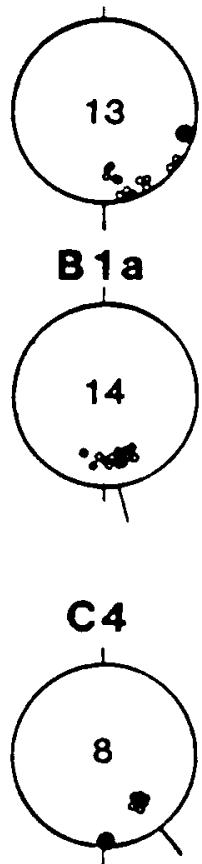

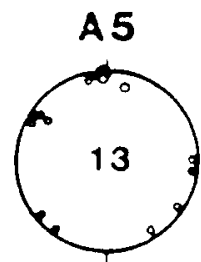

A 10

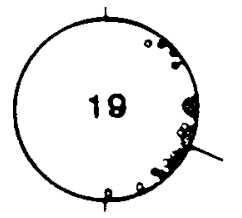

B11

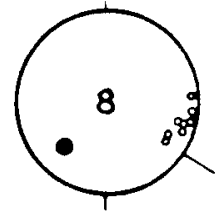

B 17-B19
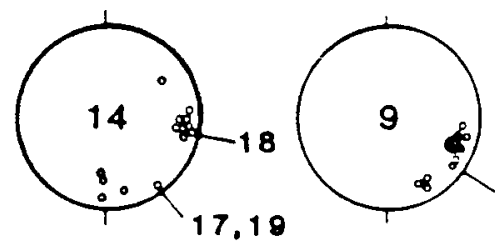

B3a

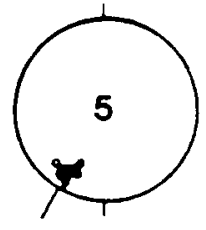

C7-2b
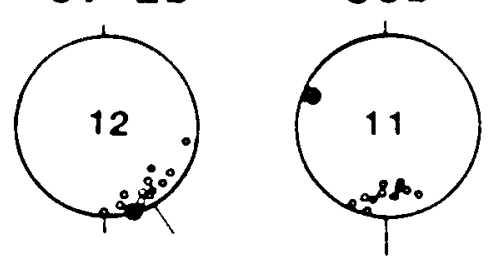

Figures IV-18a. Stereonet plots of stratification and bounding surface dip directions for units of the study area. 

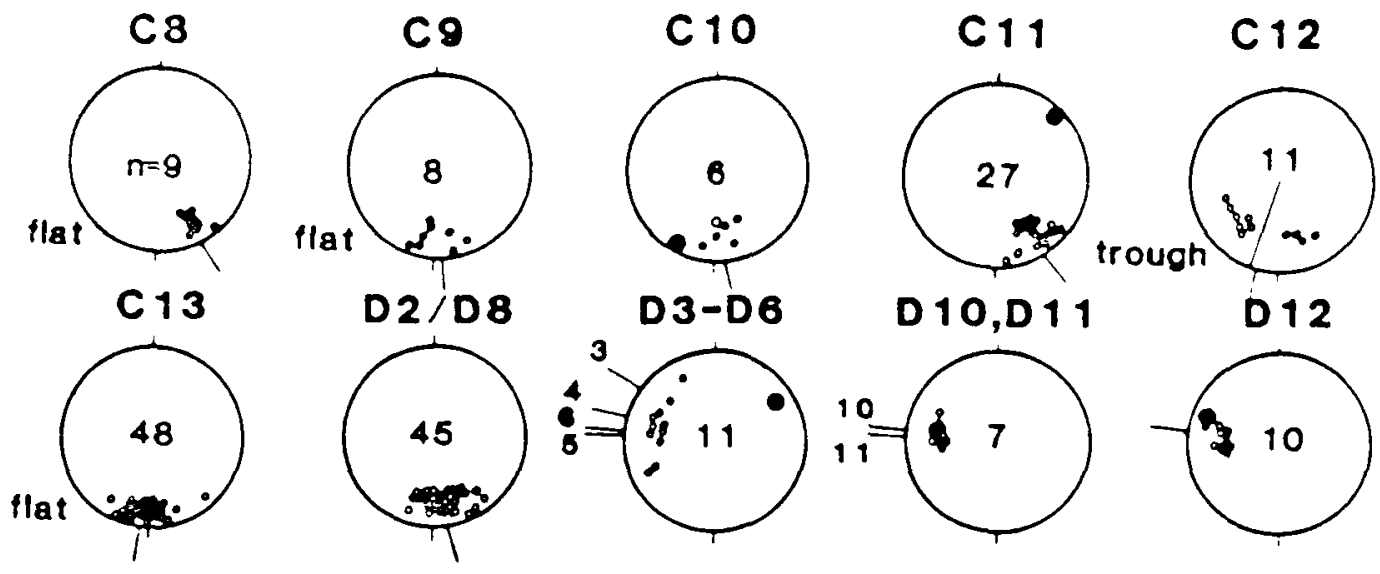

D 12
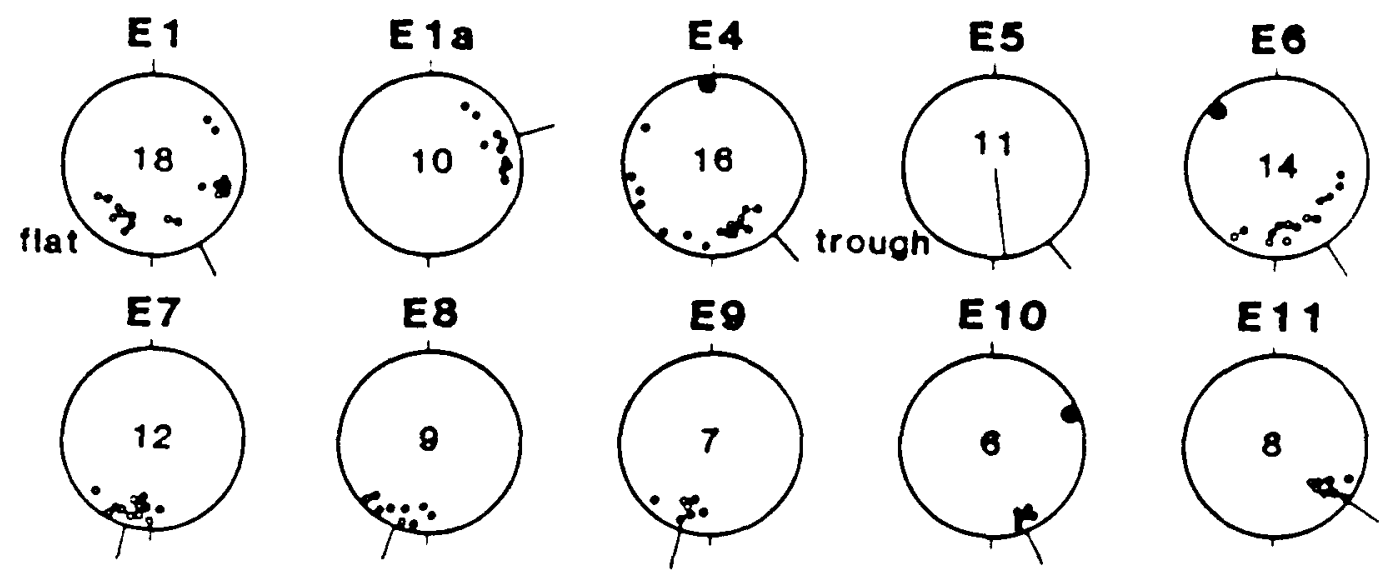

E11

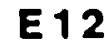

E13

E14
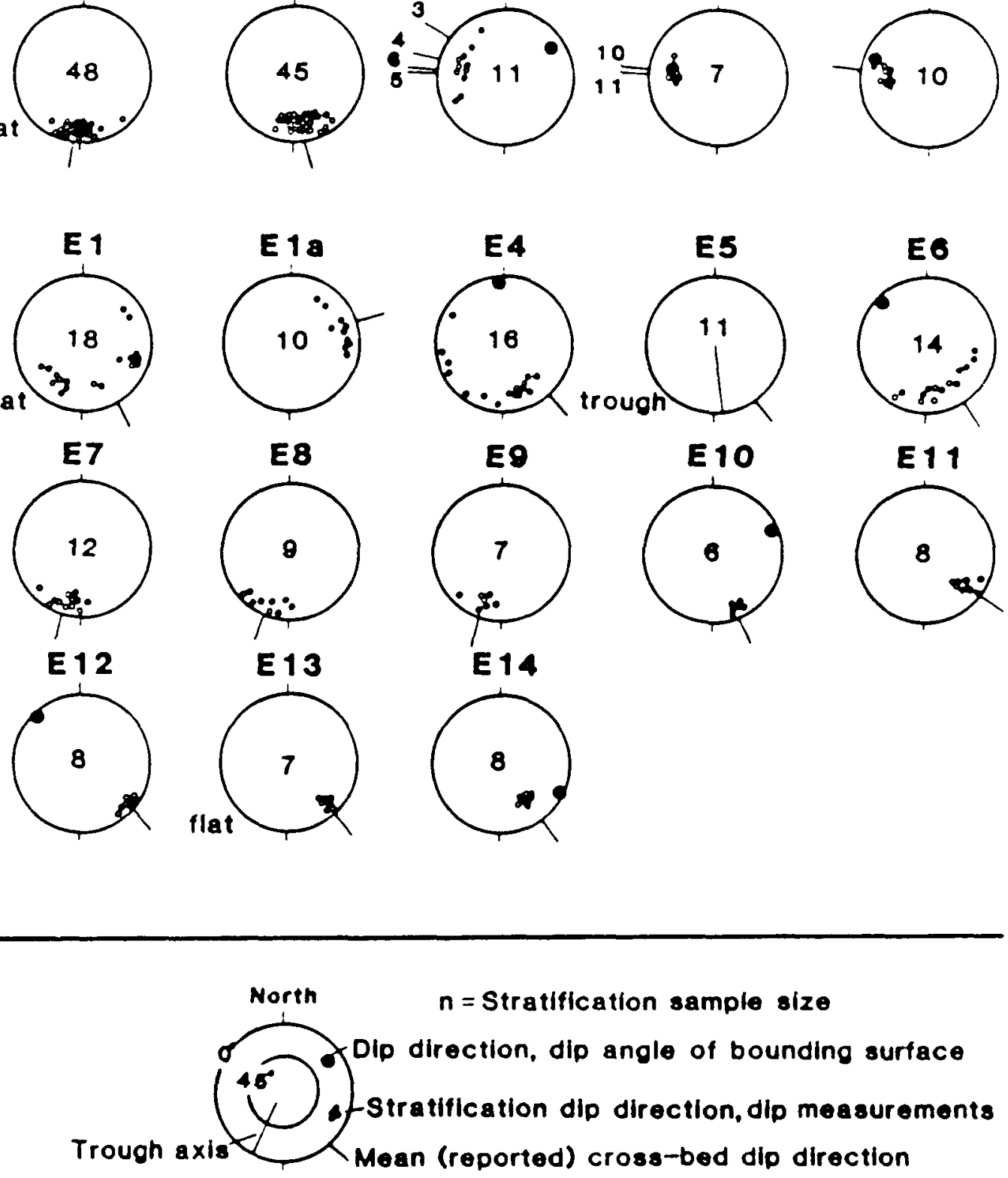

Figures IV-18b. Stereonet plots of stratification and bounding surface dip directions for units of the study area. 


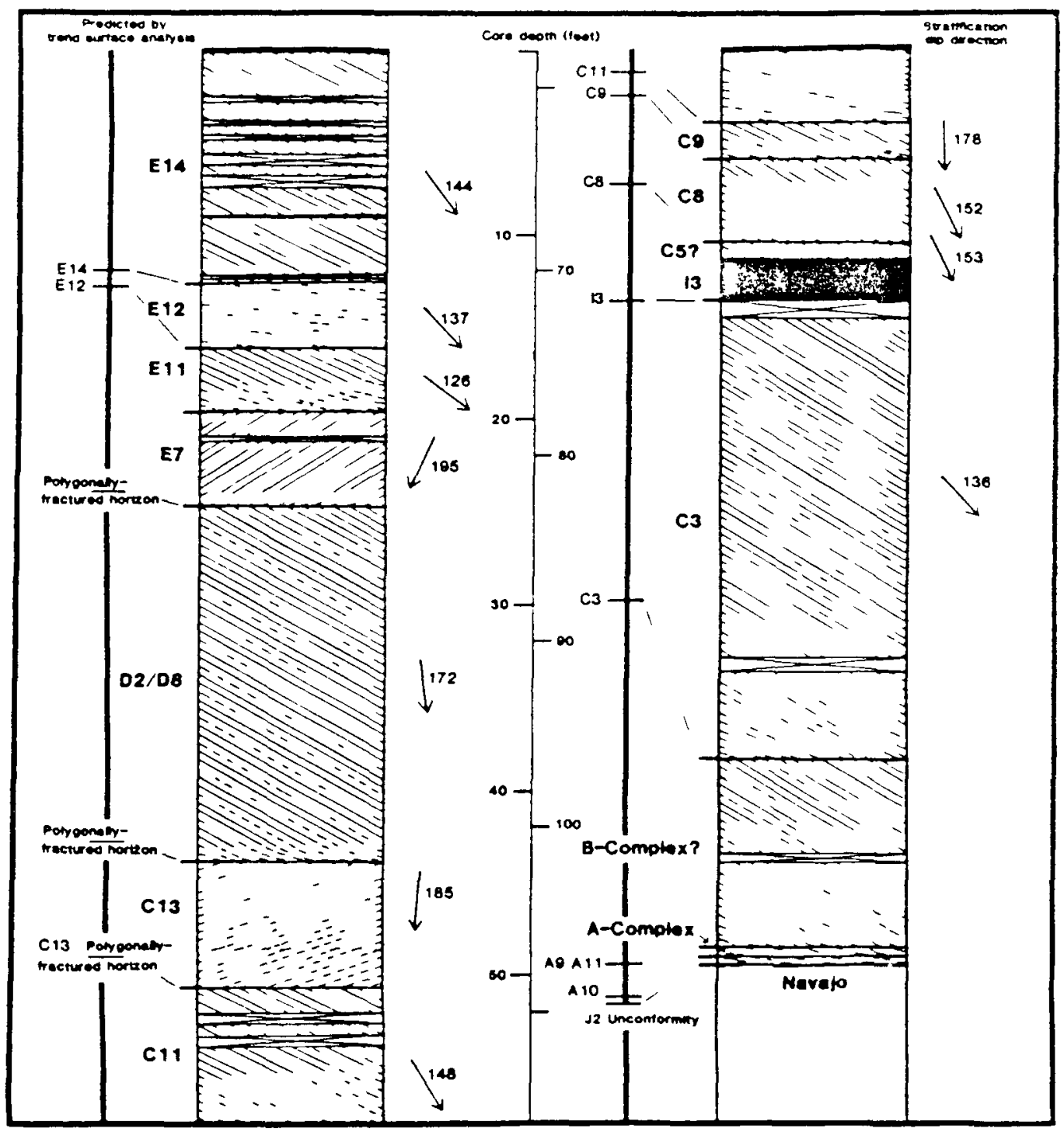

Figure IV-19. Description of the core retrieved from the study site, January 1986. For comparison with the results of trend surface analysis, the depth reading on the core and of the regional bounding surface associated with the extra-erg deposits of the Carmel Formation (I3) was set equal to the depth as predicted by trend surface analysis. 
data set, depth measurements from the core could not be used directly in trend surface analysis. Core data did, however, support the findings of the study.

The Study Knob Reconstructed - A total of 92 depositional units were identified at the study site. Details of their stratification, and of the bounding surfaces separating them are summarized in Figs. IV-14 through IV-17 and in Tables IV-1 through IV-5. The entire sequence can be divided into five subfacies or complexes. Differences in depositional style distinguish the subfacies, which form the framework for the final interpretation. Units are prefixed with the letter corresponding to the complex to which they belong. Where stratigraphic relationships cannot be resolved, a subset of one or more units identified with lower case letters is established (for example, Unit Ala forms a part of the A-complex, but it cannot be correlated with other units of the same complex; Figs. IV-14, IV-17).

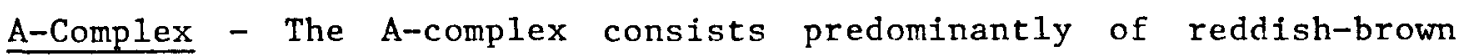
wind-ripple strata deposited directly on the J? unconformity (Figs. IV-14, IV-20). Twelve of the 13 units of the complex are confined to a local depression in the J2 surface, where polygonal fractures are notably conspicuous (Fig. IV-21). They are distinguished from overlying units by their darker color, greater degree of cementation, and their coarser, bimodal texture; maximum grain size approaches $1 \mathrm{~mm}$. A8 holds distinction as the single occurrence of grainflow deposits in this basal sequence (Figs. IV-14, IV-20), where roughly $30 \mathrm{~cm}$. thick 


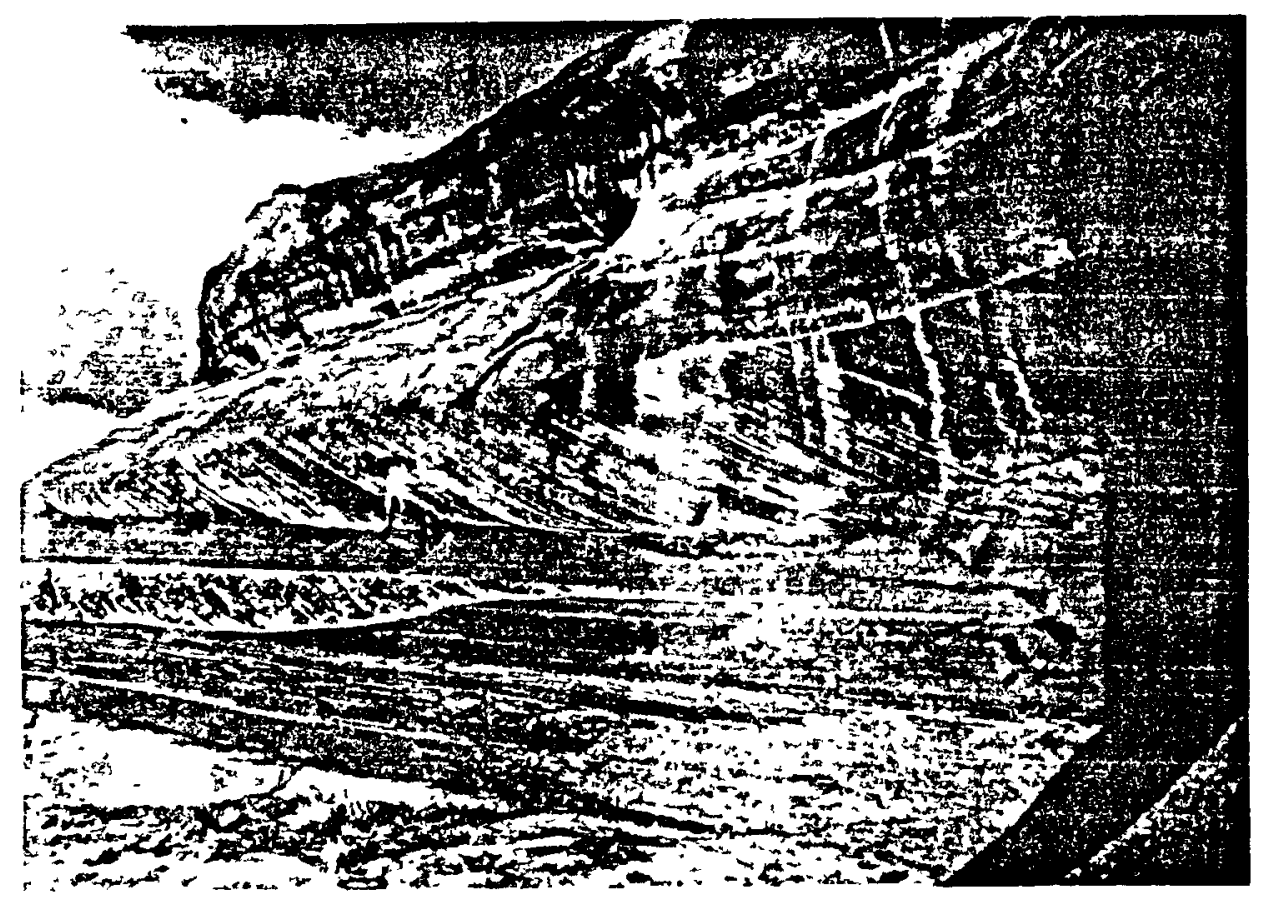

Figure IV-20. The wind-ripple-stratified deposits of the A-complex, northwest wall of the study site. The gray, lensshaped unit is the single local occurrence of grainflow stratification within the A-complex (A8; Table IV-1). Unit All, which caps the A-complex, is the set of relatively steeply-dipping, striped strata in the middle of the photo, and are interpreted to have been deposited by migrating zibars. 


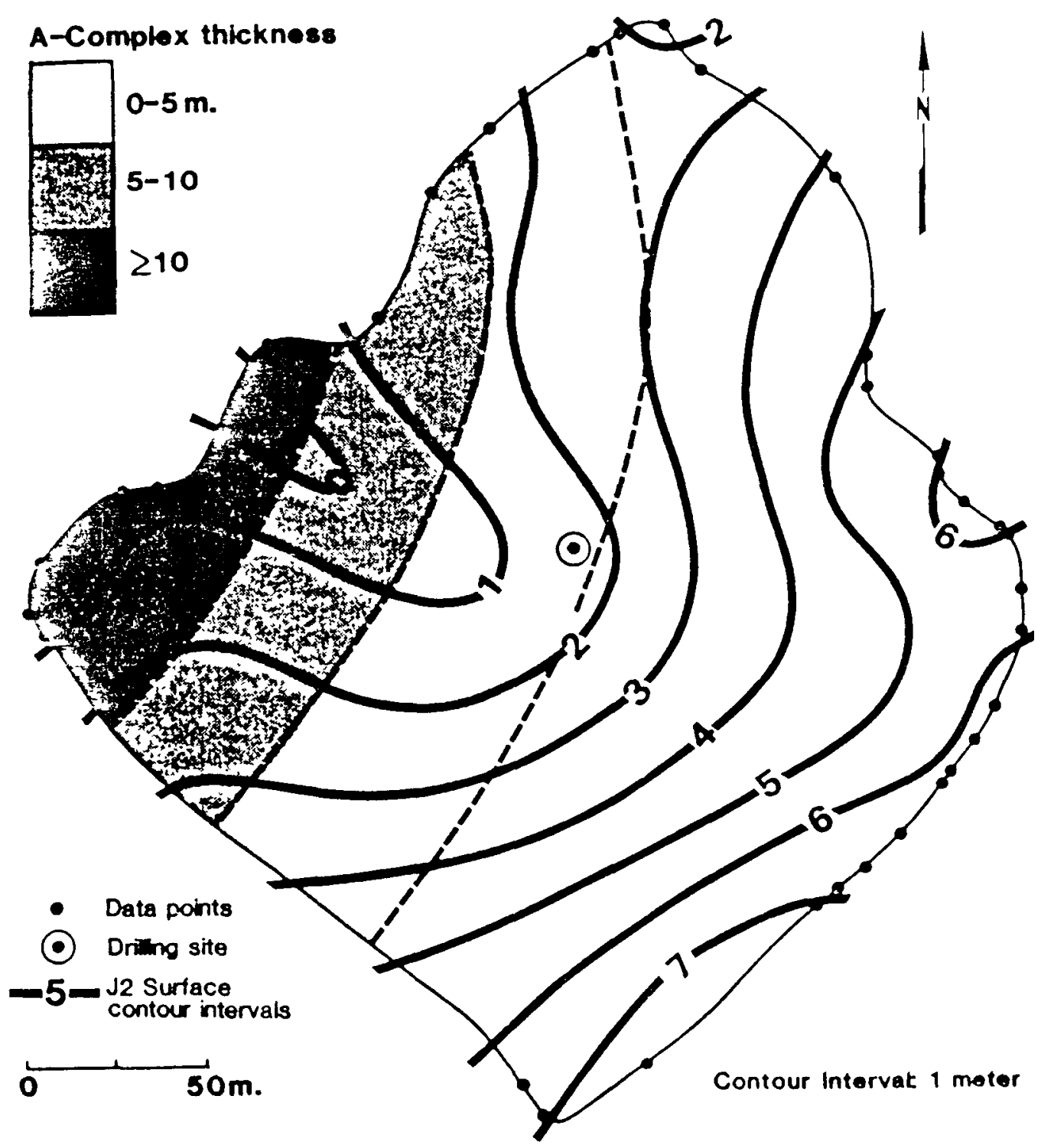

Figure IV-21. Structure contour map of the J2 surface at the study site. The thickness of the overlying A-complex is represented by the shaded bands. Deposits of the A-complex appear to be restricted to a local depression in the $\mathrm{J} 2$ surface. 
couplets of coarser- and finer-grained strata bounded by thin windripple laminae define its cyclic depositional character.

Proceeding upwards through the complex, wind-ripple deposits very generally exhibit an improved sense of directionality and increasingly steeper dip angles. Accompanying this trend, the mean stratification dip direction of successive units sweeps from the southwest to the southeast (Fig. IV-18a, Table IV-1). Within each unit, wind-ripple laminae show a marked parallelism, and dip in the same direction or slightly oblique to the corresponding bounding surfaces.

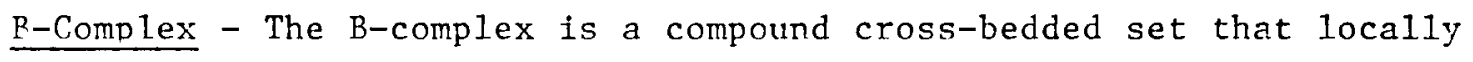
truncates the A-complex and rests on the elevated portion of the J2 surface (Figs. IV-16, IV-17). Within the sequence, three 'episodes' occur, where units dominated by steeply dipping grainflow give way to units composed exclusively of gently dipping wind-ripple stratification (B1 through B8, B9 through B12, and B16 through B20; Table IV-2). Individual grainflow strata are particularly thick- up to $11 \mathrm{~cm}$-- in units $\mathrm{B} 13$ and Bla. Basal apron deposits for both units are relatively thin, although $B l a$ grades laterally into wind-rippled flank deposits. Two scales of cyclicity are apparent in Bla: packages of grainflow strata from 20 to $40 \mathrm{~cm}$. thick are bounded by thin but distinct wind-ripple laminae (Fig. IV-3). Additionally, grainflow deposits showing a greater degree of reworking ('wind-rippled grainflow') define a poorly-developed cyclicity on the order of ten (?) meters.

Deposits of the B-complex dip consistently towards the southeast quadrant (Fig. IV-18a). The distribution of dip directions across 
some of the units suggest curved crestlines, although the evidence is generally very subtle. Lateral truncation of these compound cross-bedded units contributes to the restricted range of foreset dispersion, which is as little as 25 degrees. Both planar and trough-shaped second-order bounding surfaces occur within the B-complex. Of the bounding surfaces significantly described by trend surface analysis, most dip 15 degrees or less (Table IV-2). The second-order surface associated with unit B13 dips most steeply - 26 degrees to the southwest.

C-Complex - The C-complex includes both laterally extensive simple cross-bedded sets and thin, horizontally laminated to massive deposits. Most of the cross-stratified units feature wind-rippled apron deposits at their base, in some cases to the exclusion of any preserved grainflow deposits (for example, Cl0; Fig. IV-15b). The notable exception is unit $\mathrm{C} 4$, where packages or bundles of grainflow laminae roughly $13 \mathrm{~cm}$. thick extend to the base of the unit. Individual grainflow stratum thickness for units of the C-complex ranges from 3 to 6 centimeters. Larger-scale cyclicity defined by intervals of reworked grainflow occurs in some of the units as discontinuous patches. As in units of the subjacent B-complex, strata of the C-complex dip to the south and southeast, although any sense of deposttional episodictty is lacking.

Units I1 and I2 (Fig. IV-15b, IV-16) are composed of indistinct, horizontal wind-ripple laminations that are texturally less mature than the cross-bedded sets (See Chandler and Kocurek (1986), for textural details of the deposits of the study site). These deposits 
overlie first-order bounding surfaces and are thin and broadly lenticular. Unit I3 stands out as a deep red, texturally immature bed approximately 1 meter thick, which can be traced northwestwards to the Judd Hollow tongue of the Carmel Formation.

After correcting for post-depositional subsidence, I3 appears to have been deposited upon a flat bounding surface. Two other horizons within the complex, as well as the polygonally-fractured reference surfaces bounding unit $\mathrm{C} 13$, also lie flat (Table IV-3). With the exception of units $\mathrm{C} 4$ and $\mathrm{C} 12$, al1 bounding surfaces to which trend surface analysis was applied are very accurately described by a planar surface. $\mathrm{C} 4$ is the one unit of the C-complex that lacks apron deposits, and $\mathrm{C} 12$ is a trough-shaped unit near the top of the complex (Figs. IV-14, Iv-15a). Going upwards through the C-complex, bounding surface dip directions show no predictable pattern, much less a consistent orientation, and their relationship to corresponding stratification dip directions varies from highly oblique orientations to nearly parallel (Figs. IV-18a, IV-18b).

D-Complex - Units of the D-complex are highly weathered deposits atop the southwestern portion of the study knob. Their exposure on a prominent ledge makes these deposits appear distorted in cross-section (Fig. IV-14). The planimetric view detailed in Fig. IV-22 is a more realistic representation. Within the sequence, grainflow-stratified units dipping west north-west show a complex stratigraphic relationship with the southward-dipping deposits of D2/D8. Deposition of the D2 component preceded deposition of units D3 through D7. The character and the orientation of the deposits of the D8 component are 


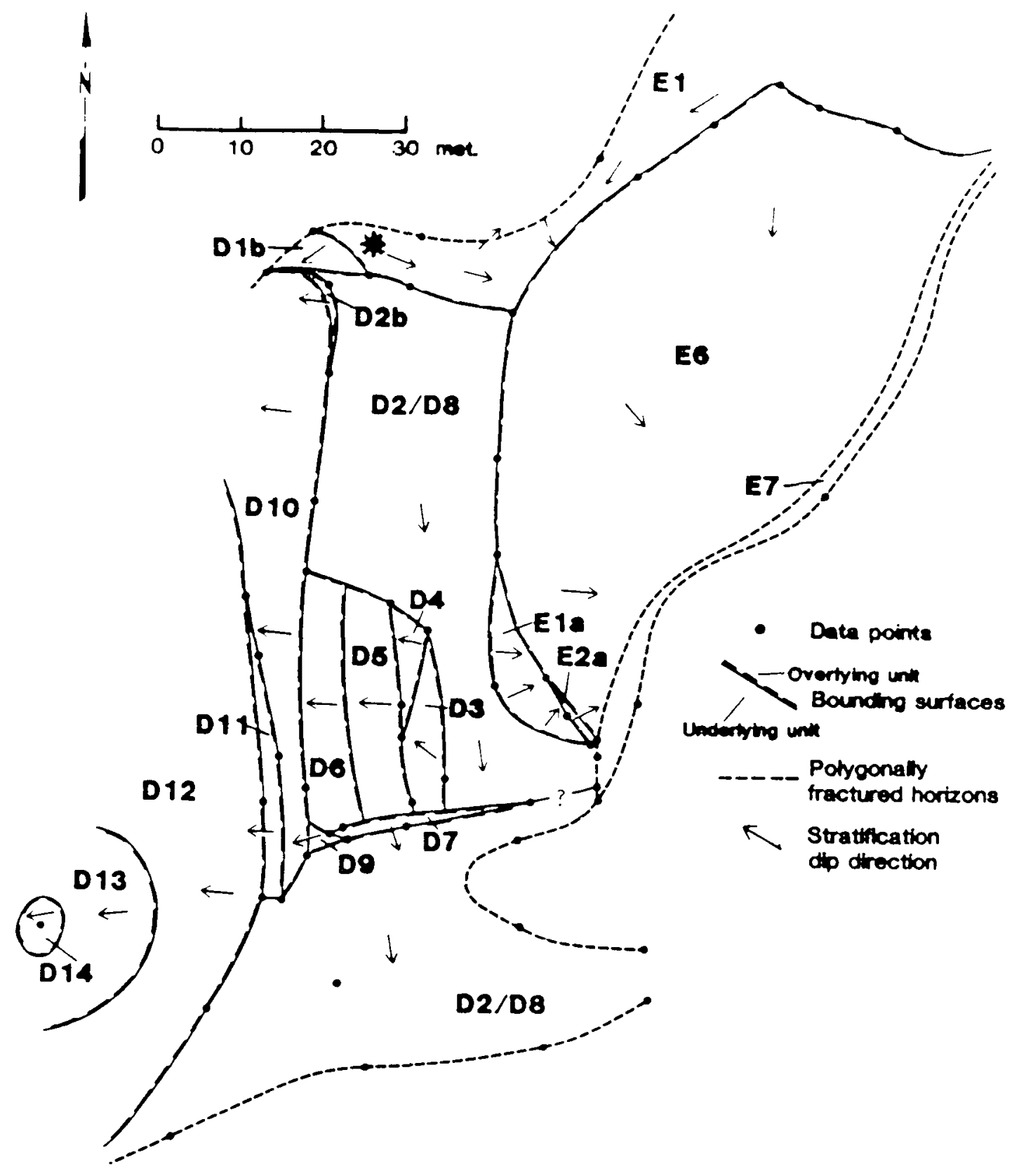

Figure IV-22. Detail of Fig. IV-13, showing a planimetric view of the units of the D- and E-complexes that are exposed on a flat-lying ledge near the top of the study ste. 
identical to those of the D2 component, yet they overlie the southward-dipping sliver D7. It is not an unreasonable contention that $D 2 / D 8$ represents a time-transgressive unit whose downdip migration and deposition was interrupted by local deposition of sands dipping westward. Units D9 through D14 cap the sequence and likewise dip west north-west. D2/D8 consists mostly of individual grainflow strata up to $6 \mathrm{~cm}$ thick, crossed by numerous reactivation surfaces. Low-angle apron deposits occur discontinuously across its length and otherwise constitute a minor proportion of the total set.

D2/D8 strata rest primarily upon the same flat reference surface that truncates the wind-rippled deposits of C13 (Fig. IV-19). Where trend surface analysis yields significant results for the other units of the D-complex, bounding surfaces dip steeply to the northwest, paralle1 to the stratification dip direction (Table IV-4; Fig. IV-18b).

E-Complex - The E-complex includes the uppermost 18 units of the Page Sandstone at the study site (Figs. IV-14 through IV-17). Like the C-complex most of the units of the E-complex consist of grainflow-stratified sets $3-5 \mathrm{~cm}$. thick over relatively thick wind-rippled apron deposits. Units EI and E14, the lowest and highest members of the complex respectively, lack apron deposits and exhibit a poorly developed, larger-scale cyclicity defined by reworked grainflow deposits.

The distribution of foreset dip directions across many of the lower units suggest curved crestlines (Fig. IV-22). Strata dip generally to the southwest or to the southeast and show more inter- 
and intra-unit variability than the comparatively unidirectional deposits of the C-complex (Table IV-5, Fig. 18b). E1 is a particularly complicated unit where compound cross-bedding dippirg to the east and northeast is separated from southward-dipping, simple cross-sets by numerous reactivation surfaces (Figs. IV-22, IV-23). Together, these components define a strongly curved sequence whose mean dip direction to the southeast compares with other units of the E-complex.

Two polygonally fractured surfaces occur within the E-complex, the uppermost not accurately described by trend surface analysis. Like other units resting atop such horizons, E7 contains numerous reactivation surfaces. There is also a correlation between polygonally fractured surfaces and trough-shaped units at the study site; C12 and E4, the two distinctly trough-shaped units found locally within the Page, are both truncated by a polygonally fractured horizon (Figs. IV-15a, IV-b).

Eolian Bedform Reconstruction - (An Application of the Tools to the Deposits of the Study Site)-Armed with observations and data that characterize the deposits of the study knob, the ability of the tools described above to reconstruct and interpret eolian bedforms can be tested. The resolution and the limitations of each tool, as applied to the deposits of the Page Sandstone, reflect in part the present level of understanding of eolian depositional systems and processes.

The origin of bounding surfaces - Regional bounding surfaces within the sequence studied are identified by the overlying extradune deposits of I3 and by polygonal fractures, both of which mark a hiatus 


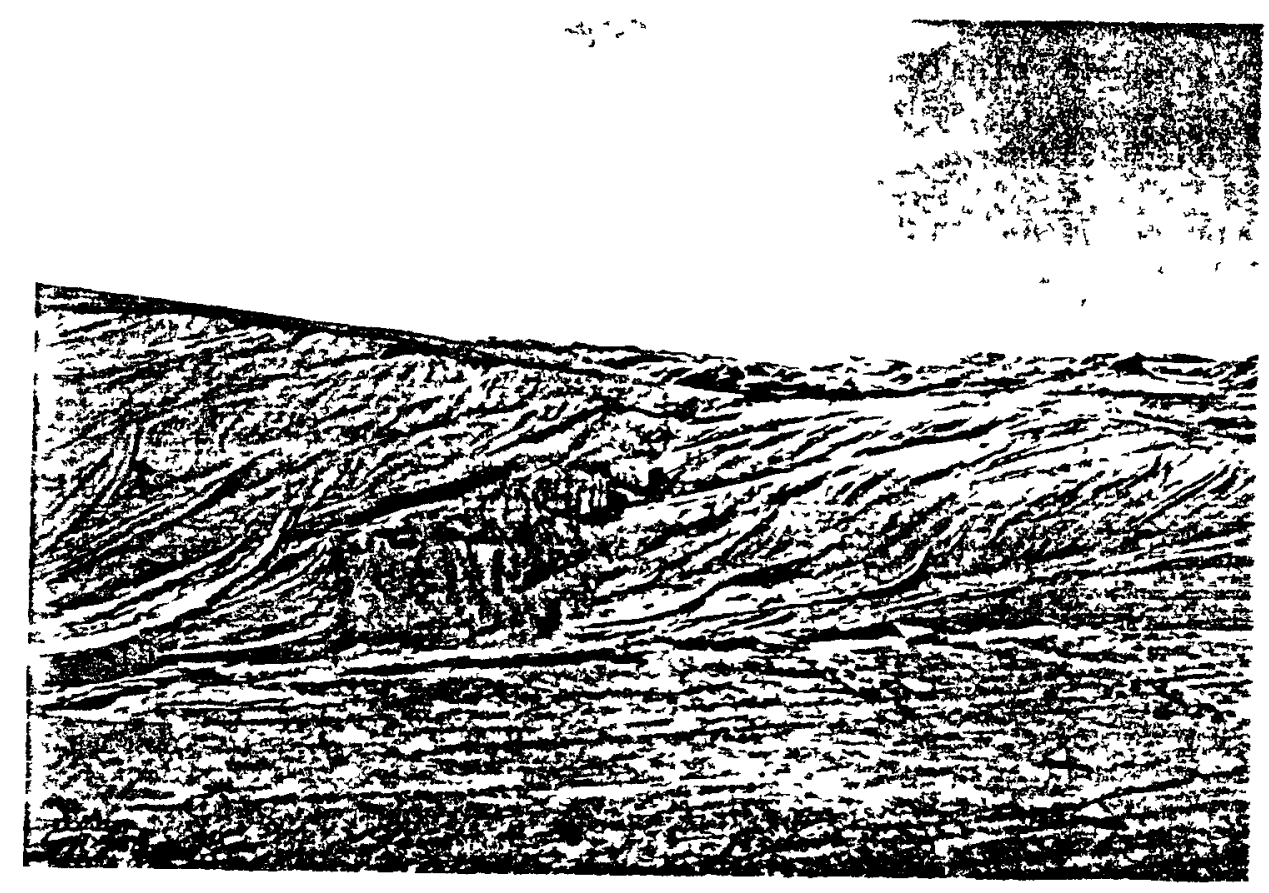

Figure IV-23. The compound cross-bedded deposits of unit E1. Specific location of photo is indicated by a "*" in Fig. IV-22. Reactivation surfaces (left) separate the simple cross-bedded deposits of El (not shown in photo) from steeply dipping compound cross-sets (right). 
in sand sea development (Fig. IV-24). Within the resolution of the data, the lowermost 3 of the 4 polygonally fractured horizons within the Page, and the bounding surface associated with I3 parallel each other, and are assumed to define 'paleo-horizontal'. Local J2 relief overprints the regional trend of the J2 unconformity (Fig. IV-21), and so does not appear to parallel the orientation of the other regional bounding surfaces in the sequence. Other surfaces found locally within the Page exhibit a parallel orientation (those surfaces listed in Tables IV-1 through IV-5 as lying flat after rotating their first-order planes about the orientation defining 'paleo-horizontal'), but otherwise lack any indications of prolonged exposure and changing depositional conditions. The subhorizontal bounding surfaces of the C- and E-complexes are interpreted to represent first-order bounding surfaces on the basis of uniform spacing within the sequence and the presence of thin interdune deposits.

Compound cross-bedding of the $B-$ and D-complexes were deposited by superimposed bedforms migrating across the lee face of a draa (Fig. IV-24). In both cases, the transverse extent of the surfaces appears small, and evidence of variable winds and flow reversal are lacking. Reactivation surfaces within the B-complex, truncated by the second-order surfaces, are relatively sparse. Moreover, compound cross-strata in both complexes grade laterally into simple cross-bedded draa slipface deposits (Bla and B13 for the B-complex, and D2/D8 for the D-complex). Reactivation surfaces are particularly common in those units associated with regional bounding surfaces ( 11 , C13, D2/D8, E7) and lack the cyclicity of compound cross-bedding deposited by superimposed bedforms. 


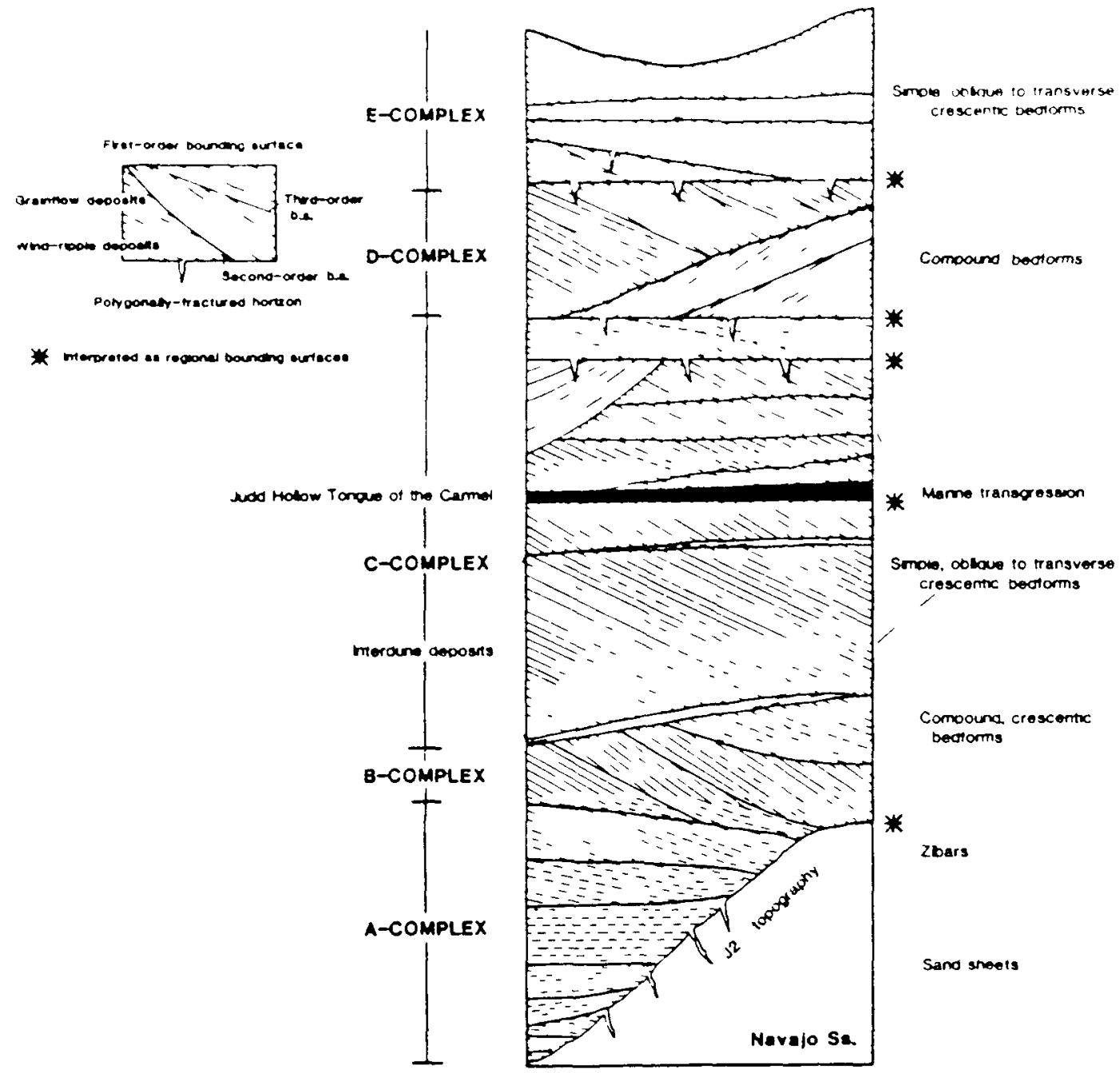

Figure IV-24. Schematic cross-section and interpretation of the cross-bedded sequence of the Page Sandstone at the study site. 
Interpreting Low-angle Stratification - Coarse grain size, evidence of the former presence of evaporites on the polygonally-fractured J2 surface (see Kocurek and Hunter, 1986), and their position at the base of the Page Sandstone in a local depression, favor a sand-sheet interpretation for the wind-ripple-stratified deposits of the A-complex (Fig. IV-24). Sands of the A-complex are too coarse to represent dune apron deposits, and the presence of evaporites would have likewise inhibited dune formation.

The internal parallelism of gently dipping laminae is not characteristic of sand-sheet deposits, but may reflect the control of J2 relief on deposition, which under relatively sand-starved conditions was restricted to depressions in the otherwise deflationary J2 surface. Complex primary and secondary airflow and the deposition of variably oriented wind-ripple strata were succeeded by a more unimodal sand transport pattern as $\mathrm{J} 2$ relief was subdued by infilling. As airflow became less variable and/or net sand supply increased, migrating zibars deposited the unidirectional, moderately-dipping wind-ripple strata of All.

Other low-angle wind-ripple sets in the sequence represent either interdune or dune apron deposits. Interdune deposits in the study area (I1 and I2) tend to be thinner, more poorly sorted (Chandler and Kocurek, 1986), and component laminae more gently dipping than dune apron deposits. Unit $\mathrm{C} 12$ is an example of a locally-occurring dune apron deposit characterized by moderately dipping wind-ripple strata, whose thickness and lateral extent are comparable to other deposits in the sequence left by migrating dunes (Fig. IV-19). 
The degree of crestline curvature - A gradual swing in foreset dip direction along the lengths of many of the units of the B-complex and the E-complex suggest that the bedforms represented were nonlinear. A three-dimensional bedform interpretation is supported by trend surface analysis, which indicates that bounding surfaces within the E-complex are relatively nonplanar. The original bedforms that deposited the C-complex were comparatively linear, although broadly lenticular interdune deposits and larger-scale cyclicity of reworked grainflow deposits suggest some degree of crestline curvature. In fact, most of the units in the study area include at least one nonlinear dune shape indicator. That intervals of reworked grainflow deposits present within a unit result from the shifting morphology of a curved bedform is conjecture at this point; further studies are recommended before their presence is cited as conclusive evidence of crestline curvature.

Dune dimensions - Estimates of the dimensions of the original bedforms are summarized in Table IV-6. Following Wilson's (1972) observations, bedform wavelength is assumed to be roughly 15 times dune height, which was calculated using the empirical relationship between bedform height and grainflow stratum thickness (after Kocurek and Nielson, 1986). Dune width and length were then estimated using the empirical relationships established by Breed and Grow (1979, p. 274) for crescentic bedforms. Reported values should be treated as first-order approximations; a $1-\mathrm{cm}$ difference in the reported grainflow stratum thickness translates into a difference in the other calculated dimensions of hundreds of meters. For C2 and B24-4a, estimates of original dune length and wavelength are based on direct measurements 


\begin{tabular}{|c|c|c|c|c|c|c|c|c|c|}
\hline UNIT & $A 8$ & $B 1$ & 816,818 & $\overline{B 24}-4 a$ & $B 13$ & $c 2(11)$ & C-COIPLEX & $\overline{02 / 08}$ & EI \\
\hline $\begin{array}{l}\text { WIDTH }(\mathrm{m} .,+10 \mathrm{~m} .) \\
\text { (Ditect measurement) } \\
\text { WIDTH }(\mathrm{m} .,+50 \mathrm{~m} .) \\
\text { (Breed and (irow, 1979) }\end{array}$ & & & & 150 & 1800 & 140 & $7-350$ & 100 & 40 \\
\hline $\begin{array}{l}\text { LFNGTH }(\mathrm{m} .,+50 \mathrm{~m}) \\
\text { (Breed and Grow, } 1979)\end{array}$ & & & & $100-200$ & 1100 & $50-200$ & $0-50$ & $<50$ & $?$ \\
\hline $\begin{array}{l}\text { WAVELENGTH }(\mathrm{m} .,+50 \mathrm{~m}) \\
\text { (Breed and Grow, 1979) } \\
\text { WAVELENGIH }(\mathrm{m} .+50 \mathrm{~m}) \\
\text { (WIIson, 1972) }\end{array}$ & & & & 500 & 1500 & 500 & $250-600$ & 450 & 300 \\
\hline $\begin{array}{l}\text { GRAINFLOW THICKNESS }(\mathrm{cm} .) \\
\text { DIrecl meas. }+0.5 \mathrm{~cm} . \\
\text { HE IGHI }(\mathrm{m} .,+5 \mathrm{~m} .) \\
\text { (kocuret and NIelson, in press) }\end{array}$ & $\begin{array}{l}3 \\
15\end{array}$ & $\begin{array}{l}3-4 \\
15-20\end{array}$ & $\begin{array}{l}3-4 \\
15-20\end{array}$ & $3-4$ & $11 \max$ & $20-30$ & $3-6$ & 30 & 20 \\
\hline HE IGHT:WAVELENGTH RAIIO & & & & $1: 25$ & $(1: 15)$ & $1: 17$ & $(1: 15)$ & $(1: 15)$ & $(1: 15)$ \\
\hline $\begin{array}{l}\text { SE ASONAL PACKAGE THICKNFSS } \\
\text { (birect measurement, }+5 \mathrm{~cm} \text { ) }\end{array}$ & $25-30$ & & 20 & & $20-40$ & $10-15$ & $10-20$ & $15-20$ & \\
\hline
\end{tabular}

Table IV-6. Dimensions of some of the bedforms represented by units of the study knob. 
of dune width (Table IV-6). The lens-shaped interdune deposit I1, measured perpendicular to the mean dip direction of overlying $\mathrm{C} 2$ strata, approximates the minimum possible width of the bedform represented by $\mathrm{C} 2$. Calculated ratios of $\mathrm{B} 24-4 \mathrm{a}$ and $\mathrm{C} 2$ dune height to wavelength compare favorably with Wilson's 1:15 ratio (Table IV-6).

Draa Geometry - The relative orientation of the components of the compound bedform represented by the B-complex were determined using Rubin and Hunter's (1983) suggested methods (Table IV-2, Fig. IV-25). Superimposed dunes migrated along as well as down the lee face of the draa; whether dunes actually migrated obliquely upwards, as is shown for $B 1$ and $B I 1$, is debatable. By not rotating their respective bounding surfaces 180 degrees to dip in the opposite direction, superimposed dunes of B14 and B15 (those marked with a double set of parentheses in Table IV-2; compare with Figure IV-18a) would have been calculated to have migrated almost directly upslope. Such a rotation is deemed justified by noting that bedforms migrating parallel to the local airflow direction should theoretically scour a bounding surface whose strike parallels the normal to the migration direction but may dip either way. That such assumptions need be made, emphasizes that interpretation of the deposits of the B-complex should be made conservatively. Moreover, most of the second-order bounding surfaces of the B-complex dip at 15 degrees or less (Table IV-2), rendering the assumption that bounding surface orientation approximates draa orientation less reliable (Rubin and Hunter, 1983).

A reconstruction of the deposits of the B-complex nonetheless 


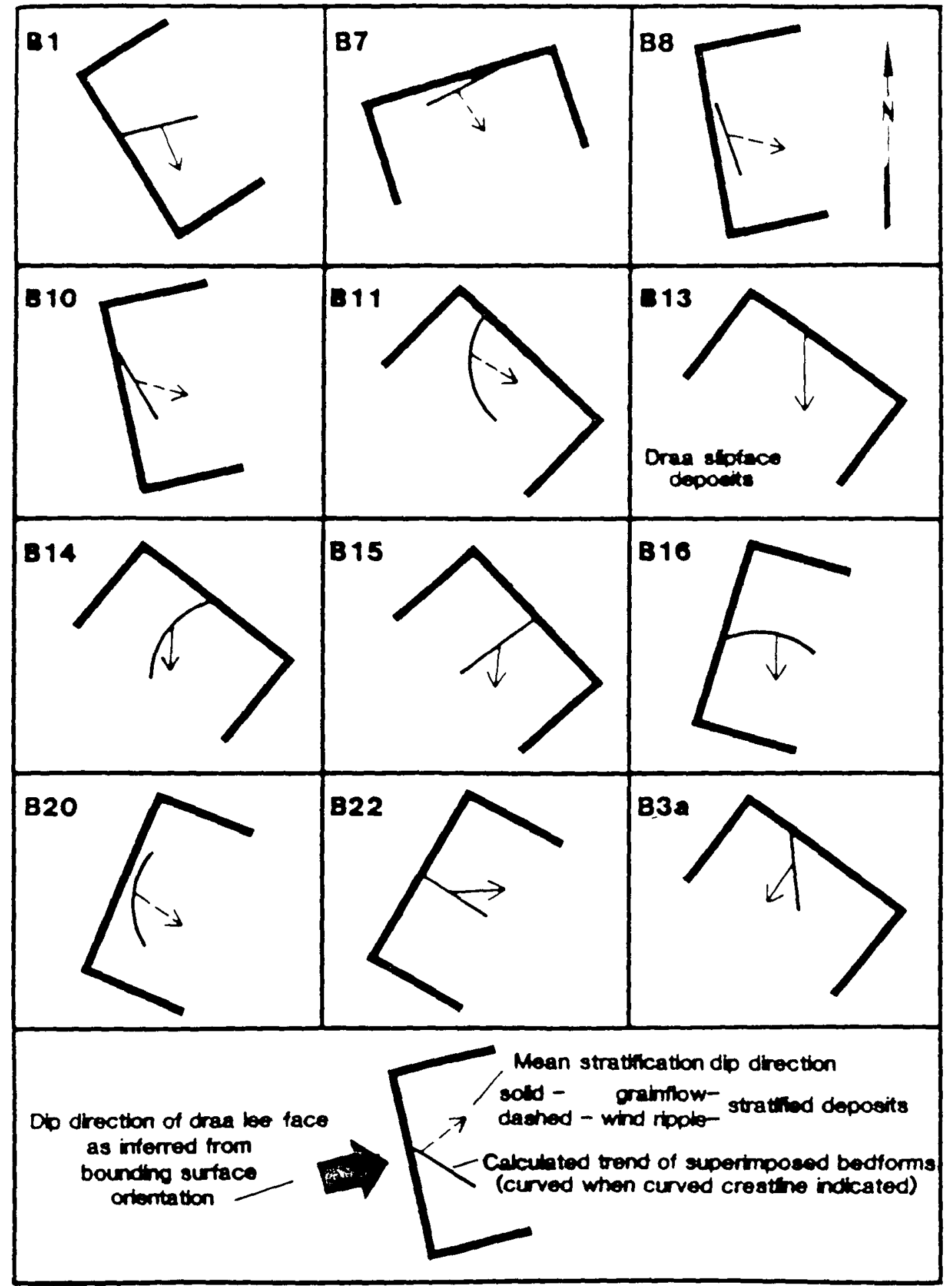

Figure IV-25. The relative orientation of the primary (draa) slipface to superimposed dune crests and stratification for units of the B-complex. Follows methods outlined by Rubin and Hunter (1983). 
reveals detail heretofore undocumented in ancient eolian sequences:

the importance of secondary airflow in depositing compound cross-bedding is documented by the oblique orientation of both superimposed dunes and stratification relative to the primary (main) slipface. Additionally, the primary slipface deposits of $\mathrm{B} 1 \mathrm{a}$ and $\mathrm{B} 13$ are distinguished from the other units of the complex by their relatively thick grainflow strata deposited almost directly down the draa slipface (Fig. IV-3, IV-25). While primary slipface deposits dip to the south, possibly identifying the dominant dip direction of the draa, a shifting draa slipface orientation is indicated by the variably directed second-order surfaces. The continuation of bounding surface trends through three or more successive units of the B-complex suggests that shifts in the draa slipface did in fact occur, probably the result of a curved draa slipface. The larger-scale cyclicity of reworked grainflow deposits observed in Bla supports this contention.

Bedform Morphodynamics - Most of the units that comprise the study area exhibit well-developed apron deposits and are therefore interpreted to represent dunes oriented oblique to the long-term sand-transport direction. Notable exceptions include the grainflow deposits of A8, B1a, C4, D2/D8, E1, and E14 (Fig. IV-14 through IV-17), which are inferred to have been deposited by at least locally transverse forms. The relationship between crestine curvature and morphodynamic dune type is not clearly expressed in the studied deposits; not all transverse bedform deposits are conclusively three-dimensional. For example, $\mathrm{C} 4$ shows the tightest distribution of stratification dip directions of all the units (Figure IV-18a), yet 
the associated bounding surface is the only nonplanar bounding surface of the C-complex. Many oblique dune deposits likewise show some evidence of a curved bedform shape. However, indications are commonly limited to the presence of the poorly defined, larger-scale cyclicity of reworked grainflow strata, whose origin remains undocumented.

Figure IV-26 attempts to establish a correlation between the presence/absence of basal apron deposits and the degree of angular divergence between stratification and bounding surface dip directions. Unfortunately, the relative lack of transverse dune deposits that comprise the local sequence limits interpretation by restricting the data base. What the histogram does show is that for units of the study area, the strata of oblique bedforms (those with wind-rippled apron deposits) may show any directional relationship with the corresponding bounding surface, while sand-sheet and transverse dune deposits tend to roughly parallel bounding surface orientation.

Migration Rates - Packages of grainflow strata bounded by thin but distinct wind-ripple laminae are the most common expression of small-scale cyclicity present within the sequence studied. This cyclicity is attributed to seasonal fluctuations in wind direction and/or strength, and can therefore be used to estimate the mean rate of dune advance. Rates based on measurements of package thickness made on a unit-by-unit basis vary from 10 to $40 \mathrm{~cm}$ per year (Table IV-6)

Summary and Conclusions - The tools for bedform reconstruction and interpretation draw mostly from the details of stratification noted on 
APRONLESS DUNE DEPOSITS

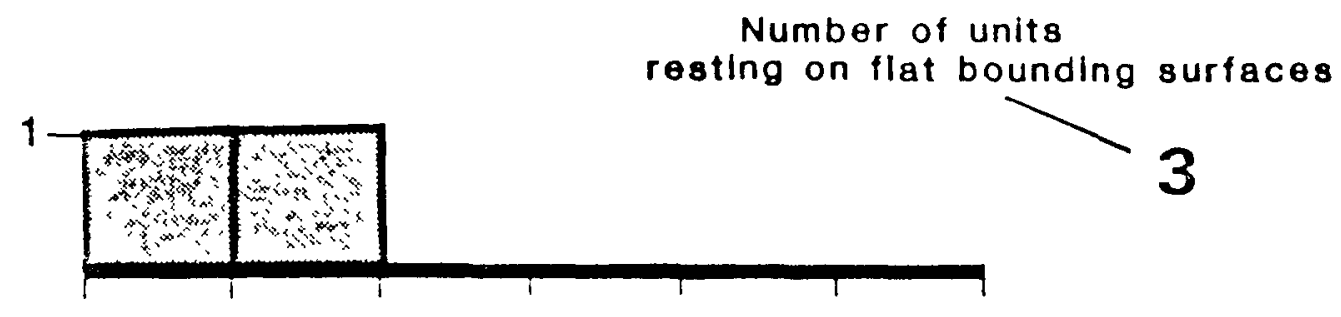

DUNE APRON DEPOSITS

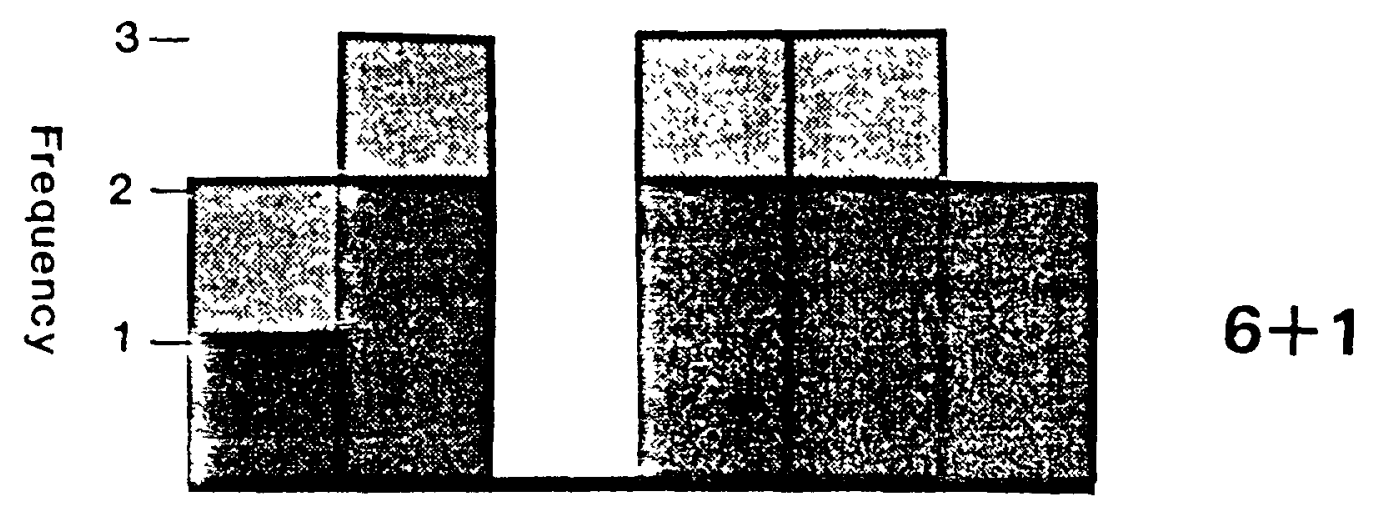

SAND SHEET DEPOSITS

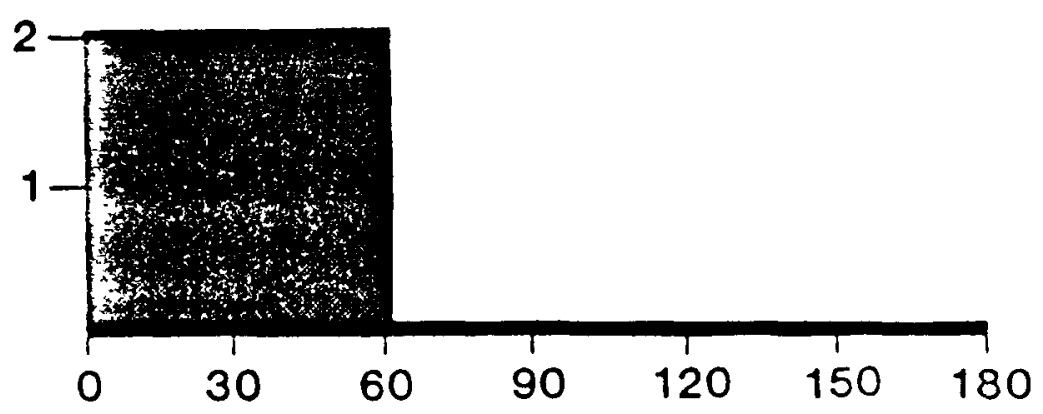

Angular difference between bounding surface orientation and stratification dip direction (degrees)

Figure IV-26. Histogram expressing the angular difference between bounding surface and stratification dip directions for units with and without apron deposits, and for sand sheets. Actual values for specific units are recorded in the 1ast column of Tables IV-1 through IV-5. 
a unit-by-unit basis. The bounding surfaces that delineate units within an ancient eolian sequence potentially reveal much, although their erosional character limits their usefulness. Indeed, their presence attests to the very small fraction of the original bedforms actually preserved. To this, add the complexity of eolian depositional environments as observed in modern sand seas, and it becomes clear that bedform reconstruction at present is limited to first-order generalizations. Nonetheless, an overall picture of the distribution of eolian subenvironments, of dune size and shape, and of the dynamics of eolian bedforms ean be at least theoretically derived. The degree to which present knowledge of eolian depositional systems can reconstruct and interpret ancient eolian sequences is revealed in the final interpretation of the sequence of the Page Sandstone described in detail above (Figure IV-24):

1. As deposition of the Page Sandstone commenced, coarse sands transported under relatively variable winds accumulated in local depressions in the $\mathrm{J} 2$ unconformable surface. These discontinuous sand-sheets are represented locally by the A-complex. Isolated barchanoid dunes 10 to 15 meters high (exemplified by the deposits of A8) migrated southwards across the Page sand-sheet. Improved directionality in the surface wind pattern and possibly an increased sand supply, accompanied a gradual infilling of J2 topography. Variably directed wind-ripple strata are succeeded in the A-complex by deposits of moderately dipping, slipfaceless zibars migrating eastward.

2. The compound dune or draa represented by the B-complex subsequently truncated the basal sand-sheet deposits and locally 
marked the end of the hiatus in sand-sea development represented by J2. The draa was a slightly curved form standing rough1y $100 \mathrm{~m}$ high, whose main slipface dipped to the south, parallel to the primary wind direction. Barchanoid-shaped superimposed bedforms, 15 to $20 \mathrm{~m} \mathrm{high}$, migrated alongslope and obliquely downslope, possibly controlled by secondary airflow blowing consistently eastward along the lee face of the draa.

3. The bedforms that deposited the succeeding C-complex were comparatively linear, simple (?) dunes roughly $40 \mathrm{~m}$ high, commonly exhibiting we11-developed wind-rippled aprons. Dunes of the C-complex trended obliquely to the long-term resultant sand-transport direction, although stratification dipping consistently to the south and southeast indicate a unimodal pattern of sand-transporting winds. Interdune areas were either deflationary or the site of wind-ripple deposition under dry condtions.

Transgression of the Carmel seaway from the northwest interrupted local eolian conditions represented by the C-complex, and deposited the clayey sediments of the Judd Hollow tongue. C1l, which overlies the tongue of the Carmel and is identified by its slightly coarser texture, can be traced miles beyond the study area, suggesting that the receding tongue of the Carmel seaway exposed large amounts of sand that was subsequently reworked into dunes of the Page erg.

Polygonally fractured regional bounding surfaces at the top of the C-complex 1ikewise mark periods when marginal marine conditions encroached upon the area, briefly interrupting sand sea deposition. Associated with these regional bounding surfaces, abundant reactivation surfaces and an isolated trough-shaped unit indicatate 
variable, occasionally strong winds; marginal marine conditions during Page deposition remained close enough to the study site to overprint the prevailing wind regime with a complex coastal wind pattern.

4. The poorly exposed units of the D-complex were probably deposited by a compound bedform that stood at least $30 \mathrm{~m}$ high. The main slipface of the draa, like the draa of the B-complex, dipped to the south and was roughly transverse to sand-transport direction. Variable winds and the continued influence of nearby marginal marine conditions are indicated by the relative abundance of reactivation surfaces in D2/D8. Polygonally fractured horizons in the E-complex represent truncation of the Page erg by evaporite-encrusted plains, and confirm that marginal marine conditions remained close enough to the erg at the study site to influence deposition. The dunes represented by the units of the uppermost E-complex were three-dimensional bedforms at least $20 \mathrm{~m} \mathrm{tall,} \mathrm{oriented} \mathrm{obliquely} \mathrm{to}$ the south/southeast sand transport direction. The crescentic bedforms of the E-complex were generally smaller than those of the C-complex, supporting the contention that at the study site, the Page erg shifted to a more marginal position, relatively close to coastal conditions. 


\begin{tabular}{|c|c|c|c|c|c|c|c|c|c|c|}
\hline UNIT/SURFACE & $\begin{array}{l}\text { Sample } \\
\text { Sizo }\end{array}$ & Order & $\begin{array}{c}X(N-S) \\
E-02\end{array}$ & $\begin{array}{c}Y(E-W) \\
E-02\end{array}$ & $\begin{array}{c}X Y \\
E-03\end{array}$ & $\begin{array}{c}X X \\
E-03\end{array}$ & $\begin{array}{c}Y Y \\
E-03\end{array}$ & Constant & $\begin{array}{l}\text { Coeffletent of } \\
\text { Determination }\end{array}$ & $\begin{array}{l}\text { Standard } \\
\text { Doviation }\end{array}$ \\
\hline \multirow{2}{*}{$\begin{array}{l}\mathrm{J} 2 \text { unconform. } \\
\text { (Polygons) }\end{array}$} & 34 & 1 & 1.500 & 2.128 & & & & 1.271 & 0.956 & 0.17 \\
\hline & & 2 & 2.799 & 2.340 & -0.320 & -0.063 & -0.195 & 1.933 & 0.991 & 0.11 \\
\hline \multirow[t]{2}{*}{ A4 } & 11 & 1 & 0.278 & 0.020 & & & & 1.211 & 0.113 & 0.06 \\
\hline & & 2 & -27.310 & -32.10 & -8.872 & -3.706 & -5.568 & -3.923 & 0.679 & 0.04 \\
\hline \multirow[t]{2}{*}{ AS } & 7 & 1 & 3.350 & 0.185 & & & & 3.730 & 0.849 & 0.11 \\
\hline & & 2 & 16.964 & -22.176 & -3.231 & & & 13.263 & 0.991 & 0.03 \\
\hline \multirow[t]{2}{*}{ A6 } & 21 & 1 & 2.054 & 3.484 & & & & 1.723 & 0.615 & 0.19 \\
\hline & & 2 & -19.88 & -18.93 & -6.344 & -2.724 & -4.363 & -2.842 & 0.963 & 0.06 \\
\hline \multirow[t]{2}{*}{ A? } & 15 & 1 & 0.476 & 3.892 & & & & 1.075 & 0.989 & 0.04 \\
\hline & & 2 & -11.342 & -3.668 & -1.987 & -1.393 & -0.938 & -1.426 & 0.996 & 0.02 \\
\hline \multirow[t]{2}{*}{ AO } & 6 & 1 & 5.308 & 5.063 & & & & 3.966 & 0.677 & 0.12 \\
\hline & & 2 & 23.472 & 154.88 & & & -15.240 & -16.012 & 1.000 & 0.00 \\
\hline \multirow[t]{2}{*}{ Ag } & 17 & 1 & -0.803 & 0.822 & & & & 0.970 & 0.622 & 0.21 \\
\hline & & 2 & 0.510 & 6.560 & 0.274 & 0.22 & -1.095 & 0.845 & 0.955 & 0.07 \\
\hline \multirow[t]{2}{*}{ A 10} & 14 & 1 & 0.121 & -4.031 & & & & 3.626 & 0.970 & 0.07 \\
\hline & & 2 & -8.355 & -0.870 & 0.775 & -0.908 & 1.301 & 1.667 & 0.993 & 0.03 \\
\hline \multirow[t]{2}{*}{ All } & 15 & 1 & 0.113 & -4.606 & & & & 4.106 & 0.966 & 0.11 \\
\hline & & 2 & -13.270 & -29.310 & -6.482 & -2.224 & -3.686 & 2.681 & 0.988 & 0.06 \\
\hline \multirow[t]{2}{*}{ B1 } & 8 & 1 & 4.840 & -7.681 & & & & 9.765 & 0.994 & 0.05 \\
\hline & & 2 & 6.782 & -47.04 & $-2.97 \overline{0}$ & & & 17.237 & 0.999 & 0.00 \\
\hline B7 & 5 & 1 & 1.276 & 0.421 & & & & 2.740 & 0.970 & 0.01 \\
\hline$B 8$ & 5 & 1 & 18.85 & 49.91 & & & & 50.050 & 0.682 & 0.40 \\
\hline 810 & 4 & 1 & 14.98 & -69.19 & & & & 64.350 & 0.945 & 0.07 \\
\hline BII & 4 & 1 & -26.187 & 24.872 & & & & -32.658 & 0.999 & 0.01 \\
\hline \multirow[t]{2}{*}{813} & 7 & 1 & -41.325 & 30.688 & & & & -46.330 & 0.956 & 0.09 \\
\hline & & 2 & -162.39 & 120.23 & 20.810 & & & -91.194 & 0.999 & 0.01 \\
\hline 814 & 4 & 1 & 10.031 & -7.757 & & & & 14.762 & 0.824 & 0.01 \\
\hline
\end{tabular}

Appendix. The results of trend surface analysis. The bounding surface described lies below the unit by which it is identified. The origin is in the northwest corner. Data entered were in feet from the origin, divided by ten. Polygonally fractured surfaces are identified as such. 


\begin{tabular}{|c|c|c|c|c|c|c|c|c|c|c|}
\hline UNIT /SURFACE & $\begin{array}{l}\text { Samplo } \\
\text { SIze }\end{array}$ & Order & $\begin{array}{c}X(N-S) \\
E-02\end{array}$ & $\begin{array}{c}Y(E-W) \\
E-02\end{array}$ & $\begin{array}{c}X Y \\
E-03\end{array}$ & $\begin{array}{c}x X \\
E-03\end{array}$ & $\begin{array}{c}Y Y \\
E-03\end{array}$ & Constant & $\begin{array}{l}\text { Coofficiont of } \\
\text { Determination }\end{array}$ & $\begin{array}{l}\text { Standard } \\
\text { Doviation }\end{array}$ \\
\hline 815 & 6 & 1 & 23.110 & -25.519 & & & & 36.889 & 0.971 & 0.05 \\
\hline B 16 & 6 & 1 & -2.632 & -10.072 & & & & 9.747 & 0.996 & 0.02 \\
\hline 820 & 4 & 1 & -11.010 & -26.860 & & & & 21.49 & 0.691 & 0.12 \\
\hline 822 & 1 & 1 & -10.786 & -19.237 & & & & 15.147 & 0.999 & 0.00 \\
\hline \multirow[t]{2}{*}{ B3o } & 10 & 1 & -22.221 & 16.209 & & & & & 0.944 & 0.10 \\
\hline & & 2 & 11.810 & 27.594 & 12.256 & 5.125 & & 3.220 & 0.993 & 0.04 \\
\hline \multirow[t]{2}{*}{11} & 13 & 1 & -0.058 & -2.181 & & & & 4.836 & 0.989 & 0.03 \\
\hline & & 2 & 0.997 & -2.466 & -1.294 & -6.61 & & 5.228 & 0.997 & 0.02 \\
\hline \multirow[t]{2}{*}{$\mathrm{C2}$} & 44 & 1 & 0.315 & -1.412 & & & & 4.570 & 0.703 & 0.22 \\
\hline & & 2 & & & & & & & 0.849 & 0.16 \\
\hline \multirow[t]{2}{*}{$\mathrm{C3}$} & 10 & 1 & 4.216 & -1.039 & & & & 8.234 & 0.964 & 0.10 \\
\hline & & 2 & 1.700 & 25.190 & 0.021 & -0.308 & -2.411 & 1.524 & 0992 & 0.05 \\
\hline 12 & 9 & 1 & 1.790 & 2.728 & & & & 5.827 & 1.000 & 0.00 \\
\hline \multirow[t]{2}{*}{$C_{4}$} & 12 & 1 & 0.530 & & & & & 5.550 & 0.258 & 0.09 \\
\hline & & 2 & 0.948 & 2.073 & 0.762 & 0.896 & 0021 & 5.069 & 0.949 & 0.03 \\
\hline \multirow[t]{2}{*}{$C 7-20$} & 14 & 1 & 1.657 & 0.534 & & & & 6.548 & 0.953 & 007 \\
\hline & & 2 & 2.842 & -1.243 & -0.488 & -0.07 & 0.002 & 7.231 & 0.995 & 0.04 \\
\hline \multirow[t]{2}{*}{ C3b } & 6 & 1 & 1.975 & 5.731 & & & & 6.210 & 0944 & $\begin{array}{ll}0 & 01\end{array}$ \\
\hline & & 2 & 1.442 & 10.586 & 0.878 & & & 5.901 & 1.000 & 0.00 \\
\hline \multirow[t]{2}{*}{$\mathrm{CB}$} & 14 & 1 & 1.699 & 0.847 & & & & 6.458 & 0.775 & 0.08 \\
\hline & & 2 & -7.544 & 3.269 & -0.687 & -2.546 & -0.406 & 4.821 & 0997 & 0.01 \\
\hline \multirow[t]{2}{*}{$C 9$} & 9 & 1 & 2.421 & 0.010 & & & & 7.688 & 0.787 & 005 \\
\hline & & 2 & 9.363 & -8.987 & -1.847 & -0.211 & 0.557 & 10.338 & 0.992 & 002 \\
\hline \multirow[t]{2}{*}{$C 10$} & 6 & 1 & -4.493 & 2.240 & & & & 5.429 & 0.991 & 003 \\
\hline & & 2 & -0.280 & -0.608 & -1.115 & & & 6.516 & 0.993 & 004 \\
\hline \multirow[t]{2}{*}{$\mathrm{Cll}$} & 31 & 1 & 3.789 & -3.404 & & & & 10.189 & 0.975 & 0.16 \\
\hline & & 2 & 6.910 & -5.129 & -0.461 & -0.059 & -0.002 & 11.263 & 0.984 & 0.13 \\
\hline
\end{tabular}

Appendix (continued). 


\begin{tabular}{|c|c|c|c|c|c|c|c|c|c|c|}
\hline UNIT/SURF ACE & $\begin{array}{l}\text { Samplo } \\
\text { Size }\end{array}$ & Order & $\begin{array}{c}x(N-S) \\
E-02\end{array}$ & $\begin{array}{c}Y(E-W) \\
E-02\end{array}$ & $\begin{array}{c}X Y \\
E-03\end{array}$ & $\begin{array}{c}x x \\
E-03\end{array}$ & $\begin{array}{c}Y Y \\
E-03\end{array}$ & Constant & $\begin{array}{l}\text { Coefficiont of } \\
\text { Dotormination }\end{array}$ & $\begin{array}{l}\text { Standard } \\
\text { Deviation }\end{array}$ \\
\hline \multirow[t]{2}{*}{$\mathrm{C} 12$} & 7 & $\mathbf{I}$ & 3.496 & 5.200 & & & & 7.370 & 0.748 & 0.32 \\
\hline & & 2 & -6.130 & 6.533 & 5.816 & 0.316 & 6.976 & 4.282 & 0.994 & 0.05 \\
\hline $\mathrm{Cl3}$ & 31 & 1 & 2.448 & 0.221 & & & & 8.733 & 0.984 & 0.06 \\
\hline (Polygons) & & 2 & 3.859 & 0.336 & -0.003 & 0.135 & -0.017 & 9.034 & 0.990 & 0.05 \\
\hline Polygonal surf. & 30 & 1 & 2.094 & 0.307 & & & & 9.149 & 0.981 & 0.05 \\
\hline abovo C13 & & 2 & 2.548 & 1.367 & 0.009 & 0.042 & -0.119 & 9.082 & 0.988 & 0.04 \\
\hline D6 & 4 & 1 & 10.480 & -15.80 & & & & 19.47 & 0.932 & 0.07 \\
\hline D10 & 5 & 1 & 6.334 & 38.704 & & & & 1.726 & 0.998 & 0.03 \\
\hline DII & 4 & 1 & 6.128 & -12.879 & & & & 15.777 & 0.996 & 0.01 \\
\hline \multirow[t]{2}{*}{ D12 } & 8 & 1 & 4.089 & 14.027 & 、 & & & 7.658 & 0.894 & 0.24 \\
\hline & & 2 & -30.177 & -25.884 & -3.213 & -5.412 & -6.791 & 5.293 & 0.997 & 0.08 \\
\hline \multirow[t]{2}{*}{ E4 } & 7 & 1 & 6.159 & 0.301 & & & & 13.130 & 0.561 & 0.13 \\
\hline & & 2 & -38.350 & 53.840 & 7.904 & & & -16.930 & 0.874 & 0.10 \\
\hline \multirow[t]{2}{*}{ E5 } & 10 & $i$ & -1.571 & -6.106 & & & & 11.714 & 0.506 & 0.37 \\
\hline & & 2 & 13.306 & -91.44 & -1.212 & 0.811 & 8.005 & 36.111 & 0.970 & 0.09 \\
\hline \multirow[t]{2}{*}{ E6 } & 11 & 1 & 6.702 & 6.863 & & & & 10.665 & 0.876 & 0.16 \\
\hline & & 2 & 113.36 & -103.48 & -11.370 & 5.962 & 3.882 & 63.100 & 0.982 & 0.06 \\
\hline Polygonal surf. & 9 & 1 & 2.050 & 0.632 & & & & 10.889 & 0.891 & 0.06 \\
\hline bolow E7 & & 2 & 7.117 & 1.839 & 0.182 & 0.535 & & 11.915 & 0.966 & 003 \\
\hline Polygonal surf. & 6 & 1 & 1.915 & & & & & 11.166 & 0.909 & 0.02 \\
\hline Dolow E9 & & 2 & 2.159 & 0.197 & & & & 11.184 & 1.000 & 0.00 \\
\hline Theso two po & 1 & racos & - angrar & oarallel & & & & o the the & ie tIme ll & \\
\hline
\end{tabular}

Appendix (continued). 


\begin{tabular}{|c|c|c|c|c|c|c|c|c|c|c|}
\hline UNIT/SURFACE & $\begin{array}{c}\text { Samplo } \\
\text { Sizo }\end{array}$ & Order & $\begin{array}{c}X(N-S) \\
E-02\end{array}$ & $\begin{array}{c}Y(E-W) \\
E-02\end{array}$ & $\begin{array}{c}X Y \\
E-03\end{array}$ & $\begin{array}{c}x x \\
E-03\end{array}$ & $\begin{array}{c}Y Y \\
E-03\end{array}$ & Constant & $\begin{array}{l}\text { Coefficlent of } \\
\text { Determination }\end{array}$ & $\begin{array}{l}\text { Standard } \\
\text { Deviation }\end{array}$ \\
\hline Polygonal surt. & 11 & $!$ & 0.598 & -0.165 & & & & 10.886 & 0.124 & 0.18 \\
\hline above E7 & & $\dot{2}$ & -34.90 & -21.04 & 4.424 & -1.111 & 4.377 & 6.474 & 0.875 & 0.06 \\
\hline \multirow[t]{2}{*}{ E10 } & 6 & 1 & 0.343 & -0.787 & & & & 11.450 & 0.432 & 0.05 \\
\hline & & 2 & -28.02 & 24.60 & 5.992 & -0.526 & & 0.405 & 1.000 & 0.00 \\
\hline \multirow[t]{2}{*}{ E 12} & 18 & $i$ & 2.480 & 2.148 & & & & 11.219 & 0.959 & 0.03 \\
\hline & & 2 & 3.984 & 0.092 & -0.258 & 0.019 & 0.094 & 12.061 & 0.971 & 0.03 \\
\hline \multirow[t]{2}{*}{ E 13} & 9 & 1 & -0.193 & -0.764 & & & & 11.516 & 0.102 & 0.11 \\
\hline & & 2 & 29.51 & 23.19 & 6.810 & 5.756 & 1.359 & 13.697 & 0.504 & 0.08 \\
\hline \multirow[t]{2}{*}{ E14 } & 16 & 1 & -1.229 & -2.528 & & & & 11.930 & 0.296 & 0.13 \\
\hline & & 2 & 4.991 & 22.544 & 5.145 & 3.104 & & 7.613 & 0.563 & 010 \\
\hline
\end{tabular}

Appendix (continued). 
PART IV. REFERENCES

Ahlbrandt, T.S., "Textural Parameters of Eolian Deposits," A Study of Global Sand Seas, (Ed. by E.D. Mckee), Prof. Pap. U.S. Geol. Surv., (1979) 1052, 21-51.

AhIbrandt, T.S. and Fryberger, S.G. "Introduction to Folian Sediments," Sandstone Depositional Environments, (Ed. by P.A. Scholle and D. Spearing), Am. Asso.Petrol. Geol. Memoir 31, (1982) 11-48.

Bagnold, R.A., The Phrsics of Blown Sand and Desert Dunes, Methuen, London, (1941) $265 \mathrm{pp}$.

Blakey, R.C. and Middleton, L.T. "Permian Shoreline E lian Complex in Central Arizona: Dune Changes in Response to Cyclic Sea-level Changes, Eolian Sediments and Processes, (Ed. by M.E. Brookfield and T.S. AhIbrandt) (1983) 551-581.

Blakey, R.C., Peterson, F., Caputo, M.V., Geesaman, R.C., and Voorhees, B.J., "Paleogeography of Middle Jurassic Continental, Shoreline, and Shallow Marine Sedimentation, Southern Utah," Mesozoic Paleogeography of West-Central United States, (Ed. by M.W. Reynolds and E.D. Dolly), Soc. Econ. Paleont. Miner. Rocky Mtn. Section, (1983) 77-100.

Breed, C.S. and Grow, T., "Morphology and Distribution of Dunes in Sand Seas Observed by Remote Sensing," A Study of Global Sand Seas, (Fd. by E.D. McKee), Prof. Pap. U.S. geol. Surv. 1052, 253-302.

Brookfield, M.E., "The Origin of Bounding Surfaces in Ancient Aeolian Sandstones," Sedimentology 24, (1977) 303-332.

Brookfield, M.E., "Eolian Sands," Facies Models (Ed. by R.G. Walker), Geoscience Canada Reprint Series 1, (1984) 91-103.

Caputo, M.V., "Depositional History of Middle Jurassic Clastic Shoreline Sequences in Southeastern Utah and Southwestern Colorado. M.S. thesis, Department of Geology, Northern Arizona University, Flagstaff (1980).

Chandler, M.A. and Kocurek, G., "Depositional Controls on Permeability in an Eolian Sandstone Sequence, Page Sandstone, Northern Arizona," (in preparation) Bu11. Am. Ass. Petrol. Geol., 1986.

Chorley, R.J. and Haggett, P., "Trend Surface Mapping in Geographical Research," Transactions and Papers of the Institute of British Geographers 37, (1965) 47-67.

Davis, J.C., Statistics and Data Analysis in Geology, John Wiley \& Sons, New York, (1973) 550 pp. 
Driese, S.G. and Dott, R.H. Jr., "Model for Sandstone-Carbonate Cvclothems Based on Upper Member of Morgan Formation (Middle Pennsylvanian) of Northern Utah and Colorado," Bull. Am. Ass. Petro1. Geol., 68, (1984) 574-597.

Folk, R.L., "Bimodal Supermature Sandstones: Product of the Desert Floor," Genesis and Classification of Sedimentary Rocks: Report of the Twenty-third Session of the International Geological Congress (Prague), (1968) 9-32.

Folk, R.L. Longitudinal Dunes of the Northwestern Edge of the Simpson Desert, Northern Territory, Australia, 1. Geomorphology and Grain Size Relationships. Sedimentology 16, (1971) 5-54.

Fryberger, S.G. "Dune Forms and Wind Regime," A Study of Global Sand Seas (Ed. by E.D. McKee), Prof. Pap. U.S. Geol. Surv. 1052, (1979) 137-169.

Goggin, D.J., Chandler, M.A., Lake, L.W., and Kocurek, G. "Patterns of Permeability in Eolian Deposits," (abs). Soc. Pet. Eng./Dept. of Energy Fifth Symposium on Enhanced Oil Recovery, Tulsa, (1986).

Havholm, K., "Dynamics of a Modern Draa, Algodones Dune Field, California," M.A. thesis, Department of Geological Sciences, University of Texas, Austin (1986).

Holm, D.A., "Desert Geomorphology in the Arabian Peninsula," Science 132, (1960) 1369-1379.

Hunter, R.E. "Basic Types of Stratification in Small Eolian Dunes," Sedimentology 24, (1977) 361-387.

Hunter, R.E., "Stratification Styles in Eolian Sandstones: Some Pennsylvanian to Permian Examples from the Western Interior U.S.A.," Recent and Ancient Nonmarine Depositional Systems: Models for Exploration (Ed. by F.G. Ethridge and R.M. Flores), Soc. Eecon. Paleont. Miner. Spec. Pub. 31, (1981) 315-329.

Hunter, R.E., Richmond, B.M. and Alpha, T.R., "Storm-controlled Oblique Dunes of the Oregon coast," Bull. Geol. Soc. Am.. 94, (1983) 1450-1465.

Hunter, R.E. and Rubin, D.M., "Interpreting Cyclic Cross-bedding, with an Example from the Navajo Sandstone," Eolian Sediments and Processes (Ed. by M.E. Brookfield and T.S. Ahlbrandt), (1983) $429-454$.

Hunter, R.E. and Rubin, D.M., "Interpreting Eolian Cross-strata: the Limits of the Possible," (abs). Soc. Econ. Paleont. Miner. Ann. Midyear Meeting, Golden, (1985) p. 44.

Kocurek, G., "Significance of Interdune Deposits and Bounding Surfaces in Aeolian Dune Sands," Sedimentology 28, (1981a) 753-780. 
Kocurek, G., "Erg Reconstruction: The Entrada Sandstone (Jurassic) of Northern Utah and Colorado. Palaeogeog. Paleaeoclim. Palaeoecol. 36 , (1981b) 125-153.

Kocurek, G., "Origin of First-order Bounding Surfaces in Aeolian Sandstones: Reply," Sedimentology 31, (1984) 125-127.

Kocurek, G., "Orfgins of Low-angle Stratification in Aeolian Deposits," Folian Geomorphology (Ed. by W. Nickling), Proceedings of the 17th Geomorphology Conference, Guelph, Canada (in press) (1986).

Kocurek, G. and Dott, R.H. Jr., "Distinctions and Uses of Stratification Types in the Interpretation of Eolian Sand," $\mathrm{J}$. Sedim. Petrol. 51, (1981) 579-595.

Kocurek, G. and Dott, R.H. Jr., "Jurassic Paleogeography and Paleoclimate of the Central and Southern Rocky Mountains Region," Mesozoic Paleogeography of liest-Central United States (Ed. by M.W. Reynolds and E.D. Dolly), Soc. Econ. Paleont. Miner. Rocky Mtn. Section, (1983) 101-116.

Kocurek, G. and Hunter, R.E., "Origin of Polygonal Fractures in Sand, Uppermost Navajo and Page Sandstones," J. Sedim. Petrol., (in press) 1986.

Kocurek, G. and Nielson, J., "Conditions Favorable for the Formation of Warm-climate Eolian Sand Sheets," Sedimentology, (1986a) in press.

Kocurek, G. and Nielson, I., "Estimation of Ancient Eolian Dune Height from Grainflow Cross-strata. J. Sedim. Petrol., (1986b) in press.

Kocurek, G. and Oakes, C., "Migration of Dunes and Ergs--Bounding Surfaces Revisited," (abs)., Soc. Econ. Paleont. Miner. Ann. Midyear Meeting, Golden, (1985), p. 50.

Lake, L. W., Miller, M. A. and Kocurek, G. A., Department of Energy Proposal, 1985.

Loope, D.B., "Deposition, Deflation and Diagenesis of Upper Paleozoic Eolian Sediments, Canyonlands National Park, Utah," Ph.D. dissertation, Department of Geology, University of Wyoming, Laramie, (1981).

Loope, D.B., "Origin of Extensive Bedding Planes in Aeolian Sandstones: A Defence of Stokes' Hypothesis," Sedimentology 31, (1984) 123-125.

McKee, E.D., "Introduction to a Study of Global Sand Seas," A Study of Global Sand Seas (Ed. by E.D. McKee), Prof. Pap. U.S. geol. Surv. 1052, (1979a) 1-19. 
McKee, E.D., "Sedimentary Structures in Dunes," A Study of Global of Sand Seas (Ed. by E.D. McKee), Prof. Pap. U.S. Geol. Surv. 1052, (1979b) 87-113.

McKee, E.D., "Ancient Sandstones Considered to be Eolian," A Study of Global Sand Seas (Ed. by E.D. McKee), Prof. Pap. U.S. Geol. Surv. $1052,(1979 \mathrm{c}) 187-238$.

McKee, E.D. and Tibbitts, G.C., Primary Sructures of a Seif Dune and Associated Deposits in Libya. J. Sedim. Petrol. 34, (1964) 5-17.

Nielson, J., "Linear Dunes of the Algodones Dune Field, Southeastern California, (in prep.), 1986.

Nielson, J. and Kocurek, G., "Climbing Zibars of the Algodones," Sedim. Geol. 48, $( \pm 986) 1-15$.

Peterson, F. and Pipiringos, G.N., "Stratigraphic Relations of the Navajo Sandstone to Middle Jurassic Formations, Southern Utah and Northern Arizona," Prof. Pap. U.S. Geol. Surv. 1035-B, (1979) pp. B1-B43.

Pipiringos, G.N. and O'Sullivan, R., "Principal Unconformities in Triassic and Jurassic Rocks, Western Interior United States- $A$ preliminary survey," Prof. Pap. U.S. Geol. Surv. 1035-A, (1978) Pp. A1-A29.

Poole, F.G., "Wind Directions in Late Paleozoic to Middle Mesozoic Time on the Colorado Plateau," Prof. Pap. U.S. Geol. Surv. 450-D, (1962) 147-151.

Porter, M.L., "Sedimentary Record of Erg Migration," Geology, 14, (1986) 497-500.

Reiche, P., "An Analysis of Cross-lamination--the Coconino Sandstone," J. Geo1., 46, (1938) 905-932.

Ross, G.M., "Bigbear Erg: Intermontaine Eolian Sand Sea in the Hornby Bay Group, Northwest Territories, Canada," Eolian Sediments and Processes (Ed. by M.E. Brookfield and T.S. Ahlbrandt), (1983) 483-519.

Rubin, D.M. and Hunter, R.E., "Bedform Climbing in Theory and Nature," Sedimentology 29, (1982) 121-138.

Rubin D.M. and Hunter, R.E., "Reconstructing Bedform Assemblages from Compound Cross-bedding," Eolian Sediments and Processes (Ed. by M.E. Brookfield and T.S. Ahlbrandt), (1983) 407-427.

Rubin, D.M. and Hunter, R.E., "Bedding Planes in Aeolian Sandstones: Another Reply," Sedimentology 28, (1984) 128-131. 
Rubin, D.M. and Hunter, R.E., "Why Deposits of Longitudinal Dunes are Rarely Recognized in the Geologic Record," Sedimentology 32, (1985) 147-157.

Simpson, E.L. and Loope, D.B., "Amalgamated Interdune Deposits, White Sands, New Mexico," J. Sedim. Petrol. 55, (1985) 361-365.

Stokes, S.L., "Multiple Paralle1-truncation Bedding Planes--feature of Wind Deposited Sandstone Formations," J. Sedim. Petrol. 38, (1968) 510-515.

Talbot, M.R., "Major Bounding Surfaces in Aeolian Sandstones--A Climatic Mode1," Sedimentology 32, (1985) 257-265.

Tsoar, H., "Dynamic Processes Acting on a Longitudinal (Seif) Sand Dune," Sedimentology 30, (1983) 567-578.

Wasson, R.J. and Hyde, R., "Factors Determining Desert Dune Type," Nature 304, (1983) 337-339.

Wilson, I.G., "Aeolian Bedforms- Their Development and Origins," Sedimentology 19, (1972) 173-210.

Wilson, I.G., "Ergs," Sedim. Geol. 10, (1973) 77-106. 


\section{PART V. PROCEDURE VALIDATION}

Easily the most nebulous step of reservoir characterization is a method for validating the procedure. The approach to validation adopted here is to model fluid flow in a specific heterogeneous medium both stochastically and deterministically and make technical comparisons of the results. Before doing this, we must procure a good deterministic description of the medium to be simulated. Such a description was the subject of Part IV of this report.

The stochastic simulation will require a statistical description of the subject reservoir and an efficient procedure for generating partially correlated random fields. The statistical description was reported previously (Goggin et a1., 1986; Chandler, 1986). In this part we report on efforts to generate stochastic permeability fields based on one-dimensional line processes and give results on pore-level modeling. The actual results of the simulation will be reported on in the next annual report. 
INCLUDING A STOCHASTIC OVERPRINT

Imagine that the statistical character of a reservoir is available through its distribution type, variance, and correlation function. Such information can be used to generate a stochastic line process. As discussed in Part III, the methods to generate these processes have inherent limitations for large correlation lengths and for non-stationary processes. Nevertheless, several line process can be merged through the turning bands method (TBM) to generate a two-dimensional stochastic field. The purpose of this section is to present some preliminary results on such fields. See Montoglou and Wilson (1976) for details on TBM.

For illustration we generate a two-dimensional field with mean of 100, coefficient of variation of 1 , and a sample span/correlation length of 20. The correlation model is exponential and the data are distributed according to the non-normal distribution of Part III with $\mathrm{p}=0.5$. We generate the field on a $50 \times 50$ grid each with length 2; thus, the actual correlation length must be 5 .

For a specific set of random numbers, the standard deviation Input to the line process generator must be 62 for the output standard deviation to be 100. This difference is because of the p-normal transformation of the data: had $p$ been unity the input standard deviation would have to be about 96. Similarly, the input correlation length must be 4.5 to obtain the output value of 5 . See Part III.

Figure $V-1$ shows the variograms in the $x$ and $y$ directions, respectively, calculated from the generated field. Both vartograms 


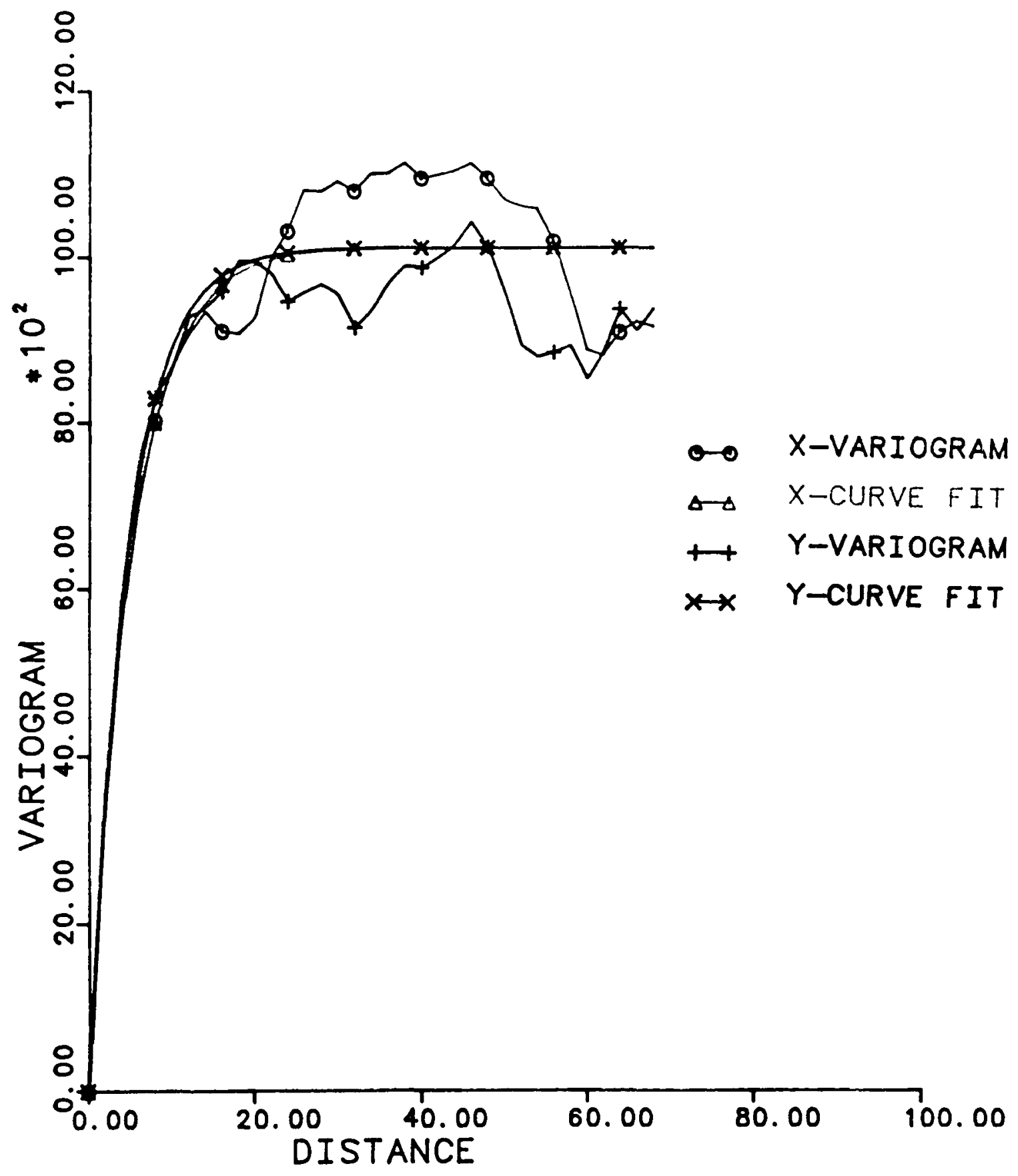

Figure V-1. Comparison of Input and Generated Variograms. 50X50, $\quad D X=D Y=2, \quad P=.5, \quad C O V=1$ 
are nearly the same (the field is isotropic in correlation) and both agree quite well with the input variogram (the smooth curve in Fig. $\mathrm{V}-1)$. The agreement is particularly good in the correlated portion of the variogram. Figure $\mathrm{V}-2$ shows the agreement between the input and calculated spectral density functions.

Figure V-3 shows the cumulative probability density plot expressed on a probabilistic x-axis. The y-axis plots the permeability values raised to the 0.5 power and then transformed to have unit variance and zero mean. The linearity of the plot and the agreement with the theoretical input (smooth curve) shows that the two-dimensional field generator is working properly. The sma11 deviation on the left of the plot is caused by omitting negative permeability values after they are generated. For the case considered these do not affect the function very much; however, they would be a cause for concern if the desired mean value were lower.

Figure V-4 shows a contour plot of the generated field. 


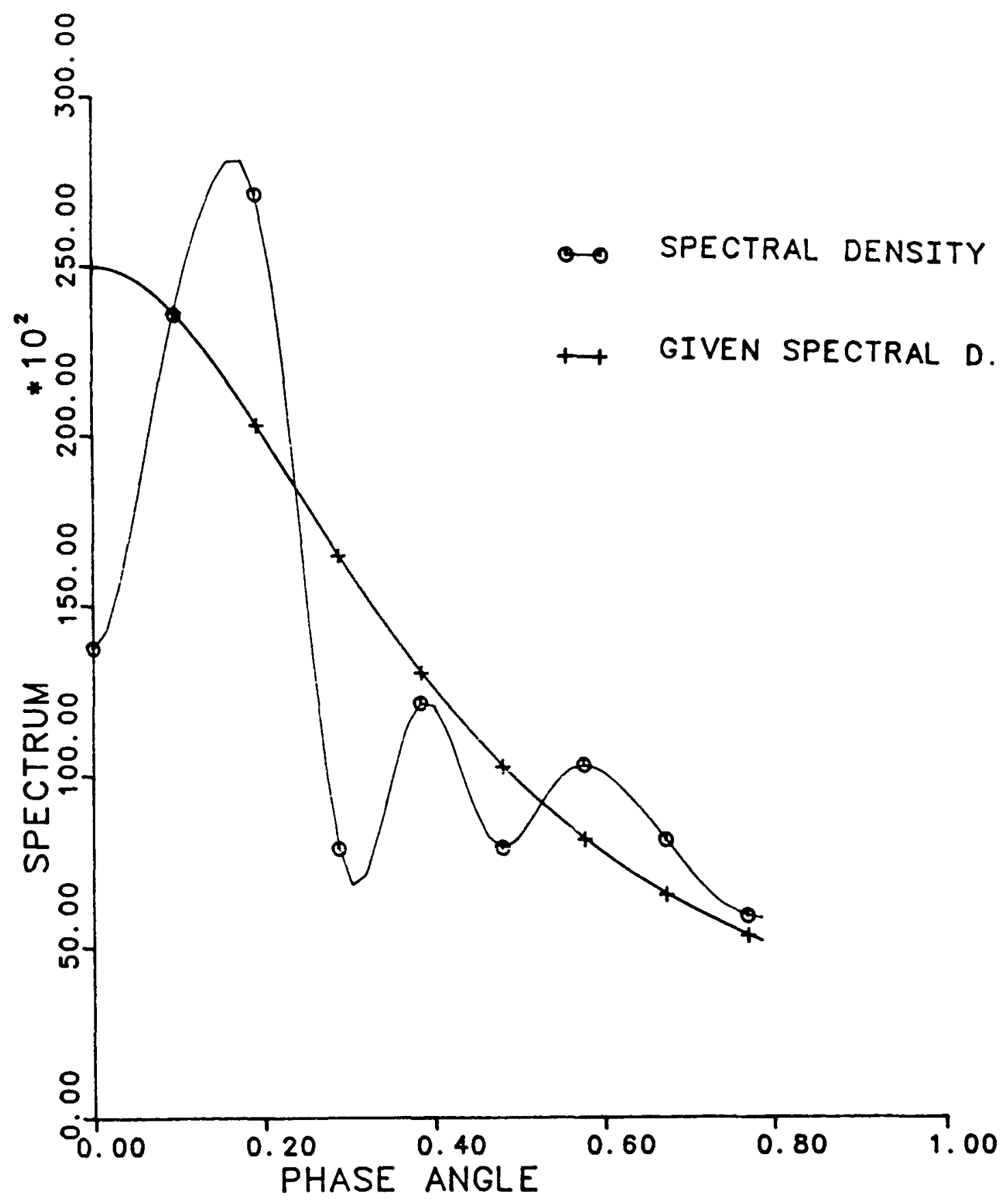

Figure V-2. Comparison of input and generated spectral density functions.

50X50, $\quad D X=D Y=2, \quad P=5, \quad C O V=1$ 


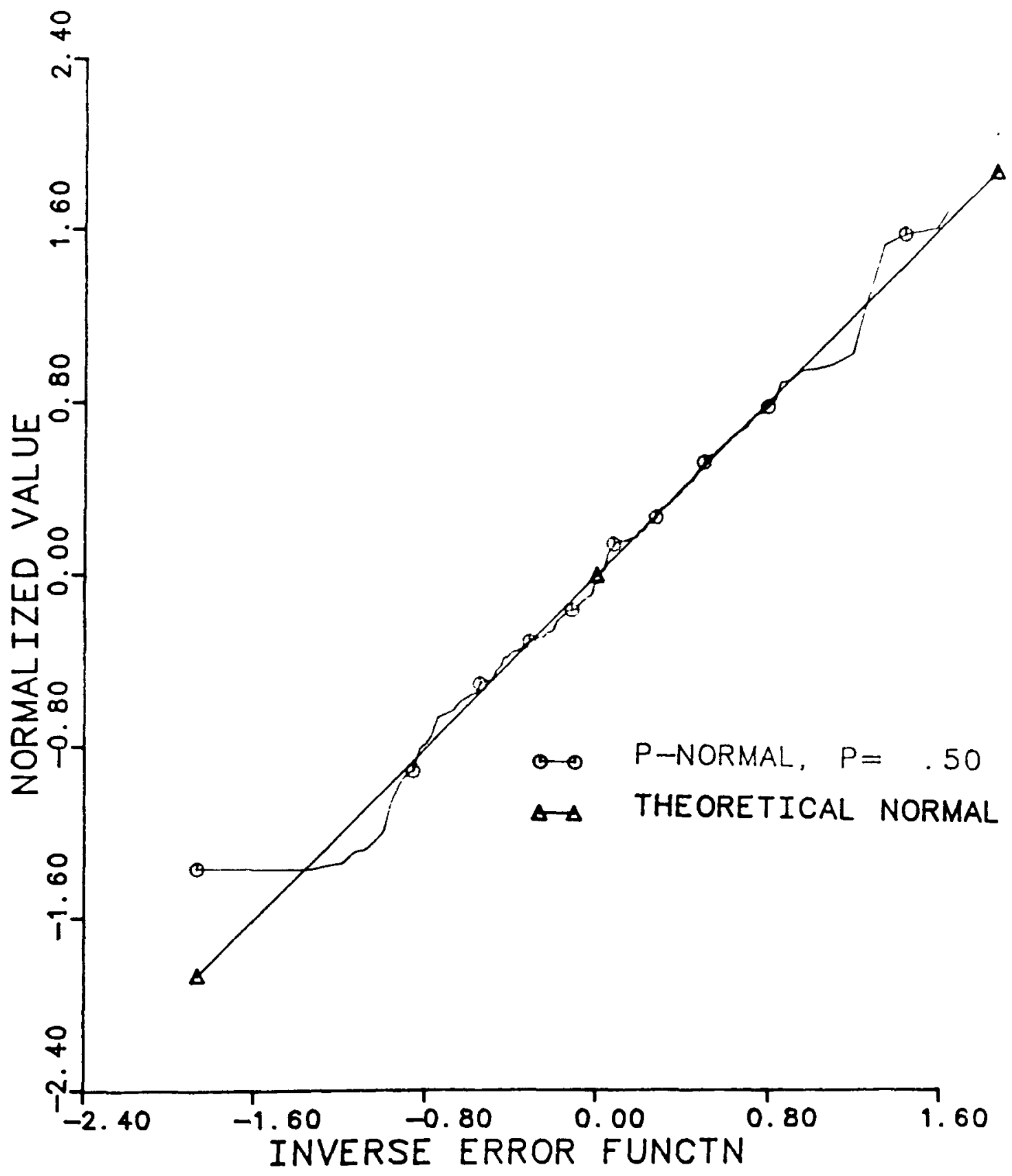

Figure V-3. Generated and input cumulative probability density functions. 


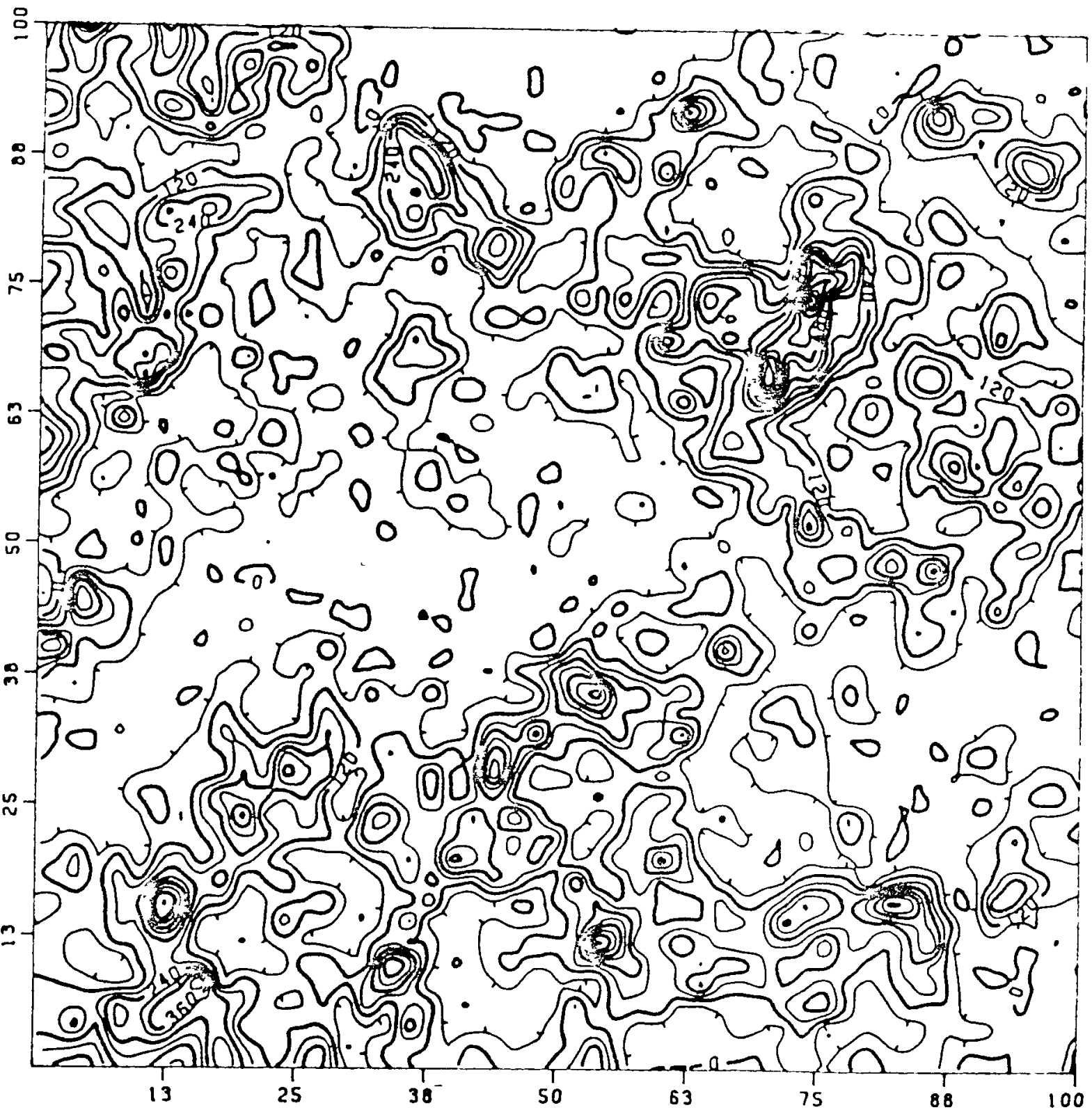

Figure V-4. Generated Contour Plots. 
PORE LEVEL MODELLING

The basic objective of this section is to investigate heterogeneity from a pore level approach, specifically, to investigate how pore-level heterogeneity is related to heterogeneity of bulk-scale petrophysical properties. Since all petrophysical flow properties depend upon pore network characteristics, this approach should lead to a better understanding of how stochastic distributions of various petrophysical properties are related.

The initial conceptual pore model we have chosen consists of a three-dimensional array of relatively large "pore bodies" inter-connected by smaller size "pore throats". Justification of this approach can be seen in pore casts and scanning-electron microscope photographs of pore structure (Swanson, 1979; Wunderlich, 1985). When we compare model parameters with actual reservoir permeable media, pore body sizes are controlled primarily by the grain-size distribution of the matrix. Pore throat sizes would be more a function of grain angularity and diagenetic effects.

Because of their relatively large size, pore bodies are assumed not to contribute to either flow transmissibility (permeability) or capillary pressures, and are modelled as equivalent spheres. Volumes occupied by pore bodies are based on stochastically distributed values for an equivalent pore body diameter, $d_{b}$. The volume of a pore body is then given by

$$
v_{b i}=\frac{\pi}{6} d_{b}^{3}
$$


For the initial part of the study we assume a structure whereby each pore body has six connecting pore throats in a three-dimensional cubic arrangement. Other structures and different levels of connectedness are possible and will evertually be considered. Pore throats are treated as capillary tubes, characterized by both a stochastically-distributed equivalent diameter, $d_{t}$, and an equivalent length, $I_{t}$. Pore-throat volumes are thus calculated as:

$$
v_{t i}=\frac{\pi}{4} d_{t}^{2} t
$$

Capillary pressure across a given pore-throat is then:

$$
\mathrm{P}_{\mathrm{c}}=\frac{4 \sigma \cos \theta}{\mathrm{d}_{\mathrm{t}}}
$$

where $\sigma$ is the interfacial tension and $\theta$ is the contact angle. Viscous pressure drops across throats are determined assuming a flow conductance, $(\mathrm{C}=\Delta \mathrm{P} / \mathrm{q})$, based on a Poiseuilles Law functional relationship:

$$
c=\frac{\pi d_{t}^{4}}{128 \mu I_{t}}
$$

Prior to initiating this study, a model had already been developed at the University of Texas for investigating capillary pressure-saturation curves in pore networks. Using stochastically-distributed pore-body radii, pore throat radii, and pore - throat lengths, the model calculated two-phase fluid distributions in pore networks by determining a non-wetting phase invasion sequence based on movement of fluid interfaces with minimum capillary pressures (largest pore-throat sizes). This model was 
successful in replicating actual capillary pressure measurements, but as expected, the results were not non-unique.

By only looking at capillary pressure phenomena, it was not necessary for the existing model to consider the relationship between pore network dimensions and bulk media dimensions. This is because capillary pressures only depend on the radii of pores, and saturations are a fraction of pore volume. Also, by considering further bulk petrophysical properties and constraining pore networks to geologically realistic ones, the uniqueness problem should diminish.

We are currently in the next phase of model development, which is to modify the existing model to generate values for porosity and permeability in addition to capillary pressure-saturation. Unlike capillary pressure curves, determination of both porosity and permeability depend upon relating pore-level dimensions to bulk dimensions. As a first simplified approach to this problem, we have chosen to define a parameter, $B$, which is the ratio of average effective pore or throat dimension to an orthogonal bulk dimension. $\beta$ is thus used to account for geometrical orientation effects as well as the relationship between effective and actual lengths. It can be thought of in the same manner as tortuosity, $\tau$, where $e=1 / \tau^{2}$ (Dullien, 1979). A pore body in a given direction can then be assigned an associated bulk length given by:

$$
1_{c}=\frac{1}{B}\left(\frac{1}{2}\left(1_{t 1}+1_{t 2}\right)+d_{b}\right)
$$

where the 1 and 2 refer to pore connecting in the desired direction. A mean equivalent length for a11 pore bodies in all directions is then: 


$$
\overline{1}_{c}=\frac{1}{6}\left(\overline{1}_{t}+\bar{d}_{b}\right)
$$

We can then calculate the porosity of the network as:

$$
=\left(\frac{\hat{c}}{\overline{1}_{t}+\bar{d}_{b}}\right)^{3}\left(\frac{\pi}{4} \bar{d}_{t}^{2} l_{t}+\frac{\pi}{6} \bar{d}_{b}^{3}\right)
$$

Permeability, $k$, can also be found by solving for flow rates and pressure drops in the pore system as a pipe network, utilizing the pore-throat conductances. If a differential pressure is applied in the $x$-direction, permeability is found hy solving for $q \mu / L P$ for the system and then calculating permeability by:

$$
k=\frac{q}{\Delta P} \frac{L_{x}}{L_{y} L_{z}}
$$

where $I_{x}$, $I_{y}$, and $I_{z}$ are the bulk media lengths in the $x, y$, and $z$-directions, respectively. Or, with Eq. (V-6) for bulk lengths:

$$
k=\left(\left.\frac{\varepsilon}{\overline{1}_{t}+\bar{d}_{b}} \frac{\left(q / n_{y} n_{z}\right) \mu}{\left(\Delta P / n_{x}\right)}\right|_{j b u l k}\right.
$$

where, $n_{x}, n_{y}$, and $n_{z}$ denote the number of pore bodies in the $y$, and $z$-directions, and $L_{x}=\overline{1}_{c} n_{x}$, etc. Note that the quantity in the brackets is invariant with network size (neglecting statistical effects), since $q / n_{y}{ }_{z}$ is the average flow rate per cross-sectional pore and $\Delta \mathrm{P} / \mathrm{n}_{\mathrm{x}}$ is the average pressure drop per pore.

With this model, then, we have the following parameter dependencies:

Permeability

$$
\begin{aligned}
& d_{t}, 1_{t}, d_{b}, B \\
& d_{b}, \beta \text { (major) } \\
& d_{t}, 1_{t} \text { (minor) }
\end{aligned}
$$$$
\text { Porosity }
$$ 


$\begin{array}{ll}\text { Capillary pressure } & \mathrm{d}_{\mathrm{t}} \\ \text { Saturation } & \mathrm{d}_{\mathrm{b}} \text { (major) } \\ & \mathrm{d}_{t}, I_{t} \text { (minor) }\end{array}$

In addition, permeability and capillary pressure versus saturation are dependent on the topological arrangement of the pore network. Porosity, however, is not. Pore-throat parameters primarily affect permeability and capillary pressure, while pore-body parameters are more related to porosity and saturations. With this approach, we have four parameters, three of which are stochastically-distributed. If we assume each of these can be adequately characterized by a three-parameter distribution, a total of nine parameters is required to model the system. Initially, we plan to utilize beta-type probability distributions for the various parameters, although other distributions will be explored. We will also initially assume no spatial correlation. This too can easily be investigated at a later date through the methods of Part III.

Once methods for determination of porosity and permeability are added to the model, the next step willi be to perform a large number of numerical experiments to explore the general characteristics and range of response of the model to different parameter values. The purpose of this phase of the study will be to primarily determine general relations between network parameters and the various bulk petrophysical properties. We will also wish to explore the empirical relationships between capillary pressure vs saturation curves, porosity, and permeability reported in the literature. For example, Leverett (1941) suggested that capillary pressure vs saturation data 
Erom similar depositional environments could be correlated by plotting à dimensioniess j-iunction:

$$
j=\frac{P_{c}}{\sigma \cos \theta} \sqrt{\frac{k}{\phi}}
$$

Álso, Purcell (1949), Thomeer (1960, 1983) and Swanson (1981) have susgested correlations for predicting permeability from capillary pressure and porosity data.

There are severai extensions of this work that can easily be made in iuture studies. Some or the most important petrophysical properties that the pore network model can be used to study are: a) two-phase relative permeabiities, b) electrical resistivities (either single or multi-phase), and c) macroscopic dispersion coefficients.

Conciucinis Remarks - Nearly ali of the work in the part $V$ is in a transitional stage. We nevertheless conclude that the stochastic Eeneration scheme can model wuch oi the complexity exhibited by the eolian outcrop. Our task, therefore, is to generate such fieids, extract statistics from them and compare to the statistics derived from the field data. This is perhaps the only direct way to evaluate the efficacy of the geweration scheme in generating realistic fields. 
NOMENCLATURE for PART V

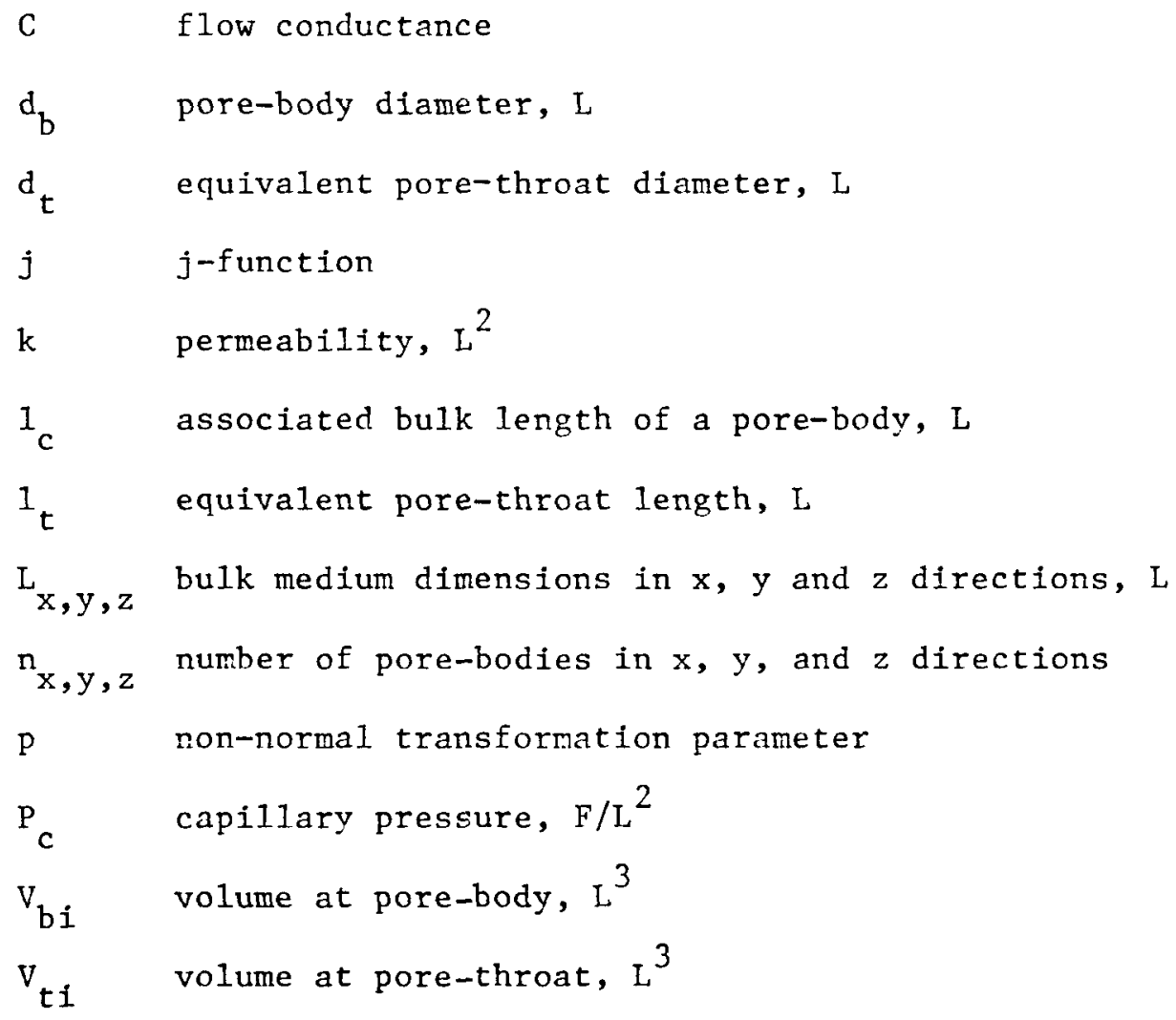




\section{PART V. REFERENCES}

Chandler, M.A. and Kocurek, G., "Depositional Controls on Permeability in an Eolian Sandstone Sequence, Page Sandstone, Northern Arizona," (in preparation) Bull. Am. Ass. Petrol. Geol., 1986.

Dullien, F. A. L. Porous Media, Fluid Transport and Pore Structure, Academic Press, New York (1979) 171.

Goggin, D. J., Chandler, M. A., Kocurek, G., and Lake, L. W., "Patterns of Permeability in Folian Deposits," SPE 14893, presented at the 1986 Society of Petroleum Engineers/Department of Energy 5th Symposium on Enhanced Oil Recover, Tulsa (April 20-23).

Leverett, M. C., "Capillary Pressure in Porous Solids," Trans., AIME (1941).

Montaglou, A., and Wilson, J. L., "The Turning Rands Method for Simulation of Random Fields Using Line Generation by a Spectral Method," Water Resources Research, Oct. 1982.

Purcell, W. R., "Capillary Pressures - Their Measurement Using Mercury and the Calculation of Permeability Therefrom," Trans., AIME (1949) $186,39-48$.

Swanson, B. F., "Visualizing Pore and Nonwetting Phase in Porous Rock," J. Pet. Tech. (Jan. 1979) 10-18.

Swanson, B. F., "A Simple Correlation Between Permeabilities and Mercury Capillary Pressure," J. Pet. Tech. (Dec. 1981) 2498-504.

Thomeer, J. H. M., "Introduction of a Pore Geometrical Factor Defined by the Capillary Pressure Curve," Trans., AIME (1960) 219, $354-58$.

Thomeer, J. H. M., "Air Permeability as a Function of Three Pore-Network Parameters," J. Pet. Tech. (Apr. 1983) 809-814.

Wunderlich, R. W., "Imaging of Wetting and Nonwetting Phase Distributions: Application to Centrifuge Capillary Pressure Measurements," SPE 14422, presented at SPE 60th Annual Technical Conference and Exhibition, Las Vegas, NV, Sept. 22-25, 1985. 